\title{
Chapter 3 \\ Structure-Activity Relationship Study \\ of PD 404182 Derivatives for the Highly \\ Potent Anti-HIV Agents
}

3,4-Dihydro-2H,6H-pyrimido[1,2-c][1,3]benzothiazin-6-imine (PD 404182) (1, Fig. 3.1) is a promising antiviral agent because of its high therapeutic index $\left(\mathrm{CC}_{50} /\right.$ $\mathrm{EC}_{50}>200$ ) and broad spectrum antiviral activities including against hepatitis $\mathrm{C}$ virus (HCV), simian immunodeficiency virus (SIV), and vesicular stomatitis virus (VSV), as well as HIV [1, 2]. In this chapter, the author describes the structureactivity relationship (SAR) studies of PD 404182 for the development of highly potent anti-HIV agents using the novel synthetic methods.

PD 404182 consists of three components, namely a 1,3-thiazin-2-imine core, and left-fused benzene and cyclic amidine moieties (Fig. 3.2). In order to obtain detailed insights into the relationships between compound structure and anti-HIV activity, the author planned to investigate substituent effects on each component: (I) derivatives with various heteroatom $(\mathrm{N}, \mathrm{S}$, and $\mathrm{O}$ ) arrangements on the 1,3thiazin-2-imine core; (II) pyrimido[1,2-c][1,3]thiazin-6-imine derivatives fused with a substituted benzene ring or a five- or six-membered aromatic heterocycle; and (III) benzo[ $e][1,3]$ thiazin-2-imine derivatives fused with a cyclic amidine ring with or without accessory alkyl or aryl groups.

The investigation began with the synthesis of tricyclic heterocycles with different combinations of heteroatoms on the 1,3-thiazin-2-imine core. As described in Chap. 2, the author developed synthetic methods for pyrimido[1,2-c][1,3]benzoxazine, pyrimido[1,2-c] quinazoline, and pyrimido[1,2-c][1,3]benzothiazine derivatives using $\mathrm{Cu}(\mathrm{II})$-mediated $\mathrm{C}-\mathrm{H}$ functionalization. This facilitates the introduction of oxygen, nitrogen, and sulfur functional groups at the ortho-position of 2-phenyl-1,4,5,6-tetrahydropyrimidine (2).

One-pot reaction for $\mathrm{Cu}(\mathrm{OAc})_{2}$-mediated $\mathrm{C}-\mathrm{H}$ functionalization of $\mathbf{2}$ and subsequent treatment with triphosgene provided a 1,3-oxazin-2-one derivative 4 (Scheme 3.1). The same one-pot procedure using thiophosgene produced a trace amount of the desired thiocarbonyl derivative 5. Treatment of the purified compound 3 with thiophosgene provided the desired 1,3-oxazin-2-thione 5 in high yield. 1,3-Oxazin-2-imine $\mathbf{6}$ was obtained by the reaction of $\mathbf{3}$ with $\mathrm{BrCN}$.

The $\mathrm{Cu}$-mediated $\mathrm{C}-\mathrm{N}$ bond formation of compound $\mathbf{2}$ with tert-butylcarbamate followed by spontaneous intramolecular cyclization afforded a pyrimido[1,2c]quinazolin-6-one scaffold 7 (Scheme 3.1). Subsequent treatment with 


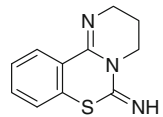

PD $404182(1)$

$\mathrm{EC}_{50}=0.44 \pm 0.08 \mu \mathrm{M}$

$\mathrm{CC}_{50}>100 \mu \mathrm{M}$

Fig. 3.1 Structure of PD 404182
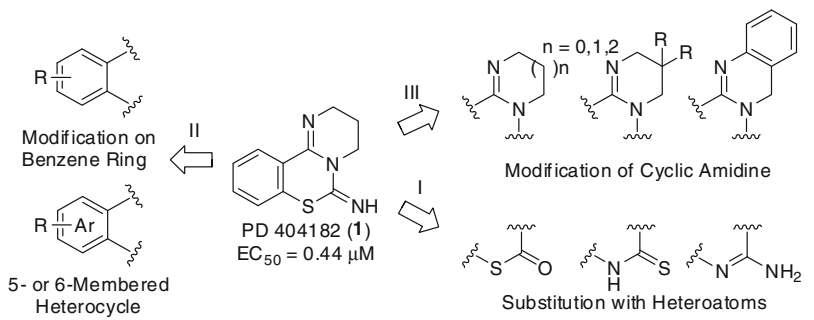

Fig. 3.2 Strategy for the SAR study of PD 404182

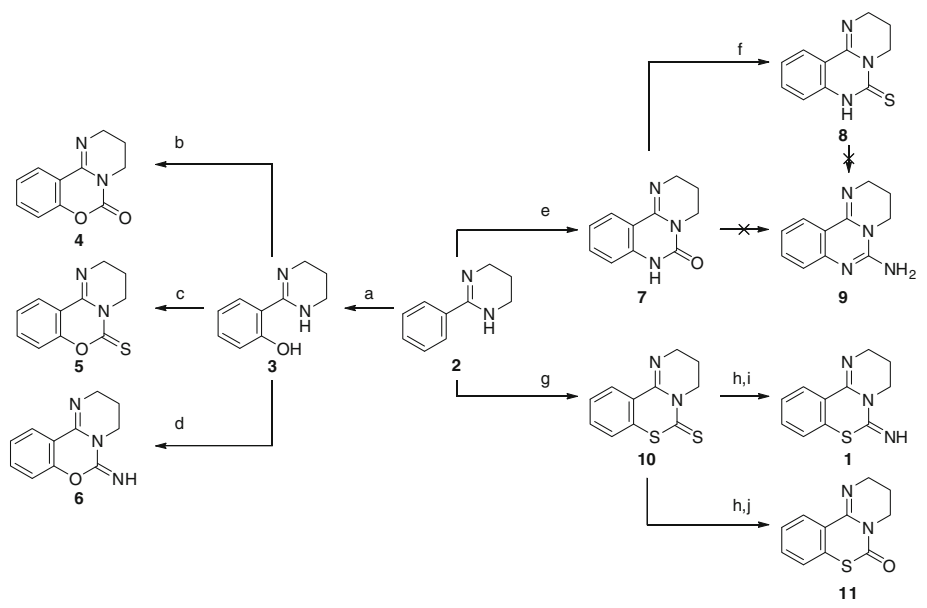

Scheme 3.1 Syntheses of various tricyclic heterocycles. Reagents and conditions (a) $\mathrm{Cu}(\mathrm{OAc})_{2}$, $\mathrm{H}_{2} \mathrm{O}, \mathrm{O}_{2}, \mathrm{DMF}, 130{ }^{\circ} \mathrm{C}, 69 \%$; (b) triphosgene, TMEDA, $\mathrm{CH}_{2} \mathrm{Cl}_{2}, 0{ }^{\circ} \mathrm{C}$ to rt, $70 \%$ [2 steps (a, b)]; (c) thiophosgene, $\mathrm{Et}_{3} \mathrm{~N}, \mathrm{CH}_{2} \mathrm{Cl}_{2}, 0{ }^{\circ} \mathrm{C}$ to $\mathrm{rt},>99 \%$; (d) $\mathrm{BrCN}, \mathrm{CH}_{2} \mathrm{Cl}_{2}, \mathrm{rt}, 34 \%$; (e) $\mathrm{Cu}(\mathrm{OAc})_{2}, \mathrm{BocNH}_{2}, \mathrm{O}_{2}, \mathrm{DMF}, 130{ }^{\circ} \mathrm{C}, 53 \%$; (f) Lawesson's reagent, xylene, reflux, $19 \%$; (g) $\mathrm{Cu}(\mathrm{OAc})_{2}, \mathrm{CS}_{2}, \mathrm{O}_{2}, 1,4$-dioxane, $130{ }^{\circ} \mathrm{C}, 11 \%$; (h) $\mathrm{NaOH}, \mathrm{MeOH}, \mathrm{H}_{2} \mathrm{O}$, reflux; (i) $\mathrm{BrCN}, \mathrm{EtOH}$, reflux, $61 \%$ [2 steps (h, i)]; (j) triphosgene, $\mathrm{Et}_{3} \mathrm{~N}, \mathrm{CH}_{2} \mathrm{Cl}_{2}, 0{ }^{\circ} \mathrm{C}$ to rt, $65 \%$ [2 steps (h, j)] 


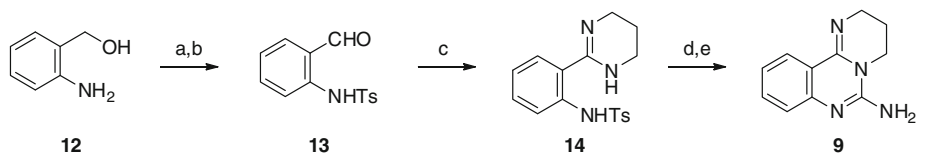

Scheme 3.2 Synthesis of 2-aminoquinazoline derivative 9. Reagents and conditions (a) $p$-TsCl, pyridine, $\mathrm{CHCl}_{3}$, rt; (b) PCC, silica gel, $\mathrm{CH}_{2} \mathrm{Cl}_{2}, \mathrm{rt}, 80 \%$ [2 steps (a, b)]; (c) 1,3-propanediamine, $\mathrm{I}_{2}, \mathrm{~K}_{2} \mathrm{CO}_{3}, t-\mathrm{BuOH}, 70{ }^{\circ} \mathrm{C}, 98 \%$; (d) conc. $\mathrm{H}_{2} \mathrm{SO}_{4}, 100{ }^{\circ} \mathrm{C}$, then $\mathrm{NaOH}, \mathrm{H}_{2} \mathrm{O}$; (e) $\mathrm{BrCN}, \mathrm{EtOH}$, reflux, $66 \%$ [2 steps $(\mathrm{d}, \mathrm{e})]$

Lawesson's reagent led to formation of the thiocarbonyl derivative $\mathbf{8}$. Since no hydrolysis of the carbonyl or thiocarbonyl group of compound $\mathbf{7}$ or $\mathbf{8}$ for construction of the 2-aminoquinazoline structure in $\mathbf{9}$ occurred, an alternative approach starting from 2-aminobenzyl alcohol $\mathbf{1 2}$ was used for the synthesis the 2-aminoquinazoline derivative 9 (Scheme 3.2). After protection and PCC oxidation of 12, oxidative amidination [3] provided 2-( $p$-tosylamino)phenyltetrahydropyrimidine (14). Deprotection followed by $\mathrm{BrCN}$-mediated cyclization of $\mathbf{1 4}$ provided the expected 2-aminoquinazoline derivative 9 .

To synthesize pyrimido[1,2-c][1,3]benzothiazine derivatives $\mathbf{1}$ and $\mathbf{1 1}$ (Scheme 3.1), compound 2 was exposed to $\mathrm{CS}_{2}$ in the presence of $\mathrm{Cu}(\mathrm{OAc})_{2}$ to directly afford a pyrimido[1,2-c][1,3]benzo-thiazine-6-thione scaffold 10. Hydrolysis of the thiocarbonyl group in $\mathbf{1 0}$ followed by treatment with $\mathrm{BrCN}$ or triphosgene provided 6-imino or 6-oxo derivatives ( 1 or 11), respectively.

Pyrimido[1,2-c][1,3]thiazin-6-imine derivatives 25-27 with a series of fused benzene and heterocycles were prepared by consecutive heterocumulene addition and $\mathrm{S}_{\mathrm{N}} \mathrm{Ar}$ reactions (Scheme 3.3). These reactions provide easy access to the construction of the 1,3-thiazin-2-imine derivatives more efficiently (Chap. 2.2) than the diversity-oriented $\mathrm{C}-\mathrm{H}$ functionalization approach (Chap. 2.1). The oxidative amidination of aromatic aldehydes 15-17 with an accessory functional group afforded the corresponding 2-phenyltetrahydropyrimidine derivatives 18-20. The pyrimido[1,2-c][1,3]thiazine-6-thione scaffold 21 was obtained by additions of 18f,g,i or 20s,t, $\mathbf{u}$ to $\mathrm{CS}_{2}$ followed by $\mathrm{S}_{\mathrm{N}} \mathrm{Ar}$-type $\mathrm{C}-\mathrm{S}$ bond formation. The desired 6-imino derivatives $\mathbf{2 5 f}, \mathbf{g}, \mathbf{i}$ and $\mathbf{2 7} \mathbf{s}, \mathbf{t}, \mathbf{u}$ were obtained via hydrolysis of the thiocarbonyl group of $\mathbf{2 1}$ followed by $\mathrm{BrCN}$ treatment. Alternatively, reactions of other 2-phenyltetrahydropyrimidines 18-20 with tert-butyl isothiocyanate afforded $N-(t-\mathrm{Bu})$-protected thiazinimine derivatives $\mathbf{2 2 - 2 4}$, which were treated with TFA to provide the expected products 25-27.

The intermediates $22 \mathbf{e}, \mathbf{2 2 k}$, and $23 \mathbf{k}$ were subjected to further manipulations to obtain the functionalized derivatives (Scheme 3.4). The nitro group of 22e was reduced by hydrogenation to form the 9-amino derivative $\mathbf{2 8}$. Alkylation of $\mathbf{2 8}$ afforded the 9-( $N$-methylamino) derivative 22b (eq 1). The 9-acetamide derivative 22c was obtained by treatment of $\mathbf{2 8}$ with acetic anhydride (eq 2). Sandmeyer reaction of $\mathbf{2 8}$ gave the 9 -azide derivative $22 \mathrm{p}$ (eq 3). $\mathrm{Me}_{2} \mathrm{~N}$ - and $\mathrm{MeO}$-substituted derivatives (22a, 23a, and 23f) were obtained by $\mathrm{Me}_{2} \mathrm{NH}$-mediated $\mathrm{N}$-arylation $[4,5]$ of the 9-bromo $22 \mathbf{k}$ and 10-bromo derivatives $\mathbf{2 3 k}$, and NaOMe-mediated 


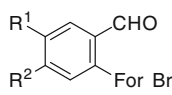

$15\left(R^{1}=H, R^{2}=\right.$ accessory group) $16\left(R^{1}=\right.$ accessory group, $\left.R^{2}=H\right)$

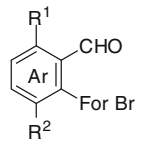

17
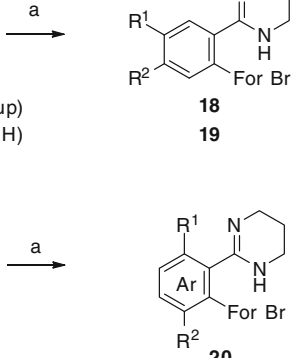

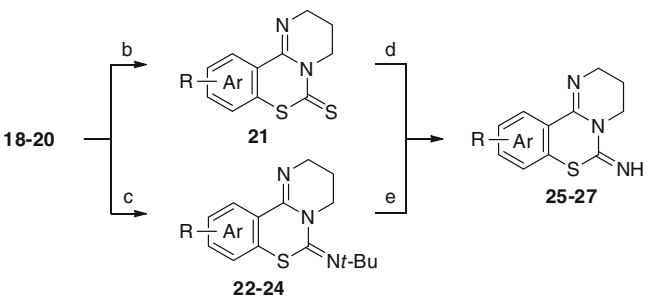

Scheme 3.3 Synthesis of pyrimido[1,2-c][1,3]thiazin-6-imine derivatives fused with substituted benzene and heterocycles (25-27). Reagents and conditions (a) 1,3-propanediamine, $\mathrm{I}_{2}, \mathrm{~K}_{2} \mathrm{CO}_{3}$, $t$ - $\mathrm{BuOH}, 70{ }^{\circ} \mathrm{C}, 58-91 \%$; (b) $\mathrm{NaH}, \mathrm{CS}_{2}, \mathrm{DMF}, 80{ }^{\circ} \mathrm{C}, 67$ to $>99 \%$; (c) $\mathrm{NaH}$ or $t$-BuOK, $t$-BuNCS, DMF or DMAc, rt-80 ${ }^{\circ} \mathrm{C}, 28-95 \%$; (d) (i) $\mathrm{NaOH}, \mathrm{MeOH}, \mathrm{H}_{2} \mathrm{O}$, reflux, (ii) $\mathrm{BrCN}$, EtOH, reflux, 32-68 \%; (e) TFA, MS4Å, $\mathrm{CHCl}_{3}$, reflux, 63-92\%

Ullmann coupling [6] of $\mathbf{2 3 k}$, respectively (eq 4 and 7). The 9-acetyl derivative 22d was obtained by Heck reaction [7] of $\mathbf{2 2 k}$ with 2-hydroxyethyl vinyl ether (eq 5). Other derivatives with a variety of functional groups $(\mathbf{2 2}, \mathbf{2 3}, \mathbf{2 9}$, and $\mathbf{3 0})$ were synthesized by Suzuki-Miyaura coupling reactions $[8,9]$ of $\mathbf{2 2 k}$ and $\mathbf{2 3 k}$ with boronic acids or their pinacol esters (eq 6 and 7). Final deprotection of the tert-butyl group in $\mathbf{2 2}, \mathbf{2 3}, \mathbf{2 9}$, and $\mathbf{3 0}$ afforded the 9- or 10-substituted pyrimido[1,2-c][1,3]benzothiazine derivatives $\mathbf{2 5}, \mathbf{2 6}, \mathbf{3 1}$, and 32, respectively.

Benzo[e][1,3]thiazine derivatives with various ring-sized and/or modified cyclic amidine moieties 36 were synthesized using standard synthetic methods (Scheme 3.5). Oxidative addition using a number of diamines $\mathbf{3 3}$ proceeded efficiently to form five- or six-membered rings $(\mathbf{3 4 a}-\mathbf{d})$. The same reaction for the seven-membered amidine (34e) was incomplete, but purification of the Boc-protected amidine $\mathbf{3 7}$ followed by subsequent deprotection of the Boc group gave the pure seven-membered amidine 34e. The resulting amidines were converted to cyclic-amidine-fused benzo[ $e][1,3]$ thiazin-2-imines 35 via tert-butyl isothiocyanate addition and an $\mathrm{S}_{\mathrm{N}} \mathrm{Ar}$ reaction. TFA-mediated deprotection gave the expected derivatives 36 . 
The synthesis of the spiropyrimidine-fused derivatives started with the dialkylation of malononitrile with dihaloalkanes $\left(\mathbf{3 8}, \mathbf{3 9}\right.$, or $\mathbf{4 1}$, Scheme 3.6). $\mathrm{BH}_{3}-$ mediated reduction of the alkylated malononitriles (42-44) followed by oxidative amidination with 4-bromo-2-fluorobenzaldehyde gave the 2-phenyl-1,4,5,6-tetrahydropyrimidine derivatives (45-47). Subsequent exposure of compounds $\mathbf{4 5 - 4 7}$ to tert-butylisothiocyanate provided the tetracyclic compounds $\mathbf{4 8 ,} \mathbf{5 0}$, and $\mathbf{5 2 a}$. Deprotection of the tert-butyl groups in compounds 48, 50, and 52a afforded the desired spiropyrimidine-fused benzothiazinimine derivatives $(\mathbf{4 9}, \mathbf{5 1}$, and 53a).

The substitution of the $p$-methoxybenzyl (PMB) group in compound 53a was also attempted (Scheme 3.6). The treatment of compound 52a with methyl chloroformate or acetyl chloride directly provided derivatives $\mathbf{5 2 b}$ and $\mathbf{5 2 c}$, respectively. A two-step procedure, including the removal of the PMB group by treatment with 1-chloroethyl chloroformate followed by modification with mesyl chloride $(\mathrm{MsCl})$ or trimethylsilyl isocyanate (TMSNCO) was used for the synthesis of the derivatives 52d and 52e, respectively, because the reaction of compound 52a with $\mathrm{MsCl}$ and TMSNCO failed. Deprotection of the tert-butyl group in 52b-e afforded the respective $N$-substituted derivatives 53b-e.

SARs of the central heterocyclic core in pyrimido[1,2-c][1,3]benzothiazines were carried out. Initially, the structural requirements of the 1,3-thiazin-2-imine core substructure in 1 (PD 404182) for anti-HIV activity were investigated (Table 3.1). The antiviral activities against the HIV-1 $1_{\text {IIIB }}$ strain were evaluated using the MAGI assay [10]. Substitution of the imino group in $\mathbf{1}$ with a carbonyl group (11) resulted in a significant decrease in anti-HIV activity $\left(\mathrm{EC}_{50}=8.94 \mu \mathrm{M}\right) . \quad$ Pyrimido[1,2-c][1,3]benzoxazines (4-6), pyrimido[1,2-c] quinazolines (7-9), and pyrimido[1,2-c] [1,3]benzothiazine-6-thione (10), in which the 1-sulfur and/or 2-imino groups in $\mathbf{1}$ were modified, showed no activity. These results suggested that both the 1-sulfur atom and the 2-imino group are indispensable functional groups for the inhibitory activity against HIV infection, and may be involved in potential interactions with the target molecules.

A series of derivatives with modification of the benzene substructure in the pyrimido[1,2-c][1,3]benzothiazine were evaluated for anti-HIV activity (Table 3.2). The addition of positively charged $N, N$-dimethylamino (25a) and $N$ methylamino groups (25b) at the 9-position significantly decreased the anti-HIV activity. The 9-acetamide group (25c), which has hydrogen bond donor/acceptor abilities, also attenuated the bioactivity. The acetyl (25d) and nitro (25e) groups, with hydrogen acceptor properties, induced slight decreases in the anti-HIV activity. In contrast, derivatives with less-polarized substituents (25f-o and 25q) at this position generally reproduced the potent anti-HIV activity of $\mathbf{1}$. In terms of the electron-donating or -withdrawing properties of the substituent groups on the benzene substructure, good correlations were not observed. For example, the electron-donating methoxy (25f), methyl (25g), and $n$-butyl groups $(\mathbf{2 5 h})$, and the electron-withdrawing fluoro (25i) and trifluoromethyl groups (25j) exhibited similar anti-HIV activities $\left(\mathrm{EC}_{50}=0.44-0.57 \mu \mathrm{M}\right)$, indicating that the antiviral activity is independent of the electronic state of the 1,3-benzothiazin-2-imine core in forming potential $\pi$-stacking interaction(s) with the target molecules. Among 


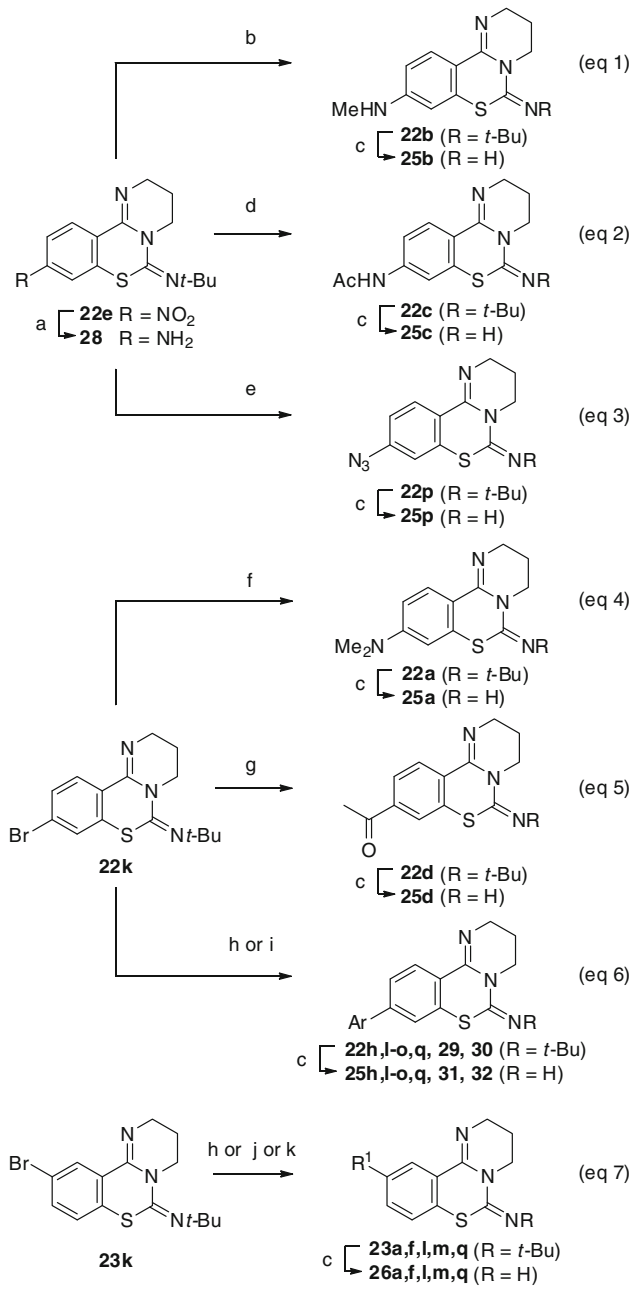

Scheme 3.4 Synthesis of 9- or 10-substituted pyrimido[1,2-c][1,3]benzothiazin-6-imine derivatives. Reagents and conditions (a) $\mathrm{H}_{2}, 10 \% \mathrm{Pd} / \mathrm{C}, \mathrm{EtOH}, \mathrm{rt}, 88 \%$; (b) $\mathrm{NaOMe},\left(\mathrm{CH}_{2} \mathrm{O}\right)_{n}$, $\mathrm{MeOH}$, reflux, then $\mathrm{NaBH}_{4}, 91 \%$; (c) TFA, MS4Å, $\mathrm{CHCl}_{3}$, reflux, 37-95\%; (d) $\mathrm{Ac}_{2} \mathrm{O}, \mathrm{DMAP}$, $\mathrm{Et}_{3} \mathrm{~N}, \mathrm{CH}_{2} \mathrm{Cl}_{2}, \mathrm{rt},>99 \%$; (e) $\mathrm{NaNO}_{2}, \mathrm{AcOH}, \mathrm{H}_{2} \mathrm{O}, 0{ }^{\circ} \mathrm{C}$, then $\mathrm{NaN}_{3}, 70 \%$; (f) $\mathrm{Pd}(\mathrm{OAc})_{2}, t-\mathrm{Bu}_{3} \mathrm{P}$, $\mathrm{NHMe}_{2}, \mathrm{THF}, \mathrm{KO} t-\mathrm{Bu}$, toluene, reflux, $>99 \%$; (g) 2-hydroxyethylvinylether, $\mathrm{Pd}(\mathrm{OAc})_{2}$, 1,3-bis(diphenylphosphino)propane, $\mathrm{K}_{2} \mathrm{CO}_{3}, \mathrm{H}_{2} \mathrm{O}, 90{ }^{\circ} \mathrm{C}, 13 \%$ [2 steps (g, c)]; (h) $\mathrm{R}-\mathrm{B}(\mathrm{OH})_{2}$ or R-Bpin, $\mathrm{Pd}\left(\mathrm{PPh}_{3}\right)_{4}, \mathrm{PdCl}_{2}$ (dppf) $\cdot \mathrm{CH}_{2} \mathrm{Cl}_{2}, \mathrm{~K}_{2} \mathrm{CO}_{3}$, toluene or 1,4-dioxane, $\mathrm{EtOH}, \mathrm{H}_{2} \mathrm{O}$, reflux, 62-96\%; (i) $n-\mathrm{BuB}(\mathrm{OH})_{2}, \mathrm{Pd}_{2}(\mathrm{dba})_{3}, \mathrm{P}(t-\mathrm{Bu})_{3}, \mathrm{CsCO}_{3}, 1,4$-dioxane, reflux, $6 \%$ (for 22h); (j) $\mathrm{Pd}\left(\mathrm{P} t-\mathrm{Bu}_{3}\right)_{2}, \mathrm{NHMe}_{2}, \mathrm{THF}, \mathrm{KO} t$-Bu, toluene, $170{ }^{\circ} \mathrm{C}, 67 \%$ (for 23a); (k) $\mathrm{CuBr}, \mathrm{NaOMe}$, $\mathrm{MeOH}, \mathrm{DMF}$, reflux, $40 \%$ (for $\mathbf{2 3 f}$ ) 
the hydrophobic substituents at this position, bromo (25k), phenyl (25l), vinyl $(\mathbf{2 5 m})$, styryl (25n), and pentenyl groups (25o) induced inhibitory activity two or three times greater than that of $1\left(\mathrm{EC}_{50}=0.18-0.25 \mu \mathrm{M}\right)$. Modification with photoreactive azido (25p) and benzoylphenyl groups (25q) maintained the inhibitory activity; these could be used as probe molecules to identify the target molecule(s) of 1 [11-13].

Similar SARs were observed for modification at the 10-position of pyrimido[1,2-c] [1,3]benzothiazine. Addition of positively charged $N, N$-dimethylamino (26a) and polarized nitro groups (26e) reduced the anti-HIV activity $\left(\mathrm{EC}_{50}=2.12\right.$ and $3.00 \mu \mathrm{M}$, respectively). Hydrophobic groups including methoxy (26f), methyl (26g), bromo (26k), phenyl (26l), vinyl (26m), and 4-benzoylphenyl (26q) had favorable effects on the bioactivity $\left(\mathrm{EC}_{50}=0.24-0.67 \mu \mathrm{M}\right)$, suggesting potential hydrophobic interactions of these additional functional groups with the target molecule(s).

Further miscellaneous modifications of benzothiazine substructure were also investigated (Table 3.2). The naphtho[2,3-e][1,3]thiazine derivative 27r, with a 9,10-fused benzene, exhibited anti-HIV activity equipotent to that of the parent 1 $\left(\mathrm{EC}_{50}=0.56 \mu \mathrm{M}\right)$. A 6 -fold decrease in the anti-HIV activity of the pyridinefused pyrido[3,2-e][1,3]thiazine derivative $(27 \mathrm{~s})$ was observed $\left(\mathrm{EC}_{50}=2.55 \mu \mathrm{M}\right)$. In addition, introduction of 8-bromo (27k) and 8,9-fused benzene (27t, naphtho[2,1-e][1,3]thiazine) substituents on benzothiazine resulted in a loss of activity, suggesting that modification at the 8-position was inappropriate for favorable interactions with the target molecule(s). The 11-fluoro derivative $27 \mathbf{i}$ and thiophene-fused 27u, the latter of which has 5-6-6 framework (thieno[2,3-e][1,3]thiazine), exhibited four times lower and no inhibitory potencies, respectively.

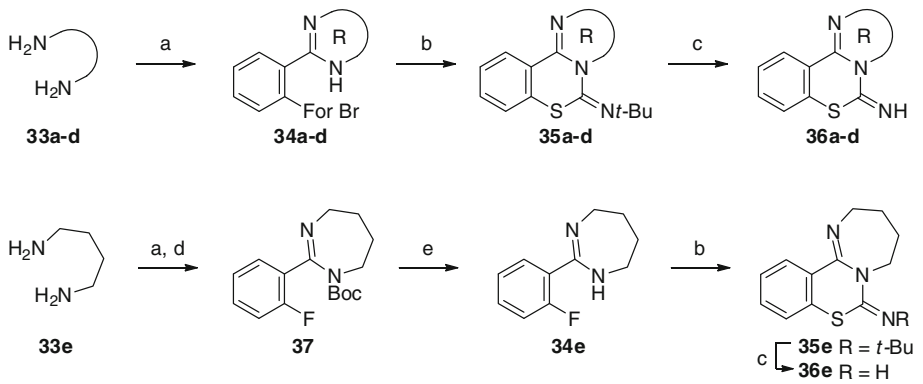

Scheme 3.5 Synthesis of benzo[ $[e][1,3]$ thiazine derivatives with fused cyclic amidines. Reagents and conditions (a) 2-fluorobenzaldehyde or 2-bromobenzaldehyde, $\mathrm{I}_{2}, \mathrm{~K}_{2} \mathrm{CO}_{3}, t$ - $\mathrm{BuOH}, 70{ }^{\circ} \mathrm{C}$, 68-79 \%; (b) NaH, $t$-BuNCS, DMF, rt $-80{ }^{\circ} \mathrm{C}, 18-50 \%$; (c) TFA, MS4 $\AA, \mathrm{CHCl}_{3}$, reflux, 16-86 \%; (d) $\mathrm{Boc}_{2} \mathrm{O}, \mathrm{Et}_{3} \mathrm{~N}$, DMAP, $\mathrm{CH}_{2} \mathrm{Cl}_{2}$, rt, $37 \%$ [2 steps (a, d)]; (e) TFA, $\mathrm{CHCl}_{3}$, reflux, $80 \%$ 


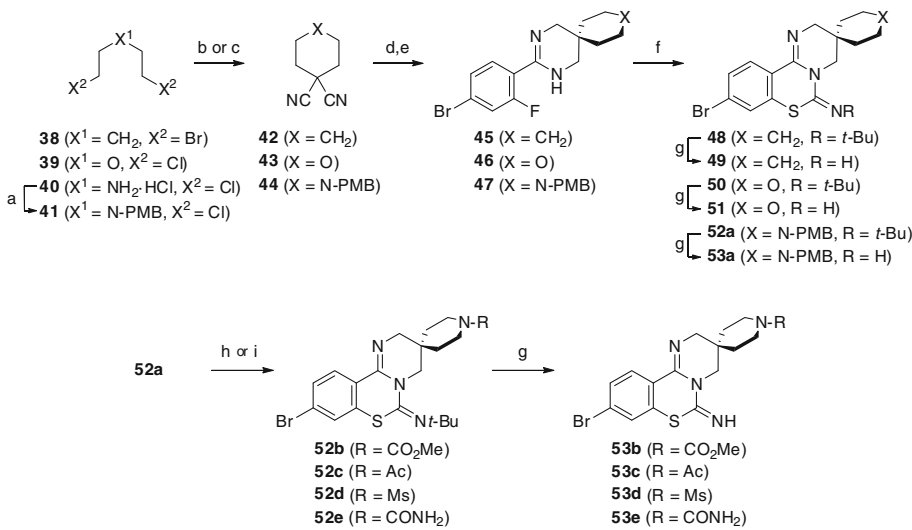

Scheme 3.6 Synthesis of spiropyrimidine-fused benzothiazinimine derivatives. Reagents and conditions (a) (i) 4-methoxybenzoyl chloride, $\mathrm{Et}_{3} \mathrm{~N}, \mathrm{CH}_{2} \mathrm{Cl}_{2}$, rt; (ii) $\mathrm{LiAlH}_{4}, \mathrm{Et}_{2} \mathrm{O}, \mathrm{rt}, 75 \%$ (2 steps); (b) malononitrile, DBU, DMF, 50 ${ }^{\circ} \mathrm{C}, 8-60 \%$ (for 42 and 43); (c) malononitrile, $\mathrm{K}_{2} \mathrm{CO}_{3}$, DMF, $65{ }^{\circ} \mathrm{C}, 85 \%$ (for 44); (d) $\mathrm{BH}_{3}$, THF, $0{ }^{\circ} \mathrm{C}$ to rt; (e) 4-bromo-2-fluorobenzaldehyde, $\mathrm{I}_{2}, \mathrm{~K}_{2} \mathrm{CO}_{3}$, t-BuOH, $70{ }^{\circ} \mathrm{C}, 11-62 \%$ [2 steps (d,e)]; (f) NaH, t-BuNCS DMF, rt $-80{ }^{\circ} \mathrm{C}$, 78-94 \%; (g) TFA, MS4Å, $\mathrm{CHCl}_{3}$, reflux, 65-94\%. (h) $\mathrm{ClCO}_{2} \mathrm{Me}$ or $\mathrm{AcCl}, \mathrm{CH}_{2} \mathrm{Cl}_{2}, 0{ }^{\circ} \mathrm{C}$, $81-96 \%$ (for 52b or 52c); (i) (i) 1-chloroethyl chloroformate, $\mathrm{Et}_{3} \mathrm{~N}, \mathrm{CH}_{2} \mathrm{Cl}_{2}, 0{ }^{\circ} \mathrm{C}$, then $\mathrm{MeOH}$, reflux, (ii) $\mathrm{MsCl}$ or TMSNCO, $\left(\mathrm{Et}_{3} \mathrm{~N}\right), \mathrm{CH}_{2} \mathrm{Cl}_{2}, \mathrm{rt}, 29-82 \%$ (2 steps, for 52d or 52e)

Table 3.1 SARs for 1,3-thiazin-2-imine core

\begin{tabular}{llll} 
& & \\
\hline Compound & $\mathrm{X}$ & $\mathrm{Y}$ & $\mathrm{EC}_{50}(\mu \mathrm{M})^{\mathrm{a}}$ \\
\hline $\mathbf{1}$ & $\mathrm{S}$ & $\mathrm{NH}$ & $0.44 \pm 0.08$ \\
$\mathbf{5}$ & $\mathrm{O}$ & $\mathrm{O}$ & $>10$ \\
$\mathbf{6}$ & $\mathrm{O}$ & $\mathrm{S}$ & $>10$ \\
$\mathbf{7}$ & $\mathrm{O}$ & $\mathrm{NH}$ & $>10$ \\
$\mathbf{8}$ & $\mathrm{NH}$ & $\mathrm{O}$ & $>10$ \\
$\mathbf{9}$ & $\mathrm{NH}$ & $\mathrm{S}$ & $>10$ \\
$\mathbf{1 0}$ & $\mathrm{NH}$ & $\mathrm{NH}$ & $>10$ \\
$\mathbf{1 1}$ & $\mathrm{S}$ & $\mathrm{S}$ & $8.94 \pm 1.07$ \\
\hline
\end{tabular}

${ }^{\mathrm{a}} \mathrm{EC}_{50}$ values represent the concentration of compound required to inhibit the HIV-1 infection by $50 \%$ and were obtained from three independent experiments

On the basis of the above SAR data for the benzene substructure in 1 (PD 404182), the author expected that introduction of a hydrophobic group at the pyrimido[1,2-c][1,3]benzothiazine 9-position would be the most promising. The next optimization to obtain more potent derivatives was therefore focused on 
Table 3.2 SARs for benzene part

\begin{tabular}{|c|c|c|c|c|}
\hline \multicolumn{2}{|c|}{ Compound } & \multirow[t]{2}{*}{$\mathrm{EC}_{50}(\mu \mathrm{M})^{\mathrm{a}}$} & \multirow{2}{*}{$\begin{array}{l}\text { Compound } \\
27 \mathbf{r}\end{array}$} & \multirow{2}{*}{$\frac{\mathrm{EC}_{50}(\mu \mathrm{M})^{\mathrm{a}}}{0.56 \pm 0.13}$} \\
\hline & & & & \\
\hline 1 & $\mathrm{R}=\mathrm{H}$ & $0.44 \pm 0.08$ & $27 \mathrm{~s}$ & $2.55 \pm 0.26$ \\
\hline $25 a$ & $\mathrm{R}=\mathrm{NMe}_{2}$ & $4.74 \pm 1.07$ & & \\
\hline $25 b$ & $\mathrm{R}=\mathrm{NHMe}$ & $>10$ & & \\
\hline $25 c$ & $\mathrm{R}=\mathrm{NHAc}$ & $>10$ & & \\
\hline $25 d$ & $\mathrm{R}=\mathrm{COMe}$ & $1.44 \pm 0.33$ & & \\
\hline $25 e$ & $\mathrm{R}=\mathrm{NO}_{2}$ & $1.13 \pm 0.18$ & & \\
\hline $25 f$ & $\mathrm{R}=\mathrm{OMe}$ & $0.57 \pm 0.09$ & $27 k$ & $>10$ \\
\hline $25 \mathrm{~g}$ & $\mathrm{R}=\mathrm{Me}$ & $0.49 \pm 0.10$ & & \\
\hline $25 \mathrm{~h}$ & $\mathrm{R}=\mathrm{n}$-butyl & $0.44 \pm 0.09$ & & \\
\hline $25 i$ & $\mathrm{R}=\mathrm{F}$ & $0.50 \pm 0.07$ & & \\
\hline $25 \mathbf{j}$ & $\mathrm{R}=\mathrm{CF}_{3}$ & $0.53 \pm 0.12$ & & \\
\hline $25 \mathrm{k}$ & $\mathrm{R}=\mathrm{Br}$ & $0.25 \pm 0.09$ & & \\
\hline 251 & $\mathrm{R}=\mathrm{Ph}$ & $0.24 \pm 0.04$ & & \\
\hline $25 \mathrm{~m}$ & $\mathrm{R}=$ vinyl & $0.18 \pm 0.05$ & $27 t$ & $>10$ \\
\hline $25 n$ & $\mathrm{R}=$ styryl & $0.25 \pm 0.05$ & & \\
\hline 250 & $\mathrm{R}=$ pentenyl & $0.24 \pm 0.11$ & & \\
\hline $25 p$ & $\mathrm{R}=\mathrm{N}_{3}$ & $0.43 \pm 0.06$ & & \\
\hline \multirow[t]{2}{*}{$25 q$} & $\mathrm{R}=\mathrm{C}_{6} \mathrm{H}_{4}(4-\mathrm{Bz})$ & $0.53 \pm 0.12$ & & \\
\hline & & & $27 \mathbf{i}$ & $1.68 \pm 0.19$ \\
\hline $26 a$ & $\mathrm{R}=\mathrm{NMe}_{2}$ & $2.12 \pm 0.26$ & $27 u$ & $>10$ \\
\hline $26 e$ & $\mathrm{R}=\mathrm{NO}_{2}$ & $3.00 \pm 0.59$ & & \\
\hline $26 f$ & $\mathrm{R}=\mathrm{OMe}$ & $0.53 \pm 0.04$ & & \\
\hline $26 \mathrm{~g}$ & $\mathrm{R}=\mathrm{Me}$ & $0.38 \pm 0.04$ & & \\
\hline $26 k$ & $\mathrm{R}=\mathrm{Br}$ & $0.24 \pm 0.05$ & & \\
\hline 261 & $\mathrm{R}=\mathrm{Ph}$ & $0.24 \pm 0.05$ & & \\
\hline $26 \mathrm{~m}$ & $\mathrm{R}=$ vinyl & $0.40 \pm 0.09$ & & \\
\hline $26 q$ & $\mathrm{R}=\mathrm{C}_{6} \mathrm{H}_{4}(4-\mathrm{Bz})$ & $0.67 \pm 0.16$ & & \\
\hline
\end{tabular}

${ }^{\mathrm{a}} \mathrm{EC}_{50}$ values represent the concentration of compound required to inhibit the HIV-1 infection by $50 \%$ and were obtained from three independent experiments

modification of the benzothiazine scaffold at position 9 with an additional aryl group (Tables 3.3, 3.4).

The author initially examined substituent effects at the para-position on the 9phenyl group of compound 25l. Modification with methoxycarbonyl (31a), cyano (31b), nitro (31c), and trifluoromethyl (31d) groups slightly reduced the anti-HIV activity $\left(\mathrm{EC}_{50}=0.44-0.81 \mu \mathrm{M}\right)$, whereas a significant decrease in the anti-HIV activity was observed for a carbamoyl group (31e), with hydrogen bond donor/ acceptor properties $\left(\mathrm{EC}_{50}=8.71 \mu \mathrm{M}\right)$. The hydrophobic methoxy (31f, 
Table 3.3 SARs for biphenyl-type derivatives

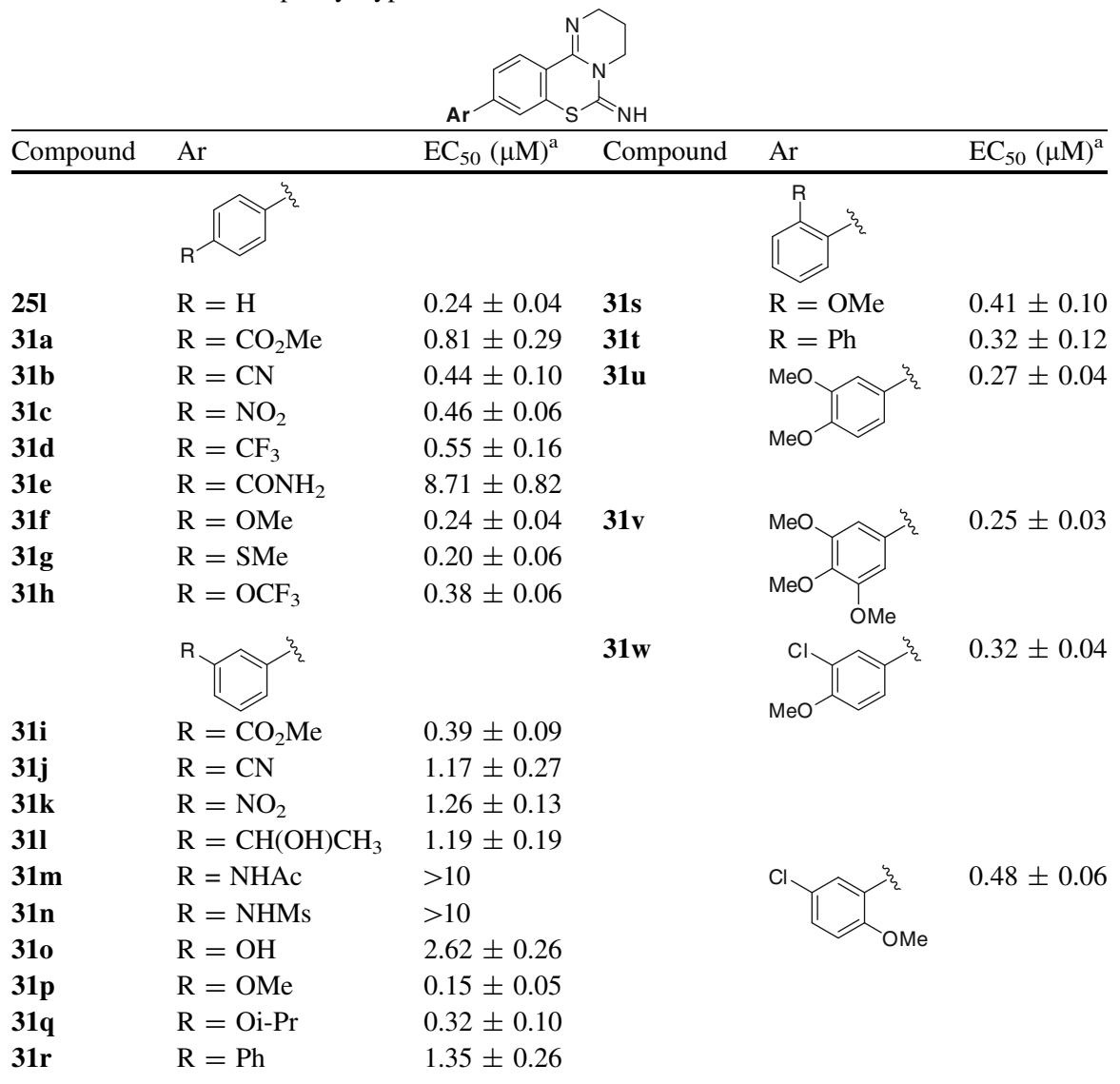

${ }^{\mathrm{a}} \mathrm{EC}_{50}$ values represent the concentration of compound required to inhibit the HIV-1 infection by $50 \%$ and were obtained from three independent experiments

$\left.\mathrm{EC}_{50}=0.24 \mu \mathrm{M}\right)$, methylthio $\left(\mathbf{3 1 g}, \mathrm{EC}_{50}=0.20 \mu \mathrm{M}\right)$, and trifluoromethoxy $\left(\mathbf{3 1 h}, \mathrm{EC}_{50}=0.38 \mu \mathrm{M}\right)$ groups had favorable effects on the anti-HIV activity.

Similar effects as a result of modification at the meta-position of the 9-phenyl group were observed. Addition of electron-withdrawing methoxycarbonyl (31i), cyano $(\mathbf{3 1 j})$, and nitro $(\mathbf{3 1 k})\left(\mathrm{EC}_{50}=0.39-1.26 \mu \mathrm{M}\right)$ groups resulted in slight decreases in anti-HIV activity. Hydrophilic (1-hydroxy)ethyl (311, $\left.\mathrm{EC}_{50}=1.19 \mu \mathrm{M}\right)$, acetylamino (31m), mesylamino (31n), and hydroxyl (31o, $\left.\mathrm{EC}_{50}=2.62 \mu \mathrm{M}\right)$ groups induced reduction or loss of anti-HIV activity. In contrast, a methoxy group (31p) improved the inhibitory activity $\left(\mathrm{EC}_{50}=0.15 \mu \mathrm{M}\right)$. The more hydrophobic isopropoxy group (31q) maintained the anti-HIV activity of $251\left(\mathrm{EC}_{50}=0.32 \mu \mathrm{M}\right)$, whereas a phenyl group (31r) decreased the inhibitory activity $\left(\mathrm{EC}_{50}=1.35 \mu \mathrm{M}\right)$. 
Table 3.4 SARs for biaryl-type derivatives

32f

${ }^{\mathrm{a}} \mathrm{EC}_{50}$ values represent the concentration of compound required to inhibit the HIV-1 infection by $50 \%$ and were obtained from three independent experiments

${ }^{\mathrm{b}}$ Cytotoxicity was observed at $10 \mu \mathrm{M}$

Similar anti-HIV activities of the ortho-methoxy (31s) and ortho-phenyl group (31t) to that of 251 were exhibited $\left(\mathrm{EC}_{50}=0.41\right.$ and $0.32 \mu \mathrm{M}$, respectively), suggesting that the twisted conformations of these 9-phenyl PD 404182 derivatives might not prevent the interaction with the target molecule(s).

In order to develop more potent anti-HIV agents, the author subsequently attempted bis and tris modifications of the 9-phenyl group in 25l. Modification with 9-(3,4-dimethoxy)phenyl $\left(\mathbf{3 1 u}, \quad \mathrm{EC}_{50}=0.27 \mu \mathrm{M}\right)$ or 9-(3,4,5-trimethoxy)phenyl $\left(31 \mathrm{v}, \mathrm{EC}_{50}=0.25 \mu \mathrm{M}\right)$ groups of the pyrimido[1,2-c][1,3]benzothiazine scaffold did not alter the bioactivity. Cl-modified derivatives 31w and 31x exhibited similar potencies $\left(\mathrm{EC}_{50}=0.32\right.$ and $0.48 \mu \mathrm{M}$, respectively).

Since the 9-(2-naphthyl)-modified analog (32a) exhibited slightly more potent anti-HIV activity $\left(\mathrm{EC}_{50}=0.20 \mu \mathrm{M}\right)$ compared with that of the 1-naphthyl congener $\left(\mathbf{3 2} \mathbf{b}, \mathrm{EC}_{50}=0.39 \mu \mathrm{M}\right.$, Table 3.4), the author further investigated modifications with a variety of 3,4-fused phenyl groups. Compound 32c with a 
1,3-dioxolane-fused phenyl group displayed activity twice as potent as that of compound $25 \mathrm{I}\left(\mathrm{EC}_{50}=0.15 \mu \mathrm{M}\right)$, whereas the 1,4-dioxane-fused derivative 32d and quinolin-6-yl derivative 32e exhibited less favorable effects $\left(\mathrm{EC}_{50}=0.26\right.$ and $0.25 \mu \mathrm{M}$, respectively). Introduction of an indolyl group (32f and $\mathbf{3 2 g}$ ) resulted in no anti-HIV activity and unexpected cytotoxicity.

Substitutions of the 9-phenyl group by various heterocyclic substructures were also investigated. Six-membered heterocycles such as pyridine (32h and 32i) slightly reduced the anti-HIV activity $\left(\mathrm{EC}_{50}=0.45\right.$ and $0.54 \mu \mathrm{M}$, respectively). The five-membered furan (32j), benzofuran (32k), thiophene (32l), benzothiophene (32m), and pyrazole (32n) derivatives maintained the original activity of $\mathbf{2 5 I}$ $\left(\mathrm{EC}_{50}=0.20-0.42 \mu \mathrm{M}\right)$. Notably, reduced anti-HIV activity was observed for the basic imidazole derivative $\left(\mathbf{3 2 o}, \mathrm{EC}_{50}=5.12 \mu \mathrm{M}\right)$.

A SAR study of the top-right cyclic amidine substructure was carried out. The five-membered dihydroimidazole derivative 36a had no anti-HIV activity (Table 3.5), suggesting that the five-membered ring may impair the critical interactions with the target molecule(s) via its small-sized ring strain or indirect effects on the thiazinimine core with a possibly altered conformation. Similarly, compound 36b with the phenyl-fused dihydropyrimidine substructure showed lower inhibitory activity $\left(\mathrm{EC}_{50}=3.78 \mu \mathrm{M}\right)$. Appending one or two methyl groups on the six-membered pyrimidine (36c and 36d) induced 1.5- to 2-fold higher inhibitory potencies $\left(\mathrm{EC}_{50}=0.35\right.$ and $0.24 \mu \mathrm{M}$, respectively) compared with that of the parent compound $\mathbf{1}$. In addition, compound 36e with a seven-membered tetrahydro-1,3-diazepine substructure exhibited similar anti-HIV activity to that of $1\left(\mathrm{EC}_{50}=0.31 \mu \mathrm{M}\right)$.

Above optimization studies indicated that the introduction of a hydrophobic group on the cyclic amidine substructures effectively improved the antiviral activity (compound 36c-e) by generating a potentially favorable interaction(s) with the target molecule(s). Therefore, anti-HIV activities of several spiropyrimidine fused derivatives were evaluated (Table 3.6). ${ }^{1}$ Cyclohexane (49) and $\mathrm{N}$ methoxycarbonylpiperidine (53b) derivatives exhibited the similar levels of antiHIV activity $\left(\mathrm{EC}_{50}=0.25\right.$ and $0.44 \mu \mathrm{M}$, respectively) to that of the dimethyl derivative 36d $\left(\mathrm{EC}_{50}=0.24 \mu \mathrm{M}\right)$. In contrast, the tetrahydropyran $(\mathbf{5 1})$ and $N-(p$ methoxybenzyl)piperidine (53a) derivatives exerted inhibitory activities that were 5-7-fold lower $\left(\mathrm{EC}_{50}=1.73\right.$ and $1.45 \mu \mathrm{M}$, respectively) than that of the parent dimethyl derivative 36d. The $N$-acetyl- (53c), $N$-methanesulfonyl- (53d), and $N$ carbamoyl- (53e) piperidine derivatives also provided reduced levels of antiviral activity $\left(\mathrm{EC}_{50}=1.81\right.$ to $\left.>10 \mu \mathrm{M}\right)$. With this in mind, the $N$-alkoxycarbonyl piperidine group was identified as a linkage for the introduction of additional functional group(s) to PD 404182 with potent anti-HIV activity (53b).

To investigate the mechanism of action of PD 404182 derivatives, a time of drug addition study was carried out (Fig. 3.3). In this experiment, the anti-HIV

\footnotetext{
${ }^{1}$ Because a 9-brominated derivative 25k exhibited more potent anti-HIV activity than compound 1 in the SAR study, the author employed compound $\mathbf{2 5 k}$ as a lead.
} 
Table 3.5 SARs for cyclic amidine core

36a

${ }^{\mathrm{a}} \mathrm{EC}_{50}$ values represent the concentration of compound required to inhibit the HIV-1 infection by $50 \%$ and were obtained from three independent experiments

Table 3.6 SARs for spiropyrimidine-fused derivatives

\begin{tabular}{lll} 
& $\mathrm{X}$ & \\
\hline Compound & $\mathrm{CH}_{2}$ & $0.25 \pm 0.01$ \\
$\mathbf{4 9}$ & $\mathrm{O}$ & $1.73 \pm 0.35$ \\
$\mathbf{5 3}$ & $\mathrm{N}-\mathrm{PMB}_{50}(\mu \mathrm{M})^{\mathbf{a}}$ \\
$\mathbf{5 3 b}$ & $\mathrm{N}-\mathrm{CO}_{2} \mathrm{Me}$ & $1.45 \pm 0.05$ \\
$\mathbf{5 3 c}$ & $\mathrm{N}-\mathrm{Ac}$ & $0.44 \pm 0.02$ \\
$\mathbf{5 3 d}$ & $\mathrm{N}-\mathrm{Ms}$ & $2.74 \pm 0.15$ \\
$\mathbf{5 3 e}$ & $\mathrm{N}-\mathrm{CONH} \mathrm{C}_{2}$ & $1.81 \pm 0.43$ \\
\hline
\end{tabular}

${ }^{\mathrm{a}} \mathrm{EC}_{50}$ values represent the concentration of compound required to inhibit the HIV-1 infection by $50 \%$ and were obtained from three independent experiments

activity profiles of $\mathbf{1}$ and its derivatives 32c were compared with those of wellknown anti-HIV agents such as an adsorption inhibitor (DS 5000) [14], fusion inhibitor (enfuvirtide) [15-17], NRTI (AZT) [18], NNRTI (nevirapine) [19], and integrase inhibitor (raltegravir) [20]. After inoculation of HeLa-CD4/CCR5-LTR/ $\beta$-gal cells with HIV-1 $1_{\text {IIIB }}$, each anti-HIV-1 drug was added at a $90 \%$ inhibitory effect concentration at the indicated time points. The inhibitory effects on the infection were determined by counting the blue cells $48 \mathrm{~h}$ later. This investigation revealed that compound 1 (PD 404182) had an inhibitory profile in the early stage 
Fig. 3.3 Time of drug addition profiles in the presence of Anti-HIV agents for infection of a HIV-1 $1_{\text {IIIB }}$ strain of HeLa-CD4/CCR5$\mathrm{LTR} / \beta$-gal cells

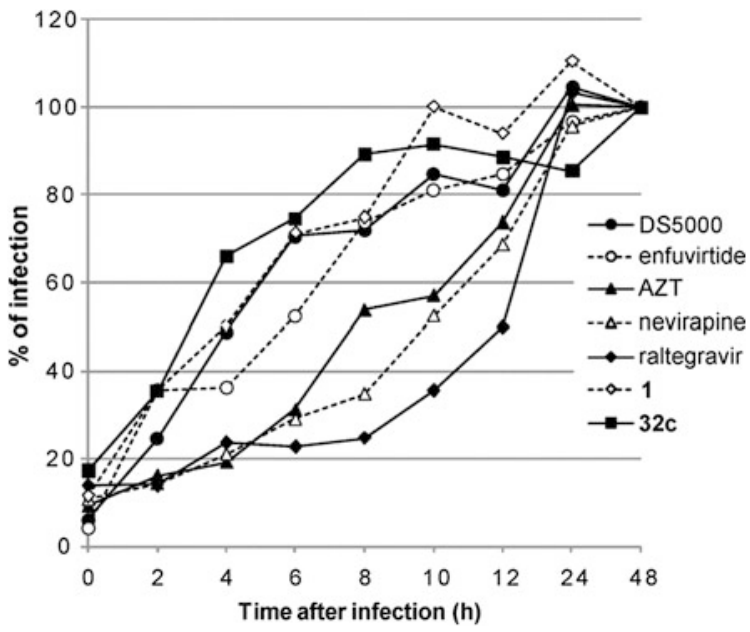

of viral infection similar to those of DS 5000 and enfuvirtide (Fig. 3.3). Identical profiles were observed for $\mathbf{1}$ and the most potent derivative 32c, indicating that the bioactivity profile is independent of the appended functional group(s).

To gain additional insights into the mechanism of action of PD 404182 derivatives, the antiviral activities against other HIV subtypes were evaluated (Table 3.7). Compound 1 was effective against not only HIV-1 $1_{\text {IIIB }}$ but also other two HIV-1 strains (HIV-1 $1_{\mathrm{NL} 4-3}$ and HIV-1 $1_{\mathrm{BaL}}$ ) with similar potency. Both HIV$1_{\text {IIIB }}$ and HIV-1 $1_{\text {NL4-3 }}$ strains utilize CXCR4 as a coreceptor for entry, while HIV$1_{\mathrm{BaL}}$ strain does CCR5, indicating that chemokine receptors CXCR4 and CCR5 are not the molecular targets of PD 404182 derivatives. The similar level of antiviral activity of 1 against HIV-2 (HIV-2 $2_{\text {EHO }}$ and HIV-2 $2_{\text {ROD }}$ ), which is mainly distributed in West Africa, was observed. Highly potent inhibitory activities of derivatives 32c and $\mathbf{3 6} \mathbf{d}^{2}$ against these HIV strains were observed, as in the case of the SAR study of the HIV-1 $1_{\text {IIIB }}$ strain discussed above. It has been well-known that NNRTIs are not effective against HIV-2, highlighting that PD 404182 derivatives do not act as NNRTIs. Although PD 404182 derivatives and enfuvirtide showed similar anti-HIV-1 profile in the time of drug addition assay, HIV-2 ${ }_{\mathrm{EHO}}$ and HIV$2_{\text {ROD }}$ infection were affected by PD 404182 derivatives, in contrast with the less effective enfuvirtide [21], suggesting that PD 404182 derivatives may not be directed at the HIV gp41 envelope protein. Recent reports have suggested that the antiviral activities of compound $\mathbf{1}$ against HIV, HCV, and pseudotype lentiviruses were derived from disruption of the structural integrities of virions [2]. Although the mechanism of action of PD 404182 derivatives is not fully understood at this

\footnotetext{
2 The cytotoxicity of compounds $\mathbf{1}, \mathbf{3 2 c}$ and $\mathbf{3 6 d}$ was not observed at $10 \mu \mathrm{M}$ in the MAGI assay. Further toxicity studies such as hemolytic activity or renal/liver accumulation may be needed to take a drug for long periods of time.
} 
Table 3.7 Anti-HIV activity of compounds $\mathbf{1}$, 32c, and 36d against other HIV strains

\begin{tabular}{|c|c|c|c|}
\hline \multirow[t]{2}{*}{ Strains } & \multicolumn{3}{|l|}{$\mathrm{EC}_{50}(\mu \mathrm{M})^{\mathrm{a}}$} \\
\hline & 1 & $32 \mathrm{c}$ & 36d \\
\hline HIV-1 $1_{\text {NL4-3 }}$ & $0.38 \pm 0.06$ & $0.25 \pm 0.03$ & $0.23 \pm 0.09$ \\
\hline $\mathrm{HIV}-1_{\mathrm{BaL}}$ & $0.37 \pm 0.06$ & $0.16 \pm 0.02$ & $0.13 \pm 0.05$ \\
\hline HIV-2 ${ }_{\text {ЕHO }}$ & $0.31 \pm 0.06$ & $0.17 \pm 0.03$ & $0.14 \pm 0.02$ \\
\hline HIV-2 $2_{\text {ROD }}$ & $0.30 \pm 0.06$ & $0.11 \pm 0.03$ & $0.10 \pm 0.04$ \\
\hline
\end{tabular}

${ }^{\mathrm{a}} \mathrm{EC}_{50}$ values represent the concentration of compound required to inhibit the HIV-1 infection by $50 \%$ and were obtained from three independent experiments

stage, the unidentified biomolecule(s) in viruses or host cells could be promising molecular targets for this new class of anti-HIV agents.

In conclusion, the author have designed and synthesized PD 404182 derivatives for a novel series of anti-HIV agents. Comprehensive SAR studies demonstrated that the 6-6-6 fused pyrimido[1,2-c][1,3]benzothiazine scaffold and the heteroatom arrangement in the thiazinimine moiety are indispensable for the inhibitory activity of 1 (PD 404182) against HIV infection. Optimization studies of the benzene and cyclic amidine rings indicate that the introduction of a hydrophobic group on the benzene ring is more effective in improving the antiviral activity, giving potential favorable interaction(s) with the target molecule(s). The most potent compound, 32c, had anti-HIV activity three times higher than that of the parent 1. In addition, PD 404182 derivatives could be promising agents for treatment of HIV-2 infection. The author also revealed, using a time of drug addition experiment, that PD 404182 derivatives prevent the HIV infection process at a fusion or binding process.

\subsection{Experimental Section}

\subsubsection{General Methods}

All moisture-sensitive reactions were performed using syringe-septum cap techniques under an Ar atmosphere and all glasswares were dried in an oven at $80{ }^{\circ} \mathrm{C}$ for $2 \mathrm{~h}$ prior to use. Melting points were measured by a hot stage melting point apparatus (uncorrected). For flash chromatography, Wakogel C-300E (Wako) or aluminum oxide 90 standardized (Merck) was employed. For preparative TLC, TLC silica gel 60 F254 (Merck) or TLC aluminum oxide 60 F254 basic (Merck), or $\mathrm{NH}_{2}$ Silica Gel 60 F254 Plate (Wako) were employed. For analytical HPLC, a COSMOSIL 5C18-ARII column $(4.6 \times 250 \mathrm{~mm}$, Nacalai Tesque, Inc., Kyoto, Japan) was employed with method $\mathrm{A}$ [a linear gradient of $\mathrm{CH}_{3} \mathrm{CN}$ containing $0.1 \%(\mathrm{v} / \mathrm{v})$ TFA] or method B [a linear gradient of $\mathrm{CH}_{3} \mathrm{CN}$ containing $0.1 \%(\mathrm{v} / \mathrm{v})$ $\mathrm{NH}_{3}$ ] at a flow rate of $1 \mathrm{~mL} / \mathrm{min}$ on a Shimadzu LC-10ADvp (Shimadzu Corp., Ltd., Kyoto, Japan), and eluting products were detected by UV at $254 \mathrm{~nm}$. 
Preparative HPLC was performed using a COSMOSIL 5C18-ARII column $(20 \times 250 \mathrm{~mm}$, Nacalai Tesque Inc. $)$ with a linear gradient of MeCN containing $0.1 \%$ (v/v) $\mathrm{NH}_{3}$ at a flow rate of $8 \mathrm{~mL} / \mathrm{min}$ on Shimadzu LC-6AD (Shimadzu corporation, Ltd). ${ }^{1} \mathrm{H}-\mathrm{NMR}$ spectra were recorded using a JEOL AL-400 or a JEOL ECA-500 spectrometer, and chemical shifts are reported in $\delta$ (ppm) relative to $\mathrm{Me}_{4} \mathrm{Si}\left(\mathrm{CDCl}_{3}\right)$ or DMSO (DMSO- $\left.d_{6}\right)$ as internal standards. ${ }^{13} \mathrm{C}-\mathrm{NMR}$ spectra were recorded using a JEOL AL-400 or JEOL ECA-500 spectrometer and referenced to the residual solvent signal. ${ }^{19} \mathrm{~F}-\mathrm{NMR}$ spectra were recorded using a JEOL ECA-500 and referenced to the internal $\mathrm{CFCl}_{3}\left(\delta_{\mathrm{F}} 0.00 \mathrm{ppm}\right) .{ }^{1} \mathrm{H}-\mathrm{NMR}$ spectra are tabulated as follows: chemical shift, multiplicity $(\mathrm{b}=$ broad, $\mathrm{s}=$ singlet, $\mathrm{d}=$ doublet, $\mathrm{t}=$ triplet, $\mathrm{q}=$ quartet, $\mathrm{m}=$ multiplet), coupling constant(s), and number of protons. Exact mass (HRMS) spectra were recorded on a JMS-HX/HX 110A mass spectrometer. Infrared (IR) spectra were obtained on a JASCO FT/IR4100 FT-IR spectrometer with JASCO ATR PRO410-S. The purity of the compounds was determined by combustion analysis or HPLC analysis as $>95 \%$ unless otherwise stated. Synthesis and characterization data of compounds 2, 4, 7, and $\mathbf{1 0}$ are shown in Chap. 2. Synthesis and characterization data of compounds 1, 18e-g, 18i, 19e, 20s, 20t, 21f, 21g, 21i, 21s, 21t, 34a and 35a are shown in Chap. 2.

\subsubsection{Synthesis of 3,4-Dihydro-2H,6H-pyrimido [1,2-c][1,3]benzoxazine-6-thione (5)}

2-(2-Hydroxyphenyl)tetrahydropyrimidine (3). DMF $(0.83 \mathrm{~mL})$ and water $(4.5 \mu \mathrm{L}, 0.25 \mathrm{mmol})$ were added to a flask 2-phenyl-1,4,5,6-tetrahydropyrimidine 2 (40.1 mg, $0.25 \mathrm{mmol})$ and $\mathrm{Cu}(\mathrm{OAc})_{2}(45.4 \mathrm{mg}, 0.25 \mathrm{mmol})$ under an $\mathrm{O}_{2}$ atmosphere. After being stirred at $130{ }^{\circ} \mathrm{C}$ for $20 \mathrm{~min}$, mixture was concentrated. The residue was purified by flash chromatography over aluminum oxide with $\mathrm{CHCl}_{3}-\mathrm{MeOH}(95: 5)$ to give the title compound $\mathbf{3}$ as brown solid (30.3 $\mathrm{mg}$, $69 \%$ ): IR (neat) $\mathrm{cm}^{-1}$ : 3257-3041 (OH), $1613(\mathrm{C}=\mathrm{N}) ;{ }^{1} \mathrm{H}-\mathrm{NMR}(500 \mathrm{MHz}$, DMSO- $\left.d_{6}\right) \delta: 1.84-1.88\left(2 \mathrm{H}, \mathrm{m}, \mathrm{CH}_{2}\right), 3.40\left(4 \mathrm{H}, \mathrm{t}, J=5.7 \mathrm{~Hz}, 2 \times \mathrm{CH}_{2}\right)$, 6.27-6.30 (1H, m, Ar), $6.47(1 \mathrm{H}, \mathrm{d}, J=8.6 \mathrm{~Hz}, \mathrm{Ar}), 7.04-7.08(1 \mathrm{H}, \mathrm{m}, \mathrm{Ar}), 7.45$ $(1 \mathrm{H}, \mathrm{dd}, J=8.0,1.7 \mathrm{~Hz}, \mathrm{Ar}), 12.09(1 \mathrm{H}, \mathrm{br} \mathrm{s}) ;{ }^{13} \mathrm{C}-\mathrm{NMR}\left(125 \mathrm{MHz}, \mathrm{CD}_{3} \mathrm{OD}\right) \delta$ : 20.0, 39.3 (2C), 111.2, 114.5, 124.4, 126.3, 135.1, 161.0, 172.4; MS (FAB) $\mathrm{m} / z$ (\%): $177\left(\mathrm{MH}^{+}, 100\right)$.

Compound 5. To a suspension of $3(33.0 \mathrm{mg}, 0.19 \mathrm{mmol})$ and $\mathrm{Et}_{3} \mathrm{~N}$ $(0.068 \mathrm{~mL}, 0.47 \mathrm{mmol})$ in $\mathrm{CH}_{2} \mathrm{Cl}_{2}(10.0 \mathrm{~mL})$ was added dropwise a solution of thiophosgene $(0.016 \mathrm{~mL}, 0.21 \mathrm{mmol})$ in $\mathrm{CH}_{2} \mathrm{Cl}_{2}(1.0 \mathrm{~mL})$ at $0{ }^{\circ} \mathrm{C}$. After being stirred at $\mathrm{rt}$ for $1 \mathrm{~h}$, the mixture was quenched with sat. $\mathrm{NaHCO}_{3}$. The whole was extracted with EtOAc. The extract was washed with sat. $\mathrm{NaHCO}_{3}$, brine, and dried over $\mathrm{MgSO}_{4}$. After concentration, the residue was purified by flash chromatography over silica gel with $n$-hexane-EtOAc (3:1) to give the title compound $\mathbf{5}$ as yellow solid (41.9 mg, >99\%): $\mathrm{mp} 135-136{ }^{\circ} \mathrm{C}$ (from $\mathrm{CHCl}_{3}-n$-hexane); IR 
(neat) $\mathrm{cm}^{-1}: 1655(\mathrm{C}=\mathrm{N}) ;{ }^{1} \mathrm{H}-\mathrm{NMR}\left(400 \mathrm{MHz}, \mathrm{CDCl}_{3}\right) \delta: 2.03-2.08(2 \mathrm{H}, \mathrm{m}$, $\left.\mathrm{CH}_{2}\right), 3.68\left(2 \mathrm{H}, \mathrm{t}, J=5.5 \mathrm{~Hz}, \mathrm{CH}_{2}\right), 4.30\left(2 \mathrm{H}, \mathrm{t}, J=6.1 \mathrm{~Hz}, \mathrm{CH}_{2}\right), 7.21(1 \mathrm{H}, \mathrm{d}$, $J=8.5 \mathrm{~Hz}, \mathrm{Ar}), 7.25-7.29(1 \mathrm{H}, \mathrm{m}, \mathrm{Ar}), 7.50-7.52(1 \mathrm{H}, \mathrm{m}, \mathrm{Ar}), 8.00(1 \mathrm{H}, \mathrm{d}$, $J=7.8 \mathrm{~Hz}, \mathrm{Ar}) ;{ }^{13} \mathrm{C}-\mathrm{NMR}\left(100 \mathrm{MHz}, \mathrm{CDCl}_{3}\right) \delta: 21.1,44.5,49.2,115.9,116.9$, 125.4, 125.7, 133.0, 139.7, 150.9, 180.8; Anal. calcd for $\mathrm{C}_{11} \mathrm{H}_{10} \mathrm{~N}_{2} \mathrm{OS}: \mathrm{C}, 60.53 ; \mathrm{H}$, 4.62; N, 12.83. Found: C, 60.23; H, 4.72; N, 12.62 .

\subsubsection{Synthesis of 3,4-Dihydro-2H,6H-pyrimido [1,2-c][1,3]benzoxazin-6-imine (6)}

2-(2-Hydroxyphenyl)tetrahydropyrimidine $3(5.4 \mathrm{mg}, 0.03 \mathrm{mmol})$ was suspended with $\mathrm{CH}_{2} \mathrm{Cl}_{2}(0.3 \mathrm{~mL})$ and added the solution of $\mathrm{BrCN}(3.3 \mathrm{mg}, 0.06 \mathrm{mmol})$ in $\mathrm{CH}_{2} \mathrm{Cl}_{2}(0.3 \mathrm{~mL})$. After being stirred for $1 \mathrm{~h}$ at $\mathrm{rt}$, the additional portion of $\mathrm{BrCN}$ (3.3 mg, $0.06 \mathrm{mmol})$ in $\mathrm{CH}_{2} \mathrm{Cl}_{2}(0.3 \mathrm{~mL})$ was added. After being stirred for $1 \mathrm{~h}$ at $\mathrm{rt}$, the mixture was concentrated. The residue was purified by preparative TLC over $\mathrm{NH}_{2}$ silica gel with $n$-hexane-EtOAc (1:1) to give the title compound $\mathbf{6}$ as colorless solid ( $2.1 \mathrm{mg}, 34 \%)$ : $\mathrm{mp} 104-105{ }^{\circ} \mathrm{C}$ (from $\mathrm{CHCl}_{3}-n$-hexane); IR (neat) $\mathrm{cm}^{-1}: 1639(\mathrm{C}=\mathrm{N}), 1611(\mathrm{C}=\mathrm{N}) ;{ }^{1} \mathrm{H}-\mathrm{NMR}\left(500 \mathrm{MHz}, \mathrm{CDCl}_{3}\right) \delta: 1.98-2.03(2 \mathrm{H}$, $\left.\mathrm{m}, \mathrm{CH}_{2}\right), 3.64\left(2 \mathrm{H}, \mathrm{t}, J=5.4 \mathrm{~Hz}, \mathrm{CH}_{2}\right), 3.93\left(2 \mathrm{H}, \mathrm{t}, J=6.0 \mathrm{~Hz}, \mathrm{CH}_{2}\right), 5.83(1 \mathrm{H}$, br s, NH), $6.99(1 \mathrm{H}, \mathrm{d}, J=8.0 \mathrm{~Hz}, \mathrm{Ar}), 7.15(1 \mathrm{H}, \mathrm{t}, J=8.0 \mathrm{~Hz}, \mathrm{Ar}), 7.42(1 \mathrm{H}, \mathrm{td}$, $J=8.0,1.7 \mathrm{~Hz}, \mathrm{Ar}), 7.99(1 \mathrm{H}, \mathrm{dd}, J=8.0,1.7 \mathrm{~Hz}, \mathrm{Ar}) ;{ }^{13} \mathrm{C}-\mathrm{NMR}(125 \mathrm{MHz}$, $\left.\mathrm{CDCl}_{3}\right) \delta: 20.6,43.4,44.1,115.2,116.2,123.9,125.5,132.3,142.5,150.4,150.7$; Anal. calcd for $\mathrm{C}_{11} \mathrm{H}_{11} \mathrm{~N}_{3} \mathrm{O}$ : C, 65.66; H, 5.51; N, 20.88. Found: C, 65.55; H, 5.40; $\mathrm{N}, 20.70$.

\subsubsection{Synthesis of 3,4-Dihydro-2H,6H-pyrimido [1,2-c]quinazolin-6(7H)-thione $(8)$}

Xylene (4.0 mL) was added to a flask containing 3,4-dihydro- $2 \mathrm{H}, 6 \mathrm{H}$-pyrimido[1,2-c]quinazolin-6(7H)-one $7(50.3 \mathrm{mg}, 0.25 \mathrm{mmol})$ and Lawesson's reagent (202.2 $\mathrm{mg}, 0.50 \mathrm{mmol})$. After being stirred under reflux for $24 \mathrm{~h}$, xylene $(2 \mathrm{~mL})$ and additional amount of Lawesson's reagent $(101.1 \mathrm{mg}, 0.25 \mathrm{mmol})$ were added. After being stirred under reflux for additional $12 \mathrm{~h}$, the mixture was cooled to $\mathrm{rt}$. The residue was dissolved in $\mathrm{CHCl}_{3}$ and washed with sat. $\mathrm{NaHCO}_{3}$ and brine and dried over $\mathrm{MgSO}_{4}$. After concentration, the residue was purified by flash chromatography over silica gel with $n$-hexane-EtOAc (2:1) to give the title compound 8 as colorless solid (10.4 mg, $19 \%$ ): $\mathrm{mp} 258-259{ }^{\circ} \mathrm{C}$ (from $\mathrm{CHCl}_{3}-n$-hexane); IR (neat) $\mathrm{cm}^{-1}: 1618(\mathrm{C}=\mathrm{N}) ;{ }^{1} \mathrm{H}-\mathrm{NMR}\left(500 \mathrm{MHz}, \mathrm{DMSO}-d_{6}\right) \delta: 1.85-1.90(2 \mathrm{H}, \mathrm{m}$, $\left.\mathrm{CH}_{2}\right), 3.52\left(2 \mathrm{H}, \mathrm{t}, J=5.4 \mathrm{~Hz}, \mathrm{CH}_{2}\right), 4.19\left(2 \mathrm{H}, \mathrm{t}, J=6.0 \mathrm{~Hz}, \mathrm{CH}_{2}\right), 7.12-7.19$ (2H, m, Ar), $7.46(1 \mathrm{H}, \mathrm{t}, J=7.7 \mathrm{~Hz}, \mathrm{Ar}), 7.92(1 \mathrm{H}, \mathrm{d}, J=6.9 \mathrm{~Hz}, \mathrm{Ar}), 12.00(1 \mathrm{H}$, 
s, NH); ${ }^{13} \mathrm{C}-\mathrm{NMR}\left(100 \mathrm{MHz}, \mathrm{DMSO}-d_{6}\right) \delta: 20.6,44.0,46.7,114.8,117.6,123.8$, 125.1, 132.1, 135.9, 142.1, 174.0; HRMS (FAB): $m / z$ calcd for $\mathrm{C}_{11} \mathrm{H}_{12} \mathrm{~N}_{3} \mathrm{~S}$ $[\mathrm{M}+\mathrm{H}]^{+}$218.0752; found: 218.0757 .

\subsubsection{Synthesis of 3,4-Dihydro-2H,6H-pyrimido [1,2-c]quinazolin-6-amine (9)}

2-[N-(p-Toluenesulfonyl)amino]benzaldehyde (13). To a solution of 2-aminobenzylalcohol $12(2.0 \mathrm{~g}, 16.2 \mathrm{mmol})$ and pyridine $(1.6 \mathrm{~mL}, 19.4 \mathrm{mmol})$ in $\mathrm{CHCl}_{3}$ $(60 \mathrm{~mL})$ was added a solution of $p$-TsCl $(3.4 \mathrm{~g}, 18.0 \mathrm{mmol})$ in $\mathrm{CHCl}_{3}(17 \mathrm{~mL})$, and the mixture was stirred at $\mathrm{rt}$ for $3 \mathrm{~h}$. After concentration, EtOAc and sat. $\mathrm{NH}_{4} \mathrm{Cl}$ were added to the residue. The organic phase was separated and dried over $\mathrm{MgSO}_{4}$. After concentration, the resulting solid was added to a suspension of PCC (5.2 g, $24.3 \mathrm{mmol})$ and silica gel $(10.6 \mathrm{~g})$ in $\mathrm{CHCl}_{3}(70 \mathrm{~mL})$. After being stirred at rt for $2 \mathrm{~h}$, the mixture was filtered and concentrated. The residue was purified by flash chromatography over silica gel with $n$-hexane-EtOAc $(9: 1)$ to give the title compound $\mathbf{1 3}$ as colorless solid $\left(3.6 \mathrm{~g}, 80 \%\right.$ ): $\mathrm{mp} 134-136{ }^{\circ} \mathrm{C}$ (from $\mathrm{CHCl}_{3}-n$ hexane); IR (neat) $\mathrm{cm}^{-1}: 1672(\mathrm{C}=\mathrm{O}), 1492\left(\mathrm{NSO}_{2}\right), 1157\left(\mathrm{NSO}_{2}\right) ;{ }^{1} \mathrm{H}-\mathrm{NMR}$ $\left(400 \mathrm{MHz}, \mathrm{CDCl}_{3}\right) \delta: 2.36\left(3 \mathrm{H}, \mathrm{s}, \mathrm{CH}_{3}\right), 7.16(1 \mathrm{H}, \mathrm{t}, J=7.6 \mathrm{~Hz}, \mathrm{Ar}), 7.24(2 \mathrm{H}, \mathrm{d}$, $J=8.5 \mathrm{~Hz}, \mathrm{Ar}), 7.49-7.53(1 \mathrm{H}, \mathrm{m}, \mathrm{Ar}), 7.59(1 \mathrm{H}, \mathrm{dd}, J=7.6,1.5 \mathrm{~Hz}, \mathrm{Ar}), 7.69$ $(1 \mathrm{H}, \mathrm{d}, J=8.3 \mathrm{~Hz}, \mathrm{Ar}), 7.77(2 \mathrm{H}, \mathrm{d}, J=8.5 \mathrm{~Hz}, \mathrm{Ar}), 9.83(1 \mathrm{H}, \mathrm{s}, \mathrm{CHO}), 10.78$ $(1 \mathrm{H}$, br s, $\mathrm{NH}) ;{ }^{13} \mathrm{C}-\mathrm{NMR}\left(100 \mathrm{MHz}, \mathrm{CDCl}_{3}\right) \delta: 21.5,117.8,121.9,122.9,127.3$ (2C), 129.7 (2C), 135.8, 136.1, 136.5, 140.0, 144.1, 194.9; Anal. calcd for $\mathrm{C}_{14} \mathrm{H}_{13} \mathrm{NO}_{3} \mathrm{~S}$ : C, 61.07; H, 4.76; N, 5.09. Found: C, 60.97; H, 4.46; N, 5.05.

2-[2- $N$-(p-Toluenesulfonylamino)phenyl]-1,4,5,6-tetrahydropyrimidine (14).

To a solution of $13(2.75 \mathrm{~g}, 10 \mathrm{mmol})$ in $t$-BuOH $(94 \mathrm{~mL})$ was added propylenediamine (969 mg, $11 \mathrm{mmol}$ ). The mixture was stirred at $70{ }^{\circ} \mathrm{C}$ for $30 \mathrm{~min}$, and then $\mathrm{K}_{2} \mathrm{CO}_{3}(4.15 \mathrm{~g}, 30 \mathrm{mmol})$ and $\mathrm{I}_{2}(3.17 \mathrm{~g}, 12.5 \mathrm{mmol})$ were added. After being stirred at same temperature for $3 \mathrm{~h}$, the mixture was quenched with sat. $\mathrm{Na}_{2} \mathrm{SO}_{3}$ until the iodine color disappeared. The organic layer was separated and concentrated. The resulting solid was dissolved in $\mathrm{H}_{2} \mathrm{O}$. The whole was extracted with $\mathrm{CHCl}_{3}$, and dried over $\mathrm{MgSO}_{4}$. After concentration, the resulting solid was recrystallized from $\mathrm{CHCl}_{3}-$ $\mathrm{Et}_{2} \mathrm{O}-n$-hexane to give the title compound $\mathbf{1 4}$ as pale yellow crystals $(3.23 \mathrm{~g}, 98 \%)$ : mp 211-213 ${ }^{\circ} \mathrm{C}$; IR (neat) $\mathrm{cm}^{-1}: 1630(\mathrm{C}=\mathrm{N}) ; 1478\left(\mathrm{NSO}_{2}\right), 1124\left(\mathrm{NSO}_{2}\right) ;{ }^{1} \mathrm{H}-\mathrm{NMR}$ $\left(400 \mathrm{MHz}, \mathrm{CDCl}_{3}\right) \delta: 1.77-1.82\left(2 \mathrm{H}, \mathrm{m}, \mathrm{CH}_{2}\right), 2.34\left(3 \mathrm{H}, \mathrm{s}, \mathrm{CH}_{3}\right), 3.36(4 \mathrm{H}, \mathrm{t}$, $\left.J=5.7 \mathrm{~Hz}, 2 \times \mathrm{CH}_{2}\right), 6.53-6.57(1 \mathrm{H}, \mathrm{m}, \mathrm{Ar}), 7.04-7.08(1 \mathrm{H}, \mathrm{m}, \mathrm{Ar}), 7.16-7.22$ (3H, m, Ar), $7.58(1 \mathrm{H}, \mathrm{dd}, J=8.2,1.3 \mathrm{~Hz}, \mathrm{Ar}), 7.76(2 \mathrm{H}, \mathrm{d}, J=8.3 \mathrm{~Hz}, \mathrm{Ar}), 10.75$ $\left(1 \mathrm{H}\right.$, br s, NH); ${ }^{13} \mathrm{C}-\mathrm{NMR}\left(100 \mathrm{MHz}, \mathrm{CDCl}_{3}\right) \delta: 18.4,21.3,38.8$ (2C), 112.4, 117.7, 121.2, 126.3 (2C), 126.5, 129.2 (2C), 133.0, 140.9, 142.0, 150.3, 158.9; HRMS (FAB): $m / z$ calcd for $\mathrm{C}_{17} \mathrm{H}_{20} \mathrm{~N}_{3} \mathrm{O}_{2} \mathrm{~S}[\mathrm{M}+\mathrm{H}]^{+} 330.1276$; found: 330.1273 . 
Compound 9. To a flask containing $14(164.7 \mathrm{mg}, 0.5 \mathrm{mmol})$ was added conc. $\mathrm{H}_{2} \mathrm{SO}_{4}(5.0 \mathrm{~mL})$. After being stirred at $100{ }^{\circ} \mathrm{C}$ for $30 \mathrm{~min}$, the mixture was cooled to $0{ }^{\circ} \mathrm{C}$, and then $\mathrm{pH}$ was adjusted to $12-14$ with $2 \mathrm{~N} \mathrm{NaOH}$. The whole was extracted with $\mathrm{CHCl}_{3}$, and dried over $\mathrm{MgSO}_{4}$. After concentration, the residue was dissolved in anhydrous EtOH (2 mL). Then, BrCN (105.9 mg, $1.0 \mathrm{mmol})$ was added to the mixture under an Ar atmosphere. After being stirred under reflux for $2 \mathrm{~h}$, the reaction was quenched with $2 \mathrm{~N} \mathrm{NaOH}$. The whole was extracted with $\mathrm{CHCl}_{3}$, and dried over $\mathrm{MgSO}_{4}$. After concentration, the residue was purified by flash chromatography over aluminum oxide with EtOAc-MeOH (95:5) to give the title compound 9 as colorless solid $(66.0 \mathrm{mg}, 66 \%)$ : $\mathrm{mp} 259-260{ }^{\circ} \mathrm{C}$ (from $\mathrm{CHCl}_{3}-n$-hexane); IR (neat) $\mathrm{cm}^{-1}: 1620 \quad(\mathrm{C}=\mathrm{N}), 1603 \quad(\mathrm{C}=\mathrm{N}) ;{ }^{1} \mathrm{H}-\mathrm{NMR}$ $\left(400 \mathrm{MHz}, \mathrm{DMSO}-d_{6}\right) \delta: 1.81-1.87\left(2 \mathrm{H}, \mathrm{m}, \mathrm{CH}_{2}\right), 3.44\left(2 \mathrm{H}, \mathrm{t}, J=5.4 \mathrm{~Hz}, \mathrm{CH}_{2}\right)$, $3.70\left(2 \mathrm{H}, \mathrm{t}, J=6.1 \mathrm{~Hz}, \mathrm{CH}_{2}\right), 6.49\left(2 \mathrm{H}\right.$, br s, $\left.\mathrm{NH}_{2}\right), 6.87-6.95(2 \mathrm{H}, \mathrm{m}, \mathrm{Ar})$, 7.27-7.31 (1H, m, Ar), $7.87(1 \mathrm{H}, \mathrm{dd}, J=7.9,1.1 \mathrm{~Hz}, \mathrm{Ar}) ;{ }^{13} \mathrm{C}-\mathrm{NMR}(100 \mathrm{MHz}$, DMSO- $\left.d_{6}\right) \delta: 20.0,42.8,42.9,118.9,120.7,122.7,124.3,131.1,145.6,146.6$, 151.6; HRMS (FAB): $\mathrm{m} / z$ calcd for $\mathrm{C}_{11} \mathrm{H}_{13} \mathrm{~N}_{4}[\mathrm{M}+\mathrm{H}]^{+}$201.1140; found: 201.1138 .

\subsubsection{Synthesis of 3,4-Dihydro-2H,6H-pyrimido [1,2-c][1,3]benzothiazin-6-one (11)}

3,4-Dihydro-2H,6H-pyrimido[1,2-c][1,3]benzothiazine-6-thione $\mathbf{1 0} \quad$ (58.6 $\mathrm{mg}$, $0.25 \mathrm{mmol}$ ) was suspended into a $0.1 \mathrm{M} \mathrm{NaOH}$ in $\mathrm{MeOH}-\mathrm{H}_{2} \mathrm{O}(9: 1,5 \mathrm{~mL})$. After being stirred under reflux for $12 \mathrm{~h}$, the mixture was concentrated. To a stirring solution of the residue and $\mathrm{Et}_{3} \mathrm{~N}(0.029 \mathrm{~mL}, 2.0 \mathrm{mmol})$ in $\mathrm{CH}_{2} \mathrm{Cl}_{2}(16.6 \mathrm{~mL})$ was added dropwise a solution of triphosgene $(155.8 \mathrm{mg}, 0.52 \mathrm{mmol})$ in $\mathrm{CH}_{2} \mathrm{Cl}_{2}$ $(1.7 \mathrm{~mL})$ at $0{ }^{\circ} \mathrm{C}$. After being stirred at $\mathrm{rt}$ for $1 \mathrm{~h}$, the mixture was quenched with sat. $\mathrm{NaHCO}_{3}$. The whole was extracted with $\mathrm{CHCl}_{3}$. The extract was washed with sat. $\mathrm{NaHCO}_{3}$, brine, and dried over $\mathrm{MgSO}_{4}$. After concentration, the residue was purified by flash chromatography over silica gel with $n$-hexane-EtOAc (9:1) to give the title compound $\mathbf{1 1}$ as colorless solid (35.3 mg, $65 \%)$ : $\mathrm{mp} 102-103{ }^{\circ} \mathrm{C}$ (from $\mathrm{CHCl}_{3}-n$-hexane); IR (neat) $\mathrm{cm}^{-1}$ : $1639(\mathrm{C}=\mathrm{O}) 1612(\mathrm{C}=\mathrm{N}) ;{ }^{1} \mathrm{H}-\mathrm{NMR}$ $\left(500 \mathrm{MHz} \mathrm{CDCl}_{3}\right) \delta: 1.95-1.99\left(2 \mathrm{H}, \mathrm{m}, \mathrm{CH}_{2}\right), 3.73\left(2 \mathrm{H}, \mathrm{t}, J=5.7 \mathrm{~Hz}, \mathrm{CH}_{2}\right), 4.00$ $\left(2 \mathrm{H}, \mathrm{t}, J=6.0 \mathrm{~Hz}, \mathrm{CH}_{2}\right), 7.13(1 \mathrm{H}, \mathrm{dd}, J=8.0,1.3 \mathrm{~Hz}, \mathrm{Ar}), 7.27-7.30(1 \mathrm{H}, \mathrm{m}$, Ar), $7.40(1 \mathrm{H}, \mathrm{td}, J=8.0,1.1 \mathrm{~Hz}, \mathrm{Ar}), 8.28(1 \mathrm{H}, \mathrm{dd}, J=8.0,1.1 \mathrm{~Hz}, \mathrm{Ar}) ;{ }^{13} \mathrm{C}-$ NMR $\left(125 \mathrm{MHz}, \mathrm{CDCl}_{3}\right) \delta: 20.8,42.4,45.2,124.4,125.8,126.8,128.9,129.2$, $130.9,146.1,162.8$; HRMS (FAB): $m / z$ calcd for $\mathrm{C}_{11} \mathrm{H}_{11} \mathrm{~N}_{2} \mathrm{OS}[\mathrm{M}+\mathrm{H}]^{+}$ 219.0592; found: 219.0592 . 


\subsubsection{Synthesis of 9-(N,N-Dimethylamino)-3,4-dihydro- 2H,6H-pyrimido[1,2-c][1,3]benzothiazin-6-imine (25a)}

$N$-(tert-Butyl)-9-( $N^{\prime}, N^{\prime} H, 6 H$-pyrimido[1,2-c][1,3]benzothiazin-6-imine (22a). To a mixture of $N$-(tert-butyl)-9-bromo-3,4-dihydro- $2 H, 6 H$-pyrimido[1,2c][1,3] benzothiazin-6-imine $22 \mathrm{k}(88.1 \mathrm{mg}, 0.25 \mathrm{mmol})$ and $\mathrm{Pd}(\mathrm{OAc})_{2}(5.6 \mathrm{mg}$, $0.025 \mathrm{mmol})$ and $\mathrm{KO} t$-Bu $(84.2 \mathrm{mg}, 0.75 \mathrm{mmol})$ in toluene $(2.0 \mathrm{~mL})$ were added $\mathrm{P}(\text { tert }-\mathrm{Bu})_{3} \quad(0.009 \mathrm{~mL}, 0.038 \mathrm{mmol})$ and $2 \mathrm{~N} \mathrm{Me}_{2} \mathrm{NH}$ in THF $(0.38 \mathrm{~mL}$, $0.75 \mathrm{mmol}$ ). After being stirred at reflux for $1 \mathrm{~h}$, the mixture was filtered through a Celite pad and concentrated. The residue was purified by flash chromatography over aluminum oxide with $n$-hexane-EtOAc (7:3) to give the title compound 22a as colorless solid ( $80.9 \mathrm{mg},>99 \%$ ): $\mathrm{mp} 161-162{ }^{\circ} \mathrm{C}$ (from $\mathrm{CHCl}_{3}-n$-hexane); IR (neat) $\mathrm{cm}^{-1}: 1587(\mathrm{C}=\mathrm{N}) ;{ }^{1} \mathrm{H}-\mathrm{NMR}\left(400 \mathrm{MHz} \mathrm{CDCl}_{3}\right) \delta: 1.38\left(9 \mathrm{H}, \mathrm{s}, 3 \times \mathrm{CH}_{3}\right)$, 1.86-1.92 (2H, m, $\left.\mathrm{CH}_{2}\right), 2.97\left(6 \mathrm{H}, \mathrm{s}, 2 \times \mathrm{CH}_{3}\right), 3.58\left(2 \mathrm{H}, \mathrm{t}, J=5.5 \mathrm{~Hz}, \mathrm{CH}_{2}\right)$, $3.85\left(2 \mathrm{H}, \mathrm{t}, J=6.1 \mathrm{~Hz}, \mathrm{CH}_{2}\right), 6.28(1 \mathrm{H}, \mathrm{d}, J=2.7 \mathrm{~Hz}, \mathrm{Ar}), 6.55(1 \mathrm{H}, \mathrm{dd}$, $J=9.0,2.7 \mathrm{~Hz}, \mathrm{Ar}), 8.04(1 \mathrm{H}, \mathrm{d}, J=9.0 \mathrm{~Hz}, \mathrm{Ar}) ;{ }^{13} \mathrm{C}-\mathrm{NMR}\left(100 \mathrm{MHz}, \mathrm{CDCl}_{3}\right)$ $\delta: 22.0,30.0$ (3C), 40.0 (2C), 44.9, 45.5, 54.0, 105.5, 110.6, 115.7, 129.7, 130.0, 139.2, 148.0, 151.2; HRMS (FAB): $m / z$ calcd for $\mathrm{C}_{17} \mathrm{H}_{25} \mathrm{~N}_{4} \mathrm{~S}[\mathrm{M}+\mathrm{H}]^{+} 317.1800$; found: 317.1803 .

Compound 25a. TFA $(2.0 \mathrm{~mL})$ was added to a mixture of 22a $(63.3 \mathrm{mg}$, $0.2 \mathrm{mmol}$ ) in small amount of $\mathrm{CHCl}_{3}$ and MS4 $\AA$ (300 mg, powder, activated by heating with Bunsen burner). After being stirred under reflux for $1 \mathrm{~h}$, the mixture was concentrated. To a stirring mixture of the residue in $\mathrm{CHCl}_{3}$ was added dropwise $\mathrm{Et}_{3} \mathrm{~N}$ at $0{ }^{\circ} \mathrm{C}$ to adjust $\mathrm{pH}$ to $8-9$. The whole was extracted with EtOAc. The extract was washed with sat. $\mathrm{NaHCO}_{3}$, brine, and dried over $\mathrm{MgSO}_{4}$. After concentration, the residue was purified by flash chromatography over aluminum oxide with $n$-hexane-EtOAc (7:3) to give the title compound 25a as colorless solid (38.2 mg, $73 \%$ ): $\mathrm{mp} 150-151^{\circ} \mathrm{C}$ (from $\mathrm{CHCl}_{3}-n$-hexane); IR (neat) $\mathrm{cm}^{-1}: 1600$ $(\mathrm{C}=\mathrm{N}), 1562(\mathrm{C}=\mathrm{N}) ;{ }^{1} \mathrm{H}-\mathrm{NMR}\left(500 \mathrm{MHz}, \mathrm{CDCl}_{3}\right) \delta: 1.93-1.98\left(2 \mathrm{H}, \mathrm{m}, \mathrm{CH}_{2}\right)$, $2.98\left(6 \mathrm{H}, \mathrm{s}, 2 \times \mathrm{CH}_{3}\right), 3.64\left(2 \mathrm{H}, \mathrm{t}, J=5.7 \mathrm{~Hz}, \mathrm{CH}_{2}\right), 4.00(2 \mathrm{H}, \mathrm{t}, J=6.3 \mathrm{~Hz}$, $\left.\mathrm{CH}_{2}\right), 6.17(1 \mathrm{H}, \mathrm{d}, J=2.3 \mathrm{~Hz}, \mathrm{Ar}), 6.55(1 \mathrm{H}, \mathrm{dd}, J=9.2,2.3 \mathrm{~Hz}, \mathrm{Ar}), 7.01(1 \mathrm{H}$, br s, NH), $8.05(1 \mathrm{H}, \mathrm{d}, J=9.2 \mathrm{~Hz}, \mathrm{Ar}) ;{ }^{13} \mathrm{C}-\mathrm{NMR}\left(125 \mathrm{MHz}, \mathrm{CDCl}_{3}\right) \delta: 21.1$, 40.0 (2C), 43.8, 44.7, 104.4, 110.7, 114.4, 129.8, 129.9, 146.7, 151.3, 154.2; HRMS (FAB): $m / z$ calcd for $\mathrm{C}_{13} \mathrm{H}_{17} \mathrm{~N}_{4} \mathrm{~S}[\mathrm{M}+\mathrm{H}]^{+}$261.1174; found: 261.1173.

\subsubsection{Synthesis of 3,4-Dihydro-9-(N-methylamino)-2H, 6H-pyrimido[1,2-c][1,3]benzothiazin-6-imine (25b)}

9-Amino- $\mathrm{N}$-(tert-butyl)-3,4-dihydro-2H,6H-pyrimido[1,2-c][1,3]benzothiazin-6imine (28). To a suspension of $\mathrm{N}$-(tert-Butyl)-3,4-dihydro-9-nitro-2 $\mathrm{H}, 6 \mathrm{H}$-pyrimido[1,2-c][1,3]benzothiazin-6-imine 22e (477.0 $\mathrm{mg}, 1.5 \mathrm{mmol})$ in $\mathrm{EtOH}(10 \mathrm{~mL})$ 
was added $10 \% \mathrm{Pd} / \mathrm{C}$ (ca. $55 \%$ in water, $400 \mathrm{mg}$ ) under a $\mathrm{H}_{2}$ atmosphere. After being stirred at $\mathrm{rt}$ overnight, the mixture was filtered through a Celite pad. After concentration, the resulting solid was recrystallized from $\mathrm{CHCl}_{3}-n$-hexane to give the title compound $\mathbf{2 8}$ as colorless crystals (381.1 mg, $88 \%$ ): $\mathrm{mp}$ 152-155 ${ }^{\circ} \mathrm{C}$; IR (neat) $\mathrm{cm}^{-1}: 1589(\mathrm{C}=\mathrm{N}) ;{ }^{1} \mathrm{H}-\mathrm{NMR}\left(400 \mathrm{MHz}, \mathrm{CDCl}_{3}\right) \delta: 1.37$ $\left(9 \mathrm{H}, \mathrm{s}, 3 \times \mathrm{CH}_{3}\right), 1.86-1.92\left(2 \mathrm{H}, \mathrm{m}, \mathrm{CH}_{2}\right), 3.57\left(2 \mathrm{H}, \mathrm{t}, J=5.4 \mathrm{~Hz}, \mathrm{CH}_{2}\right), 3.84$ $\left(2 \mathrm{H}, \mathrm{t}, J=6.0 \mathrm{~Hz}, \mathrm{CH}_{2}\right), 3.88\left(2 \mathrm{H}\right.$, br s, $\left.\mathrm{NH}_{2}\right), 6.33(1 \mathrm{H}, \mathrm{d}, J=2.2 \mathrm{~Hz}, \mathrm{Ar}), 6.49$ $(1 \mathrm{H}, \mathrm{dd}, J=8.5,2.2 \mathrm{~Hz}, \mathrm{Ar}), 7.99(1 \mathrm{H}, \mathrm{d}, J=8.5 \mathrm{~Hz}, \mathrm{Ar}) ;{ }^{13} \mathrm{C}-\mathrm{NMR}(100 \mathrm{MHz}$, $\left.\mathrm{CD}_{3} \mathrm{OD}\right) \delta$ : 22.7, 30.2 (3C), 45.2, 46.8, 55.2, 109.1, 114.4, 116.4, 130.7, 131.4, 140.5, 151.8, 152.4; HRMS (FAB): $m / z$ calcd for $\mathrm{C}_{15} \mathrm{H}_{21} \mathrm{~N}_{4} \mathrm{~S}[\mathrm{M}+\mathrm{H}]^{+} 289.1487$; found: 289.1489 .

$N$-(tert-Butyl)-3,4-dihydro-9-( $N$-methylamino)-2H,6H-pyrimido[1,2-c][1,3] benzothiazin-6-imine (22b). To a flask containing 28 (108.5 mg, $0.38 \mathrm{mmol})$, MeONa (30.6 mg, $0.57 \mathrm{mmol}$ ), and paraformaldehyde (34.2 $\mathrm{mg}, 1.1 \mathrm{mmol}$ ) was added unhydrous $\mathrm{MeOH}(2.5 \mathrm{~mL})$ under an Ar atmosphere, and stirring was continued for $5 \mathrm{~h}$ under reflux. Then, $\mathrm{NaBH}_{4}(28.8 \mathrm{mg}, 0.76 \mathrm{mmol})$ was added to the mixture and stirring was continued for additional 30 min under reflux. After concentration, the residue was dissolved in EtOAc, and washed with sat. $\mathrm{NaHCO}_{3}$, brine, and dried over $\mathrm{MgSO}_{4}$. After concentration, the residue was purified by flash column chromatography over aluminum oxide with $n$-hexane-EtOAc (1:1) to give the title compound $\mathbf{2 2 b}$ as yellow solid (104.9 $\mathrm{mg}, 91 \%$ ): $\mathrm{mp} 156-158{ }^{\circ} \mathrm{C}$ (from $\mathrm{CHCl}_{3}-n$-hexane); IR (neat) $\mathrm{cm}^{-1}: 1590(\mathrm{C}=\mathrm{N}) ;{ }^{1} \mathrm{H}-\mathrm{NMR}\left(400 \mathrm{MHz}, \mathrm{CDCl}_{3}\right) \delta$ : $1.38\left(9 \mathrm{H}, \mathrm{s}, 3 \times \mathrm{CH}_{3}\right), 1.87-1.92\left(2 \mathrm{H}, \mathrm{m}, \mathrm{CH}_{2}\right), 2.83\left(3 \mathrm{H}, \mathrm{s}, \mathrm{CH}_{3}\right), 3.57(2 \mathrm{H}, \mathrm{t}$, $\left.J=5.4 \mathrm{~Hz}, \mathrm{CH}_{2}\right), 3.85\left(2 \mathrm{H}, \mathrm{t}, J=6.0 \mathrm{~Hz}, \mathrm{CH}_{2}\right), 4.04(1 \mathrm{H}, \mathrm{br} \mathrm{s}, \mathrm{NH}), 6.20(1 \mathrm{H}, \mathrm{d}$, $J=2.4 \mathrm{~Hz}, \mathrm{Ar}), 6.43(1 \mathrm{H}, \mathrm{dd}, J=8.8,2.4 \mathrm{~Hz}, \mathrm{Ar}), 8.01(1 \mathrm{H}, \mathrm{d}, J=8.8 \mathrm{~Hz}, \mathrm{Ar})$; ${ }^{13} \mathrm{C}-\mathrm{NMR}\left(100 \mathrm{MHz}, \mathrm{CDCl}_{3}\right.$ ) $\delta: 21.9,30.0$ (3C), 30.2, 44.7, 45.5, 54.0, 104.9, $111.8,116.3,129.7,130.4,139.0,148.3,150.6$; HRMS (FAB): $\mathrm{m} / \mathrm{z}$ calcd for $\mathrm{C}_{16} \mathrm{H}_{23} \mathrm{~N}_{4} \mathrm{~S}[\mathrm{M}+\mathrm{H}]^{+}$303.1643; found: 303.1638 .

Compound 25b. Using the general procedure as described for 25a, compound 22b $(30.7 \mathrm{mg}, 0.1 \mathrm{mmol})$ was allowed to react for $1 \mathrm{~h}$ with TFA $(1.0 \mathrm{~mL})$ and MS4A (200 mg). Purification by flash chromatography over aluminum oxide with $n$-hexane-EtOAc (1:1 to $0: 1)$ gave the title compound $\mathbf{2 5 b}$ as pale yellow solid (9.0 mg, $37 \%$ ): mp $129-131{ }^{\circ} \mathrm{C}$ (from $\mathrm{CHCl}_{3}-n$-hexane); IR (neat) $\mathrm{cm}^{-1}: 1602$ $(\mathrm{C}=\mathrm{N}), 1555(\mathrm{C}=\mathrm{N}) ;{ }^{1} \mathrm{H}-\mathrm{NMR}\left(400 \mathrm{MHz}, \mathrm{CDCl}_{3}\right) \delta: 1.92-1.98\left(2 \mathrm{H}, \mathrm{m}, \mathrm{CH}_{2}\right)$, $2.84\left(3 \mathrm{H}, \mathrm{d}, J=4.1 \mathrm{~Hz}, \mathrm{CH}_{3}\right), 3.64\left(2 \mathrm{H}, \mathrm{t}, J=5.6 \mathrm{~Hz}, \mathrm{CH}_{2}\right), 4.00(2 \mathrm{H}, \mathrm{t}$, $\left.J=6.2 \mathrm{~Hz}, \mathrm{CH}_{2}\right), 4.03(1 \mathrm{H}, \mathrm{br} \mathrm{s}, \mathrm{NH}), 6.11(1 \mathrm{H}, \mathrm{d}, J=2.4 \mathrm{~Hz}, \mathrm{Ar}), 6.44(1 \mathrm{H}, \mathrm{dd}$, $J=8.8,2.4 \mathrm{~Hz}, \mathrm{Ar}), 8.01(1 \mathrm{H}, \mathrm{d}, J=8.8 \mathrm{~Hz}, \mathrm{Ar}) ;{ }^{13} \mathrm{C}-\mathrm{NMR}\left(100 \mathrm{MHz}, \mathrm{CDCl}_{3}\right)$ $\delta: 21.1,30.2$, 43.9, 44.6, 104.0, 111.9, 129.2, 130.1, 130.3, 146.9, 150.8, 154.1; Anal. calcd for $\mathrm{C}_{12} \mathrm{H}_{14} \mathrm{~N}_{4} \mathrm{~S}$ : C, 58.51; H, 5.73; N, 22.74. Found: C, 58.30; H, 5.62; $\mathrm{N}, 22.45$. 


\subsubsection{Synthesis of 9-(N-Acetylamino)-3,4-dihydro-2H, 6H-pyrimido[1,2-c][1,3]benzothiazin-6-imine (25c)}

9-( $N$-Acetylamino)- $N$-(tert-butyl)-3,4-dihydro-2H,6H-pyrimido[1,2-c][1,3]benzothiazin-6-imine (22c). To a mixture of 9-amino- $\mathrm{N}$-(tert-butyl)-3,4-dihydro$2 \mathrm{H}, 6 \mathrm{H}$-pyrimido[1,2-c][1,3]benzothiazin-6-imine 28 (100.9 mg, $0.35 \mathrm{mmol})$, $\mathrm{Et}_{3} \mathrm{~N}(0.015 \mathrm{~mL}, 1.05 \mathrm{mmol}), \mathrm{DMAP}(4.3 \mathrm{mg}, 0.04 \mathrm{mmol})$ in $\mathrm{CH}_{2} \mathrm{Cl}_{2}(3.5 \mathrm{~mL})$ was added $\mathrm{Ac}_{2} \mathrm{O}(0.066 \mathrm{~mL}, 0.70 \mathrm{mmol})$ under an $\mathrm{Ar}$ atmosphere. After being stirred under reflux for $1 \mathrm{~h}$, sat. $\mathrm{NaHCO}_{3}$ was added to the mixture. The whole was extracted with EtOAc. The extract was washed with brine, and dried over $\mathrm{MgSO}_{4}$. After concentration, the residue was purified by flash chromatography over aluminum oxide with $n$-hexane-EtOAc (1:1 to $0: 1)$ to give the title compound 22c as colorless solid (120.1 mg, > $99 \%$ ): mp 213-214 ${ }^{\circ} \mathrm{C}$ (from $\mathrm{CHCl}_{3}-n$-hexane); IR (neat) $\mathrm{cm}^{-1}: 1680(\mathrm{C}=\mathrm{O}), 1596(\mathrm{C}=\mathrm{N}) ;{ }^{1} \mathrm{H}-\mathrm{NMR}\left(400 \mathrm{MHz}, \mathrm{CDCl}_{3}\right) \delta: 1.37(9 \mathrm{H}$, s, $\left.3 \times \mathrm{CH}_{3}\right), 1.88-1.93\left(2 \mathrm{H}, \mathrm{m}, \mathrm{CH}_{2}\right), 2.15\left(3 \mathrm{H}, \mathrm{s}, \mathrm{CH}_{3}\right), 3.59(2 \mathrm{H}, \mathrm{t}, J=5.4 \mathrm{~Hz}$, $\left.\mathrm{CH}_{2}\right), 3.86\left(2 \mathrm{H}, \mathrm{t}, J=6.1 \mathrm{~Hz}, \mathrm{CH}_{2}\right), 6.99(1 \mathrm{H}, \mathrm{dd}, J=8.7,2.1 \mathrm{~Hz}, \mathrm{Ar}), 7.74(1 \mathrm{H}$, $\mathrm{d}, J=2.1 \mathrm{~Hz}, \mathrm{Ar}), 7.96\left(1 \mathrm{H}\right.$, br s, NH), $8.08(1 \mathrm{H}, \mathrm{d}, J=8.7 \mathrm{~Hz}, \mathrm{Ar}) ;{ }^{13} \mathrm{C}-\mathrm{NMR}$ $\left(100 \mathrm{MHz}, \mathrm{CDCl}_{3}\right) \delta: 21.8,24.6,29.9$ (3C), 44.9, 45.4, 54.2, 114.4, 116.8, 123.1, 129.1, 130.4, 138.2, 139.7, 147.8, 168.6; HRMS (FAB): $m / z$ calcd for $\mathrm{C}_{17} \mathrm{H}_{23} \mathrm{~N}_{4} \mathrm{OS}[\mathrm{M}+\mathrm{H}]^{+}$331.1593; found: 331.1590 .

Compound 25c. Using the general procedure as described for 28a, compound 25c $(120.1 \mathrm{mg}, 0.36 \mathrm{mmol}$ ) was allowed to react for $10 \mathrm{~h}$. Purification by recrystallization from $\mathrm{MeOH}-\mathrm{CHCl}_{3}-\mathrm{Et}_{2} \mathrm{O}$ gave the title compound $25 \mathrm{c}$ as pale yellow crystals $(64.9 \mathrm{mg}, 65 \%)$ : $\mathrm{mp} 214{ }^{\circ} \mathrm{C}$ (decomp.); IR (neat) $\mathrm{cm}^{-1}$ : 1681 $(\mathrm{C}=\mathrm{O}), 1619(\mathrm{C}=\mathrm{N}), 1550(\mathrm{C}=\mathrm{N}) ;{ }^{1} \mathrm{H}-\mathrm{NMR}\left(400 \mathrm{MHz}, \mathrm{DMSO}-d_{6}\right) \delta: 1.85-1.91$ $\left(2 \mathrm{H}, \mathrm{m}, \mathrm{CH}_{2}\right), 2.07\left(3 \mathrm{H}, \mathrm{s}, \mathrm{CH}_{3}\right), 3.55\left(2 \mathrm{H}, \mathrm{t}, J=5.5 \mathrm{~Hz}, \mathrm{CH}_{2}\right), 3.92(2 \mathrm{H}, \mathrm{t}$, $\left.J=6.0 \mathrm{~Hz}, \mathrm{CH}_{2}\right), 7.32(1 \mathrm{H}, \mathrm{dd}, J=8.9,1.8 \mathrm{~Hz}, \mathrm{Ar}), 7.61(1 \mathrm{H}, \mathrm{d}, J=1.8 \mathrm{~Hz}$, Ar), $8.10(1 \mathrm{H}, \mathrm{d}, J=8.9 \mathrm{~Hz}, \mathrm{Ar}), 9.14\left(1 \mathrm{H}\right.$, br s, NH), $10.27(1 \mathrm{H}, \mathrm{s}, \mathrm{NH}) ;{ }^{13} \mathrm{C}-$ NMR (100 MHz, DMSO- $\left.d_{6}\right) \delta: 20.3,24.1,43.3,43.6,112.2,116.7,119.3,129.2$, 129.9, 141.7, 146.3, 149.5, 169.0; HRMS (FAB): $\mathrm{m} / \mathrm{z}$ calcd for $\mathrm{C}_{13} \mathrm{H}_{15} \mathrm{~N}_{4} \mathrm{OS}$ $[\mathrm{M}+\mathrm{H}]^{+}$275.0967; found: 275.0967 .

\subsubsection{Synthesis of 9-Acetyl-3,4-dihydro-2H, 6H-pyrimido[1,2-c][1,3]benzothiazin-6-imine (25d)}

To a mixture of $N$-(tert-butyl)-9-bromo-3,4-dihydro-2 $H, 6 H$-pyrimido[1,2-c] [1,3]benzothiazin-6-imine $\mathbf{2 2 k} \quad(100 \mathrm{mg}, \quad 0.284 \mathrm{mmol}), \quad \mathrm{Pd}(\mathrm{OAc})_{2} \quad(6.4 \mathrm{mg}$, $0.0284 \mathrm{mmol}), \mathrm{K}_{2} \mathrm{CO}_{3}(120 \mathrm{mg}, 0.852 \mathrm{mmol})$ and dppp (23.7 $\left.\mathrm{mg}, 0.0568 \mathrm{mmol}\right)$ in $\mathrm{H}_{2} \mathrm{O}(0.57 \mathrm{~mL})$ was added ethylene glycol monovinyl ether $(0.13 \mathrm{~mL}$, $1.42 \mathrm{mmol}$ ). After being stirred at reflux for $12 \mathrm{~h}$, the whole was extracted with $\mathrm{CHCl}_{3}$. The extract was washed with brine, and dried over $\mathrm{Na}_{2} \mathrm{SO}_{4}$. After concentration, TFA $(2.84 \mathrm{~mL})$ was added to resulting residue. After being stirred under reflux for $1.5 \mathrm{~h}$, the mixture was added dropwise to $\mathrm{Et}_{3} \mathrm{~N}$ at $0{ }^{\circ} \mathrm{C}$ to adjust 
$\mathrm{pH}$ to 8-9. The whole was extracted with EtOAc. The extract was washed with brine, and dried over $\mathrm{Na}_{2} \mathrm{SO}_{4}$. After concentration, the residue was purified by preparative TLC over aluminum oxide with $n$-hexane-EtOAc $(7: 3)$ to give the title compound 25d as pale yellow solid $(9.7 \mathrm{mg}, 13 \%)$ : $\mathrm{mp} 148.4{ }^{\circ} \mathrm{C}$; IR (neat) $\mathrm{cm}^{-1}$ : $1678(\mathrm{C}=\mathrm{O}), 1616(\mathrm{C}=\mathrm{N}), 1567(\mathrm{C}=\mathrm{N}) ;{ }^{1} \mathrm{H}-\mathrm{NMR}\left(300 \mathrm{MHz}, \mathrm{CDCl}_{3}\right) \delta: 1.95-2.03$ $\left(2 \mathrm{H}, \mathrm{m}, \mathrm{CH}_{2}\right), 2.60\left(3 \mathrm{H}, \mathrm{s}, \mathrm{CH}_{3}\right), 3.72\left(2 \mathrm{H}, \mathrm{t}, J=5.7 \mathrm{~Hz}, \mathrm{CH}_{2}\right), 4.03(2 \mathrm{H}, \mathrm{t}$, $\left.J=6.3 \mathrm{~Hz}, \mathrm{CH}_{2}\right), 7.63(1 \mathrm{H}, \mathrm{d}, J=1.8 \mathrm{~Hz}, \mathrm{Ar}), 7.74(1 \mathrm{H}, \mathrm{dd}, J=8.3,1.7 \mathrm{~Hz}$, Ar), $8.32(1 \mathrm{H}, \mathrm{d}, J=7.8 \mathrm{~Hz}, \mathrm{Ar}) ;{ }^{13} \mathrm{C}-\mathrm{NMR}\left(75 \mathrm{MHz}, \mathrm{CDCl}_{3}\right) \delta: 20.9,26.7,43.8$, 45.1, 123.6, 125.7, 129.3, 129.6, 130.4, 138.3, 146.0, 152.6, 196.7; Anal. calcd for $\mathrm{C}_{13} \mathrm{H}_{13} \mathrm{~N}_{3} \mathrm{OS}$ : C, 60.21; H, 5.05; N, 16.20. Found: C, 60.16; H, 5.02; N, 15.94.

\subsubsection{Synthesis of 3,4-Dihydro-9-nitro-2H, 6H-pyrimido[1,2-c][1,3]benzothiazin-6-imine (25e)}

\section{$N$-(tert-Butyl)-3,4-dihydro-9-nitro-2H,6H-pyrimido[1,2-c][1,3]benzothiazin-6-}

imine (22e). To a mixture of 2-(2-fluoro-4-nitrophenyl)-1,4,5,6-tetrahydropyrimidine 18e (2.0 g, $9.0 \mathrm{mmol})$ and $\mathrm{NaH}(716.8 \mathrm{mg}, 17.9 \mathrm{mmol} ; 60 \%$ oil suspension) in DMF $(29.8 \mathrm{~mL})$ was added tert-butylisothiocyanate $(2.28 \mathrm{~mL}, 17.9 \mathrm{mmol})$ under an Ar atmosphere, and the mixture was stirred at $-20{ }^{\circ} \mathrm{C}$ to $\mathrm{rt}$ for 2 days. The whole was extracted with EtOAc, and the extract was washed with sat. $\mathrm{NaHCO}_{3}$, brine, and dried over $\mathrm{Na}_{2} \mathrm{SO}_{4}$. After concentration, the residue was purified by flash chromatography over aluminum oxide with $n$-hexane-EtOAc (1:0 to 9:1) to give the title compound $22 \mathrm{e}$ as pale yellow solid (1.77 $\mathrm{g}, 62 \%)$ : $\mathrm{mp}$ $152-153{ }^{\circ} \mathrm{C}$ (from $\mathrm{CHCl}_{3}-n$-hexane); IR (neat) $\mathrm{cm}^{-1}: 1591\left(\mathrm{NO}_{2}\right), 1581(\mathrm{C}=\mathrm{N})$, $1523\left(\mathrm{NO}_{2}\right) ;{ }^{1} \mathrm{H}-\mathrm{NMR}\left(500 \mathrm{MHz}, \mathrm{CDCl}_{3}\right) \delta: 1.39\left(9 \mathrm{H}, \mathrm{s}, 3 \times \mathrm{CH}_{3}\right), 1.91-1.96$ $\left(2 \mathrm{H}, \mathrm{m}, \mathrm{CH}_{2}\right), 3.66\left(2 \mathrm{H}, \mathrm{t}, J=5.2 \mathrm{~Hz}, \mathrm{CH}_{2}\right), 3.88\left(2 \mathrm{H}, \mathrm{t}, J=5.7 \mathrm{~Hz}, \mathrm{CH}_{2}\right), 7.97$ $(1 \mathrm{H}, \mathrm{dd}, J=9.7,2.3 \mathrm{~Hz}, \operatorname{Ar}), 8.01(1 \mathrm{H}, \mathrm{d}, J=2.3 \mathrm{~Hz}, \operatorname{Ar}), 8.39(1 \mathrm{H}, \mathrm{d}$, $J=9.2 \mathrm{~Hz}, \mathrm{Ar}) ;{ }^{13} \mathrm{C}-\mathrm{NMR}\left(125 \mathrm{MHz}, \mathrm{CDCl}_{3}\right) \delta: 21.7,30.0$ (3C), 45.3, 45.5, 54.5, 119.9, 120.3, 130.0, 131.1, 132.8, 136.1, 146.5, 148.5; HRMS (FAB): $m / z$ calcd for $\mathrm{C}_{15} \mathrm{H}_{19} \mathrm{~N}_{4} \mathrm{O}_{2} \mathrm{~S}[\mathrm{M}+\mathrm{H}]^{+}$319.1229; found: 319.1229 .

Compound 25e. Using the general procedure as described for 25a, compound 22e $(47.8 \mathrm{mg}, 0.15 \mathrm{mmol})$ was allowed to react for $1 \mathrm{~h}$ with TFA $(1.5 \mathrm{~mL})$ and MS4A (225 mg). Purification by flash chromatography over aluminum oxide with $n$-hexane-EtOAc (19:1 to 1:1) gave the title compound 25e as pale yellow solid (24.9 mg, $63 \%$ ): $\mathrm{mp} 170-172{ }^{\circ} \mathrm{C}$ (from $\mathrm{CHCl}_{3}-n$-hexane); IR (neat) $\mathrm{cm}^{-1}: 1620$ $(\mathrm{C}=\mathrm{N}), 1587\left(\mathrm{NO}_{2}\right), 1568(\mathrm{C}=\mathrm{N}), 1523\left(\mathrm{NO}_{2}\right) ;{ }^{1} \mathrm{H}-\mathrm{NMR}\left(400 \mathrm{MHz}, \mathrm{CDCl}_{3}\right) \delta$ : 1.97-2.03 (2H, m, $\left.\mathrm{CH}_{2}\right), 3.74\left(2 \mathrm{H}, \mathrm{t}, J=5.6 \mathrm{~Hz}, \mathrm{CH}_{2}\right), 4.04(2 \mathrm{H}, \mathrm{t}, J=6.2 \mathrm{~Hz}$, $\left.\mathrm{CH}_{2}\right), 7.41(1 \mathrm{H}$, br s, NH), $7.93(1 \mathrm{H}, \mathrm{d}, J=2.2 \mathrm{~Hz}, \mathrm{Ar}), 8.00(1 \mathrm{H}, \mathrm{dd}, J=9.0$, $2.2 \mathrm{~Hz}, \mathrm{Ar}), 8.42(1 \mathrm{H}, \mathrm{d}, J=9.0 \mathrm{~Hz}, \mathrm{Ar}) ;{ }^{13} \mathrm{C}-\mathrm{NMR}\left(100 \mathrm{MHz}, \mathrm{CDCl}_{3}\right) \delta: 20.8$, 43.8, 45.2, 118.9, 120.5, 130.4, 130.8, 131.7, 145.1, 148.7, 151.3; Anal. calcd for $\mathrm{C}_{11} \mathrm{H}_{10} \mathrm{~N}_{4} \mathrm{O}_{2} \mathrm{~S}$ : C, 50.37; H, 3.84; N, 21.36. Found: C, 50.29; H, 4.03; N, 21.08. 


\subsubsection{Synthesis of 3,4-Dihydro-9-methoxy-2H,6H- pyrimido[1,2-c][1,3]benzothiazin-6-imine (25f)}

3,4-Dihydro-9-methoxy-2H,6H-pyrimido[1,2-c][1,3]benzothiazine-6-thione $21 f$ (66.1 mg, $0.25 \mathrm{mmol}$ ) was suspended into a $0.1 \mathrm{M} \mathrm{NaOH}$ in $\mathrm{MeOH}-\mathrm{H}_{2} \mathrm{O}$ (9:1) $(5 \mathrm{~mL})$, and the mixture was stirred for $12 \mathrm{~h}$ under reflux. After concentration, the residue was suspended in anhydrous EtOH $(1 \mathrm{~mL})$. BrCN $(53.0 \mathrm{mg}, 0.50 \mathrm{mmol})$ was added under an Ar atmosphere, and the mixture was stirred for $2 \mathrm{~h}$ under reflux. The reaction was quenched with $2 \mathrm{~N} \mathrm{NaOH}$, and the whole was extracted with $\mathrm{CHCl}_{3}$, and dried over $\mathrm{MgSO}_{4}$. After concentration, the residue was purified by flash chromatography over aluminum oxide with $n$-hexane-EtOAc $(9: 1)$ to give the title compound $\mathbf{2 5 f}$ as colorless solid (37.6 mg, 61\%): $\mathrm{mp} 106{ }^{\circ} \mathrm{C}$ (from $\mathrm{CHCl}_{3}-n$-hexane); IR (neat) $\mathrm{cm}^{-1}: 1620(\mathrm{C}=\mathrm{N}), 1572 \quad(\mathrm{C}=\mathrm{N}) ;{ }^{1} \mathrm{H}-\mathrm{NMR}$ $\left(500 \mathrm{MHz} \mathrm{CDCl}_{3}\right) \delta: 1.94-1.98\left(2 \mathrm{H}, \mathrm{m}, \mathrm{CH}_{2}\right), 3.66\left(2 \mathrm{H}, \mathrm{t}, J=5.7 \mathrm{~Hz}, \mathrm{CH}_{2}\right)$, $3.81\left(3 \mathrm{H}, \mathrm{s}, \mathrm{CH}_{3}\right), 4.01\left(2 \mathrm{H}, \mathrm{t}, J=6.0 \mathrm{~Hz}, \mathrm{CH}_{2}\right), 6.50(1 \mathrm{H}, \mathrm{d}, J=2.3 \mathrm{~Hz}, \mathrm{Ar})$, $6.76(1 \mathrm{H}, \mathrm{dd}, J=9.0,2.3 \mathrm{~Hz}, \mathrm{Ar}), 7.15(1 \mathrm{H}, \mathrm{br}, \mathrm{NH}), 8.15(1 \mathrm{H}, \mathrm{d}, J=9.0 \mathrm{~Hz}$, $\mathrm{Ar}) ;{ }^{13} \mathrm{C}-\mathrm{NMR}\left(125 \mathrm{MHz}, \mathrm{CDCl}_{3}\right) \delta: 21.0,43.8,44.8,55.5,107.3,113.3,119.5$, 130.2, 130.6, 146.2, 153.4, 161.2; Anal. calcd for $\mathrm{C}_{12} \mathrm{H}_{13} \mathrm{~N}_{3} \mathrm{OS}$ : C, 58.28; H, 5.30; N, 16.99. Found: C, 58.15; H, 5.23; N, 16.79 .

\subsubsection{Synthesis of 3,4-Dihydro-9-methyl-2H, 6H-pyrimido[1,2-c][1,3]benzothiazin-6-imine (25g)}

3,4-Dihydro-9-methyl-2H,6H-pyrimido[1,2-c][1,3]benzothiazine-6-thione $\quad \mathbf{2 1 g}$ (62.1 $\mathrm{mg}, 0.25 \mathrm{mmol}$ ) was subjected to the general procedure as described for $\mathbf{2 5 f}$ to give the title compound $\mathbf{2 5 g}$ as colorless solid (39.2 $\mathrm{mg}, 68 \%$ ): $\mathrm{mp} 121{ }^{\circ} \mathrm{C}$ (from $\mathrm{CHCl}_{3}-n$-hexane); IR (neat) $\mathrm{cm}^{-1}$ : $1620(\mathrm{C}=\mathrm{N}), 1569(\mathrm{C}=\mathrm{N})$; ${ }^{1} \mathrm{H}-\mathrm{NMR}$ $\left(500 \mathrm{MHz}, \mathrm{CDCl}_{3}\right) \delta:$ 1.94-1.99 $\left(2 \mathrm{H}, \mathrm{m}, \mathrm{CH}_{2}\right), 2.32\left(3 \mathrm{H}, \mathrm{s}, \mathrm{CH}_{3}\right), 3.67(2 \mathrm{H}, \mathrm{t}$, $\left.J=5.7 \mathrm{~Hz}, \mathrm{CH}_{2}\right), 4.01\left(2 \mathrm{H}, \mathrm{t}, J=6.3 \mathrm{~Hz}, \mathrm{CH}_{2}\right), 6.84(1 \mathrm{H}, \mathrm{s}, \mathrm{Ar}), 7.02(1 \mathrm{H}, \mathrm{d}$, $J=8.6 \mathrm{~Hz}, \mathrm{Ar}), 7.16\left(1 \mathrm{H}\right.$, br s, NH), $8.10(1 \mathrm{H}, \mathrm{d}, J=8.6 \mathrm{~Hz}, \mathrm{Ar}) ;{ }^{13} \mathrm{C}-\mathrm{NMR}$ $\left(125 \mathrm{MHz}, \mathrm{CDCl}_{3}\right) \delta: 21.1,21.1,43.8,44.9,123.6,124.1,127.4,128.6,128.8$, 141.1, 146.6, 153.6; HRMS (FAB): $m / z$ calcd for $\mathrm{C}_{12} \mathrm{H}_{14} \mathrm{~N}_{3} \mathrm{~S}[\mathrm{M}+\mathrm{H}]^{+} 232.0908$; found: 232.0912 .

\subsubsection{Synthesis of 9-(n-Butyl)-3,4-dihydro-2H, 6H-pyrimido[1,2-c][1,3]benzothiazin-6-imine (25h)}

9-(n-Butyl)- $N$-(tert-butyl)-3,4-dihydro-2H, $6 H$-pyrimido[1,2-c $][1,3]$ benzothiazin-6-imine (22h). To a mixture of $N$-(tert-butyl)-9-bromo-3,4-dihydro$2 H, 6 H$-pyrimido[1,2-c][1,3]benzothiazin-6-imine 22k $(352.3 \mathrm{mg}, 1.0 \mathrm{mmol})$, 
n-butylboronic acid (152.9 mg, $1.5 \mathrm{mmol}), \mathrm{Pd}_{2}(\mathrm{dba})_{3} \cdot \mathrm{CHCl}_{3} \quad(51.8 \mathrm{mg}$, $0.05 \mathrm{mmol})$ and $\mathrm{Cs}_{2} \mathrm{CO}_{3}(391.0 \mathrm{mg}, 1.2 \mathrm{mmol})$ in 1,4-dioxane $(2.5 \mathrm{~mL})$ was added $\mathrm{P}(\text { tert }-\mathrm{Bu})_{3}(0.024 \mathrm{~mL}, 0.1 \mathrm{mmol})$ under an $\mathrm{Ar}$ atmosphere, the mixture was stirred for $19 \mathrm{~h}$ under reflux. The mixture was filtered through a Celite pad, and concentrated. The residue was purified by flash chromatography over aluminum oxide with $n$-hexane-EtOAc (1:0 to 19:1) to give the title compound 22h as a colorless oil $(21.0 \mathrm{mg}, 6 \%)$ : IR (neat) $\mathrm{cm}^{-1}: 1593(\mathrm{C}=\mathrm{N}) ;{ }^{1} \mathrm{H}$ NMR (400 MHz, $\left.\mathrm{CDCl}_{3}\right) \delta ; 0.91\left(3 \mathrm{H}, \mathrm{t}, J=7.3 \mathrm{~Hz}, \mathrm{CH}_{3}\right), 1.29-1.36(2 \mathrm{H}, \mathrm{m}$, $\left.\mathrm{CH}_{2}\right), 1.38\left(9 \mathrm{H}, \mathrm{s}, 3 \times \mathrm{CH}_{3}\right), 1.54-1.62\left(2 \mathrm{H}, \mathrm{m}, \mathrm{CH}_{2}\right), 1.87-1.93(2 \mathrm{H}, \mathrm{m}$, $\left.\mathrm{CH}_{2}\right), 2.57\left(2 \mathrm{H}, \mathrm{t}, J=7.7 \mathrm{~Hz}, \mathrm{CH}_{2}\right), 3.60\left(2 \mathrm{H}, \mathrm{t}, J=5.4 \mathrm{~Hz}, \mathrm{CH}_{2}\right), 3.86(2 \mathrm{H}$, $\left.\mathrm{t}, J=6.0 \mathrm{~Hz}, \mathrm{CH}_{2}\right), 6.91(1 \mathrm{H}, \mathrm{d}, J=1.2 \mathrm{~Hz}, \mathrm{Ar}), 7.01(1 \mathrm{H}, \mathrm{dd}, J=8.3$, $1.2 \mathrm{~Hz}, \mathrm{Ar}), 8.08(1 \mathrm{H}, \mathrm{d}, J=8.3 \mathrm{~Hz}, \mathrm{Ar}) ;{ }^{13} \mathrm{C}-\mathrm{NMR}\left(100 \mathrm{MHz}, \mathrm{CDCl}_{3}\right) \delta$ : 13.8, 21.9, 22.2, 30.0 (3C), 33.0, 35.2, 45.0, 45.4, 54.1, 123.9, 125.3, 126.5, 128.3, 128.7, 138.6, 145.4, 147.9; HRMS (FAB): $m / z$ calcd for $\mathrm{C}_{19} \mathrm{H}_{28} \mathrm{~N}_{3} \mathrm{~S}$ $[\mathrm{M}+\mathrm{H}]^{+}$330.2004; found: 330.1999 .

Compound 25h. Using the general procedure as described for 25a, compound 22h $(10.3 \mathrm{mg}, 0.03 \mathrm{mmol})$ was allowed to react for $1 \mathrm{~h}$ with TFA $(1.0 \mathrm{~mL})$ and MS4 A (150 mg). Purification by flash chromatography over aluminum oxide with n-hexane-EtOAc (9:1) gave the title compound 25h as colorless solid (5.2 $\mathrm{mg}$, $61 \%): \operatorname{mp~} 52-55{ }^{\circ} \mathrm{C}$ (from $n$-hexane); IR (neat) $\mathrm{cm}^{-1}: 1621(\mathrm{C}=\mathrm{N}), 1571(\mathrm{C}=\mathrm{N})$; ${ }^{1} \mathrm{H}-\mathrm{NMR}\left(400 \mathrm{MHz}, \mathrm{CDCl}_{3}\right) \delta ; 0.91\left(3 \mathrm{H}, \mathrm{t}, J=7.3 \mathrm{~Hz}, \mathrm{CH}_{3}\right), 1.29-1.38(2 \mathrm{H}, \mathrm{m}$, $\left.\mathrm{CH}_{2}\right), 1.54-1.61\left(2 \mathrm{H}, \mathrm{m}, \mathrm{CH}_{2}\right), 1.93-1.99\left(2 \mathrm{H}, \mathrm{m}, \mathrm{CH}_{2}\right), 2.58(2 \mathrm{H}, \mathrm{t}, J=7.7 \mathrm{~Hz}$, $\left.\mathrm{CH}_{2}\right), 3.67\left(2 \mathrm{H}, \mathrm{t}, J=5.6 \mathrm{~Hz}, \mathrm{CH}_{2}\right), 4.01\left(2 \mathrm{H}, \mathrm{t}, J=6.2 \mathrm{~Hz}, \mathrm{CH}_{2}\right), 6.84(1 \mathrm{H}, \mathrm{d}$, $J=1.5 \mathrm{~Hz}, \mathrm{Ar}), 7.03-7.05(1 \mathrm{H}, \mathrm{m}, \mathrm{Ar}), 8.11(1 \mathrm{H}, \mathrm{d}, J=8.3 \mathrm{~Hz}, \mathrm{Ar}) ;{ }^{13} \mathrm{C}-\mathrm{NMR}$ $\left(100 \mathrm{MHz}, \mathrm{CDCl}_{3}\right) \delta: 13.8,21.1,22.2,33.0,35.2,43.8,44.9,123.0,124.4,126.8$, 128.6, 128.8, 146.1, 146.6, 153.7; HRMS (FAB): $m / z$ calcd for $\mathrm{C}_{15} \mathrm{H}_{20} \mathrm{~N}_{3} \mathrm{~S}$ $[\mathrm{M}+\mathrm{H}]^{+}$274.1378; found: 274.1372.

\subsubsection{Synthesis of 9-Fluoro-3,4-dihydro-2H, 6H-pyrimido[1,2-c][1,3]benzothiazin-6-imine (25i)}

9-Fluoro-3,4-dihydro-2H,6H-pyrimido[1,2-c][1,3]benzothiazine-6-thione $\quad \mathbf{2 1 i}$ (63.1 $\mathrm{mg}, 0.25 \mathrm{mmol}$ ) was subjected to the general procedure as described for $\mathbf{2 5 f}$ to give the title compound $\mathbf{2 5 i}$ as colorless solid (30.4 mg, $52 \%): \mathrm{mp} 123-124{ }^{\circ} \mathrm{C}$ (from $\mathrm{CHCl}_{3}-n$-hexane); IR (neat) $\mathrm{cm}^{-1}$ : $1624(\mathrm{C}=\mathrm{N}), 1585(\mathrm{C}=\mathrm{N}) ;{ }^{1} \mathrm{H}-\mathrm{NMR}$ $\left(500 \mathrm{MHz}, \mathrm{CDCl}_{3}\right) \delta: 1.94-2.00\left(2 \mathrm{H}, \mathrm{m}, \mathrm{CH}_{2}\right), 3.67\left(2 \mathrm{H}, \mathrm{t}, J=5.7 \mathrm{~Hz}, \mathrm{CH}_{2}\right)$, $4.01\left(2 \mathrm{H}, \mathrm{t}, J=6.3 \mathrm{~Hz}, \mathrm{CH}_{2}\right), 6.75(1 \mathrm{H}, \mathrm{dd}, J=8.0,2.9 \mathrm{~Hz}, \mathrm{Ar}), 6.91(1 \mathrm{H}, \mathrm{ddd}$, $J=8.6,8.0,2.9 \mathrm{~Hz}, \mathrm{Ar}), 7.22(1 \mathrm{H}$, br s, NH), $8.24(1 \mathrm{H}, \mathrm{dd}, J=8.6,5.7 \mathrm{~Hz}, \mathrm{Ar})$; ${ }^{13} \mathrm{C}-\mathrm{NMR}\left(125 \mathrm{MHz}, \mathrm{CDCl}_{3}\right) \delta: 21.0,43.8,44.8,110.0(\mathrm{~d}, J=25.2 \mathrm{~Hz}), 113.9$ $(\mathrm{d}, J=21.6 \mathrm{~Hz}), 123.1,130.9(\mathrm{~d}, J=8.4 \mathrm{~Hz}), 131.5(\mathrm{~d}, J=8.4 \mathrm{~Hz}), 146.4(\mathrm{~d}$, $J=155.9 \mathrm{~Hz}), 152.6,163.7(\mathrm{~d}, J=254.3 \mathrm{~Hz}) ;{ }^{19} \mathrm{~F}-\mathrm{NMR}\left(500 \mathrm{MHz}, \mathrm{CDCl}_{3}\right) \delta$ : -109.1; Anal. calcd for $\mathrm{C}_{11} \mathrm{H}_{10} \mathrm{FN}_{3} \mathrm{~S}$ : C, 56.15; H, 4.28; N, 17.86. Found: C, 56.13; H, 4.44; N, 17.78 . 


\subsubsection{Synthesis of 3,4-Dihydro-9-trifluoromethyl-2H, 6H-pyrimido[1,2-c][1,3]benzothiazin-6-imine (25j)}

2-(2-Fluoro-4-trifluoromethylphenyl)-1,4,5,6-tetrahydropyrimidine (18j). To a solution of 2-fluoro-4-trifluoromethylbenzaldehyde $\mathbf{1 5} \mathbf{j}(1.00 \mathrm{~g}, 5.21 \mathrm{mmol})$ in $t$ $\mathrm{BuOH}(49 \mathrm{~mL})$ was added propylenediamine $(424.7 \mathrm{mg}, 5.73 \mathrm{mmol})$. The mixture was stirred at $70{ }^{\circ} \mathrm{C}$ for $30 \mathrm{~min}$, and then $\mathrm{K}_{2} \mathrm{CO}_{3}(2.16 \mathrm{~g}, 15.6 \mathrm{mmol})$ and $\mathrm{I}_{2}$ ( $1.65 \mathrm{~g}, 6.51 \mathrm{mmol})$ were added. After being stirred at same temperature for $3 \mathrm{~h}$, the mixture was quenched with sat. $\mathrm{Na}_{2} \mathrm{SO}_{3}$. The organic layer was separated and concentrated. The resulting solid was dissolved with $\mathrm{H}_{2} \mathrm{O}$, and then $\mathrm{pH}$ was adjusted to 12-14 with $2 \mathrm{~N} \mathrm{NaOH}$. The whole was extracted with $\mathrm{CHCl}_{3}$, and the extract was dried over $\mathrm{MgSO}_{4}$. After concentration, the resulting solid was recrystallized from $\mathrm{CHCl}_{3}-n$-hexane to give the title compound $18 \mathrm{j}$ as colorless crystals $(0.84 \mathrm{~g}, 65 \%)$ : mp $108-110{ }^{\circ} \mathrm{C}$; IR (neat) $\mathrm{cm}^{-1}: 1620(\mathrm{C}=\mathrm{N}) ;{ }^{1} \mathrm{H}-\mathrm{NMR}$ $\left(500 \mathrm{MHz}, \mathrm{CDCl}_{3}\right) \delta: 1.86-1.90\left(2 \mathrm{H}, \mathrm{m}, \mathrm{CH}_{2}\right), 3.52(4 \mathrm{H}, \mathrm{t}, J=5.2 \mathrm{~Hz}$, $\left.2 \times \mathrm{CH}_{2}\right), 5.34(1 \mathrm{H}$, br s, NH), $7.33(1 \mathrm{H}, \mathrm{d}, J=11.5 \mathrm{~Hz}, \mathrm{Ar}), 7.42(1 \mathrm{H}, \mathrm{d}$, $J=8.6 \mathrm{~Hz}, \mathrm{Ar}), 7.96(1 \mathrm{H}, \mathrm{dd}, J=8.6,8.0 \mathrm{~Hz}, \mathrm{Ar}) ;{ }^{13} \mathrm{C}-\mathrm{NMR}(100 \mathrm{MHz}$, $\left.\mathrm{CDCl}_{3}\right) \delta: 20.5,42.2(2 \mathrm{C}), 113.4(\mathrm{dq}, J=26.9,3.9 \mathrm{~Hz}), 120.9-121.0(\mathrm{~m}), 123.0$ $(\mathrm{dq}, J=273.0,2.5 \mathrm{~Hz}), 128.0(\mathrm{~d}, J=13.2 \mathrm{~Hz}), 131.5(\mathrm{~d}, J=4.1 \mathrm{~Hz}), 132.8(\mathrm{dq}$, $J=33.7,9.1 \mathrm{~Hz}), 150.4,159.6(\mathrm{~d}, J=249.1 \mathrm{~Hz}) ;{ }^{19} \mathrm{~F}-\mathrm{NMR}\left(500 \mathrm{MHz}, \mathrm{CDCl}_{3}\right)$ $\delta$ : -115.1, -63.4; Anal. calcd for $\mathrm{C}_{11} \mathrm{H}_{10} \mathrm{~F}_{4} \mathrm{~N}_{2}$ : C, 53.66; H, 4.09; N, 11.38. Found: C, 53.82; H, 4.06; N, 11.43.

$N$-(tert-Butyl)-3,4-dihydro-9-trifluoromethyl-2H, $6 H$-pyrimido[1,2-c][1,3] benzothiazin-6-imine $\mathbf{( 2 2} \mathbf{j})$. Using the general procedure as described for $\mathbf{2 2 e}$, compound 18j (246.2 mg, $1.0 \mathrm{mmol}$ ) was allowed to react at $80{ }^{\circ} \mathrm{C}$ for $2 \mathrm{~h}$. Purification by flash chromatography over aluminum oxide with $n$-hexane-EtOAc (1:0 to 9:1) gave the title compound $\mathbf{2 2} \mathbf{j}$ as colorless solid $(219.4 \mathrm{mg}, 64 \%): \mathrm{mp}$ $82{ }^{\circ} \mathrm{C}$ (from $n$-hexane); IR (neat) $\mathrm{cm}^{-1}: 1601(\mathrm{C}=\mathrm{N}), 1569(\mathrm{C}=\mathrm{N}) ;{ }^{1} \mathrm{H}-\mathrm{NMR}$ $\left(500 \mathrm{MHz} \mathrm{CDCl}_{3}\right) \delta: 1.39\left(9 \mathrm{H}, \mathrm{s}, 3 \times \mathrm{CH}_{3}\right), 1.90-1.95\left(2 \mathrm{H}, \mathrm{m}, \mathrm{CH}_{2}\right), 3.64(2 \mathrm{H}, \mathrm{t}$, $\left.J=5.4 \mathrm{~Hz}, \mathrm{CH}_{2}\right), 3.88\left(2 \mathrm{H}, \mathrm{t}, J=6.3 \mathrm{~Hz}, \mathrm{CH}_{2}\right), 7.38(1 \mathrm{H}, \mathrm{s}, \mathrm{Ar}), 7.41(1 \mathrm{H}, \mathrm{d}$, $J=8.6 \mathrm{~Hz}, \mathrm{Ar}), 8.31(1 \mathrm{H}, \mathrm{d}, J=8.6 \mathrm{~Hz}, \mathrm{Ar}) ;{ }^{13} \mathrm{C}-\mathrm{NMR}\left(100 \mathrm{MHz}, \mathrm{CDCl}_{3}\right) \delta$ : 21.8, 29.9 (3C), 45.2, 45.4, 54.3, $121.6(\mathrm{q}, J=4.0 \mathrm{~Hz}), 122.4(\mathrm{q}, J=3.6 \mathrm{~Hz})$, $123.5(\mathrm{q}, J=272.7 \mathrm{~Hz}), 129.2,130.1,130.7,132.0(\mathrm{q}, J=33.2 \mathrm{~Hz}), 136.9$, 146.9; ${ }^{19} \mathrm{~F}-\mathrm{NMR}\left(500 \mathrm{MHz}, \mathrm{CDCl}_{3}\right.$ ) $\delta$ : -63.6 ; HRMS (FAB): $\mathrm{m} / \mathrm{z}$ calcd for $\mathrm{C}_{16} \mathrm{H}_{19} \mathrm{~F}_{3} \mathrm{~N}_{3} \mathrm{~S}[\mathrm{M}+\mathrm{H}]^{+}$342.1252; found: 342.1252 .

Compound 25j. Using the general procedure as described for $\mathbf{2 5 a}$, compound 22j (68.3 mg, $0.20 \mathrm{mmol}$ ) was allowed to react for $1 \mathrm{~h}$. Purification by flash chromatography over aluminum oxide with $n$-hexane-EtOAc $(8: 2)$ gave the title compound $\mathbf{2 5} \mathbf{j}$ as colorless solid $\left(48.2 \mathrm{mg}, 84 \%\right.$ ): $\mathrm{mp} 91.5^{\circ} \mathrm{C}$ (from $\mathrm{CHCl}_{3}-n$ hexane); IR (neat) $\mathrm{cm}^{-1}: 1625(\mathrm{C}=\mathrm{N}), 1561(\mathrm{C}=\mathrm{N}) ;{ }^{1} \mathrm{H}-\mathrm{NMR}\left(500 \mathrm{MHz}, \mathrm{CDCl}_{3}\right)$ $\delta$ : 1.96-2.01 (2H, m, $\left.\mathrm{CH}_{2}\right), 3.71\left(2 \mathrm{H}, \mathrm{t}, J=5.7 \mathrm{~Hz}, \mathrm{CH}_{2}\right), 4.03(2 \mathrm{H}, \mathrm{t}$, $\left.J=6.3 \mathrm{~Hz}, \mathrm{CH}_{2}\right), 7.27(2 \mathrm{H}, \mathrm{m}, \mathrm{Ar}, \mathrm{NH}), 7.44(1 \mathrm{H}, \mathrm{dd}, J=8.3,1.4 \mathrm{~Hz}, \mathrm{Ar}), 8.35$ $(1 \mathrm{H}, \mathrm{d}, J=8.6 \mathrm{~Hz}, \mathrm{Ar}) ;{ }^{13} \mathrm{C}-\mathrm{NMR}\left(125 \mathrm{MHz}, \mathrm{CDCl}_{3}\right) \delta: 20.9,43.8,45.0,120.7$ $(\mathrm{q}, J=4.0 \mathrm{~Hz}), 122.7(\mathrm{q}, J=3.2 \mathrm{~Hz}), 123.3(\mathrm{q}, J=272.7 \mathrm{~Hz}), 129.6,129.7$, 
129.9, $132.5(\mathrm{q}, J=33.2 \mathrm{~Hz}), 145.6,152.1 ;{ }^{19} \mathrm{~F}-\mathrm{NMR}\left(500 \mathrm{MHz}, \mathrm{CDCl}_{3}\right) \delta$ : -63.8; Anal. calcd for $\mathrm{C}_{12} \mathrm{H}_{10} \mathrm{~F}_{3} \mathrm{~N}_{3} \mathrm{~S}$ : C, 50.52; H, 3.53; N, 14.73. Found: C, 50.51; $\mathrm{H}, 3.50 ; \mathrm{N}, 14.69$.

\subsubsection{Synthesis of 9-Bromo-3,4-dihydro-2H,6H- pyrimido[1,2-c][1,3]benzothiazin-6-imine (25k)}

2-(4-Bromo-2-fluorophenyl)-1,4,5,6-tetrahydropyrimidine (18k). 4-Bromo-2fluorobenzaldehyde $15 \mathrm{k}(1.02 \mathrm{~g}, 5.0 \mathrm{mmol})$ was subjected to the general procedure as described for $\mathbf{1 8 j}$ to give the title compound $\mathbf{1 8 k}$ as colorless crystals $(0.80 \mathrm{~g}, 62 \%)$ : mp $135-137{ }^{\circ} \mathrm{C}$ (from $\mathrm{CHCl}_{3}-n$-hexane); IR (neat) $\mathrm{cm}^{-1}: 1622$ $(\mathrm{C}=\mathrm{N}) ;{ }^{1} \mathrm{H}-\mathrm{NMR}\left(400 \mathrm{MHz}, \mathrm{CDCl}_{3}\right) \delta: 1.83-1.89\left(2 \mathrm{H}, \mathrm{m}, \mathrm{CH}_{2}\right), 3.49(4 \mathrm{H}, \mathrm{t}$, $\left.J=5.9 \mathrm{~Hz}, 2 \times \mathrm{CH}_{2}\right), 4.88(1 \mathrm{H}$, br s, NH$), 7.24(1 \mathrm{H}, \mathrm{dd}, J=11.2,2.0 \mathrm{~Hz}, \mathrm{Ar})$, $7.30(1 \mathrm{H}, \mathrm{dd}, J=8.5,2.0 \mathrm{~Hz}, \mathrm{Ar}), 7.71(1 \mathrm{H}, \mathrm{dd}, J=8.5,8.3 \mathrm{~Hz}, \mathrm{Ar}) ;{ }^{13} \mathrm{C}-\mathrm{NMR}$ $\left(100 \mathrm{MHz}, \mathrm{CDCl}_{3}\right) \delta: 20.6,42.3(2 \mathrm{C}), 119.5(\mathrm{~d}, J=27.3 \mathrm{~Hz}), 123.4$ (d, $J=3.3 \mathrm{~Hz}), 123.6(\mathrm{~d}, J=5.0 \mathrm{~Hz}), 127.7(\mathrm{~d}, J=3.3 \mathrm{~Hz}), 131.6(\mathrm{~d}, J=4.1 \mathrm{~Hz})$, 150.7, $159.8(\mathrm{~d}, J=251.6 \mathrm{~Hz})$; ${ }^{19} \mathrm{~F}-\mathrm{NMR}\left(500 \mathrm{MHz}, \mathrm{CDCl}_{3}\right) \delta$ : -114.7; Anal. calcd for $\mathrm{C}_{10} \mathrm{H}_{10} \mathrm{BrFN}_{2}$ : C, 46.72; H, 3.92; N, 10.90. Found: C, 46.66; H, 3.82; N, 10.87 .

9-Bromo- $N$-(tert-butyl)-3,4-dihydro-2H,6H-pyrimido[1,2-c][1,3]benzothiazin-6-imine (22k). Using the general procedure as described for 22e, compound 18k (257.1 mg, $1.00 \mathrm{mmol}$ ) was allowed to react at $\mathrm{rt}$ overnight. Purification by flash chromatography over aluminum oxide with $n$-hexane-EtOAc (1:0 to 9:1) gave the title compound $22 \mathrm{k}$ as colorless solid $(295.6 \mathrm{mg}, 84 \%)$ : $\mathrm{mp} 107-108{ }^{\circ} \mathrm{C}$ (from $n$-hexane); IR (neat) $\mathrm{cm}^{-1}: 1596(\mathrm{C}=\mathrm{N}) ;{ }^{1} \mathrm{H}-\mathrm{NMR}\left(400 \mathrm{MHz}, \mathrm{CDCl}_{3}\right) \delta$ : $1.38\left(9 \mathrm{H}, \mathrm{s}, 3 \times \mathrm{CH}_{3}\right), 1.87-1.93\left(2 \mathrm{H}, \mathrm{m}, \mathrm{CH}_{2}\right), 3.60\left(2 \mathrm{H}, \mathrm{t}, J=5.6 \mathrm{~Hz}, \mathrm{CH}_{2}\right)$, $3.85\left(2 \mathrm{H}, \mathrm{t}, J=6.1 \mathrm{~Hz}, \mathrm{CH}_{2}\right), 7.26-7.31(2 \mathrm{H}, \mathrm{m}, \mathrm{Ar}), 8.05(1 \mathrm{H}, \mathrm{d}, J=8.5 \mathrm{~Hz}$, $\mathrm{Ar}) ;{ }^{13} \mathrm{C}-\mathrm{NMR}\left(100 \mathrm{MHz}, \mathrm{CDCl}_{3}\right) \delta: 21.8,30.0$ (3C), 45.0, 45.4, 54.3, 124.4, 126.7, 126.8, 129.1, 130.1, 130.9, 137.2, 147.2; Anal. calcd for $\mathrm{C}_{15} \mathrm{H}_{18} \mathrm{BrN}_{3} \mathrm{~S}: \mathrm{C}$, 51.14; H, 5.15; N, 11.93. Found: C, 51.30; H, 5.07; N, 11.82.

Compound 25k. Using the general procedure as described for 25a, compound 22k (52.8 mg, $0.15 \mathrm{mmol})$ was allowed to react for $2 \mathrm{~h}$ with TFA (1.5 mL) and MS4A (225 mg). Purification by flash chromatography over silica gel with n-hexane-EtOAc (2:1) gave the title compound 25k as colorless solid (40.2 $\mathrm{mg}$, $91 \%$ ): mp $104-105{ }^{\circ} \mathrm{C}$ (from $\mathrm{CHCl}_{3}-n$-hexane); IR (neat) $\mathrm{cm}^{-1}: 1620(\mathrm{C}=\mathrm{N})$, $1569(\mathrm{C}=\mathrm{N}) ;{ }^{1} \mathrm{H}-\mathrm{NMR}\left(400 \mathrm{MHz}, \mathrm{CDCl}_{3}\right) \delta: 1.94-1.99\left(2 \mathrm{H}, \mathrm{m}, \mathrm{CH}_{2}\right), 3.67(2 \mathrm{H}, \mathrm{t}$, $\left.J=5.5 \mathrm{~Hz}, \mathrm{CH}_{2}\right), 4.00\left(2 \mathrm{H}, \mathrm{t}, J=6.0 \mathrm{~Hz}, \mathrm{CH}_{2}\right), 7.19-7.34(3 \mathrm{H}, \mathrm{m}, \mathrm{NH}, \mathrm{Ar})$, $8.08(1 \mathrm{H}, \mathrm{d}, J=8.8 \mathrm{~Hz}, \mathrm{Ar}) ;{ }^{13} \mathrm{C}-\mathrm{NMR}\left(100 \mathrm{MHz}, \mathrm{CDCl}_{3}\right) \delta: 20.9,43.8,44.9$, $125.0,125.6,125.9,129.5,130.4,130.7,145.8,152.4 ;$ Anal. calcd for $\mathrm{C}_{11} \mathrm{H}_{10} \mathrm{BrN}_{3} \mathrm{~S}: \mathrm{C}, 44.61 ; \mathrm{H}, 3.40 ; \mathrm{N}, 14.19$. Found: C, 44.37; H, 3.28; N, 13.93. 


\subsubsection{Synthesis of 3,4-Dihydro-9-phenyl-2H, 6H-pyrimido[1,2-c][1,3]benzothiazin-6-imine (25l)}

$N$-(tert-Butyl)-3,4-dihydro-9-phenyl-2H,6H-pyrimido[1,2-c][1,3]benzothiazin6-imine (22l). To a solution of $\mathrm{N}$-(tert-butyl)-9-bromo-3,4-dihydro- $2 \mathrm{H}, 6 \mathrm{H}$ pyrimido[1,2-c][1,3]benzothiazin-6-imine $\quad \mathbf{2 2 k} \quad(52.8 \mathrm{mg}, \quad 0.15 \mathrm{mmol})$ and phenylboronic acid $(21.9 \mathrm{mg}, 0.18 \mathrm{mmol})$ in a mixture of toluene $(1.5 \mathrm{~mL}), \mathrm{EtOH}$ $(0.9 \mathrm{~mL})$ and $1 \mathrm{M}$ aq. $\mathrm{K}_{2} \mathrm{CO}_{3}(1.5 \mathrm{~mL})$ was added $\mathrm{Pd}\left(\mathrm{PPh}_{3}\right)_{4}(6.9 \mathrm{mg}, 4 \mathrm{~mol} \%)$ and $\mathrm{PdCl}_{2}(\mathrm{dppf}) \cdot \mathrm{CH}_{2} \mathrm{Cl}_{2}(3.7 \mathrm{mg}, 3 \mathrm{~mol} \%)$. After being stirred at reflux for $1 \mathrm{~h}$, the mixture was extracted with $\mathrm{CHCl}_{3}$. The extract was dried over $\mathrm{MgSO}_{4}$ and concentrated. The residue was purified by flash chromatography over aluminum oxide with $n$-hexane-EtOAc (1:0 to 9:1) to give the title compound 22l as colorless solid (44.8 mg, $85 \%$ ): $\mathrm{mp} 122.5-124{ }^{\circ} \mathrm{C}$ (from $\mathrm{CHCl}_{3}-n$-hexane); IR (neat) $\mathrm{cm}^{-1}$ : $1592(\mathrm{C}=\mathrm{N})$; ${ }^{1} \mathrm{H}-\mathrm{NMR}\left(500 \mathrm{MHz}, \mathrm{CDCl}_{3}\right) \delta: 1.40\left(9 \mathrm{H}, \mathrm{s}, 3 \times \mathrm{CH}_{3}\right)$, 1.90-1.95 (2H, m, $\left.\mathrm{CH}_{2}\right), 3.64\left(2 \mathrm{H}, \mathrm{t}, J=5.4 \mathrm{~Hz}, \mathrm{CH}_{2}\right), 3.89(2 \mathrm{H}, \mathrm{t}, J=6.0 \mathrm{~Hz}$, $\left.\mathrm{CH}_{2}\right), 7.33-7.37(2 \mathrm{H}, \mathrm{m}, \mathrm{Ar}), 7.41-7.44(3 \mathrm{H}, \mathrm{m}, \mathrm{Ar}), 7.58(2 \mathrm{H}, \mathrm{d}, J=6.9 \mathrm{~Hz}, \mathrm{Ar})$, $8.25(1 \mathrm{H}, \mathrm{d}, J=8.6 \mathrm{~Hz}, \mathrm{Ar}) ;{ }^{13} \mathrm{C}-\mathrm{NMR}\left(125 \mathrm{MHz}, \mathrm{CDCl}_{3}\right) \delta: 21.9,30.0(3 \mathrm{C})$, 45.1, 45.4, 54.2, 122.7, 124.8, 126.5, 127.0 (2C), 128.0, 128.8 (2C), 128.9, 129.5, 138.3, 139.4, 142.9, 147.7; HRMS (FAB): $m / z$ calcd for $\mathrm{C}_{21} \mathrm{H}_{24} \mathrm{~N}_{3} \mathrm{~S}[\mathrm{M}+\mathrm{H}]^{+}$ 350.1691; found: 350.1683 .

Compound 25l. Using the general procedure as described for 25a, compound 221 (25.1 mg, $0.07 \mathrm{mmol})$ was allowed to react for $1 \mathrm{~h}$ with TFA (1.0 mL) and MS4A (105 mg). Purification by flash chromatography over aluminum oxide with $n$-hexane-EtOAc $(8: 2)$ gave the title compound 25l as pale yellow solid $(19.4 \mathrm{mg}$, $92 \%$ ): $\mathrm{mp} 122-124{ }^{\circ} \mathrm{C}$ (from $\mathrm{CHCl}_{3}-n$-hexane); IR (neat) $\mathrm{cm}^{-1}: 1619(\mathrm{C}=\mathrm{N}$ ), $1567(\mathrm{C}=\mathrm{N}) ;{ }^{1} \mathrm{H}-\mathrm{NMR}\left(500 \mathrm{MHz}, \mathrm{CDCl}_{3}\right) \delta: 1.97-2.02\left(2 \mathrm{H}, \mathrm{m}, \mathrm{CH}_{2}\right), 3.72(2 \mathrm{H}, \mathrm{t}$, $\left.J=5.4 \mathrm{~Hz}, \mathrm{CH}_{2}\right), 4.04\left(2 \mathrm{H}, \mathrm{t}, J=6.3 \mathrm{~Hz}, \mathrm{CH}_{2}\right), 7.25-7.26(1 \mathrm{H}, \mathrm{m}, \mathrm{Ar})$, 7.37-7.40 (1H, m, Ar), 7.43-7.47 (3H, m, Ar), 7.58 (2H, d, J=7.4 Hz, Ar), 8.29 $(1 \mathrm{H}, \mathrm{d}, J=8.6 \mathrm{~Hz}, \mathrm{Ar}) ;{ }^{13} \mathrm{C}-\mathrm{NMR}\left(125 \mathrm{MHz}, \mathrm{CDCl}_{3}\right) \delta: 21.1,43.8,45.0,121.8$, 121.8, 125.1, 125.5, 127.0 (2C), 128.2, 128.9 (2C), 129.4, 139.2, 143.5, 146.5, 153.4; HRMS (FAB): $m / z$ calcd for $\mathrm{C}_{17} \mathrm{H}_{16} \mathrm{~N}_{3} \mathrm{~S}[\mathrm{M}+\mathrm{H}]^{+}$294.1065; found: 294.1069 .

\subsubsection{Synthesis of 3,4-Dihydro-9-vinyl-2H, 6H-pyrimido[1,2-c][1,3]benzothiazin-6-imine (25m)}

$N$-(tert-Butyl)-3,4-dihydro-9-vinyl-2H,6H-pyrimido[1,2-c][1,3]benzothiazin-6imine (22m). Using the general procedure as described for 221, $\mathrm{N}$-(tert-butyl)-9bromo-3,4-dihydro- $2 \mathrm{H}, 6 \mathrm{H}$-pyrimido[1,2-c][1,3]benzothiazin-6-imine $22 \mathrm{k} \quad(528.4$ $\mathrm{mg}, 1.5 \mathrm{mmol}$ ) was allowed to react with vinylboronic acid pinacol ester $(0.305 \mathrm{~mL}, 1.8 \mathrm{mmol})$ for $1 \mathrm{~h}$. Purification by flash chromatography over aluminum oxide with $n$-hexane-EtOAc (1:0 to 9:1) gave the title compound $22 \mathrm{~m}$ as 
colorless solid $(455.7 \mathrm{mg},>99 \%): \mathrm{mp} 67-68{ }^{\circ} \mathrm{C}$ (from $n$-hexane); IR (neat) $\mathrm{cm}^{-1}$ : $1589(\mathrm{C}=\mathrm{N})$; ${ }^{1} \mathrm{H}-\mathrm{NMR}\left(400 \mathrm{MHz}, \mathrm{CDCl}_{3}\right) \delta: 1.39\left(9 \mathrm{H}, \mathrm{s}, 3 \times \mathrm{CH}_{3}\right)$, 1.88-1.94 (2H, m, $\left.\mathrm{CH}_{2}\right), 3.62\left(2 \mathrm{H}, \mathrm{t}, J=5.6 \mathrm{~Hz}, \mathrm{CH}_{2}\right), 3.87(2 \mathrm{H}, \mathrm{t}, J=6.1 \mathrm{~Hz}$, $\left.\mathrm{CH}_{2}\right), 5.33(1 \mathrm{H}, \mathrm{d}, J=11.0 \mathrm{~Hz}, \mathrm{CH}), 5.79(1 \mathrm{H}, \mathrm{d}, J=17.6 \mathrm{~Hz}, \mathrm{CH}), 6.64(1 \mathrm{H}$, $\mathrm{dd}, J=17.6,11.0 \mathrm{~Hz}, \mathrm{CH}), 7.12(1 \mathrm{H}, \mathrm{d}, J=1.7 \mathrm{~Hz}, \mathrm{Ar}), 7.23(1 \mathrm{H}, \mathrm{dd}, J=8.3$, $1.7 \mathrm{~Hz}, \mathrm{Ar}), 8.14(1 \mathrm{H}, \mathrm{d}, J=8.3 \mathrm{~Hz}, \mathrm{Ar}) ;{ }^{13} \mathrm{C}-\mathrm{NMR}\left(100 \mathrm{MHz}, \mathrm{CDCl}_{3}\right) \delta: 21.9$, 30.0 (3C), 45.1, 45.4, 54.1, 115.9, 122.1, 123.7, 127.0, 128.6, 129.3, 135.4, 138.3, 139.3, 147.7; HRMS (FAB): $m / z$ calcd for $\mathrm{C}_{17} \mathrm{H}_{22} \mathrm{~N}_{3} \mathrm{~S}[\mathrm{M}+\mathrm{H}]^{+} 300.1534$; found: 300.1536 .

Compound $25 \mathrm{~m}$. Using the general procedure as described for $\mathbf{2 5 a}$, compound 22m $(60.4 \mathrm{mg}, 0.2 \mathrm{mmol})$ was allowed to react for $1 \mathrm{~h}$. Purification by flash chromatography over aluminum oxide with $n$-hexane-EtOAc $(8: 2)$ gave the title compound $25 \mathrm{~m}$ as colorless solid $\left(42.1 \mathrm{mg}, 87 \%\right.$ ): $\mathrm{mp} 76-77{ }^{\circ} \mathrm{C}$ (from $\mathrm{CHCl}_{3}-n$ hexane); IR (neat) $\mathrm{cm}^{-1}: 1618(\mathrm{C}=\mathrm{N}), 1564(\mathrm{C}=\mathrm{N}) ;{ }^{1} \mathrm{H}-\mathrm{NMR}\left(400 \mathrm{MHz}, \mathrm{CDCl}_{3}\right)$ $\delta: 1.95-2.01\left(2 \mathrm{H}, \mathrm{m}, \mathrm{CH}_{2}\right), 3.69\left(2 \mathrm{H}, \mathrm{t}, J=5.4 \mathrm{~Hz}, \mathrm{CH}_{2}\right), 4.02(2 \mathrm{H}, \mathrm{t}, J=6.1 \mathrm{~Hz}$, $\left.\mathrm{CH}_{2}\right), 5.36(1 \mathrm{H}, \mathrm{d}, J=10.9 \mathrm{~Hz}, \mathrm{CH}), 5.81(1 \mathrm{H}, \mathrm{d}, J=17.7 \mathrm{~Hz}, \mathrm{CH}), 6.65(1 \mathrm{H}$, $\mathrm{dd}, J=17.7,10.9 \mathrm{~Hz}, \mathrm{CH}), 7.04(1 \mathrm{H}, \mathrm{s}, \mathrm{Ar}), 7.20(1 \mathrm{H}, \mathrm{br} \mathrm{s}, \mathrm{NH}), 7.26-7.28(1 \mathrm{H}$, $\mathrm{m}, \mathrm{Ar}), 8.17(1 \mathrm{H}, \mathrm{d}, J=8.5 \mathrm{~Hz}, \mathrm{Ar}) ;{ }^{13} \mathrm{C}-\mathrm{NMR}\left(125 \mathrm{MHz}, \mathrm{CDCl}_{3}\right) \delta: 21.0,43.8$, 44.9, 116.4, 121.1, 124.0, 125.8, 129.0 (2C), 135.2, 139.8, 146.4, 153.3; HRMS (FAB): $m / z$ calcd for $\mathrm{C}_{13} \mathrm{H}_{14} \mathrm{~N}_{3} \mathrm{~S}[\mathrm{M}+\mathrm{H}]^{+}$244.0908; found: 244.0911 .

\subsubsection{Synthesis of 3,4-Dihydro-9-styryl-2H, 6H-pyrimido[1,2-c][1,3]benzothiazin-6-imine (25n)}

\section{$N$-(tert-Butyl)-3,4-dihydro-9-styryl-2H,6H-pyrimido[1,2-c][1,3]benzothiazin-}

6-imine (22n). Using the general procedure as described for 221, $\mathrm{N}$-(tert-butyl)-9bromo-3,4-dihydro- $2 H, 6 H$-pyrimido[1,2-c][1,3]benzothiazin-6-imine

22k

$(52.8 \mathrm{mg}, 0.15 \mathrm{mmol})$ was allowed to react with styrylboronic acid pinacol ester (41.4 mg, $0.18 \mathrm{mmol}$ ) for $1 \mathrm{~h}$. Purification by flash chromatography over aluminum oxide with $n$-hexane-EtOAc (1:0 to 9:1) gave the title compound 22n as colorless solid (50.9 mg, $90 \%$ ): $\mathrm{mp} 124.5-125{ }^{\circ} \mathrm{C}$ (from $\mathrm{CHCl}_{3}-n$-hexane); IR (neat) $\mathrm{cm}^{-1}: 1590(\mathrm{C}=\mathrm{N}) ;{ }^{1} \mathrm{H}-\mathrm{NMR}\left(400 \mathrm{MHz}, \mathrm{CDCl}_{3}\right) \delta: 1.40\left(9 \mathrm{H}, \mathrm{s}, 3 \times \mathrm{CH}_{3}\right)$, 1.88-1.94 (2H, m, $\left.\mathrm{CH}_{2}\right), 3.63\left(2 \mathrm{H}, \mathrm{t}, J=5.5 \mathrm{~Hz}, \mathrm{CH}_{2}\right), 3.87(2 \mathrm{H}, \mathrm{t}, J=6.1 \mathrm{~Hz}$, $\left.\mathrm{CH}_{2}\right), 7.01(1 \mathrm{H}, \mathrm{d}, J=16.3 \mathrm{~Hz}, \mathrm{CH}), 7.14(1 \mathrm{H}, \mathrm{d}, J=16.3 \mathrm{~Hz}, \mathrm{CH}), 7.22(1 \mathrm{H}, \mathrm{d}$, $J=1.7 \mathrm{~Hz}, \mathrm{Ar}), 7.27-7.38(4 \mathrm{H}, \mathrm{m}, \mathrm{Ar}), 7.50(2 \mathrm{H}, \mathrm{d}, J=7.3 \mathrm{~Hz}, \mathrm{Ar}), 8.17(1 \mathrm{H}, \mathrm{d}$, $J=8.3 \mathrm{~Hz}, \mathrm{Ar}) ;{ }^{13} \mathrm{C}-\mathrm{NMR}\left(100 \mathrm{MHz}, \mathrm{CDCl}_{3}\right) \delta: 21.9,30.0$ (3C), 45.1, 45.4, 54.2, 122.2, 124.0, 126.6, 126.7 (2C), 127.0, 128.1, 128.7 (2C), 128.8, 129.4, 130.7, 136.8, 138.3, 139.2, 147.7; HRMS (FAB): $\mathrm{m} / \mathrm{z}$ calcd for $\mathrm{C}_{23} \mathrm{H}_{26} \mathrm{~N}_{3} \mathrm{~S}$ $[\mathrm{M}+\mathrm{H}]^{+}$376.1847; found: 376.1845 .

Compound 25n. Using the general procedure as described for 25a, compound 22n (31.7 mg, $0.084 \mathrm{mmol}$ ) was allowed to react for $2 \mathrm{~h}$. Purification by flash chromatography over aluminum oxide with $n$-hexane-EtOAc $(7: 3)$ gave the title 
compound 25n as colorless solid $\left(20.2 \mathrm{mg}, 75 \%\right.$ ): $\mathrm{mp} 111-113{ }^{\circ} \mathrm{C}$ (from $\mathrm{CHCl}_{3}-$ $n$-hexane); IR (neat) $\mathrm{cm}^{-1}$ : $1618(\mathrm{C}=\mathrm{N}), 1567(\mathrm{C}=\mathrm{N}) ;{ }^{1} \mathrm{H}-\mathrm{NMR}(400 \mathrm{MHz}$, $\left.\mathrm{CDCl}_{3}\right) \delta: 1.94-2.00\left(2 \mathrm{H}, \mathrm{m}, \mathrm{CH}_{2}\right), 3.69\left(2 \mathrm{H}, \mathrm{t}, J=5.6 \mathrm{~Hz}, \mathrm{CH}_{2}\right), 4.02(2 \mathrm{H}, \mathrm{t}$, $\left.J=6.2 \mathrm{~Hz}, \mathrm{CH}_{2}\right), 7.00(1 \mathrm{H}, \mathrm{d}, J=16.3 \mathrm{~Hz}, \mathrm{CH}), 7.12-7.16(2 \mathrm{H}, \mathrm{m}, \mathrm{CH}, \mathrm{Ar})$, $7.20(1 \mathrm{H}$, br s, NH), 7.26-7.30 (1H, m, Ar), 7.34-7.38 (3H, m, Ar), $7.50(2 \mathrm{H}, \mathrm{d}$, $J=7.6 \mathrm{~Hz}, \mathrm{Ar}), 8.20(1 \mathrm{H}, \mathrm{d}, J=8.5 \mathrm{~Hz}, \mathrm{Ar}) ;{ }^{13} \mathrm{C}-\mathrm{NMR}\left(100 \mathrm{MHz}, \mathrm{CDCl}_{3}\right) \delta$ : 21.0, 43.8, 45.0, 121.2, 124.2, 125.5, 126.6, 126.7 (2C), 128.2, 128.7 (2C), 129.1, 129.2, 131.1, 136.6, 139.7, 146.4, 153.3; Anal. calcd for $\mathrm{C}_{19} \mathrm{H}_{17} \mathrm{~N}_{3} \mathrm{~S}: \mathrm{C}, 71.44 ; \mathrm{H}$, 5.36; N, 13.15. Found: C, 71.17; H, 5.24; N, 13.07.

\subsubsection{Synthesis of 3,4-Dihydro-9-pentenyl-2H, 6H-pyrimido[1,2-c][1,3]benzothiazin-6-imine (25o)}

$N$-(tert-Butyl)-3,4-dihydro-9-pentenyl-2H,6H-pyrimido[1,2-c][1,3]benzothiazin-6-imine (22o). Using the general procedure as described for $\mathbf{2 2 1}, N$-(tert-butyl)9-bromo-3,4-dihydro-2H,6 $H$-pyrimido[1,2-c][1,3]benzothiazin-6-imine $\quad 22 k$ $(52.8 \mathrm{mg}, 0.15 \mathrm{mmol})$ was allowed to react with pentenylboronic acid pinacol ester (35.2 $\mathrm{mg}, 0.18 \mathrm{mmol}$ ) for $1 \mathrm{~h}$. Purification by flash chromatography over aluminum oxide with $n$-hexane-EtOAc (1:0 to 9:1) gave the title compound 22o as a colorless oil (44.2 mg, $86 \%)$ : IR (neat) $\mathrm{cm}^{-1}: 1590(\mathrm{C}=\mathrm{N}) ;{ }^{1} \mathrm{H}-\mathrm{NMR}(400 \mathrm{MHz}$, $\left.\mathrm{CDCl}_{3}\right) \delta ; 0.95\left(\mathrm{t}, J=7.4 \mathrm{~Hz}, 3 \mathrm{H}, \mathrm{CH}_{3}\right), 1.38\left(9 \mathrm{H}, \mathrm{s}, 3 \times \mathrm{CH}_{3}\right), 1.46-1.54(2 \mathrm{H}, \mathrm{m}$, $\left.\mathrm{CH}_{2}\right), 1.87-1.93\left(2 \mathrm{H}, \mathrm{m}, \mathrm{CH}_{2}\right), 2.16-2.21\left(2 \mathrm{H}, \mathrm{m}, \mathrm{CH}_{2}\right), 3.61(2 \mathrm{H}, \mathrm{t}, J=5.6 \mathrm{~Hz}$, $\left.\mathrm{CH}_{2}\right), 3.86\left(2 \mathrm{H}, \mathrm{t}, J=6.2 \mathrm{~Hz}, \mathrm{CH}_{2}\right), 6.29-6.30(2 \mathrm{H}, \mathrm{m}, 2 \times \mathrm{CH}), 7.05(1 \mathrm{H}, \mathrm{d}$, $J=1.7 \mathrm{~Hz}, \mathrm{Ar}), 7.17(1 \mathrm{H}, \mathrm{dd}, J=8.3,1.7 \mathrm{~Hz}, \mathrm{Ar}), 8.10(1 \mathrm{H}, \mathrm{d}, J=8.3 \mathrm{~Hz}, \mathrm{Ar})$; ${ }^{13} \mathrm{C}-\mathrm{NMR}\left(100 \mathrm{MHz}, \mathrm{CDCl}_{3}\right) \delta: 13.7,21.9,22.3,30.0$ (3C), 35.1, 45.1, 45.4, 54.1, 121.6, 123.6, 126.0, 128.5, 128.6, 129.1 133.4, 138.5, 139.8, 147.8; HRMS (FAB): $\mathrm{m} / \mathrm{z}$ calcd for $\mathrm{C}_{20} \mathrm{H}_{28} \mathrm{~N}_{3} \mathrm{~S}[\mathrm{M}+\mathrm{H}]^{+} 342.2004$; found: 342.2007 .

Compound 25o. Using the general procedure as described for $\mathbf{2 5 a}$, compound $220(40.0 \mathrm{mg}, 0.12 \mathrm{mmol}$ ) was allowed to react for $2 \mathrm{~h}$. Purification by flash chromatography over aluminum oxide with $n$-hexane-EtOAc (8:2) gave the title compound 250 as a colorless oil $(31.9 \mathrm{mg}, 95 \%)$ : IR (neat) $\mathrm{cm}^{-1}: 1619(\mathrm{C}=\mathrm{N})$, $1568(\mathrm{C}=\mathrm{N}) ;{ }^{1} \mathrm{H}-\mathrm{NMR}\left(400 \mathrm{MHz}, \mathrm{CDCl}_{3}\right) \delta ; 0.95\left(3 \mathrm{H}, \mathrm{t}, J=7.4 \mathrm{~Hz}, \mathrm{CH}_{3}\right)$, 1.45-1.54 (2H, m, $\left.\mathrm{CH}_{2}\right), 1.93-1.99\left(2 \mathrm{H}, \mathrm{m}, \mathrm{CH}_{2}\right), 2.17-2.22\left(2 \mathrm{H}, \mathrm{m}, \mathrm{CH}_{2}\right), 3.68$ $\left(2 \mathrm{H}, \mathrm{t}, J=5.5 \mathrm{~Hz}, \mathrm{CH}_{2}\right), 4.01\left(2 \mathrm{H}, \mathrm{t}, J=6.2 \mathrm{~Hz}, \mathrm{CH}_{2}\right), 6.29-6.31(2 \mathrm{H}, \mathrm{m}$, $2 \times \mathrm{CH}), 6.96(1 \mathrm{H}, \mathrm{d}, J=1.7 \mathrm{~Hz}, \mathrm{Ar}), 7.16(1 \mathrm{H}$, br s, NH$), 7.19(1 \mathrm{H}, \mathrm{dd}, J=8.5$, $1.7 \mathrm{~Hz}, \mathrm{Ar}), 8.13(1 \mathrm{H}, \mathrm{d}, J=8.5 \mathrm{~Hz}, \mathrm{Ar}) ;{ }^{13} \mathrm{C}-\mathrm{NMR}\left(100 \mathrm{MHz}, \mathrm{CDCl}_{3}\right) \delta: 13.7$, 21.0, 22.3, 35.1, 43.8, 44.9, 120.6, 123.8, 124.9, 128.3, 128.9, 129.0, 133.9, 140.3, 146.5, 153.5; HRMS (FAB): $m / z$ calcd for $\mathrm{C}_{16} \mathrm{H}_{20} \mathrm{~N}_{3} \mathrm{~S}[\mathrm{M}+\mathrm{H}]^{+}$286.1378; found:286.1376. 


\subsubsection{Synthesis of 9-Azido-3,4-dihydro-2H, 6H-pyrimido[1,2-c][1,3]benzothiazin-6-imine (25p)}

9-Azido- $N$-(tert-butyl)-3,4-dihydro-2H,6H-pyrimido[1,2-c $][1,3]$ benzothiazin-6imine (22p). To a solution of 9-amino- $\mathrm{N}$-(tert-butyl)-3,4-dihydro- $2 \mathrm{H}, 6 \mathrm{H}$-pyrimido[1,2-c][1,3] benzothiazin-6-imine $28(100.9 \mathrm{mg}, 0.35 \mathrm{mmol})$ in $\mathrm{AcOH}(2 \mathrm{~mL})$ and $\mathrm{H}_{2} \mathrm{O}(1 \mathrm{~mL})$ was added $\mathrm{NaNO}_{2}(33.8 \mathrm{mg}, 0.49 \mathrm{mmol})$ at $0{ }^{\circ} \mathrm{C}$, and the stirring was continued for $1 \mathrm{~h} . \mathrm{NaN}_{3}(34.1 \mathrm{mg}, 0.53 \mathrm{mmol})$ was added to the reaction mixture, and stirring was continued for $30 \mathrm{~min}$ at rt. Reaction mixture was neutralized with $\mathrm{K}_{2} \mathrm{CO}_{3}$, and the whole was extracted with $\mathrm{CHCl}_{3}$, and dried over $\mathrm{MgSO}_{4}$. After concentration, the residue was purified by flash chromatography over aluminum oxide with $n$-hexane-EtOAc (9:1) to give the title compound 22p as pale yellow solid $(77.3 \mathrm{mg}, 70 \%)$ : mp 79-80 ${ }^{\circ} \mathrm{C}$ (from $n$-hexane); IR (neat) $\mathrm{cm}^{-1}$ : $2104\left(\mathrm{~N}_{3}\right), 1592(\mathrm{C}=\mathrm{N}) ;{ }^{1} \mathrm{H}-\mathrm{NMR}\left(400 \mathrm{MHz}, \mathrm{CDCl}_{3}\right) \delta: 1.38(9 \mathrm{H}, \mathrm{s}$, $\left.3 \times \mathrm{CH}_{3}\right), 1.88-1.94\left(2 \mathrm{H}, \mathrm{m}, \mathrm{CH}_{2}\right), 3.60\left(2 \mathrm{H}, \mathrm{t}, J=5.6 \mathrm{~Hz}, \mathrm{CH}_{2}\right), 3.86(2 \mathrm{H}, \mathrm{t}$, $\left.J=6.2 \mathrm{~Hz}, \mathrm{CH}_{2}\right), 6.74(1 \mathrm{H}, \mathrm{d}, J=2.3 \mathrm{~Hz}, \mathrm{Ar}), 6.84(1 \mathrm{H}, \mathrm{dd}, J=8.5,2.3 \mathrm{~Hz}$, $\mathrm{Ar}), 8.19(1 \mathrm{H}, \mathrm{d}, J=8.5 \mathrm{~Hz}, \mathrm{Ar}) ;{ }^{13} \mathrm{C}-\mathrm{NMR}\left(100 \mathrm{MHz}, \mathrm{CDCl}_{3}\right) \delta: 21.8,30.0$ (3C), 45.0, 45.4, 54.2, 114.2, 116.8, 124.5, 130.3, 130.9, 137.4, 142.0, 147.1; HRMS (FAB): $m / z$ calcd for $\mathrm{C}_{15} \mathrm{H}_{19} \mathrm{~N}_{6} \mathrm{~S}[\mathrm{M}+\mathrm{H}]^{+}$315.1392; found: 315.1398 .

Compound 25p. Using the general procedure as described for 25a, compound 22p $(77.3 \mathrm{mg}, 0.25 \mathrm{mmol})$ was allowed to react for $2 \mathrm{~h}$ with TFA $(3.5 \mathrm{~mL})$ and MS4 $\AA$ (525 mg). Purification by recrystallization from $\mathrm{MeOH}-\mathrm{Et}_{2} \mathrm{O}$ gave the title compound $25 \mathrm{p}$ as pale yellow crystals $(27.0 \mathrm{mg}, 42 \%)$ : $\mathrm{mp} 120-121{ }^{\circ} \mathrm{C}$; IR (neat) $\mathrm{cm}^{-1}$ : $2107\left(\mathrm{~N}_{3}\right), 1615(\mathrm{C}=\mathrm{N}), 1569(\mathrm{C}=\mathrm{N}) ;{ }^{1} \mathrm{H}-\mathrm{NMR}\left(400 \mathrm{MHz}, \mathrm{DMSO}-d_{6}\right) \delta$ : $1.82-1.88\left(2 \mathrm{H}, \mathrm{m}, \mathrm{CH}_{2}\right), 3.56\left(2 \mathrm{H}, \mathrm{t}, J=5.5 \mathrm{~Hz}, \mathrm{CH}_{2}\right), 3.89(2 \mathrm{H}, \mathrm{t}, J=5.4 \mathrm{~Hz}$, $\left.\mathrm{CH}_{2}\right), 6.97(1 \mathrm{H}, \mathrm{dd}, J=8.8,2.4 \mathrm{~Hz}, \mathrm{Ar}), 7.03(1 \mathrm{H}, \mathrm{d}, J=2.4 \mathrm{~Hz}, \mathrm{Ar}), 8.17(1 \mathrm{H}$, $\mathrm{d}, J=8.8 \mathrm{~Hz}, \mathrm{Ar}), 8.76(1 \mathrm{H}, \mathrm{s}, \mathrm{NH}) ;{ }^{13} \mathrm{C}-\mathrm{NMR}\left(100 \mathrm{MHz}, \mathrm{DMSO}-d_{6}\right) \delta: 20.6$, 43.1, 44.2, 113.7, 117.0, 122.6, 130.2, 130.8, 141.9, 144.7, 150.0; Anal. calcd for $\mathrm{C}_{11} \mathrm{H}_{10} \mathrm{~N}_{6} \mathrm{~S}$ : C, 51.15; H, 3.90; N, 32.54. Found: C, 51.07; H, 3.88; N, 32.28.

\subsubsection{Synthesis of 9-(4-Benzoylphenyl)-3,4-dihydro-2H, 6H-pyrimido[1,2-c][1,3]benzothiazin-6-imine (25q)}

9-(4-Benzoylphenyl)- $N$-(tert-butyl)-3,4-dihydro-2H, $\quad 6 H$-pyrimido[1,2-c $][1,3]$ benzothiazin-6-imine (22q). Using the general procedure as described for 221 , $\mathrm{N}$-(tert-butyl)-9-bromo-3,4-dihydro- $2 \mathrm{H}, 6 \mathrm{H}$-pyrimido[1,2-c][1,3] benzothiazin-6imine 22k (52.8 mg, $0.15 \mathrm{mmol})$ was allowed to react with 4-benzoylphenylboronic acid $(40.7 \mathrm{mg}, 0.18 \mathrm{mmol}$ ) for $1 \mathrm{~h}$. Purification by flash chromatography over aluminum oxide with $n$-hexane-EtOAc (8:2) gave the title compound $\mathbf{2 2 q}$ as colorless solid (55.6 mg, $82 \%$ ): $\mathrm{mp} 187-189{ }^{\circ} \mathrm{C}$ (from $\mathrm{CHCl}_{3}-n$-hexane); IR (neat) $\mathrm{cm}^{-1}: 1656(\mathrm{C}=\mathrm{O}), 1593(\mathrm{C}=\mathrm{N}) ;{ }^{1} \mathrm{H}-\mathrm{NMR}\left(400 \mathrm{MHz}, \mathrm{CDCl}_{3}\right) \delta: 1.41(9 \mathrm{H}$, s, $\left.3 \times \mathrm{CH}_{3}\right), 1.91-1.97\left(2 \mathrm{H}, \mathrm{m}, \mathrm{CH}_{2}\right), 3.65\left(2 \mathrm{H}, \mathrm{t}, J=5.5 \mathrm{~Hz}, \mathrm{CH}_{2}\right), 3.90(2 \mathrm{H}, \mathrm{t}$, $\left.J=6.1 \mathrm{~Hz}, \mathrm{CH}_{2}\right), 7.39(1 \mathrm{H}, \mathrm{d}, J=1.7 \mathrm{~Hz}, \mathrm{Ar}), 7.46-7.53(3 \mathrm{H}, \mathrm{m}, \mathrm{Ar}), 7.60(1 \mathrm{H}$, 
$\mathrm{t}, J=7.4 \mathrm{~Hz}, \mathrm{Ar}), 7.70(2 \mathrm{H}, \mathrm{d}, J=8.0 \mathrm{~Hz}, \mathrm{Ar}), 7.82(2 \mathrm{H}, \mathrm{d}, J=7.3 \mathrm{~Hz}, \mathrm{Ar})$, $7.88(2 \mathrm{H}, \mathrm{d}, J=8.0 \mathrm{~Hz}, \mathrm{Ar}), 8.30(1 \mathrm{H}, \mathrm{d}, J=8.3 \mathrm{~Hz}, \mathrm{Ar}) ;{ }^{13} \mathrm{C}-\mathrm{NMR}(100 \mathrm{MHz}$, $\left.\mathrm{CDCl}_{3}\right) \delta: 21.9,30.0$ (3C), 45.2, 45.4, 54.2, 123.0, 124.8, 126.9 (2C), 127.4, 128.3 (2C), 129.1, 129.9, 130.0 (2C), 130.7 (2C), 132.5, 136.9, 137.6, 137.9, 141.7, 143.3, 147.6, 196.1; HRMS (FAB): $m / z$ calcd for $\mathrm{C}_{28} \mathrm{H}_{28} \mathrm{~N}_{3} \mathrm{OS}[\mathrm{M}+\mathrm{H}]^{+}$ 454.1953; found: 454.1954.

Compound 25q. Using the general procedure as described for 25a, compound 22q $(30.4 \mathrm{mg}, 0.067 \mathrm{mmol})$ was allowed to react for $1 \mathrm{~h}$ with TFA $(1.0 \mathrm{~mL})$ and MS4 A (150 mg). Purification by flash chromatography over aluminum oxide with n-hexane-EtOAc (7:3) gave the title compound 25q as colorless solid (16.7 $\mathrm{mg}$, $63 \%$ ): $\mathrm{mp} 155-156{ }^{\circ} \mathrm{C}$ (from $\mathrm{CHCl}_{3}-n$-hexane); IR (neat) $\mathrm{cm}^{-1}: 1655(\mathrm{C}=\mathrm{O})$, $1619(\mathrm{C}=\mathrm{N}), 1561(\mathrm{C}=\mathrm{N}) ;{ }^{1} \mathrm{H}-\mathrm{NMR}\left(400 \mathrm{MHz}, \mathrm{CDCl}_{3}\right) \delta: 1.97-2.03(2 \mathrm{H}, \mathrm{m}$, $\left.\mathrm{CH}_{2}\right), 3.72\left(2 \mathrm{H}, \mathrm{t}, J=5.5 \mathrm{~Hz}, \mathrm{CH}_{2}\right), 4.05\left(2 \mathrm{H}, \mathrm{t}, J=6.1 \mathrm{~Hz}, \mathrm{CH}_{2}\right), 7.30(1 \mathrm{H}, \mathrm{d}$, $J=1.7 \mathrm{~Hz}, \mathrm{Ar}), 7.48-7.52(3 \mathrm{H}, \mathrm{m}, \mathrm{Ar}), 7.59-7.63(1 \mathrm{H}, \mathrm{m}, \mathrm{Ar}), 7.68(2 \mathrm{H}$, $\mathrm{d}, J=8.3 \mathrm{~Hz}, \mathrm{Ar}), 7.81-7.83(2 \mathrm{H}, \mathrm{m}, \mathrm{Ar}), 7.89(2 \mathrm{H}, \mathrm{d}, J=8.3 \mathrm{~Hz}, \mathrm{Ar}), 8.32(1 \mathrm{H}$, $\mathrm{d}, J=8.5 \mathrm{~Hz}, \mathrm{Ar}) ;{ }^{13} \mathrm{C}-\mathrm{NMR}\left(100 \mathrm{MHz}, \mathrm{CDCl}_{3}\right) \delta: 21.0,43.8,45.0,122.0,125.1$, 126.2, 126.9 (2C), 128.3 (2C), 129.5, 129.6, 130.0 (2C), 130.7 (2C), 132.5, 137.1, $137.5,142.2,143.0,146.3,153.0,196.0 ;$ HRMS (FAB): $\mathrm{m} / z$ calcd for $\mathrm{C}_{20} \mathrm{H}_{18} \mathrm{~N}_{3} \mathrm{OS}[\mathrm{M}+\mathrm{H}]^{+}$398.1327; found: 398.1333 .

\subsubsection{Synthesis of 10-(N,N-Dimethylamino)-3,4-dihydro- 2H,6H-pyrimido[1,2-c][1,3]benzothiazin-6-imine (26a)}

$N$-(tert-Butyl)-10-( $N, N$-dimethylamino)-3,4-dihydro-2H,6H-pyrimido[1,2-c $][1$, 3]benzothiazin-6-imine (23a). To a mixture of 10-bromo- $N$-(tert-butyl)-3,4dihydro-2H,6H-pyrimido[1,2-c][1,3]benzothiazin-6-imine $\quad \mathbf{2 3 k} \quad(600.2 \mathrm{mg}$, $1.70 \mathrm{mmol})$ and $\mathrm{Pd}\left(\mathrm{P} t-\mathrm{Bu}_{3}\right)_{2} \quad(174.2 \mathrm{mg}, \quad 0.341 \mathrm{mmol})$ and $\mathrm{KO} t-\mathrm{Bu}$ $(573.3 \mathrm{mg}, 5.11 \mathrm{mmol})$ in toluene $(1.7 \mathrm{~mL})$ was added $2.0 \mathrm{M} \mathrm{Me} 2 \mathrm{NH}$ in $\mathrm{THF}$ $(2.55 \mathrm{~mL}, 5.11 \mathrm{mmol})$. The reaction was heated using a microwave reactor (standard mode) for $10 \mathrm{~min}$ at $170{ }^{\circ} \mathrm{C}$. The whole was extracted with EtOAc. The extract was washed with brine, and dried over $\mathrm{Na}_{2} \mathrm{SO}_{4}$. After concentration, the residue was purified by flash chromatography over silica gel with $n$-hexane-EtOAc (6:4 to 5:5) to give the title compound 23a as pale yellow solid (363.3 $\mathrm{mg}$, $67.4 \%$ ): $\mathrm{mp} 86.1^{\circ} \mathrm{C}$; IR (neat) $\mathrm{cm}^{-1}: 1583(\mathrm{C}=\mathrm{N}) ;{ }^{1} \mathrm{H}-\mathrm{NMR}\left(300 \mathrm{MHz}, \mathrm{CDCl}_{3}\right)$ $\delta: 1.38\left(9 \mathrm{H}, \mathrm{s}, 3 \times \mathrm{CH}_{3}\right), 1.86-1.94\left(2 \mathrm{H}, \mathrm{m}, \mathrm{CH}_{2}\right), 2.97\left(6 \mathrm{H}, \mathrm{s}, 2 \times \mathrm{CH}_{3}\right), 3.61$ $\left(2 \mathrm{H}, \mathrm{t}, J=5.3 \mathrm{~Hz}, \mathrm{CH}_{2}\right), 3.86\left(2 \mathrm{H}, \mathrm{t}, J=6.3 \mathrm{~Hz}, \mathrm{CH}_{2}\right), 6.78(1 \mathrm{H}, \mathrm{dd}, J=9.0$, $3.0 \mathrm{~Hz}, \mathrm{Ar}), 6.98(1 \mathrm{H}, \mathrm{d}, J=8.4 \mathrm{~Hz}, \mathrm{Ar}), 7.56(1 \mathrm{H}, \mathrm{d}, J=2.4 \mathrm{~Hz}, \mathrm{Ar}) ;{ }^{13} \mathrm{C}-\mathrm{NMR}$ $\left(75 \mathrm{MHz}, \mathrm{CDCl}_{3}\right) \delta: 22.0,29.9$ (3C), 40.8 (2C), 45.1, 45.5, 54.0, 111.6, 115.4, 115.8, 125.3, 128.7, 139.7, 148.8, 149.3; HRMS (FAB): $m / z$ calcd for $\mathrm{C}_{17} \mathrm{H}_{25} \mathrm{~N}_{4} \mathrm{~S}$ $[\mathrm{M}+\mathrm{H}]^{+}$317.1800; found: 317.1796. 
Compound 26a. TFA $(0.63 \mathrm{~mL})$ was added to a mixture of 23a (20 mg, $0.063 \mathrm{mmol})$ and MS4 $\mathrm{A}(110 \mathrm{mg}$, powder, activated by heating with Bunsen burner) in small amount of $\mathrm{CHCl}_{3}$. After being stirred under reflux for $40 \mathrm{~min}$, the mixture was added dropwise to $\mathrm{Et}_{3} \mathrm{~N}$ at $0{ }^{\circ} \mathrm{C}$ to adjust $\mathrm{pH}$ to $8-9$. The whole was extracted with EtOAc. The extract was washed with sat. $\mathrm{NaHCO}_{3}$, brine, and dried over $\mathrm{Na}_{2} \mathrm{SO}_{4}$. After concentration, the residue was purified by preparative TLC over aluminum oxide with $n$-hexane-EtOAc (7:3) to give the title compound 26a as yellow solid (11.4 mg, $68.3 \%)$ : mp $134.5^{\circ} \mathrm{C}$; IR (neat) $\mathrm{cm}^{-1}$ : $1617(\mathrm{C}=\mathrm{N})$, $1552(\mathrm{C}=\mathrm{N}) ;{ }^{1} \mathrm{H}-\mathrm{NMR}\left(300 \mathrm{MHz}, \mathrm{CDCl}_{3}\right) \delta: 1.92-2.00\left(2 \mathrm{H}, \mathrm{m}, \mathrm{CH}_{2}\right), 2.97(6 \mathrm{H}, \mathrm{s}$, $\left.2 \times \mathrm{CH}_{3}\right), 3.68\left(2 \mathrm{H}, \mathrm{t}, J=5.7 \mathrm{~Hz}, \mathrm{CH}_{2}\right), 4.01\left(2 \mathrm{H}, \mathrm{t}, J=6.3 \mathrm{~Hz}, \mathrm{CH}_{2}\right), 6.79$ $(1 \mathrm{H}, \mathrm{dd}, J=8.7,3.3 \mathrm{~Hz}, \mathrm{Ar}), 6.89(1 \mathrm{H}, \mathrm{d}, J=8.7 \mathrm{~Hz}, \mathrm{Ar}), 7.08(1 \mathrm{H}, \mathrm{br} \mathrm{s}, \mathrm{NH})$, $7.58(1 \mathrm{H}, \mathrm{d}, J=2.7 \mathrm{~Hz}, \mathrm{Ar}) ;{ }^{13} \mathrm{C}-\mathrm{NMR}\left(75 \mathrm{MHz}, \mathrm{CDCl}_{3}\right) \delta: 20.8,40.6(2 \mathrm{C})$, 44.1, 44.3, 111.8, 114.6, 116.3, 124.6, 126.3, 148.7, 149.4, 154.4; Anal. calcd for $\mathrm{C}_{13} \mathrm{H}_{16} \mathrm{~N}_{4} \mathrm{~S}$ : C, 59.97; H, 6.19; N, 21.52. Found: C, 59.91; H, 6.19; N, 21.41.

\subsubsection{Synthesis of 3,4-Dihydro-10-nitro-2H, 6H-pyrimido[1,2-c][1,3]benzothiazin-6-imine (26e)}

$N$-(tert-Butyl)-3,4-dihydro-10-nitro-2H,6H-pyrimido[1,2-c][1,3]benzothiazin6-imine (23e). To a mixture of 2-(2-bromo-5-nitrophenyl)-1,4,5,6-tetrahydropyrimidine 19e $(50 \mathrm{mg}, 0.209 \mathrm{mmol})$ in DMAc $(0.70 \mathrm{~mL})$ were added tert-butylisothiocyanate $(0.053 \mathrm{~mL}, 0.418 \mathrm{mmol})$ and $\mathrm{KO} t$-Bu $(46.9 \mathrm{mg}, 0.418 \mathrm{mmol})$ at $0{ }^{\circ} \mathrm{C}$ under an $\mathrm{N}_{2}$ atmosphere. After being stirred at $0{ }^{\circ} \mathrm{C}$ for $1 \mathrm{~h}$, sat. $\mathrm{NH}_{4} \mathrm{Cl}$ was added. The whole was extracted with EtOAc. The extract was washed with brine, and dried over $\mathrm{Na}_{2} \mathrm{SO}_{4}$. After concentration, the residue was purified by flash chromatography over silica gel with $n$-hexane-EtOAc (1:0 to 9:1) to give the title compound $23 \mathrm{e}$ as pale yellow solid $(39.1 \mathrm{mg}, 58.9 \%)$ : $\mathrm{mp} 123.8{ }^{\circ} \mathrm{C}$; IR (neat) $\mathrm{cm}^{-1}: 1593\left(\mathrm{NO}_{2}\right), 1520\left(\mathrm{NO}_{2}\right) ;{ }^{1} \mathrm{H}-\mathrm{NMR}\left(300 \mathrm{MHz}, \mathrm{CDCl}_{3}\right) \delta: 1.40(\mathrm{~s}, 9 \mathrm{H}$, $\left.3 \times \mathrm{CH}_{3}\right), 1.90-1.98\left(2 \mathrm{H}, \mathrm{m}, \mathrm{CH}_{2}\right), 3.67\left(2 \mathrm{H}, \mathrm{t}, J=5.6 \mathrm{~Hz}, \mathrm{CH}_{2}\right), 3.89(2 \mathrm{H}, \mathrm{t}$, $\left.J=6.3 \mathrm{~Hz}, \mathrm{CH}_{2}\right), 7.23(1 \mathrm{H}, \mathrm{m}, \mathrm{Ar}), 8.13(1 \mathrm{H}, \mathrm{dd}, J=8.7,2.7 \mathrm{~Hz}, \mathrm{Ar}), 9.11(1 \mathrm{H}$, $\mathrm{d}, J=2.7 \mathrm{~Hz}, \mathrm{Ar}) ;{ }^{13} \mathrm{C}-\mathrm{NMR}\left(75 \mathrm{MHz}, \mathrm{CDCl}_{3}\right) \delta: 21.7,30.0$ (3C), 45.1, 45.5, 54.5, 124.1, 124.3, 125.3, 128.5, 135.6, 137.1, 145.8, 146.1; HRMS (FAB): $\mathrm{m} / \mathrm{z}$ calcd for $\mathrm{C}_{15} \mathrm{H}_{19} \mathrm{~N}_{4} \mathrm{O}_{2} \mathrm{~S}[\mathrm{M}+\mathrm{H}]^{+}$319.1229; found: 319.1232 .

Compound 26e. TFA $(3.2 \mathrm{~mL})$ was added to compound $23 \mathbf{e}(100 \mathrm{mg}$, $0.314 \mathrm{mmol}$ ). After being stirred under reflux for $1.5 \mathrm{~h}$, the mixture was added dropwise to $\mathrm{Et}_{3} \mathrm{~N}$ at $0{ }^{\circ} \mathrm{C}$ to adjust $\mathrm{pH}$ to $8-9$. The whole was extracted with EtOAc. The extract was washed with sat. $\mathrm{NaHCO}_{3}$, brine, and dried over $\mathrm{Na}_{2} \mathrm{SO}_{4}$. After concentration, the residue was purified by preparative TLC over aluminum oxide with $n$-hexane-EtOAc (7:3) to give the title compound 26e as orange solid (15.9 mg, $19.3 \%): \mathrm{mp} 167.9{ }^{\circ} \mathrm{C}$; IR (neat) $\mathrm{cm}^{-1}: 1614(\mathrm{C}=\mathrm{N}), 1576\left(\mathrm{NO}_{2}\right), 1557$ $(\mathrm{C}=\mathrm{N}), 1519\left(\mathrm{NO}_{2}\right) ;{ }^{1} \mathrm{H}-\mathrm{NMR}\left(300 \mathrm{MHz}, \mathrm{CDCl}_{3}\right) \delta: 1.97-2.04\left(2 \mathrm{H}, \mathrm{m}, \mathrm{CH}_{2}\right), 3.74$ $\left(2 \mathrm{H}, \mathrm{t}, J=5.6 \mathrm{~Hz}, \mathrm{CH}_{2}\right), 4.05\left(2 \mathrm{H}, \mathrm{t}, J=6.2 \mathrm{~Hz}, \mathrm{CH}_{2}\right), 7.19(1 \mathrm{H}, \mathrm{d}, J=9.0 \mathrm{~Hz}$, 
Ar), $7.38(1 \mathrm{H}$, br s, NH), $8.17(1 \mathrm{H}, \mathrm{dd}, J=8.7,2.7 \mathrm{~Hz}, \mathrm{Ar}), 9.13(1 \mathrm{H}, \mathrm{d}$, $J=2.7 \mathrm{~Hz}, \mathrm{Ar}) ;{ }^{13} \mathrm{C}-\mathrm{NMR}\left(75 \mathrm{MHz}, \mathrm{CDCl}_{3}\right) \delta: 20.8,44.1,44.9,124.5$ (2C), 124.8, 127.6, 136.7, 144.5, 146.3, 151.0; HRMS (FAB): $m / z$ calcd for $\mathrm{C}_{11} \mathrm{H}_{11} \mathrm{~N}_{4} \mathrm{O}_{2} \mathrm{~S}[\mathrm{M}+\mathrm{H}]^{+} 263.0603$; found: 263.0606 . The purity of the compound was $75 \%$ by HPLC.

\subsubsection{Synthesis of 3,4-Dihydro-10-methoxy-2H, 6H-pyrimido[1,2-c][1,3]benzothiazin-6-imine (26f)}

\section{$N$-(tert-Butyl)-3,4-dihydro-10-methoxy-2H,6H-pyrimido[1,2-c][1,3]benzothia-} zin-6-imine (23f). To a mixture of $\mathrm{N}$-(tert-butyl)-9-bromo-3,4-dihydro- $\mathrm{H}, 6 \mathrm{H}$ pyrimido[1,2-c][1,3]benzothiazin-6-imine $22 \mathbf{k}(500.3 \mathrm{mg}, 1.42 \mathrm{mmol})$ and $\mathrm{Na}-$ OMe (767 mg, $14.2 \mathrm{mmol}, 28 \%$ solution in $\mathrm{MeOH}$,) in DMF (2.5 mL) was added $\mathrm{CuBr}(20.4 \mathrm{mg}, 0.142 \mathrm{mmol})$. The mixture was stirred at $110{ }^{\circ} \mathrm{C}$ for $2.5 \mathrm{~h}$. The whole was extracted with $\mathrm{CH}_{2} \mathrm{Cl}_{2}$. The extract was washed with brine, and dried over $\mathrm{Na}_{2} \mathrm{SO}_{4}$. After concentration, the residue was purified by flash chromatography over silica gel with $n$-hexane-EtOAc (6:4 to 4:6) to give the titlecompound 23f as colorless solid (171.5 mg, $39.8 \%$ ): $\mathrm{mp} 87.1{ }^{\circ} \mathrm{C}$; IR (neat) $\mathrm{cm}^{-1}$ : 1588 $(\mathrm{C}=\mathrm{N}) ;{ }^{1} \mathrm{H}-\mathrm{NMR}\left(300 \mathrm{MHz}, \mathrm{CDCl}_{3}\right) \delta: 1.38\left(9 \mathrm{H}, \mathrm{s}, 3 \times \mathrm{CH}_{3}\right), 1.87-1.95(2 \mathrm{H}, \mathrm{m}$, $\left.\mathrm{CH}_{2}\right), 3.62\left(2 \mathrm{H}, \mathrm{t}, J=5.6 \mathrm{~Hz}, \mathrm{CH}_{2}\right), 3.86\left(5 \mathrm{H}, \mathrm{m}, \mathrm{CH}_{3}, \mathrm{CH}_{2}\right), 6.92(1 \mathrm{H}, \mathrm{dd}$, $J=8.7,2.7 \mathrm{~Hz}, \mathrm{Ar}), 7.00(1 \mathrm{H}, \mathrm{d}, J=8.4 \mathrm{~Hz}, \mathrm{Ar}), 7.75(1 \mathrm{H}, \mathrm{d}, J=3.3 \mathrm{~Hz}, \mathrm{Ar})$; ${ }^{13} \mathrm{C}-\mathrm{NMR}\left(75 \mathrm{MHz}, \mathrm{CDCl}_{3}\right.$ ) $\delta: 21.9,29.9$ (3C), 45.1, 45.5, 54.1, 55.6, 111.3, 118.9, 120.2, 125.7, 128.9, 138.8, 148.1, 158.1; Anal. calcd for $\mathrm{C}_{16} \mathrm{H}_{21} \mathrm{~N}_{3} \mathrm{OS}$ : C, 63.33; H, 6.98; N, 13.85. Found: C, 63.04; H, 6.97; N, 13.68.

Compound 26f. TFA $(0.88 \mathrm{~mL})$ was added to compound $23 f(26.7 \mathrm{mg}$, $0.088 \mathrm{mmol}$ ). After being stirred under reflux for $3 \mathrm{~h}$, the mixture was added dropwise to $\mathrm{Et}_{3} \mathrm{~N}$ at $0{ }^{\circ} \mathrm{C}$ to adjust $\mathrm{pH}$ to $8-9$. The whole was extracted with EtOAc. The extract was washed with sat. $\mathrm{NaHCO}_{3}$, brine, and dried over $\mathrm{Na}_{2} \mathrm{SO}_{4}$. After concentration, the residue was purified by preparative TLC over aluminum oxide with $n$-hexane-EtOAc (7:3) to give the title compound $\mathbf{2 6 f}$ as colorless solid (9.6 mg, $44 \%): \operatorname{mp~} 89.0{ }^{\circ} \mathrm{C}$; IR (neat) $\mathrm{cm}^{-1}: 1614(\mathrm{C}=\mathrm{N}), 1562(\mathrm{C}=\mathrm{N}) ;{ }^{1} \mathrm{H}-\mathrm{NMR}$ $\left(300 \mathrm{MHz} \mathrm{CDCl}_{3}\right) \delta: 1.93-2.00\left(2 \mathrm{H}, \mathrm{m}, \mathrm{CH}_{2}\right), 3.69\left(2 \mathrm{H}, \mathrm{t}, J=5.4 \mathrm{~Hz}, \mathrm{CH}_{2}\right)$, $3.85\left(3 \mathrm{H}, \mathrm{s}, \mathrm{CH}_{3}\right), 4.02\left(2 \mathrm{H}, \mathrm{t}, J=6.2 \mathrm{~Hz}, \mathrm{CH}_{2}\right), 6.92-6.98(2 \mathrm{H}, \mathrm{m}, \mathrm{Ar}), 7.15(1 \mathrm{H}$, br s, NH), $7.78(1 \mathrm{H}, \mathrm{s}, \mathrm{Ar}) ;{ }^{13} \mathrm{C}-\mathrm{NMR}\left(75 \mathrm{MHz} \mathrm{CDCl}_{3}\right) \delta: 21.0,43.9,44.9,55.6$, $111.9,119.3,119.9,124.8,127.9,146.7,153.9,158.3 ;$ Anal. calcd for $\mathrm{C}_{12} \mathrm{H}_{13} \mathrm{~N}_{3} \mathrm{OS}$ : C, 58.28; H, 5.30; N, 16.99. Found: C, 58.24; H, 5.36; N, 16.46. The purity of the compound was $92 \%$ by HPLC. 


\subsubsection{Synthesis of 3,4-Dihydro-10-methyl-2H, 6H-pyrimido[1,2-c][1,3]benzothiazin-6-imine (26g)}

2-(2-Fluoro-5-methylphenyl)-1,4,5,6-tetrahydropyrimidine (19g). 2-Fluoro-5methylbenzaldehyde $16 \mathrm{~g}(3.0 \mathrm{~g}, 21.7 \mathrm{mmol})$ was subjected to the general procedure as described for $\mathbf{1 8 j}$ to give the title $\mathbf{1 9 g}$ as colorless crystals $(3.1 \mathrm{~g}, 75 \%)$ : mp 119-121 ${ }^{\circ} \mathrm{C}$ (from $\mathrm{CHCl}_{3}-n$-hexane); IR (neat) $\mathrm{cm}^{-1}: 1626(\mathrm{C}=\mathrm{N}) ;{ }^{1} \mathrm{H}-\mathrm{NMR}$ $\left(500 \mathrm{MHz}, \mathrm{CDCl}_{3}\right) \delta: 1.84-1.89\left(2 \mathrm{H}, \mathrm{m}, \mathrm{CH}_{2}\right), 2.31\left(3 \mathrm{H}, \mathrm{s}, \mathrm{CH}_{3}\right), 3.51(4 \mathrm{H}, \mathrm{t}$, $\left.J=5.7 \mathrm{~Hz}, 2 \times \mathrm{CH}_{2}\right), 5.01(1 \mathrm{H}, \mathrm{s}, \mathrm{NH}), 6.92(1 \mathrm{H}, \mathrm{dd}, J=11.7,8.3 \mathrm{~Hz}, \mathrm{Ar})$, 7.09-7.12 (1H, m, Ar), $7.63(1 \mathrm{H}, \mathrm{dd}, J=7.4,2.3 \mathrm{~Hz}, \mathrm{Ar}) ;{ }^{13} \mathrm{C}-\mathrm{NMR}(125 \mathrm{MHz}$, $\left.\mathrm{CDCl}_{3}\right) \delta: 20.5,20.7,42.3(2 \mathrm{C}), 115.6(\mathrm{~d}, J=24.0 \mathrm{~Hz}), 123.7(\mathrm{~d}, J=12.0 \mathrm{~Hz})$, $130.6(\mathrm{~d}, J=3.6 \mathrm{~Hz}), 131.3(\mathrm{~d}, J=9.6 \mathrm{~Hz}), 133.9(\mathrm{~d}, J=3.6 \mathrm{~Hz}), 151.7,158.4$ $(\mathrm{d}, J=244.7 \mathrm{~Hz}) ;{ }^{19} \mathrm{~F}-\mathrm{NMR}\left(500 \mathrm{MHz}, \mathrm{CDCl}_{3}\right) \delta$ : -122.4 ; HRMS (FAB): $m / z$ calcd for $\mathrm{C}_{11} \mathrm{H}_{14} \mathrm{FN}_{2}[\mathrm{M}+\mathrm{H}]^{+}$193.1141; found: 193.1140 .

$\mathrm{N}$-(tert-Butyl)-3,4-dihydro-10-methyl-2H,6H-pyrimido[1,2-c][1,3]benzothiazin-6-imine (23g). To a mixture of compound $19 \mathrm{~g}(0.50 \mathrm{~g}, 2.6 \mathrm{mmol})$ and $\mathrm{KO} t-\mathrm{Bu}(0.58 \mathrm{~g}$, $5.2 \mathrm{mmol})$ in DMAc $(8.7 \mathrm{~mL})$ was added tert-butylisothiocyanate $(0.66 \mathrm{~mL}, 5.2 \mathrm{mmol})$ under an Ar atmosphere. After being stirred at $80^{\circ} \mathrm{C}$ for $3 \mathrm{~h}$, the whole was extracted with EtOAc. The whole was washed with sat. $\mathrm{NaHCO}_{3}$, brine, and dried over $\mathrm{MgSO}_{4}$. After concentration, the residue was purified by flash chromatography over silica gel with $n$-hexaneEtOAc (1:1) to give the title compound $\mathbf{2 3 g}$ as colorless solid $(0.21 \mathrm{~g}, 28 \%)$ : mp $76-77{ }^{\circ} \mathrm{C}$ (from $n$-hexane); IR (neat) $\mathrm{cm}^{-1}: 1597(\mathrm{C}=\mathrm{N}) ;{ }^{1} \mathrm{H}-\mathrm{NMR}\left(400 \mathrm{MHz}, \mathrm{CDCl}_{3}\right) \delta: 1.38(9 \mathrm{H}, \mathrm{s}$, $\left.3 \times \mathrm{CH}_{3}\right), 1.88-1.94\left(2 \mathrm{H}, \mathrm{m}, \mathrm{CH}_{2}\right), 2.33\left(3 \mathrm{H}, \mathrm{s}, \mathrm{CH}_{3}\right), 3.62\left(2 \mathrm{H}, \mathrm{t}, J=5.6 \mathrm{~Hz}, \mathrm{CH}_{2}\right), 3.87$ $\left(2 \mathrm{H}, \mathrm{t}, J=6.2 \mathrm{~Hz}, \mathrm{CH}_{2}\right), 7.00(1 \mathrm{H}, \mathrm{d}, J=8.0 \mathrm{~Hz}, \mathrm{Ar}), 7.13(1 \mathrm{H}, \mathrm{dd}, J=8.0,1.3 \mathrm{~Hz}, \mathrm{Ar})$, $8.01(1 \mathrm{H}, \mathrm{d}, J=1.3 \mathrm{~Hz}, \mathrm{Ar}) ;{ }^{13} \mathrm{C}-\mathrm{NMR}\left(100 \mathrm{MHz}, \mathrm{CDCl}_{3}\right) \delta: 21.0,22.0,29.9$ (3C), 45.1, 45.4, 54.1, 124.4, 125.7, 127.6, 128.6, 131.1, 135.9, 138.7, 148.2; HRMS (FAB): $m / z$ calcd for $\mathrm{C}_{16} \mathrm{H}_{22} \mathrm{~N}_{3} \mathrm{~S}[\mathrm{M}+\mathrm{H}]^{+}$288.1534; found: 288.1535 .

Compound 26g. Using the general procedure as described for 25a, compound 23g (200 mg, $0.7 \mathrm{mmol})$ was allowed to react for $1 \mathrm{~h}$ with TFA $(3.0 \mathrm{~mL})$ and MS4A (450 mg). Purification by preparative TLC over aluminum oxide with $n$ hexane-EtOAc (9:1) gave the title compound $\mathbf{2 6 g}$ as colorless solid (150 $\mathrm{mg}$, $92 \%$ ): $\mathrm{mp} 116{ }^{\circ} \mathrm{C}$ (from $\mathrm{CHCl}_{3}-n$-hexane); IR (neat) $\mathrm{cm}^{-1}: 1623(\mathrm{C}=\mathrm{N}), 1556$ $(\mathrm{C}=\mathrm{N}) ;{ }^{1} \mathrm{H}-\mathrm{NMR}\left(400 \mathrm{MHz}, \mathrm{CDCl}_{3}\right) \delta: 1.94-2.00\left(2 \mathrm{H}, \mathrm{m}, \mathrm{CH}_{2}\right), 2.34(3 \mathrm{H}, \mathrm{s}$, $\left.\mathrm{CH}_{3}\right), 3.69\left(2 \mathrm{H}, \mathrm{t}, J=5.6 \mathrm{~Hz}, \mathrm{CH}_{2}\right), 4.01\left(2 \mathrm{H}, \mathrm{t}, J=6.1 \mathrm{~Hz}, \mathrm{CH}_{2}\right), 6.94(1 \mathrm{H}, \mathrm{d}$, $J=8.0 \mathrm{~Hz}, \mathrm{Ar}), 7.15-7.17(2 \mathrm{H}, \mathrm{m}, \mathrm{Ar}, \mathrm{NH}), 8.04(1 \mathrm{H}, \mathrm{s}, \mathrm{Ar}) ;{ }^{13} \mathrm{C}-\mathrm{NMR}$ $\left(100 \mathrm{MHz}, \mathrm{CDCl}_{3}\right) \delta: 21.0,21.1,43.8,44.9,123.5,125.4,126.5,129.0,131.6$, 136.3, 146.9, 153.7; HRMS (FAB): $m / z$ calcd for $\mathrm{C}_{12} \mathrm{H}_{14} \mathrm{~N}_{3} \mathrm{~S}[\mathrm{M}+\mathrm{H}]^{+} 232.0908$; found: 232.0913. 


\subsubsection{Synthesis of 10-Bromo-3,4-dihydro-2H, 6H-pyrimido[1,2-c][1,3]benzothiazin-6-imine (26k)}

2-(5-Bromo-2-fluorophenyl)-1,4,5,6-tetrahydropyrimidine (19k). 5-Bromo-2fluorobenzaldehyde $16 \mathrm{k}(1.02 \mathrm{~g}, 5.0 \mathrm{mmol})$ was subjected to the general procedure as described for $\mathbf{1 8} \mathbf{j}$ to give the title compound $\mathbf{1 9 k}$ as colorless crystals (1.02 g, $79 \%$ ): mp 121-122 ${ }^{\circ} \mathrm{C}$ (from $\mathrm{CHCl}_{3}-n$-hexane); IR (neat) $\mathrm{cm}^{-1}: 1623$ $(\mathrm{C}=\mathrm{N}) ;{ }^{1} \mathrm{H}-\mathrm{NMR}\left(400 \mathrm{MHz}, \mathrm{CDCl}_{3}\right) \delta: 1.83-1.89\left(2 \mathrm{H}, \mathrm{m}, \mathrm{CH}_{2}\right), 3.50(4 \mathrm{H}, \mathrm{t}$, $\left.J=5.7 \mathrm{~Hz}, 2 \times \mathrm{CH}_{2}\right), 5.28(1 \mathrm{H}$, br s, NH), $6.94(1 \mathrm{H}, \mathrm{dd}, J=11.1,8.8 \mathrm{~Hz}, \mathrm{Ar})$, $7.42(1 \mathrm{H}$, ddd, $J=8.8,4.4,2.7 \mathrm{~Hz}, \mathrm{Ar}), 7.97(1 \mathrm{H}, \mathrm{dd}, J=6.8,2.7 \mathrm{~Hz}, \mathrm{Ar}) ;{ }^{13} \mathrm{C}-$ NMR (100 MHz, $\left.\mathrm{CDCl}_{3}\right) \delta: 20.6,42.1(2 \mathrm{C}), 117.1(\mathrm{~d}, J=3.3 \mathrm{~Hz}), 117.7$ (d, $J=25.7 \mathrm{~Hz}), 126.2(\mathrm{~d}, \quad J=13.2 \mathrm{~Hz}), 133.3 \quad(\mathrm{~d}, \quad J=3.3 \mathrm{~Hz}), 133.6 \quad(\mathrm{~d}$, $J=9.1 \mathrm{~Hz}), 150.3,159.1(\mathrm{~d}, J=247.5 \mathrm{~Hz}) ;{ }^{19} \mathrm{~F}-\mathrm{NMR}\left(500 \mathrm{MHz}, \mathrm{CDCl}_{3}\right) \delta$ : -119.5; Anal. calcd for $\mathrm{C}_{10} \mathrm{H}_{10} \mathrm{BrFN}_{2}$ : C, 46.72; H, 3.92; N, 10.90. Found: C, 46.59; H, 3.87; N, 10.89 .

10-Bromo- $N$-(tert-butyl)-3,4-dihydro-2H,6H-pyrimido[1,2-c][1,3]benzothiazin-6-imine (23k). Using the general procedure as described for $22 \mathrm{e}$, compound $19 \mathrm{k}(257.1 \mathrm{mg}, 1.00 \mathrm{mmol})$ was allowed to react at $\mathrm{rt}$ overnight. Purification by flash chromatography over aluminum oxide with $n$-hexane-EtOAc (1:0 to 9:1) gave the title compound 23k as colorless solid (111.6 mg, $32 \%): \mathrm{mp} 93-94{ }^{\circ} \mathrm{C}$ (from $n$-hexane); IR (neat) $\mathrm{cm}^{-1}: 1599(\mathrm{C}=\mathrm{N}) ;{ }^{1} \mathrm{H}-\mathrm{NMR}\left(400 \mathrm{MHz}, \mathrm{CDCl}_{3}\right) \delta$ : $1.38\left(9 \mathrm{H}, \mathrm{s}, 3 \times \mathrm{CH}_{3}\right), 1.88-1.93\left(2 \mathrm{H}, \mathrm{m}, \mathrm{CH}_{2}\right), 3.62\left(2 \mathrm{H}, \mathrm{t}, J=5.6 \mathrm{~Hz}, \mathrm{CH}_{2}\right)$, $3.86\left(2 \mathrm{H}, \mathrm{t}, J=6.1 \mathrm{~Hz}, \mathrm{CH}_{2}\right), 6.97(1 \mathrm{H}, \mathrm{d}, J=8.5 \mathrm{~Hz}, \mathrm{Ar}), 7.41(1 \mathrm{H}, \mathrm{dd}$, $J=8.5,2.2 \mathrm{~Hz}, \mathrm{Ar}), 8.36(1 \mathrm{H}, \mathrm{d}, J=2.2 \mathrm{~Hz}, \mathrm{Ar}) ;{ }^{13} \mathrm{C}-\mathrm{NMR}\left(100 \mathrm{MHz}, \mathrm{CDCl}_{3}\right)$ $\delta: 21.8,29.9$ (3C), 45.1, 45.4, 54.2, 119.7, 125.9, 128.1, 129.3, 131.2, 133.0, 137.4, 146.7; Anal. calcd for $\mathrm{C}_{15} \mathrm{H}_{18} \mathrm{BrN}_{3} \mathrm{~S}$ : C, 51.14; H, 5.15; N, 11.93. Found: C, 51.09; H, 4.98; N, 11.89 .

Compound 26k. Using the general procedure as described for $25 \mathrm{a}$, compound $23 \mathrm{k}(52.8 \mathrm{mg}, 0.15 \mathrm{mmol})$ was allowed to react for $2 \mathrm{~h}$ with TFA $(1.5 \mathrm{~mL})$ and MS4A (225 mg). Purification by flash chromatography over silica gel with $n$-hexane-EtOAc $(2: 1)$ gave the title compound $\mathbf{2 6 k}$ as colorless crystals $(39.7 \mathrm{mg}$, $89 \%$ ): $\mathrm{mp} 106-107{ }^{\circ} \mathrm{C}$ (from $\mathrm{CHCl}_{3}-n$-hexane); IR (neat) $\mathrm{cm}^{-1}$ : $1621(\mathrm{C}=\mathrm{N})$, $1571(\mathrm{C}=\mathrm{N}) ;{ }^{1} \mathrm{H}-\mathrm{NMR}\left(500 \mathrm{MHz}, \mathrm{CDCl}_{3}\right) \delta: 1.94-1.99\left(2 \mathrm{H}, \mathrm{m}, \mathrm{CH}_{2}\right), 3.69(2 \mathrm{H}, \mathrm{t}$, $\left.J=5.4 \mathrm{~Hz}, \mathrm{CH}_{2}\right), 4.01\left(2 \mathrm{H}, \mathrm{t}, J=6.3 \mathrm{~Hz}, \mathrm{CH}_{2}\right), 6.91(1 \mathrm{H}, \mathrm{d}, J=8.6 \mathrm{~Hz}, \mathrm{Ar})$, $7.20(1 \mathrm{H}$, br s, NH), $7.44(1 \mathrm{H}, \mathrm{dd}, J=8.6,2.3 \mathrm{~Hz}, \mathrm{Ar}), 8.39(1 \mathrm{H}, \mathrm{d}, J=2.3 \mathrm{~Hz}$, $\mathrm{Ar}) ;{ }^{13} \mathrm{C}-\mathrm{NMR}\left(125 \mathrm{MHz}, \mathrm{CDCl}_{3}\right) \delta: 20.9,43.8,44.9,120.0,125.0,127.8,128.3$, 131.6, 133.5, 145.4, 152.5; Anal. calcd for $\mathrm{C}_{11} \mathrm{H}_{10} \mathrm{BrN}_{3} \mathrm{~S}: \mathrm{C}, 44.61 ; \mathrm{H}, 3.40 ; \mathrm{N}$, 14.19. Found: C, 44.51; H, 3.66; N, 14.06 . 


\subsubsection{Synthesis of 3,4-Dihydro-10-phenyl-2H, 6H-pyrimido[1,2-c][1,3]benzothiazin-6-imine (26l)}

$N$-(tert-Butyl)-3,4-dihydro-10-phenyl-2H,6H-pyrimido[1,2-c][1,3]benzothiazin-6-imine (23l). Using the general procedure as described for 221, 10-bromo- $\mathrm{N}$ (tert-butyl)-3,4-dihydro-2H,6H-pyrimido[1,2-c][1,3]benzothiazin-6-imine $\quad \mathbf{2 3 k}$ $(52.8 \mathrm{mg}, 0.15 \mathrm{mmol})$ was allowed to react for $1 \mathrm{~h}$. Purification by flash chromatography over aluminum oxide with $n$-hexane-EtOAc (1:0 to 9:1) gave the title compound $23 \mathrm{I}$ as colorless solid (32.6 mg, $62 \%$ ): $\mathrm{mp} 101-103{ }^{\circ} \mathrm{C}$ (from $n$-hexane); IR (neat) $\mathrm{cm}^{-1}: 1594(\mathrm{C}=\mathrm{N}) ;{ }^{1} \mathrm{H}-\mathrm{NMR}\left(500 \mathrm{MHz}, \mathrm{CDCl}_{3}\right) \delta: 1.40(9 \mathrm{H}, \mathrm{s}$, $\left.3 \times \mathrm{CH}_{3}\right), 1.90-1.95\left(2 \mathrm{H}, \mathrm{m}, \mathrm{CH}_{2}\right), 3.64\left(2 \mathrm{H}, \mathrm{t}, J=5.4 \mathrm{~Hz}, \mathrm{CH}_{2}\right), 3.89(2 \mathrm{H}, \mathrm{t}$, $\left.J=6.0 \mathrm{~Hz}, \mathrm{CH}_{2}\right), 7.18(1 \mathrm{H}, \mathrm{d}, J=8.0 \mathrm{~Hz}, \mathrm{Ar}), 7.32(1 \mathrm{H}, \mathrm{t}, J=7.4 \mathrm{~Hz}, \mathrm{Ar}), 7.41$ $(2 \mathrm{H}, \mathrm{t}, J=7.4 \mathrm{~Hz}, \mathrm{Ar}), 7.55(1 \mathrm{H}, \mathrm{dd}, J=8.0,2.0 \mathrm{~Hz}, \mathrm{Ar}), 7.61(2 \mathrm{H}, \mathrm{d}$, $J=7.4 \mathrm{~Hz}, \mathrm{Ar}), 8.47(1 \mathrm{H}, \mathrm{d}, J=2.0 \mathrm{~Hz}, \mathrm{Ar}) ;{ }^{13} \mathrm{C}-\mathrm{NMR}\left(125 \mathrm{MHz}, \mathrm{CDCl}_{3}\right) \delta$ : 21.9, 30.0 (3C), 45.1, 45.5, 54.2, 125.0, 126.9, 127.0 (2C), 127.4, 127.9, 128.1, 128.7 (2C), 128.7, 138.2, 139.1, 140.0, 147.9; HRMS (FAB): $\mathrm{m} / \mathrm{z}$ calcd for $\mathrm{C}_{21} \mathrm{H}_{24} \mathrm{~N}_{3} \mathrm{~S}[\mathrm{M}+\mathrm{H}]^{+} 350.1691$; found:350.1683.

Compound 26l. Using the general procedure as described for 25a, compound $23 \mathrm{l}(13.1 \mathrm{mg}, 0.037 \mathrm{mmol})$ was allowed to react for $2 \mathrm{~h}$ with TFA (1.0 mL) and MS4A (150 mg). Purification by flash chromatography over aluminum oxide with n-hexane-EtOAc (9:1) gave the title compound 261 as colorless solid (8.4 $\mathrm{mg}$, $77 \%$ ): $\mathrm{mp} 82{ }^{\circ} \mathrm{C}$ (from $\mathrm{CHCl}_{3}-n$-hexane); IR (neat) $\mathrm{cm}^{-1}: 1621(\mathrm{C}=\mathrm{N}), 1550$ $(\mathrm{C}=\mathrm{N}) ;{ }^{1} \mathrm{H}-\mathrm{NMR}\left(400 \mathrm{MHz}, \mathrm{CDCl}_{3}\right) \delta: 1.95-2.01\left(2 \mathrm{H}, \mathrm{m}, \mathrm{CH}_{2}\right), 3.71(2 \mathrm{H}, \mathrm{t}$, $\left.J=5.6 \mathrm{~Hz}, \mathrm{CH}_{2}\right), 4.03\left(2 \mathrm{H}, \mathrm{t}, J=6.2 \mathrm{~Hz}, \mathrm{CH}_{2}\right), 7.10-7.44(5 \mathrm{H}, \mathrm{m}, \mathrm{Ar})$, 7.56-7.64 (3H, m, Ar), $8.50(1 \mathrm{H}, \mathrm{d}, J=2.2 \mathrm{~Hz}, \mathrm{Ar}) ;{ }^{13} \mathrm{C}-\mathrm{NMR}(100 \mathrm{MHz}$, $\left.\mathrm{CDCl}_{3}\right) \delta: 21.1,43.9,45.0,124.0,126.9,127.1$ (2C), 127.3, 127.6, 128.8 (2C), 129.2, 139.4, 139.8, 146.6, 152.1, 153.3; Anal. calcd for $\mathrm{C}_{17} \mathrm{H}_{15} \mathrm{~N}_{3} \mathrm{~S}: \mathrm{C}, 69.59 ; \mathrm{H}$, 5.15; N, 14.32. Found: C, 69.61; H, 5.13; N, 14.22.

\subsubsection{Synthesis of 3,4-Dihydro-10-vinyl-2H, 6H-pyrimido[1,2-c][1,3]benzothiazin-6-imine (26m)}

$N$-(tert-Butyl)-3,4-dihydro-10-vinyl-2H,6H-pyrimido[1,2-c][1,3]benzothiazin6-imine $\mathbf{( 2 3 m )}$. Using the general procedure as described for 221, 10-bromo- $N$ (tert-butyl)-3,4-dihydro-2H,6H-pyrimido[1,2-c][1,3]benzothiazin-6-imine $\quad \mathbf{2 3 k}$ (52.8 $\mathrm{mg}, 0.15 \mathrm{mmol}$ ) was allowed to react with vinylboronic acid pinacol ester $(0.031 \mathrm{~mL}, 0.18 \mathrm{mmol})$ for $1 \mathrm{~h}$. Purification by flash chromatography over aluminum oxide with $n$-hexane-EtOAc (1:0 to 9:1) gave the title compound $\mathbf{2 3 m}$ as a colorless oil $(30.5 \mathrm{mg}, 68 \%)$ : IR (neat) $\mathrm{cm}^{-1}: 1595(\mathrm{C}=\mathrm{N}) ;{ }^{1} \mathrm{H}-\mathrm{NMR}(400 \mathrm{MHz}$, $\left.\mathrm{CDCl}_{3}\right) \delta: 1.38\left(9 \mathrm{H}, \mathrm{s}, 3 \times \mathrm{CH}_{3}\right), 1.89-1.94\left(2 \mathrm{H}, \mathrm{m}, \mathrm{CH}_{2}\right), 3.63(2 \mathrm{H}, \mathrm{t}$, $\left.J=5.4 \mathrm{~Hz}, \mathrm{CH}_{2}\right), 3.87\left(2 \mathrm{H}, \mathrm{t}, J=6.0 \mathrm{~Hz}, \mathrm{CH}_{2}\right), 5.24(1 \mathrm{H}, \mathrm{d}, J=11.0 \mathrm{~Hz}, \mathrm{CH})$, $5.77(1 \mathrm{H}, \mathrm{d}, J=17.6 \mathrm{~Hz}, \mathrm{CH}), 6.69(1 \mathrm{H}, \mathrm{dd}, J=17.6,11.0 \mathrm{~Hz}, \mathrm{CH}), 7.07(1 \mathrm{H}, \mathrm{d}$, 
$J=8.3 \mathrm{~Hz}, \mathrm{Ar}), 7.40(1 \mathrm{H}, \mathrm{dd}, J=8.3,2.0 \mathrm{~Hz}, \mathrm{Ar}), 8.20(1 \mathrm{H}, \mathrm{d}, J=2.0 \mathrm{~Hz}, \mathrm{Ar})$;

${ }^{13} \mathrm{C}-\mathrm{NMR}\left(100 \mathrm{MHz}, \mathrm{CDCl}_{3}\right.$ ) $\delta: 21.9,30.0$ (3C), 45.1, 45.4, 54.1, 114.1, 124.7, 126.6, 127.4, 127.8, 128.2, 135.7, 135.9, 138.2, 147.9; HRMS (FAB): $m / z$ calcd for $\mathrm{C}_{17} \mathrm{H}_{22} \mathrm{~N}_{3} \mathrm{~S}[\mathrm{M}+\mathrm{H}]^{+}$300.1534; found: 300.1532 .

Compound $\mathbf{2 6} \mathbf{m}$. Using the general procedure as described for $\mathbf{2 5 a}$, compound 23m $(7.3 \mathrm{mg}, 0.024 \mathrm{mmol})$ was allowed to react for $1 \mathrm{~h}$ with TFA $(1.0 \mathrm{~mL})$ and MS4A (150 mg). Purification by flash chromatography over aluminum oxide with $n$-hexane-EtOAc (9:1) gave the title compound $\mathbf{2 6 m}$ as colorless solid (3.7 $\mathbf{m g}$, $62 \%$ ): $\mathrm{mp} 69-70{ }^{\circ} \mathrm{C}$ (from $\mathrm{CHCl}_{3}-n$-hexane); IR (neat) $\mathrm{cm}^{-1}: 1622(\mathrm{C}=\mathrm{N}), 1550$ $(\mathrm{C}=\mathrm{N}) ;{ }^{1} \mathrm{H}-\mathrm{NMR}\left(400 \mathrm{MHz}, \mathrm{CDCl}_{3}\right) \delta: 1.95-2.01\left(2 \mathrm{H}, \mathrm{m}, \mathrm{CH}_{2}\right), 3.70(2 \mathrm{H}, \mathrm{t}$, $\left.J=5.7 \mathrm{~Hz}, \mathrm{CH}_{2}\right), 4.02\left(2 \mathrm{H}, \mathrm{t}, J=6.2 \mathrm{~Hz}, \mathrm{CH}_{2}\right), 5.27(1 \mathrm{H}, \mathrm{dd}, J=10.7,0.6 \mathrm{~Hz}$, $\mathrm{CH}), 5.79(1 \mathrm{H}, \mathrm{dd}, J=17.7,0.6 \mathrm{~Hz}, \mathrm{CH}), 6.69(1 \mathrm{H}, \mathrm{dd}, J=17.7,10.7 \mathrm{~Hz}, \mathrm{CH})$, $7.00(1 \mathrm{H}, \mathrm{d}, J=8.3 \mathrm{~Hz}, \mathrm{Ar}), 7.19(1 \mathrm{H}$, br s, NH$), 7.42(1 \mathrm{H}, \mathrm{dd}, J=8.3,2.0 \mathrm{~Hz}$, Ar), $8.23(1 \mathrm{H}, \mathrm{d}, J=2.0 \mathrm{~Hz}, \mathrm{Ar}) ;{ }^{13} \mathrm{C}-\mathrm{NMR}\left(100 \mathrm{MHz}, \mathrm{CDCl}_{3}\right) \delta: 21.0,43.8$, $44.9,114.5,123.8,126.8,126.9,127.8,127.9,135.6,135.9,146.5,153.2$; HRMS (FAB): $m / z$ calcd for $\mathrm{C}_{13} \mathrm{H}_{14} \mathrm{~N}_{3} \mathrm{~S}[\mathrm{M}+\mathrm{H}]^{+}$244.0908; found: 244.0902 .

\subsubsection{Synthesis of 10-(4-Benzoylphenyl)-3,4-dihydro-2H, 6H-pyrimido[1,2-c][1,3]benzothiazin-6-imine (26q)}

10-(4-Benzoylphenyl)- $N$-(tert-butyl)-3,4-dihydro-2H,6H-pyrimido[1,2-c][1,3]benzothiazin-6-imine (23q). Using the general procedure as described for 221, 10-bromo- $N$-(tert-butyl)-3,4-dihydro-2H,6H-pyrimido[1,2-c][1,3]benzothiazin-6imine 23k (52.8 mg, $0.15 \mathrm{mmol}$ ) was allowed to react with 4-benzoylphenylboronic acid (40.7 mg, $0.18 \mathrm{mmol}$ ) for $1 \mathrm{~h}$. Purification by flash chromatography over aluminum oxide with $n$-hexane-EtOAc (8:2) gave the title compound $\mathbf{2 3 q}$ as colorless solid (65.1 mg, $96 \%$ ): mp $192-193{ }^{\circ} \mathrm{C}$ (from $\mathrm{CHCl}_{3}-n$-hexane); IR (neat) $\mathrm{cm}^{-1}$ : $1654(\mathrm{C}=\mathrm{O}), 1592(\mathrm{C}=\mathrm{N}) ;{ }^{1} \mathrm{H}-\mathrm{NMR}\left(400 \mathrm{MHz}, \mathrm{CDCl}_{3}\right) \delta: 1.41\left(9 \mathrm{H}, \mathrm{s}, 3 \times \mathrm{CH}_{3}\right)$, 1.91-1.96 (2H, m, $\left.\mathrm{CH}_{2}\right), 3.65\left(2 \mathrm{H}, \mathrm{t}, J=5.5 \mathrm{~Hz}, \mathrm{CH}_{2}\right), 3.90(2 \mathrm{H}, \mathrm{t}, J=6.1 \mathrm{~Hz}$, $\left.\mathrm{CH}_{2}\right), 7.39(1 \mathrm{H}, \mathrm{d}, J=1.7 \mathrm{~Hz}, \mathrm{Ar}), 7.46-7.52(3 \mathrm{H}, \mathrm{m}, \mathrm{Ar}), 7.58-7.62(1 \mathrm{H}, \mathrm{m}, \mathrm{Ar})$, $7.70(2 \mathrm{H}, \mathrm{d}, J=8.5 \mathrm{~Hz}, \mathrm{Ar}), 7.82(2 \mathrm{H}, \mathrm{dd}, J=8.3,1.2 \mathrm{~Hz}, \operatorname{Ar}), 7.88(2 \mathrm{H}, \mathrm{d}$, $J=8.5 \mathrm{~Hz}, \mathrm{Ar}), 8.30(1 \mathrm{H}, \mathrm{d}, J=8.5 \mathrm{~Hz}, \mathrm{Ar}) ;{ }^{13} \mathrm{C}-\mathrm{NMR}\left(125 \mathrm{MHz}, \mathrm{CDCl}_{3}\right) \delta$ : 21.9, 30.0 (3C), 45.1, 45.4, 54.2, 123.0, 124.8, 126.9 (2C), 127.3, 128.3 (2C), 129.1, 129.8, 130.0 (2C), 130.7 (2C), 132.5, 136.9, 137.6, 137.9, 141.6, 143.3, 147.5, 196.1; HRMS (FAB): $m / z$ calcd for $\mathrm{C}_{28} \mathrm{H}_{28} \mathrm{~N}_{3} \mathrm{OS}[\mathrm{M}+\mathrm{H}]^{+} 454.1953$; found: 454.1952.

Compound 26q. Using the general procedure as described for 25a, compound 23q $(36.2 \mathrm{mg}, 0.08 \mathrm{mmol})$ was allowed to react for $1 \mathrm{~h}$ with TFA $(1.0 \mathrm{~mL})$ and MS4A (150 mg). Purification by flash chromatography over aluminum oxide with n-hexane-EtOAc (7:3) gave the title compound 26q as colorless solid (23.4 $\mathrm{mg}$, $74 \%$ ): $\mathrm{mp} 163-165^{\circ} \mathrm{C}$ (from $\mathrm{CHCl}_{3}-n$-hexane); IR (neat) $\mathrm{cm}^{-1}: 1654(\mathrm{C}=\mathrm{O})$, 
$1622(\mathrm{C}=\mathrm{N}), 1561(\mathrm{C}=\mathrm{N}) ;{ }^{1} \mathrm{H}-\mathrm{NMR}\left(400 \mathrm{MHz}, \mathrm{CDCl}_{3}\right) \delta: 1.97-2.03(2 \mathrm{H}, \mathrm{m}$, $\left.\mathrm{CH}_{2}\right), 3.72\left(2 \mathrm{H}, \mathrm{t}, J=5.6 \mathrm{~Hz}, \mathrm{CH}_{2}\right), 4.05\left(2 \mathrm{H}, \mathrm{t}, J=6.2 \mathrm{~Hz}, \mathrm{CH}_{2}\right), 7.15(1 \mathrm{H}, \mathrm{d}$, $J=8.0 \mathrm{~Hz}, \mathrm{Ar}), 7.48-7.52(2 \mathrm{H}, \mathrm{m}, \mathrm{Ar}), 7.58-7.64(2 \mathrm{H}, \mathrm{m}, \mathrm{Ar}), 7.73(2 \mathrm{H}, \mathrm{d}$, $J=8.5 \mathrm{~Hz}, \mathrm{Ar}), 7.82(2 \mathrm{H}, \mathrm{dd}, J=8.2,1.3 \mathrm{~Hz}, \mathrm{Ar}), 7.88(2 \mathrm{H}, \mathrm{d}, J=8.5 \mathrm{~Hz}, \mathrm{Ar})$, $8.57(1 \mathrm{H}, \mathrm{d}, J=2.0 \mathrm{~Hz}, \mathrm{Ar}) ;{ }^{13} \mathrm{C}-\mathrm{NMR}\left(100 \mathrm{MHz}, \mathrm{CDCl}_{3}\right) \delta: 21.0,43.9,45.0$, $124.3,126.7$ (2C), 127.2, 127.6, 128.3 (2C), 128.8, 129.2, 130.0 (2C), 130.7 (2C), 132.4, 136.5, 137.7, 138.0, 143.7, 146.3, 152.9, 196.1; HRMS (FAB): $m / z$ calcd for $\mathrm{C}_{24} \mathrm{H}_{20} \mathrm{~N}_{3} \mathrm{OS}[\mathrm{M}+\mathrm{H}]^{+}$398.1327; found: 398.1327 .

\subsubsection{Synthesis of 11-Fluoro-3,4-dihydro-2H, 6H-pyrimido[1,2-c][1,3]benzothiazin-6-imine (27i)}

2-(2,6-Difluorophenyl)-1,4,5,6-tetrahydropyrimidine (20i). 2,6-Difluorobenzaldehyde $17 \mathbf{i}(1.00 \mathrm{~g}, 7.04 \mathrm{mmol})$ was subjected to the general procedure as described for $\mathbf{1 8 j}$ to give the title compound $\mathbf{2 0} \mathbf{i}$ as colorless crystals $(1.08 \mathrm{~g}$, $78 \%$ ): mp $165-166{ }^{\circ} \mathrm{C}$ (from $\mathrm{CHCl}_{3}-n$-hexane); IR (neat) $\mathrm{cm}^{-1}: 1620(\mathrm{C}=\mathrm{N}) ;{ }^{1} \mathrm{H}$ NMR $\left(500 \mathrm{MHz}, \mathrm{CDCl}_{3}\right) \delta: 1.85-1.90\left(2 \mathrm{H}, \mathrm{m}, \mathrm{CH}_{2}\right), 3.47(4 \mathrm{H}, \mathrm{t}, J=5.7 \mathrm{~Hz}$, $\left.2 \times \mathrm{CH}_{2}\right), 4.77(1 \mathrm{H}$, br s, NH), 6.86-6.91 (2H, m, Ar), 7.24-7.30 (1H, m, Ar); ${ }^{13} \mathrm{C}-\mathrm{NMR}\left(100 \mathrm{MHz}, \mathrm{CDCl}_{3}\right) \delta: 20.5,42.2(2 \mathrm{C}), 111.4-111.6(\mathrm{~m}, 2 \mathrm{C}), 115.9(\mathrm{t}$, $J=20.3 \mathrm{~Hz}), 130.1(\mathrm{t}, J=9.9 \mathrm{~Hz}), 146.8,160.3(\mathrm{dd}, J=250.3,7.0 \mathrm{~Hz}, 2 \mathrm{C})$. ${ }^{19} \mathrm{~F}-\mathrm{NMR}\left(500 \mathrm{MHz}, \mathrm{CDCl}_{3}\right) \delta:-114.4 ;$ Anal. calcd for $\mathrm{C}_{10} \mathrm{H}_{10} \mathrm{~F}_{2} \mathrm{~N}_{2}: \mathrm{C}, 61.22 ; \mathrm{H}$, 5.14; N, 14.28. Found: C, 61.23; H, 5.13; N, 14.26.

$N$-(tert-Butyl)-11-fluoro-3,4-dihydro-2H,6H-pyrimido[1,2-c][1,3]benzothiazin-6-imine (24i). Using the general procedure as described for 22e, compound 20i (196.2 $\mathrm{mg}, 1.0 \mathrm{mmol}$ ) was allowed to react at $\mathrm{rt}$ overnight. Purification by flash chromatography over aluminum oxide with $n$-hexane-EtOAc (1:0 to 9:1) gave the title compound $\mathbf{2 4 i}$ as colorless solid $\left(212.6 \mathrm{mg}, 73 \%\right.$ ): $\mathrm{mp} 81{ }^{\circ} \mathrm{C}$ (from $n$-hexane); IR (neat) $\mathrm{cm}^{-1}: 1592(\mathrm{C}=\mathrm{N}) ;{ }^{1} \mathrm{H}-\mathrm{NMR}\left(500 \mathrm{MHz}, \mathrm{CDCl}_{3}\right) \delta: 1.37(9 \mathrm{H}, \mathrm{s}$, $\left.3 \times \mathrm{CH}_{3}\right), 1.90-1.95\left(2 \mathrm{H}, \mathrm{m}, \mathrm{CH}_{2}\right), 3.66\left(2 \mathrm{H}, \mathrm{t}, J=5.7 \mathrm{~Hz}, \mathrm{CH}_{2}\right), 3.80(2 \mathrm{H}, \mathrm{t}$, $\left.J=6.6 \mathrm{~Hz}, \mathrm{CH}_{2}\right), 6.94-6.99(2 \mathrm{H}, \mathrm{m}, \mathrm{Ar}), 7.23-7.26(1 \mathrm{H}, \mathrm{m}, \mathrm{Ar}) ;{ }^{13} \mathrm{C}-\mathrm{NMR}$ $\left(125 \mathrm{MHz}, \mathrm{CDCl}_{3}\right) \delta: 22.5,30.1(3 \mathrm{C}), 45.3,45.5,54.2,115.0(\mathrm{~d}, J=24.0 \mathrm{~Hz})$, $118.7,120.9(\mathrm{~d}, J=3.6 \mathrm{~Hz}), 130.6(\mathrm{~d}, J=10.8 \mathrm{~Hz}), 131.9,137.6,146.1(\mathrm{~d}$, $J=8.4 \mathrm{~Hz}), 160.2(\mathrm{~d}, J=260.3 \mathrm{~Hz}) ;{ }^{19} \mathrm{~F}-\mathrm{NMR}\left(500 \mathrm{MHz}, \mathrm{CDCl}_{3}\right) \delta:-110.8$; HRMS (FAB): $m / z$ calcd for $\mathrm{C}_{15} \mathrm{H}_{19} \mathrm{FN}_{3} \mathrm{~S}[\mathrm{M}+\mathrm{H}]^{+} 292.1284$; found: 292.1288 .

Compound 27i. Using the general procedure as described for $25 \mathrm{a}$, compound $24 \mathbf{i}(58.3 \mathrm{mg}, 0.20 \mathrm{mmol})$ was allowed to react for $1 \mathrm{~h}$. Purification by flash chromatography over aluminum oxide with $n$-hexane-EtOAc $(9: 1)$ gave the title compound 27i as colorless solid (42.3 mg, $90 \%$ ): $\mathrm{mp} 142.5^{\circ} \mathrm{C}$ (from $\mathrm{CHCl}_{3}-n$ hexane); IR (neat) $\mathrm{cm}^{-1}: 1624(\mathrm{C}=\mathrm{N}), 1585(\mathrm{C}=\mathrm{N}) ;{ }^{1} \mathrm{H}-\mathrm{NMR}\left(500 \mathrm{MHz}, \mathrm{CDCl}_{3}\right)$ $\delta$ : 1.97-2.02 (2H, m, $\left.\mathrm{CH}_{2}\right), 3.73\left(2 \mathrm{H}, \mathrm{t}, J=5.2 \mathrm{~Hz}, \mathrm{CH}_{2}\right), 3.94(2 \mathrm{H}, \mathrm{t}$, $\left.J=6.6 \mathrm{~Hz}, \mathrm{CH}_{2}\right), 6.91(1 \mathrm{H}, \mathrm{d}, J=8.0 \mathrm{~Hz}, \mathrm{Ar}), 6.97-7.01(1 \mathrm{H}, \mathrm{m}, \mathrm{Ar}), 7.22(1 \mathrm{H}$, br s, NH), 7.27-7.31 (1H, m, Ar); ${ }^{13} \mathrm{C}-\mathrm{NMR}\left(125 \mathrm{MHz}, \mathrm{CDCl}_{3}\right) \delta: 21.7,44.0$, 
45.5, $115.3(\mathrm{~d}, J=24.0 \mathrm{~Hz}), 117.4(\mathrm{~d}, J=8.4 \mathrm{~Hz}), 120.0(\mathrm{~d}, J=3.6 \mathrm{~Hz}), 131.2$ $(\mathrm{d}, J=9.6 \mathrm{~Hz}), 131.5,144.8(\mathrm{~d}, J=9.6 \mathrm{~Hz}), 152.6(\mathrm{~d}, J=4.8 \mathrm{~Hz}), 160.5(\mathrm{~d}$, $J=261.5 \mathrm{~Hz}) ;{ }^{19} \mathrm{~F}-\mathrm{NMR}\left(500 \mathrm{MHz}, \mathrm{CDCl}_{3}\right) \delta:-110.0$. Anal. calcd for $\mathrm{C}_{11} \mathrm{H}_{10} \mathrm{FN}_{3} \mathrm{~S}$ : C, 56.15; H, 4.28; N, 17.86. Found: C, 56.05; H, 4.28; N, 17.71.

\subsubsection{Synthesis of 8-Bromo-3,4-dihydro-2H, $6 H$-pyrimido[1,2-c][1,3]benzothiazin-6-imine (27k)}

2-(3-Bromo-2-fluorophenyl)-1,4,5,6-tetrahydropyrimidine (20k). 3-Bromo-2fluorobenzaldehyde $17 \mathrm{k}(0.71 \mathrm{~g}, 3.5 \mathrm{mmol})$ was subjected to the general procedure as described for $\mathbf{1 8} \mathbf{j}$ to give the title compound $\mathbf{2 0 k}$ as colorless crystals (0.62 g, $69 \%)$ : mp $99{ }^{\circ} \mathrm{C}$; IR (neat) $\mathrm{cm}^{-1}: 1624(\mathrm{C}=\mathrm{N}) ;{ }^{1} \mathrm{H}-\mathrm{NMR}(400 \mathrm{MHz}$, $\left.\mathrm{CDCl}_{3}\right) \delta: 1.84-1.89\left(2 \mathrm{H}, \mathrm{m}, \mathrm{CH}_{2}\right), 3.50\left(4 \mathrm{H}, \mathrm{t}, J=5.7 \mathrm{~Hz}, 2 \times \mathrm{CH}_{2}\right), 5.13(1 \mathrm{H}$, br s, NH), $7.03(1 \mathrm{H}, \mathrm{td}, J=8.0,0.9 \mathrm{~Hz}, \mathrm{Ar}), 7.54(1 \mathrm{H}, \mathrm{ddd}, J=8.0,6.4,1.3 \mathrm{~Hz}$, Ar), $7.69(1 \mathrm{H}, \mathrm{ddd}, J=8.0,6.5,1.3 \mathrm{~Hz}, \mathrm{Ar}) ;{ }^{13} \mathrm{C}-\mathrm{NMR}\left(100 \mathrm{MHz}, \mathrm{CDCl}_{3}\right) \delta$ : 20.6, $42.1(2 \mathrm{C}), 109.6(\mathrm{~d}, J=22.3 \mathrm{~Hz}), 125.1(\mathrm{~d}, J=4.1 \mathrm{~Hz}), 126.3(\mathrm{~d}$, $J=13.2 \mathrm{~Hz}), 129.8(\mathrm{~d}, J=3.3 \mathrm{~Hz}), 134.3,150.8,156.3(\mathrm{~d}, J=248.3 \mathrm{~Hz}) ;{ }^{19} \mathrm{~F}-$ NMR $\left(500 \mathrm{MHz}, \mathrm{CDCl}_{3}\right) \delta:-110.7$; Anal. calcd for $\mathrm{C}_{10} \mathrm{H}_{10} \mathrm{BrFN}_{2}: \mathrm{C}, 46.72 ; \mathrm{H}$, 3.92; N, 10.90. Found: C, 46.64; H, 4.10; N, 10.93 .

\section{8-Bromo- $N$-(tert $H, 6 H$-pyrimido[1,2-c][1,3]benzothiazin-6-imine}

$(24 k)$. Using the general procedure as described for 22e, compound 20k $(257.1 \mathrm{mg}$, $1.00 \mathrm{mmol}$ ) was allowed to react at rt overnight. Purification by flash chromatography over aluminum oxide with $n$-hexane-EtOAc (1:0 to 9:1) gave the title compound $24 \mathbf{k}$ as colorless solid ( $335.3 \mathrm{mg}, 95 \%$ ): $\mathrm{mp} 89{ }^{\circ} \mathrm{C}$ (from $n$-hexane); IR (neat) $\mathrm{cm}^{-1}: 1595(\mathrm{C}=\mathrm{N}) ;{ }^{1} \mathrm{H}-\mathrm{NMR}\left(500 \mathrm{MHz}, \mathrm{CDCl}_{3}\right) \delta: 1.42\left(9 \mathrm{H}, \mathrm{s}, 3 \times \mathrm{CH}_{3}\right)$, $1.87-1.92\left(2 \mathrm{H}, \mathrm{m}, \mathrm{CH}_{2}\right), 3.62\left(2 \mathrm{H}, \mathrm{t}, J=5.4 \mathrm{~Hz}, \mathrm{CH}_{2}\right), 3.86(2 \mathrm{H}, \mathrm{t}, J=6.0 \mathrm{~Hz}$, $\left.\mathrm{CH}_{2}\right), 7.07(1 \mathrm{H}, \mathrm{dd}, J=8.0,7.4 \mathrm{~Hz}, \mathrm{Ar}), 7.55(1 \mathrm{H}, \mathrm{d}, J=7.4 \mathrm{~Hz}, \mathrm{Ar}), 8.19(1 \mathrm{H}, \mathrm{d}$, $J=8.0 \mathrm{~Hz}, \mathrm{Ar}) ;{ }^{13} \mathrm{C}-\mathrm{NMR}\left(125 \mathrm{MHz}, \mathrm{CDCl}_{3}\right) \delta: 21.7,30.1(3 \mathrm{C}), 45.3,45.3,54.3$, $118.6,126.5,127.5,129.9,130.7,133.8,137.4,147.5$; Anal. calcd for $\mathrm{C}_{15} \mathrm{H}_{18} \mathrm{BrN}_{3} \mathrm{~S}$ : C, 51.14; H, 5.15; N, 11.93. Found: C, 50.89; H, 5.06; N, 11.83 .

Compound 27k. Using the general procedure as described for $25 \mathbf{a}$, compound $24 \mathbf{k}(52.8 \mathrm{mg}, 0.15 \mathrm{mmol})$ was allowed to react for $2 \mathrm{~h}$ with TFA $(1.5 \mathrm{~mL})$ and MS4 $\AA$ ( $225 \mathrm{mg})$. Purification by flash chromatography over silica gel with $n$ hexane-EtOAc (2:1) gave the title compound $27 \mathbf{k}$ as colorless solid (31.6 mg, $71 \%$ ): $\mathrm{mp} 138-139{ }^{\circ} \mathrm{C}$ (from $\mathrm{CHCl}_{3}-n$-hexane); IR (neat) $\mathrm{cm}^{-1}: 1567(\mathrm{C}=\mathrm{N}) ;{ }^{1} \mathrm{H}-$ NMR $\left(500 \mathrm{MHz}, \mathrm{CDCl}_{3}\right) \delta: 1.94-1.98\left(2 \mathrm{H}, \mathrm{m}, \mathrm{CH}_{2}\right), 3.69(2 \mathrm{H}, \mathrm{t}, J=5.7 \mathrm{~Hz}$, $\left.\mathrm{CH}_{2}\right), 4.02\left(2 \mathrm{H}, \mathrm{t}, J=6.0 \mathrm{~Hz}, \mathrm{CH}_{2}\right), 7.10(1 \mathrm{H}, \mathrm{dd}, J=8.3,7.7 \mathrm{~Hz}, \mathrm{Ar}), 7.33(1 \mathrm{H}$, br s, NH), $7.56(1 \mathrm{H}, \mathrm{dd}, J=7.7,1.4 \mathrm{~Hz}, \mathrm{Ar}), 8.23(1 \mathrm{H}, \mathrm{dd}, J=8.3,1.4 \mathrm{~Hz}, \mathrm{Ar})$; ${ }^{13} \mathrm{C}-\mathrm{NMR}\left(125 \mathrm{MHz}, \mathrm{CDCl}_{3}\right) \delta: 20.8,43.7,45.0,117.6,126.8,127.9,128.8$, 130.5, 134.1, 146.2, 152.7; Anal. calcd for $\mathrm{C}_{11} \mathrm{H}_{10} \mathrm{BrN}_{3} \mathrm{~S}: \mathrm{C}, 44.61 ; \mathrm{H}, 3.40 ; \mathrm{N}$, 14.19. Found: C, 44.36; H, 3.64; N, 13.96. 


\subsubsection{Synthesis of 3,4-Dihydro-2H,6H-pyrimido[1,2-c] naphtho[2,3-e][1,3]thiazin-6-imine (27r)}

2-(3-Fluoronaphthalen-2-yl)-1,4,5,6-tetrahydropyrimidine (20r). 1-Fluoro-2naphthaldehyde $17 \mathbf{r}(0.96 \mathrm{~g}, 5.52 \mathrm{mmol})$ was subjected to the general procedure as described for 18j to give the title compound $\mathbf{2 0 r}$ as pale yellow crystals $(0.85 \mathrm{~g}$, $67 \%$ ): mp 128-130 ${ }^{\circ} \mathrm{C}$ (from $\mathrm{CHCl}_{3}-\mathrm{EtOAc}_{-} \mathrm{Et}_{2} \mathrm{O}$ ); IR (neat) $\mathrm{cm}^{-1}: 1619(\mathrm{C}=\mathrm{N}$ ); ${ }^{1} \mathrm{H}-\mathrm{NMR}\left(500 \mathrm{MHz}, \mathrm{CDCl}_{3}\right) \delta: 1.86-1.91\left(2 \mathrm{H}, \mathrm{m}, \mathrm{CH}_{2}\right), 3.52(4 \mathrm{H}, \mathrm{t}, J=5.7 \mathrm{~Hz}$, $\left.2 \times \mathrm{CH}_{2}\right), 5.21(1 \mathrm{H}$, br s, NH), 7.41-7.44 $(2 \mathrm{H}, \mathrm{m}, \mathrm{Ar}), 7.49(1 \mathrm{H}, \mathrm{t}, J=7.4 \mathrm{~Hz}$, Ar), $7.73(1 \mathrm{H}, \mathrm{d}, J=8.6 \mathrm{~Hz}, \mathrm{Ar}), 7.83(1 \mathrm{H}, \mathrm{d}, J=8.6 \mathrm{~Hz}, \mathrm{Ar}), 8.27(1 \mathrm{H}, \mathrm{d}$, $J=8.0 \mathrm{~Hz}, \mathrm{Ar}) ;{ }^{13} \mathrm{C}-\mathrm{NMR}\left(125 \mathrm{MHz}, \mathrm{CDCl}_{3}\right) \delta: 20.5,42.2$ (2C), 111.7 (d, $J=22.8 \mathrm{~Hz}), \quad 124.5(\mathrm{~d}, \quad J=16.8 \mathrm{~Hz}), 125.6 \quad(\mathrm{~d}, \quad J=2.4 \mathrm{~Hz}), 126.7 \quad(\mathrm{~d}$, $J=4.8 \mathrm{~Hz}), 127.5,128.6,130.1,130.9(\mathrm{~d}, J=4.8 \mathrm{~Hz}), 134.2(\mathrm{~d}, J=9.6 \mathrm{~Hz})$, 151.9, $157.8(\mathrm{~d}, J=247.1 \mathrm{~Hz}) ;{ }^{19} \mathrm{~F}-\mathrm{NMR}\left(500 \mathrm{MHz}, \mathrm{CDCl}_{3}\right) \delta:-119.8$; HRMS (FAB) $m / z$ calcd for $\mathrm{C}_{14} \mathrm{H}_{14} \mathrm{FN}_{2}[\mathrm{M}+\mathrm{H}]^{+} 229.1141$; found: 229.1143 .

$N$-(tert-Butyl)-3,4-dihydro-2H,6H-pyrimido[1,2-c ]naphtho[2,3-e $][1,3]$ thiazin-6-imine (24r). Using the general procedure as described for 22e, compound $20 \mathrm{r}(228.3 \mathrm{mg}, 1.00 \mathrm{mmol})$ was allowed to react at $\mathrm{rt}$ overnight. Purification by flash chromatography over silica gel with $n$-hexane-EtOAc (1:1) gave the title compound $\mathbf{2 4 r}$ as colorless solid $(284.8 \mathrm{mg}, 88 \%): \mathrm{mp}$ 82.5-83.5 ${ }^{\circ} \mathrm{C}$, IR (neat) $\mathrm{cm}^{-1}: 1594(\mathrm{C}=\mathrm{N}) ;{ }^{1} \mathrm{H}-\mathrm{NMR}\left(400 \mathrm{MHz}, \mathrm{CDCl}_{3}\right) \delta$ : $1.42\left(9 \mathrm{H}, \mathrm{s}, 3 \times \mathrm{CH}_{3}\right), 1.92-1.98\left(2 \mathrm{H}, \mathrm{m}, \mathrm{CH}_{2}\right), 3.69(2 \mathrm{H}, \mathrm{t}, J=5.6 \mathrm{~Hz}$, $\left.\mathrm{CH}_{2}\right), 3.91\left(2 \mathrm{H}, \mathrm{t}, J=6.2 \mathrm{~Hz}, \mathrm{CH}_{2}\right), 7.38-7.43(1 \mathrm{H}, \mathrm{m}, \mathrm{Ar}), 7.45-7.49(1 \mathrm{H}$, $\mathrm{m}, \mathrm{Ar}), 7.60(1 \mathrm{H}, \mathrm{s}, \mathrm{Ar}), 7.69(1 \mathrm{H}, \mathrm{d}, J=7.8 \mathrm{~Hz}, \mathrm{Ar}), 7.87(1 \mathrm{H}, \mathrm{d}$, $J=8.0 \mathrm{~Hz}, \mathrm{Ar}), 8.70(1 \mathrm{H}, \mathrm{s}, \mathrm{Ar}) ;{ }^{13} \mathrm{C}-\mathrm{NMR}\left(100 \mathrm{MHz}, \mathrm{CDCl}_{3}\right) \delta: 22.0$, 30.1 (3C), 45.3, 45.5, 54.3, 122.5, 125.8, 125.9, 126.3, 126.5, 127.8, 128.5, $129.2,131.7,133.9,138.4,148.5$; HRMS (FAB) $\mathrm{m} / z$ calcd for $\mathrm{C}_{19} \mathrm{H}_{22} \mathrm{~N}_{3} \mathrm{~S}$ $[\mathrm{M}+\mathrm{H}]^{+}$324.1534; found: 324.1526.

Compound 27r. Using the general procedure as described for 25a, compound 24r $(64.7 \mathrm{mg}, 0.2 \mathrm{mmol})$ was allowed to react for $2 \mathrm{~h}$. Purification by flash chromatography over aluminum oxide with $n$-hexane-EtOAc $(4: 1)$ gave the title compound $27 \mathbf{r}$ as colorless solid $\left(36.6 \mathrm{mg}, 68 \%\right.$ ): $\mathrm{mp} 180-181{ }^{\circ} \mathrm{C}$ (from $\mathrm{CHCl}_{3}-$ $n$-hexane); IR (neat) $\mathrm{cm}^{-1}: 1627(\mathrm{C}=\mathrm{N}), 1572(\mathrm{C}=\mathrm{N}) ;{ }^{1} \mathrm{H}-\mathrm{NMR}(400 \mathrm{MHz}$, $\left.\mathrm{CDCl}_{3}\right) \delta:$ 1.98-2.04 (2H, m, $\left.\mathrm{CH}_{2}\right), 3.75\left(2 \mathrm{H}, \mathrm{t}, J=5.5 \mathrm{~Hz}, \mathrm{CH}_{2}\right), 4.06(2 \mathrm{H}, \mathrm{t}$, $\left.J=6.2 \mathrm{~Hz}, \mathrm{CH}_{2}\right), 7.40-7.51(3 \mathrm{H}, \mathrm{m}, \mathrm{Ar}), 7.68(1 \mathrm{H}, \mathrm{d}, J=8.3 \mathrm{~Hz}, \mathrm{Ar}), 7.87(1 \mathrm{H}$, $\mathrm{d}, J=8.3 \mathrm{~Hz}, \mathrm{Ar}), 8.74(1 \mathrm{H}, \mathrm{s}, \mathrm{Ar}) ;{ }^{13} \mathrm{C}-\mathrm{NMR}\left(100 \mathrm{MHz}, \mathrm{CDCl}_{3}\right) \delta: 21.1,43.9$, 45.1, 121.6, 125.0, 125.4, 126.1, 126.3, 128.1, 129.2, 129.2, 131.6, 133.9, 147.1, 153.4; HRMS (FAB) $m / z$ calcd for $\mathrm{C}_{15} \mathrm{H}_{14} \mathrm{~N}_{3} \mathrm{~S}[\mathrm{M}+\mathrm{H}]^{+}$268.0908; found: 268.0909 . 


\subsubsection{Synthesis of 3,4-Dihydro-2H,6H-pyrimido[1,2-c] pyrido[3,2-e][1,3]thiazin-6-imine $(27 s)$}

Using general procedure as described for 25f, reaction of 2-(2-bromopyridin-3-yl)1,4,5,6-tetrahydropyrimidine $21 \mathrm{~s}(58.8 \mathrm{mg}, 0.25 \mathrm{mmol})$ and purification by flash chromatography over aluminum oxide with $n$-hexane-EtOAc $(7: 3)$ gave the title compound 27s as colorless solid (17.4 mg, $32 \%$ ): $\mathrm{mp} 181-183{ }^{\circ} \mathrm{C}$ (from $\mathrm{CHCl}_{3}$ $n$-hexane); IR (neat) $\mathrm{cm}^{-1}: 1624(\mathrm{C}=\mathrm{N}), 1582(\mathrm{C}=\mathrm{N}) ;{ }^{1} \mathrm{H}-\mathrm{NMR}(500 \mathrm{MHz}$, $\left.\mathrm{CDCl}_{3}\right) \delta: 1.96-2.01\left(2 \mathrm{H}, \mathrm{m}, \mathrm{CH}_{2}\right), 3.70\left(2 \mathrm{H}, \mathrm{t}, J=5.7 \mathrm{~Hz}, \mathrm{CH}_{2}\right), 4.05(2 \mathrm{H}, \mathrm{t}$, $\left.J=6.3 \mathrm{~Hz}, \mathrm{CH}_{2}\right), 7.17(1 \mathrm{H}, \mathrm{dd}, J=8.0,4.6 \mathrm{~Hz}, \mathrm{Ar}), 7.39(1 \mathrm{H}$, br s, NH), 8.46-8.50 (2H, m, Ar); ${ }^{13} \mathrm{C}-\mathrm{NMR}\left(125 \mathrm{MHz}, \mathrm{CDCl}_{3}\right) \delta: 20.8,43.8,45.2,121.4$, 123.7, 136.3, 145.3, 151.2, 151.3, 153.5; HRMS (FAB): $m / z$ calcd for $\mathrm{C}_{10} \mathrm{H}_{11} \mathrm{~N}_{4} \mathrm{~S}$ $[\mathrm{M}+\mathrm{H}]^{+}$219.0704; found: 219.0703 .

\subsubsection{Synthesis of 2,3-Dihydronaphtho[2,1-e]pyrimido [1,2-c][1,3]thiazin-12(1H)- imine (27t)}

2,3-Dihydronaphtho[2,1-e]pyrimido[1,2-c][1,3]thiazine-12(1H)-thione $21 \mathrm{t}$ (71.1 $\mathrm{mg}, 0.25 \mathrm{mmol}$ ) was subjected to the general procedure as described for $\mathbf{2 5 \mathbf { f }}$ to give the title compound $27 \mathrm{t}$ as colorless solid $\left(42.3 \mathrm{mg}, 63 \%\right.$ ): $\mathrm{mp} 157{ }^{\circ} \mathrm{C}$ (from $\mathrm{CHCl}_{3}-$ $n$-hexane); IR (neat) $\mathrm{cm}^{-1}$ : $1615(\mathrm{C}=\mathrm{N}), 1572(\mathrm{C}=\mathrm{N}) ;{ }^{1} \mathrm{H}-\mathrm{NMR}\left(500 \mathrm{MHz}, \mathrm{CDCl}_{3}\right)$ $\delta: 1.99-2.03\left(2 \mathrm{H}, \mathrm{m}, \mathrm{CH}_{2}\right), 3.75\left(2 \mathrm{H}, \mathrm{t}, J=5.4 \mathrm{~Hz}, \mathrm{CH}_{2}\right), 4.07(2 \mathrm{H}, \mathrm{t}, J=6.0 \mathrm{~Hz}$, $\left.\mathrm{CH}_{2}\right), 7.33(1 \mathrm{H}$, br s, NH), 7.53-7.57 $(2 \mathrm{H}, \mathrm{m}, \mathrm{Ar}), 7.66(1 \mathrm{H}, \mathrm{d}, J=8.6 \mathrm{~Hz}, \mathrm{Ar})$, $7.80-7.90(2 \mathrm{H}, \mathrm{m}, \mathrm{Ar}), 8.30(1 \mathrm{H}, \mathrm{d}, J=9.2 \mathrm{~Hz}, \mathrm{Ar}) ;{ }^{13} \mathrm{C}-\mathrm{NMR}\left(125 \mathrm{MHz}, \mathrm{CDCl}_{3}\right)$ $\delta: 20.9,43.7,45.1,123.2,124.0,125.1,125.9,126.7,126.7,127.5,127.8,128.5$, 133.9, 147.1, 152.7; HRMS (FAB): $m / z$ calcd for $\mathrm{C}_{15} \mathrm{H}_{14} \mathrm{~N}_{3} \mathrm{~S}[\mathrm{M}+\mathrm{H}]^{+} 268.0908$; found: 268.0906.

\subsubsection{Synthesis of 3,4-Dihydro-2H,6H-pyrimido[1,2-c] thieno[2,3-e][1,3]thiazin-6-imine (27u)}

2-(3-Bromothiophen-2-yl)-1,4,5,6-tetrahydropyrimidine (20u). 3-Bromothiophene-2-carbaldehyde $\mathbf{1 7 u}(1.29 \mathrm{~g}, 6.75 \mathrm{mmol})$ was subjected to the general procedure as described for $\mathbf{1 8 j}$ to give the title compound $\mathbf{2 0 u}$ as pale yellow crystals $(1.11 \mathrm{~g}, 67 \%)$ : $\mathrm{mp} 61-63{ }^{\circ} \mathrm{C}$ (from $\mathrm{CHCl}_{3}-n$-hexane); IR (neat) $\mathrm{cm}^{-1}$ : $1611(\mathrm{C}=\mathrm{N}) ;{ }^{1} \mathrm{H}-\mathrm{NMR}\left(400 \mathrm{MHz}, \mathrm{CDCl}_{3}\right) \delta: 1.83-1.88\left(2 \mathrm{H}, \mathrm{m}, \mathrm{CH}_{2}\right), 3.48(4 \mathrm{H}, \mathrm{t}$, $\left.J=5.9 \mathrm{~Hz}, 2 \times \mathrm{CH}_{2}\right), 6.07(1 \mathrm{H}$, br s, NH$), 6.92(1 \mathrm{H}, \mathrm{d}, J=5.4 \mathrm{~Hz}, \mathrm{Ar}), 7.24$ $(1 \mathrm{H}, \mathrm{d}, J=5.4 \mathrm{~Hz}, \mathrm{Ar}) ;{ }^{13} \mathrm{C}-\mathrm{NMR}\left(100 \mathrm{MHz}, \mathrm{CDCl}_{3}\right) \delta: 20.5,42.0$ (2C), 105.8, 127.3, 131.4, 135.6, 149.3; HRMS (FAB): $m / z$ calcd for $\mathrm{C}_{8} \mathrm{H}_{10} \mathrm{BrN}_{2} \mathrm{~S}[\mathrm{M}+\mathrm{H}]^{+}$ 244.9748; found: 244.9742 . 
3,4-Dihydro-2H,6H-pyrimido[1,2-c]thieno[2,3-e][1,3] thiazin-6-thione (21u). To a mixture of $20 \mathrm{u}(122.6 \mathrm{mg}, 0.50 \mathrm{mmol})$ and $\mathrm{NaH}(40.0 \mathrm{mg}, 1.0 \mathrm{mmol} ; 60 \%$ oil suspension) in DMF $(1.7 \mathrm{~mL})$ was added $\mathrm{CS}_{2}(0.060 \mathrm{~mL}, 1.0 \mathrm{mmol})$ under an Ar atmosphere. After being stirred at $80{ }^{\circ} \mathrm{C}$ for $12 \mathrm{~h}$, the mixture was concentrated. The residue was purified by flash chromatography over silica gel with $n$ hexane-EtOAc $(8: 2)$ to give the title compound $\mathbf{2 1 u}$ as pale yellow solid $(80.5 \mathrm{mg}$, $67 \%$ ): $\mathrm{mp} 167{ }^{\circ} \mathrm{C}$ (from $\mathrm{CHCl}_{3}-n$-hexane); IR (neat) $\mathrm{cm}^{-1}: 1624(\mathrm{C}=\mathrm{N}) ;{ }^{13} \mathrm{C}$ $\mathrm{NMR}\left(400 \mathrm{MHz}, \mathrm{CDCl}_{3}\right) \delta: 2.04-2.10\left(2 \mathrm{H}, \mathrm{m}, \mathrm{CH}_{2}\right), 3.68(2 \mathrm{H}, \mathrm{t}, J=5.5 \mathrm{~Hz}$, $\left.\mathrm{CH}_{2}\right), 4.42\left(2 \mathrm{H}, \mathrm{t}, J=6.1 \mathrm{~Hz}, \mathrm{CH}_{2}\right), 6.76(1 \mathrm{H}, \mathrm{d}, J=5.4 \mathrm{~Hz}, \mathrm{Ar}), 7.49(1 \mathrm{H}, \mathrm{d}$, $J=5.4 \mathrm{~Hz}, \mathrm{Ar}) ;{ }^{13} \mathrm{C}-\mathrm{NMR}\left(100 \mathrm{MHz}, \mathrm{CDCl}_{3}\right) \delta: 21.5,45.0,48.5,122.3,128.4$, 130.8, 131.0, 141.7, 189.7; HRMS (FAB): $m / z$ calcd for $\mathrm{C}_{9} \mathrm{H}_{9} \mathrm{~N}_{2} \mathrm{~S}_{3}[\mathrm{M}+\mathrm{H}]^{+}$ 240.9928; found: 240.9936.

Compound $27 \mathbf{u}$. Compound $21 \mathbf{u}(60.1 \mathrm{mg}, 0.25 \mathrm{mmol})$ was subjected to general procedure as described for $25 \mathrm{f}$ to give the title compound $\mathbf{2 7} \mathbf{u}$ as colorless solid (19.4 mg, $35 \%$ ): $\mathrm{mp} 100-101{ }^{\circ} \mathrm{C}$ (from $\mathrm{CHCl}_{3}-n$-hexane); IR (neat) $\mathrm{cm}^{-1}$ : $1616(\mathrm{C}=\mathrm{N}), 1567(\mathrm{C}=\mathrm{N}) ;{ }^{13} \mathrm{C}-\mathrm{NMR}\left(400 \mathrm{MHz}, \mathrm{CDCl}_{3}\right) \delta: 1.99-2.05(2 \mathrm{H}, \mathrm{m}$, $\left.\mathrm{CH}_{2}\right), 3.62\left(2 \mathrm{H}, \mathrm{t}, J=5.6 \mathrm{~Hz}, \mathrm{CH}_{2}\right), 3.99\left(2 \mathrm{H}, \mathrm{t}, J=6.1 \mathrm{~Hz}, \mathrm{CH}_{2}\right), 6.74(1 \mathrm{H}, \mathrm{d}$, $J=5.4 \mathrm{~Hz}, \mathrm{Ar}), 7.28\left(1 \mathrm{H}\right.$, br s, NH), $7.41(1 \mathrm{H}, \mathrm{d}, J=5.4 \mathrm{~Hz}, \mathrm{Ar}) ;{ }^{13} \mathrm{C}-\mathrm{NMR}$ $\left(100 \mathrm{MHz}, \mathrm{CDCl}_{3}\right) \delta: 21.2,43.5,44.5,123.3,125.9,127.0,129.9,143.7,153.7$; Anal. calcd for $\mathrm{C}_{9} \mathrm{H}_{9} \mathrm{~N}_{3} \mathrm{~S}_{2}$ : C, 48.40; H, 4.06; N, 18.82. Found: C, 48.38; H, 3.98; $\mathrm{N}, 18.75$.

\subsubsection{Synthesis of 3,4-Dihydro-9-(4- methoxycarbonylphenyl)-2H,6H-pyrimido [1,2-c][1,3]benzothiazin-6-imine (31a)}

$\mathrm{N}$-(tert-Butyl)-3,4-dihydro-9-(4-methoxycarbonylphenyl)-2H,6H-pyrimido [1, 2-c][1,3]benzothiazin-6-imine (29a). Using the general procedure as described for 221, $N$-(tert-butyl)-9-bromo-3,4-dihydro- $2 H, 6 H$-pyrimido[1,2-c][1,3]benzothiazin-6-imine $22 \mathrm{k}(52.8 \mathrm{mg}, 0.15 \mathrm{mmol})$ was allowed to react with 4(methoxycarbonyl)phenylboronic acid $(32.4 \mathrm{mg}, 0.18 \mathrm{mmol})$ for $1 \mathrm{~h}$. Purification by flash chromatography over aluminum oxide with $n$-hexane-EtOAc (1:0 to 9:1) gave the title compound 29a as colorless solid (47.3 mg, $77 \%$ ): mp 201-202 ${ }^{\circ} \mathrm{C}$ (from $\mathrm{CHCl}_{3}-n$-hexane); IR (neat) $\mathrm{cm}^{-1}$ : $1719(\mathrm{C}=\mathrm{O}), 1593(\mathrm{C}=\mathrm{N}) ;{ }^{1} \mathrm{H}-\mathrm{NMR}$ $\left(400 \mathrm{MHz}, \mathrm{CDCl}_{3}\right) \delta: 1.40\left(\mathrm{~s}, 9 \mathrm{H}, 3 \times \mathrm{CH}_{3}\right), 1.90-1.96\left(\mathrm{~m}, 2 \mathrm{H}, \mathrm{CH}_{2}\right), 3.65(\mathrm{t}$, $\left.J=5.5 \mathrm{~Hz}, 2 \mathrm{H}, \mathrm{CH}_{2}\right), 3.89\left(\mathrm{t}, J=6.1 \mathrm{~Hz}, 2 \mathrm{H}, \mathrm{CH}_{2}\right), 3.94\left(\mathrm{~s}, 3 \mathrm{H}, \mathrm{CH}_{3}\right), 7.36(\mathrm{~d}$, $J=1.7 \mathrm{~Hz}, 1 \mathrm{H}, \mathrm{Ar}), 7.44(\mathrm{dd}, J=8.5,1.7 \mathrm{~Hz}, 1 \mathrm{H}, \mathrm{Ar}), 7.65(\mathrm{~d}, J=8.2 \mathrm{~Hz}, 2 \mathrm{H}$, Ar), $8.10(\mathrm{~d}, J=8.2 \mathrm{~Hz}, 2 \mathrm{H}, \mathrm{Ar}), 8.28(\mathrm{~d}, J=8.5 \mathrm{~Hz}, 1 \mathrm{H}, \mathrm{Ar}) ;{ }^{13} \mathrm{C}-\mathrm{NMR}$ $\left(100 \mathrm{MHz}, \mathrm{CDCl}_{3}\right) \delta: 21.9,30.0$ (3C), 45.2, 45.4, 52.1, 54.2, 123.0, 124.8, 127.0 (2C), 127.3, 129.1, 129.6, 129.8, 130.2 (2C), 138.0, 141.7, 143.8, 147.5, 166.8; HRMS (FAB): $m / z$ calcd for $\mathrm{C}_{23} \mathrm{H}_{26} \mathrm{~N}_{3} \mathrm{O}_{2} \mathrm{~S}[\mathrm{M}+\mathrm{H}]^{+} 408.1746$; found: 408.1748 . 
Compound 31a. Using the general procedure as described for 25a, compound 29a (38.4 mg, $0.094 \mathrm{mmol}$ ) was allowed to react for $2 \mathrm{~h}$. Purification by flash chromatography over aluminum oxide with $n$-hexane-EtOAc $(9: 1$ to $1: 1)$ gave the title compound 31a as $(27.3 \mathrm{mg}, 83 \%)$ : $\mathrm{mp} 185-186{ }^{\circ} \mathrm{C}$ (from $\mathrm{CHCl}_{3}-n$-hexane); IR (neat) $\mathrm{cm}^{-1}$ : $1719(\mathrm{C}=\mathrm{O}), 1619(\mathrm{C}=\mathrm{N}), 1566(\mathrm{C}=\mathrm{N}) ;{ }^{1} \mathrm{H}-\mathrm{NMR}(500 \mathrm{MHz}$, $\left.\mathrm{CDCl}_{3}\right) \delta: 1.97-2.02\left(\mathrm{~m}, 2 \mathrm{H}, \mathrm{CH}_{2}\right), 3.71\left(\mathrm{t}, J=5.7 \mathrm{~Hz}, 2 \mathrm{H}, \mathrm{CH}_{2}\right), 3.94(\mathrm{~s}, 3 \mathrm{H}$, $\left.\mathrm{CH}_{3}\right), 4.04\left(\mathrm{t}, J=6.0 \mathrm{~Hz}, 2 \mathrm{H}, \mathrm{CH}_{2}\right), 7.27(\mathrm{~d}, J=1.7 \mathrm{~Hz}, 1 \mathrm{H}, \mathrm{Ar}), 7.46$ (dd, $J=8.0,1.7 \mathrm{~Hz}, 1 \mathrm{H}, \mathrm{Ar}), 7.63(\mathrm{~d}, J=8.6 \mathrm{~Hz}, 2 \mathrm{H}, \mathrm{Ar}), 8.10(\mathrm{~d}, J=8.6 \mathrm{~Hz}, 2 \mathrm{H}$, Ar), $8.30(\mathrm{~d}, J=8.0 \mathrm{~Hz}, 1 \mathrm{H}, \mathrm{Ar}) ;{ }^{13} \mathrm{C}-\mathrm{NMR}\left(125 \mathrm{MHz}, \mathrm{CDCl}_{3}\right) \delta: 21.0,43.8$, 45.0, 52.2, 122.0, 125.1, 126.2, 126.9 (2C), 129.5, 129.6, 129.7, 130.2 (2C), 142.1, 143.4, 146.2, 153.0, 166.7; HRMS (FAB): $m / z$ calcd for $\mathrm{C}_{19} \mathrm{H}_{18} \mathrm{~N}_{3} \mathrm{O}_{2} \mathrm{~S}[\mathrm{M}+\mathrm{H}]^{+}$ 352.1120; found: 352.1119 .

\subsubsection{Synthesis of 9-(4-Cyanophenyl)-3,4-dihydro-2H, 6H-pyrimido[1,2-c][1,3]benzothiazin-6-imine (31b)}

\section{$N$-(tert-Butyl)-9-(4-cyanophenyl)-3,4-dihydro-2H,6H-pyrimido[1,2-c][1,3]ben-} zothiazin-6-imine (29b). Using the general procedure as described for $\mathbf{2 2 l}$, $N$-(tert-butyl)-9-bromo-3,4-dihydro-2H,6 H-pyrimido[1,2-c][1,3]benzothiazin-6imine $22 \mathrm{k}$ ( $52.8 \mathrm{mg}, 0.15 \mathrm{mmol}$ ) was allowed to react with 4-cyanophenylboronic acid (26.4 mg, $0.18 \mathrm{mmol}$ ) for $1 \mathrm{~h}$. Purification by flash chromatography over aluminum oxide with $n$-hexane-EtOAc $(8: 2)$ gave the title compound $29 \mathbf{b}$ as colorless solid (53.9 mg, $96 \%$ ): $\mathrm{mp} 188-190{ }^{\circ} \mathrm{C}$ (from $\mathrm{CHCl}_{3}-n$-hexane); IR (neat) $\mathrm{cm}^{-1}: 2226(\mathrm{C} \equiv \mathrm{N}), 1593(\mathrm{C}=\mathrm{N}) ;{ }^{1} \mathrm{H}-\mathrm{NMR}\left(400 \mathrm{MHz}, \mathrm{CDCl}_{3}\right) \delta: 1.40(\mathrm{~s}$, 9H, $\left.3 \times \mathrm{CH}_{3}\right), 1.91-1.96\left(\mathrm{~m}, 2 \mathrm{H}, \mathrm{CH}_{2}\right), 3.65\left(\mathrm{t}, J=5.5 \mathrm{~Hz}, 2 \mathrm{H}, \mathrm{CH}_{2}\right), 3.89(\mathrm{t}$, $\left.J=6.1 \mathrm{~Hz}, 2 \mathrm{H}, \mathrm{CH}_{2}\right), 7.33(\mathrm{~d}, J=1.8 \mathrm{~Hz}, 1 \mathrm{H}, \mathrm{Ar}), 7.41(\mathrm{dd}, J=8.3,1.8 \mathrm{~Hz}$, 1H, Ar), 7.68 (d, J = 8.1 Hz, 2H, Ar), 7.73 (d, $J=8.1 \mathrm{~Hz}, 2 \mathrm{H}, A r), 8.29$ (d, $J=8.3 \mathrm{~Hz}, 1 \mathrm{H}, \mathrm{Ar}) ;{ }^{13} \mathrm{C}-\mathrm{NMR}\left(100 \mathrm{MHz}, \mathrm{CDCl}_{3}\right) \delta: 21.8,30.0$ (3C), 45.2, 45.4, 54.2, 111.7, 118.6, 123.0, 124.7, 127.6 (2C), 127.8, 129.3, 130.1, 132.6 (2C), 137.6, 140.7, 143.9, 147.4; HRMS (FAB): $\mathrm{m} / \mathrm{z}$ calcd for $\mathrm{C}_{22} \mathrm{H}_{23} \mathrm{~N}_{4} \mathrm{~S}[\mathrm{M}+\mathrm{H}]^{+}$ 375.1343; found: 375.1640 .

Compound 31b. Using the general procedure as described for 25a, compound 29b (32.5 mg, $0.087 \mathrm{mmol}$ ) was allowed to react for $2 \mathrm{~h}$. Purification by flash chromatography over aluminum oxide with $n$-hexane-EtOAc $(6: 4)$ gave the title compound 31b as colorless solid (20.8 mg, $75 \%$ ): $\mathrm{mp} 210-211^{\circ} \mathrm{C}$ (from $\mathrm{CHCl}_{3}-$ n-hexane); IR (neat) $\mathrm{cm}^{-1}: 2224(\mathrm{C} \equiv \mathrm{N}), 1615(\mathrm{C}=\mathrm{N}), 1577(\mathrm{C}=\mathrm{N}) ;{ }^{1} \mathrm{H}-\mathrm{NMR}$ $\left(400 \mathrm{MHz}, \mathrm{CDCl}_{3}\right) \delta: 1.97-2.02\left(\mathrm{~m}, 2 \mathrm{H}, \mathrm{CH}_{2}\right), 3.71\left(\mathrm{~d}, J=5.4 \mathrm{~Hz}, 2 \mathrm{H}, \mathrm{CH}_{2}\right)$, $4.04\left(\mathrm{t}, J=6.1 \mathrm{~Hz}, 2 \mathrm{H}, \mathrm{CH}_{2}\right), 7.24(\mathrm{~d}, J=1.7 \mathrm{~Hz}, 1 \mathrm{H}, \mathrm{Ar}), 7.43$ (dd, $J=8.3$, $1.7 \mathrm{~Hz}, 1 \mathrm{H}, \mathrm{Ar}), 7.67$ (d, $J=8.3 \mathrm{~Hz}, 2 \mathrm{H}, \mathrm{Ar}), 7.73$ (d, $J=8.3 \mathrm{~Hz}, 2 \mathrm{H}, \mathrm{Ar}), 8.32$ $(\mathrm{d}, J=8.3 \mathrm{~Hz}, 1 \mathrm{H}, \mathrm{Ar}) ;{ }^{13} \mathrm{C}-\mathrm{NMR}\left(100 \mathrm{MHz}, \mathrm{CDCl}_{3}\right) \delta: 21.0,43.8,45.0,111.9$, 118.5, 122.0, 125.0, 126.7, 127.6 (2C), 129.7, 129.9, 132.7 (2C), 141.2, 143.5, 146.0, 152.8; HRMS (FAB): $m / z$ calcd for $\mathrm{C}_{18} \mathrm{H}_{15} \mathrm{~N}_{4} \mathrm{~S}[\mathrm{M}+\mathrm{H}]^{+} 319.1017$; found: 319.1015 . 


\subsubsection{Synthesis of 3,4-Dihydro-9-(4-nitrophenyl)-2H, 6H-pyrimido[1,2-c][1,3]benzothiazin-6-imine (31c)}

$N$-(tert-Butyl)-3,4-dihydro-9-(4-nitrophenyl)-2H,6H-pyrimido[1,2-c][1,3]benzo thiazin-6-imine (29c). Using the general procedure as described for 22l, $\mathrm{N}$-(tertbutyl)-9-bromo-3,4-dihydro-2H,6H-pyrimido[1,2-c][1,3]benzothiazin-6-imine 22k (352.3 $\mathrm{mg}, 1.0 \mathrm{mmol}$ ) was allowed to react with 4-nitrophenylboronic acid (200.3 mg, $1.2 \mathrm{mmol}$ ) for $1 \mathrm{~h}$. Purification by flash chromatography over aluminum oxide with $n$-hexane-EtOAc $(9: 1$ to $2: 1)$ gave the title compound 29c as colorless solid (366.9 mg, $93 \%$ ): $\mathrm{mp} 201-202{ }^{\circ} \mathrm{C}$ (from $\mathrm{CHCl}_{3}-n$-hexane); IR (neat) $\mathrm{cm}^{-1}: 1590(\mathrm{C}=\mathrm{N}), 1514\left(\mathrm{NO}_{2}\right) ;{ }^{1} \mathrm{H}-\mathrm{NMR}\left(400 \mathrm{MHz}, \mathrm{CDCl}_{3}\right) \delta: 1.41(\mathrm{~s}$, $\left.9 \mathrm{H}, 3 \times \mathrm{CH}_{3}\right), 1.91-1.97\left(\mathrm{~m}, 2 \mathrm{H}, \mathrm{CH}_{2}\right), 3.65\left(\mathrm{t}, J=5.6 \mathrm{~Hz}, 2 \mathrm{H}, \mathrm{CH}_{2}\right), 3.90(\mathrm{t}$, $\left.J=6.1 \mathrm{~Hz}, 2 \mathrm{H}, \mathrm{CH}_{2}\right), 7.37(\mathrm{~d}, J=1.8 \mathrm{~Hz}, 1 \mathrm{H}, \mathrm{Ar}), 7.44(\mathrm{dd}, J=8.4,1.8 \mathrm{~Hz}$, $1 \mathrm{H}, \mathrm{Ar}), 7.74(\mathrm{~d}, J=8.5 \mathrm{~Hz}, 2 \mathrm{H}, \mathrm{Ar}), 8.28-8.32(\mathrm{~m}, 3 \mathrm{H}, \mathrm{Ar}) ;{ }^{13} \mathrm{C}-\mathrm{NMR}$ $\left(100 \mathrm{MHz}, \mathrm{CDCl}_{3}\right) \delta: 21.8,30.0$ (3C), 45.2, 45.5, 54.3, 123.2, 124.1 (2C), 124.8, 127.8 (2C), 128.0, 129.3, 130.2, 137.5, 140.4, 145.8, 147.4, 147.5; HRMS (FAB): $m / z$ calcd for $\mathrm{C}_{21} \mathrm{H}_{23} \mathrm{~N}_{4} \mathrm{O}_{2} \mathrm{~S}[\mathrm{M}+\mathrm{H}]^{+} 395.1542$; found: 395.1539 .

Compound 31c. Using the general procedure as described for $\mathbf{2 5 a}$, compound 29c $(77.0 \mathrm{mg}, 0.075 \mathrm{mmol})$ was allowed to react for $4 \mathrm{~h}$. Purification by flash chromatography over aluminum oxide with $n$-hexane-EtOAc (7:3) gave the title compound 31c as colorless solid $\left(50.7 \mathrm{mg}, 75 \%\right.$ ): $\mathrm{mp} 207-209{ }^{\circ} \mathrm{C}$ (from $\mathrm{CHCl}_{3}-$ $n$-hexane); IR (neat) $\mathrm{cm}^{-1}: 1615(\mathrm{C}=\mathrm{N}), 1598\left(\mathrm{NO}_{2}\right), 1573(\mathrm{C}=\mathrm{N}), 1508\left(\mathrm{NO}_{2}\right)$; ${ }^{1} \mathrm{H}-\mathrm{NMR}\left(500 \mathrm{MHz}, \mathrm{CDCl}_{3}\right) \delta: 1.98-2.02\left(\mathrm{~m}, 2 \mathrm{H}, \mathrm{CH}_{2}\right), 3.72(\mathrm{t}, J=5.7 \mathrm{~Hz}, 2 \mathrm{H}$, $\left.\mathrm{CH}_{2}\right), 4.05\left(\mathrm{t}, J=6.0 \mathrm{~Hz}, 2 \mathrm{H}, \mathrm{CH}_{2}\right), 7.27(\mathrm{~s}, 1 \mathrm{H}, \mathrm{Ar}), 7.46(\mathrm{~d}, J=8.0 \mathrm{~Hz}, 1 \mathrm{H}$, Ar), $7.72(\mathrm{~d}, J=8.6 \mathrm{~Hz}, 2 \mathrm{H}, \operatorname{Ar}), 8.30(\mathrm{~d}, J=8.6 \mathrm{~Hz}, 2 \mathrm{H}, \mathrm{Ar}), 8.34$ (d, $J=8.0 \mathrm{~Hz}, 1 \mathrm{H}, \mathrm{Ar}) ;{ }^{13} \mathrm{C}-\mathrm{NMR}\left(100 \mathrm{MHz}, \mathrm{CDCl}_{3}\right) \delta: 21.0,43.8,45.0,122.2$, 124.2 (2C), 125.1, 126.9, 127.8 (2C), 129.7, 130.0, 140.8, 145.4, 146.0, 147.6, 152.7; HRMS (FAB): $m / z$ calcd for $\mathrm{C}_{17} \mathrm{H}_{15} \mathrm{~N}_{4} \mathrm{O}_{2} \mathrm{~S}[\mathrm{M}+\mathrm{H}]^{+} 339.0916$; found: 339.0912 .

\subsubsection{Synthesis of 3,4-Dihydro-9-(4-trifluoromethylphenyl)- 2H,6H-pyrimido[1,2-c][1,3]benzothiazin-6-imine (31d)}

$\mathrm{N}$-(tert-Butyl)-3,4-dihydro-9-(4-trifluoromethylphenyl)-2H,6H-pyrimido[1,2-c] [1,3]benzothiazin-6-imine (29d). Using the general procedure as described for 22l, $\mathrm{N}$-(tert-butyl)-9-bromo-3,4-dihydro-2H,6H-pyrimido[1,2-c][1,3]benzothiazin6-imine $22 \mathrm{k}(52.8 \mathrm{mg}, 0.15 \mathrm{mmol})$ was allowed to react with 4-(trifluoromethyl)phenylboronic acid $(27.4 \mathrm{mg}, 0.18 \mathrm{mmol})$ for $1 \mathrm{~h}$. Purification by flash chromatography over aluminum oxide with $n$-hexane-EtOAc (1:0 to 9:1) gave the title compound 29d as colorless solid (51.9 mg, $83 \%$ ): $\mathrm{mp} 177-179{ }^{\circ} \mathrm{C}$ (from $n$ hexane); IR (neat) $\mathrm{cm}^{-1}: 1594(\mathrm{C}=\mathrm{N}) ;{ }^{1} \mathrm{H}-\mathrm{NMR}\left(400 \mathrm{MHz}, \mathrm{CDCl}_{3}\right) \delta: 1.40(\mathrm{~s}, 9 \mathrm{H}$, 
$\left.3 \times \mathrm{CH}_{3}\right), 1.90-1.96\left(\mathrm{~m}, 2 \mathrm{H}, \mathrm{CH}_{2}\right), 3.64\left(\mathrm{t}, J=5.6 \mathrm{~Hz}, 2 \mathrm{H}, \mathrm{CH}_{2}\right), 3.89(\mathrm{t}$, $\left.J=6.1 \mathrm{~Hz}, 2 \mathrm{H}, \mathrm{CH}_{2}\right), 7.33(\mathrm{~d}, J=2.0 \mathrm{~Hz}, 1 \mathrm{H}, \mathrm{Ar}), 7.41(\mathrm{dd}, J=8.3,2.0 \mathrm{~Hz}$, $1 \mathrm{H}, \mathrm{Ar}), 7.68$ (s, 4H, Ar), 8.29 (d, $J=8.3 \mathrm{~Hz}, 1 \mathrm{H}, \mathrm{Ar}) ;{ }^{13} \mathrm{C}-\mathrm{NMR}(125 \mathrm{MHz}$, $\left.\mathrm{CDCl}_{3}\right) \delta: 21.8,29.9(3 \mathrm{C}), 45.1,45.4,54.2,122.9,124.1(\mathrm{q}, J=271.1 \mathrm{~Hz}), 124.8$, $125.8(\mathrm{q}, \quad J=3.6 \mathrm{~Hz}, \quad 2 \mathrm{C}), 127.3$ (2C), 127.4, 129.2, 129.9, 130.0 (q, $J=32.8 \mathrm{~Hz}), 137.8,141.3,142.9,147.5 ;{ }^{19} \mathrm{~F}-\mathrm{NMR}\left(500 \mathrm{MHz}, \mathrm{CDCl}_{3}\right) \delta:-63.0$; HRMS (FAB): $m / z$ calcd for $\mathrm{C}_{22} \mathrm{H}_{23} \mathrm{~F}_{3} \mathrm{~N}_{3} \mathrm{~S}[\mathrm{M}+\mathrm{H}]^{+} 418.1565$; found: 418.1563 .

Compound 31d. Using the general procedure as described for 25a, compound 29d $(41.2 \mathrm{mg}, 1.0 \mathrm{mmol})$ was allowed to react for $4 \mathrm{~h}$ with TFA $(1.0 \mathrm{~mL})$ and MS4A (150 mg). Purification by flash chromatography over aluminum oxide with $n$-hexane-EtOAc (7:3) gave the title compound 31d as colorless solid (26.0 $\mathrm{mg}$, $73 \%$ ): $\mathrm{mp} 142-143{ }^{\circ} \mathrm{C}$ (from $\mathrm{CHCl}_{3}-n$-hexane); IR (neat) $\mathrm{cm}^{-1}: 1619(\mathrm{C}=\mathrm{N})$, $1567(\mathrm{C}=\mathrm{N}) ;{ }^{1} \mathrm{H}-\mathrm{NMR}\left(500 \mathrm{MHz}, \mathrm{CDCl}_{3}\right) \delta: 1.95-2.00\left(\mathrm{~m}, 2 \mathrm{H}, \mathrm{CH}_{2}\right), 3.70(\mathrm{t}$, $\left.J=5.4 \mathrm{~Hz}, 2 \mathrm{H}, \mathrm{CH}_{2}\right), 4.03\left(\mathrm{t}, J=6.0 \mathrm{~Hz}, 2 \mathrm{H}, \mathrm{CH}_{2}\right), 7.22(\mathrm{~s}, 1 \mathrm{H}, \mathrm{Ar}), 7.42(\mathrm{~d}$, $J=8.0 \mathrm{~Hz}, 1 \mathrm{H}, \mathrm{Ar}), 7.64(\mathrm{~d}, J=8.0 \mathrm{~Hz}, 2 \mathrm{H}, \mathrm{Ar}), 7.68(\mathrm{~d}, J=8.0 \mathrm{~Hz}, 2 \mathrm{H}, \mathrm{Ar})$, $8.30(\mathrm{~d}, J=8.0 \mathrm{~Hz}, 1 \mathrm{H}, \mathrm{Ar}) ;{ }^{13} \mathrm{C}-\mathrm{NMR}\left(125 \mathrm{MHz}, \mathrm{CDCl}_{3}\right) \delta: 20.9,43.8,44.9$, $121.9,124.0(\mathrm{q}, J=272.2 \mathrm{~Hz}, 125.0,125.7(\mathrm{t}, J=3.6 \mathrm{~Hz}, 2 \mathrm{C}), 126.2,127.2$ (2C), 129.5, 129.6, $130.1(\mathrm{q}, J=32.4 \mathrm{~Hz}), 141.7,142.5,146.1,152.8 ;{ }^{19} \mathrm{~F}-\mathrm{NMR}$ $\left(500 \mathrm{MHz}, \mathrm{CDCl}_{3}\right) \delta:-63.1 ;$ Anal. calcd for $\mathrm{C}_{18} \mathrm{H}_{14} \mathrm{~F}_{3} \mathrm{~N}_{3} \mathrm{~S}: \mathrm{C}, 59.82 ; \mathrm{H}, 3.90 ; \mathrm{N}$, 11.63. Found: C, 59.56; H, 3.81; N, 11.48 .

\subsubsection{Synthesis of 9-(4-Aminocarbonylphenyl)-3,4-dihydro- 2H,6H-pyrimido[1,2-c][1,3]benzothiazin-6- imine (31e)}

9-[(4-Aminocarbonyl)phenyl]- $\mathrm{N}$-(tert-butyl)-3,4-dihydro-2H,6H-pyrimido[1,2c][1,3]benzothiazin-6-imine (29e). Using the general procedure as described for 22l, $\mathrm{N}$-(tert-butyl)-9-bromo-3,4-dihydro- $2 \mathrm{H}, 6 \mathrm{H}$-pyrimido[1,2-c][1,3]benzothiazin6-imine $22 \mathbf{k}(52.8 \mathrm{mg}, 0.15 \mathrm{mmol})$ was allowed to react with 4-(aminocarbonyl)phenylboronic acid $(29.7 \mathrm{mg}, 0.18 \mathrm{mmol})$ in 1,4-dioxane for $1 \mathrm{~h}$. Purification by preparative TLC over aluminum oxide with $\mathrm{CHCl}_{3}-\mathrm{MeOH}(95: 5)$ gave the title compound 29e as colorless solid (31.2 mg, $53 \%$ ): $\mathrm{mp} 261-263{ }^{\circ} \mathrm{C}$ (from $\mathrm{MeOH}-\mathrm{CHCl}_{3}-n$-hexane); IR (neat) $\mathrm{cm}^{-1}$ : $1650(\mathrm{C}=\mathrm{O}), 1592(\mathrm{C}=\mathrm{N}) ;{ }^{1} \mathrm{H}-\mathrm{NMR}$ (400 MHz, $\left.\mathrm{CDCl}_{3}-\mathrm{CD}_{3} \mathrm{OD}\right) \delta: 1.41\left(\mathrm{~s}, 9 \mathrm{H}, 3 \times \mathrm{CH}_{3}\right), 1.92-1.98\left(\mathrm{~m}, 2 \mathrm{H}, \mathrm{CH}_{2}\right)$, $3.62\left(\mathrm{t}, J=5.5 \mathrm{~Hz}, 2 \mathrm{H}, \mathrm{CH}_{2}\right), 3.90\left(\mathrm{t}, J=6.1 \mathrm{~Hz}, 2 \mathrm{H}, \mathrm{CH}_{2}\right), 7.38(\mathrm{~d}, J=1.7 \mathrm{~Hz}$, $1 \mathrm{H}, \mathrm{Ar}), 7.47$ (dd, $J=8.5,1.7 \mathrm{~Hz}, 1 \mathrm{H}, \mathrm{Ar}), 7.66$ (d, $J=8.3 \mathrm{~Hz}, 2 \mathrm{H}, \mathrm{Ar}), 7.92$ (d, $J=8.3 \mathrm{~Hz}, 2 \mathrm{H}, \mathrm{Ar}), 8.17(\mathrm{~d}, J=8.5 \mathrm{~Hz}, 1 \mathrm{H}, \mathrm{Ar}) ;{ }^{13} \mathrm{C}-\mathrm{NMR}\left(100 \mathrm{MHz}, \mathrm{CDCl}_{3}-\right.$ $\left.\mathrm{CD}_{3} \mathrm{OD}\right) \delta: 21.6,29.7$ (3C), 44.7, 45.4, 54.2, 122.9, 124.8, 126.8, 126.9 (2C), 128.0 (2C), 128.8, 129.8, 132.6, 137.8, 141.8, 142.6, 148.7, 169.7; HRMS (FAB): $\mathrm{m} / z$ calcd for $\mathrm{C}_{22} \mathrm{H}_{25} \mathrm{~N}_{4} \mathrm{OS}[\mathrm{M}+\mathrm{H}]^{+}$393.1749; found: 393.1744 .

Compound 31e. TFA $(17 \mathrm{~mL})$ was added to a mixture of $29 \mathrm{e}(27.1 \mathrm{mg}$, $0.069 \mathrm{mmol}$ ) and MS4 $\AA$ (4.5 g, powder, activated by heating with Bunsen burner) 
in $\mathrm{CHCl}_{3}(3.0 \mathrm{~mL})$ and $\mathrm{MeOH}$ (10 drops). After being stirred under reflux for $9 \mathrm{~h}$, the mixture was concentrated. To a stirring mixture of this residue in $\mathrm{CHCl}_{3}$ was added dropwise $\mathrm{Et}_{3} \mathrm{~N}$ at $0{ }^{\circ} \mathrm{C}$ to adjust $\mathrm{pH}$ to $8-9$. The whole was extracted with EtOAc. The extract was washed with sat. $\mathrm{NaHCO}_{3}$, brine, and dried over $\mathrm{MgSO}_{4}$. After concentration, the residue was purified by preparative TLC over aluminum oxide with EtOAc-MeOH (95:5) to give compound 31e as colorless solid (13.0 mg, $56 \%$ ): $\mathrm{mp} 222-223{ }^{\circ} \mathrm{C}$ (from $\mathrm{MeOH}-\mathrm{CHCl}_{3}-n$-hexane); IR (neat) $\mathrm{cm}^{-1}: 1666(\mathrm{C}=\mathrm{O}), 1616(\mathrm{C}=\mathrm{N}), 1556(\mathrm{C}=\mathrm{N}) ;{ }^{1} \mathrm{H}-\mathrm{NMR}\left(400 \mathrm{MHz}, \mathrm{DMSO}-d_{6}\right) \delta$ : 1.83-1.89 (m, 2H, $\left.\mathrm{CH}_{2}\right), 3.59\left(\mathrm{t}, J=5.1 \mathrm{~Hz}, 2 \mathrm{H}, \mathrm{CH}_{2}\right), 3.92(\mathrm{t}, J=5.7 \mathrm{~Hz}, 2 \mathrm{H}$, $\left.\mathrm{CH}_{2}\right), 7.40(\mathrm{~s}, 1 \mathrm{H}, \mathrm{NH}), 7.60-7.62(\mathrm{~m}, 2 \mathrm{H}, \mathrm{Ar}), 7.81(\mathrm{~d}, J=8.3 \mathrm{~Hz}, 2 \mathrm{H}, \mathrm{Ar}), 7.96$ $(\mathrm{d}, J=8.3 \mathrm{~Hz}, 2 \mathrm{H}, \mathrm{Ar}), 8.04(\mathrm{~s}, 1 \mathrm{H}, \mathrm{NH}), 8.24$ (d, $J=8.3 \mathrm{~Hz}, 1 \mathrm{H}, \mathrm{Ar}), 8.74$ (s, $1 \mathrm{H}, \mathrm{NH}) ;{ }^{13} \mathrm{C}-\mathrm{NMR}\left(100 \mathrm{MHz}, \mathrm{DMSO}-d_{6}\right) \delta: 20.7,43.1,44.4,121.8,124.3$, 125.4, 126.5 (2C), 128.1 (2C), 129.0, 129.7, 133.8, 140.6, 141.1, 145.1, 149.5, 167.3; HRMS (FAB): $m / z$ calcd for $\mathrm{C}_{18} \mathrm{H}_{17} \mathrm{~N}_{4} \mathrm{OS}[\mathrm{M}+\mathrm{H}]^{+} 337.1123$; found: 337.1118 .

\subsubsection{Synthesis of 3,4-Dihydro-9-(4-methoxyphenyl)- 2H,6H-pyrimido[1,2-c][1,3]benzothiazin-6-imine (31f)}

$N$-(tert-Butyl)-3,4-dihydro-9-(4-methoxyphenyl)-2H,6H-pyrimido[1,2-c][1,3] benzothiazin-6-imine (29f). Using the general procedure as described for 221, $N$-(tert-butyl)-9-bromo-3,4-dihydro-2H,6H-pyrimido[1,2-c][1,3]benzothiazin-6imine $22 \mathrm{k}$ ( $52.8 \mathrm{mg}, 0.15 \mathrm{mmol})$ was allowed to react with 4-methoxyphenylboronic acid $(27.4 \mathrm{mg}, 0.18 \mathrm{mmol})$ for $1 \mathrm{~h}$. Purification by flash chromatography over aluminum oxide with $n$-hexane-EtOAc (1:0 to 9:1) gave the title compound 29f as colorless solid (54.7 mg, $96 \%$ ): mp 199-200 ${ }^{\circ} \mathrm{C}$ (from $\mathrm{CHCl}_{3}-n$-hexane); IR (neat) $\mathrm{cm}^{-1}: 1592(\mathrm{C}=\mathrm{N}) ;{ }^{1} \mathrm{H}-\mathrm{NMR}\left(400 \mathrm{MHz} \mathrm{CDCl}_{3}\right) \delta: 1.40(\mathrm{~s}, 9 \mathrm{H}, 3 \times$ $\left.\mathrm{CH}_{3}\right), 1.89-1.95\left(\mathrm{~m}, 2 \mathrm{H}, \mathrm{CH}_{2}\right), 3.63\left(\mathrm{t}, J=5.5 \mathrm{~Hz}, 2 \mathrm{H}, \mathrm{CH}_{2}\right), 3.84\left(\mathrm{~s}, 3 \mathrm{H}, \mathrm{CH}_{3}\right)$, $3.88\left(\mathrm{t}, J=6.0 \mathrm{~Hz}, 2 \mathrm{H}, \mathrm{CH}_{2}\right), 6.96(\mathrm{~d}, J=8.5 \mathrm{~Hz}, 2 \mathrm{H}, \mathrm{Ar}), 7.29(\mathrm{~d}, J=$ $2.0 \mathrm{~Hz}, 1 \mathrm{H}, \mathrm{Ar}), 7.38$ (dd, $J=8.3,2.0 \mathrm{~Hz}, 1 \mathrm{H}, \mathrm{Ar}), 7.53$ (d, $J=8.5 \mathrm{~Hz}, 2 \mathrm{H}, \mathrm{Ar})$, $8.22(\mathrm{~d}, J=8.3 \mathrm{~Hz}, 1 \mathrm{H}, \mathrm{Ar}) ;{ }^{13} \mathrm{C}-\mathrm{NMR}\left(100 \mathrm{MHz}, \mathrm{CDCl}_{3}\right) \delta: 21.9,30.0$ (3C), 45.1, 45.4, 54.1, 55.3, 114.3 (2C), 122.1, 124.4, 125.9, 128.1 (2C), 128.9, $129.4,131.9,138.4,142.5,147.8,159.8 ;$ HRMS (FAB): $\mathrm{m} / z$ calcd for $\mathrm{C}_{22} \mathrm{H}_{26} \mathrm{~N}_{3} \mathrm{OS}[\mathrm{M}+\mathrm{H}]^{+}$380.1797; found: 380.1801 .

Compound 31f. Using the general procedure as described for 25a, compound $29 f(28.4 \mathrm{mg}, 0.075 \mathrm{mmol})$ was allowed to react for $2 \mathrm{~h}$ with TFA (1.0 mL) and MS4A (150 mg). Purification by flash chromatography over aluminum oxide with $n$-hexane-EtOAc (3:1) gave the title compound 31f as colorless solid (20.0 $\mathrm{mg}$, $82 \%$ ): mp 93-94 ${ }^{\circ} \mathrm{C}$ (from $\mathrm{CHCl}_{3}-n$-hexane); IR (neat) $\mathrm{cm}^{-1}: 1620(\mathrm{C}=\mathrm{N}), 1567$ $(\mathrm{C}=\mathrm{N}) ;{ }^{1} \mathrm{H}-\mathrm{NMR}\left(400 \mathrm{MHz}, \mathrm{CDCl}_{3}\right) \delta: 1.96-2.02\left(\mathrm{~m}, 2 \mathrm{H}, \mathrm{CH}_{2}\right), 3.71(\mathrm{t}$, $\left.J=5.5 \mathrm{~Hz}, 2 \mathrm{H}, \mathrm{CH}_{2}\right), 3.85\left(\mathrm{~s}, 3 \mathrm{H}, \mathrm{CH}_{3}\right), 4.04\left(\mathrm{t}, J=6.2 \mathrm{~Hz}, 2 \mathrm{H}, \mathrm{CH}_{2}\right), 6.97(\mathrm{~d}$, $J=8.5 \mathrm{~Hz}, 2 \mathrm{H}, \mathrm{Ar}), 7.20(\mathrm{~d}, J=1.7 \mathrm{~Hz}, 1 \mathrm{H}, \mathrm{Ar}), 7.41(\mathrm{dd}, J=8.3,1.7 \mathrm{~Hz}, 1 \mathrm{H}$, 
Ar), $7.52(\mathrm{~d}, J=8.5 \mathrm{~Hz}, 2 \mathrm{H}, \mathrm{Ar}), 8.25(\mathrm{~d}, J=8.3 \mathrm{~Hz}, 1 \mathrm{H}, \mathrm{Ar}) ;{ }^{13} \mathrm{C}-\mathrm{NMR}$ $\left(100 \mathrm{MHz} \mathrm{CDCl}_{3}\right) \delta: 21.0,43.8,44.9,55.4,114.4$ (2C), 121.1, 124.7, 124.8, 128.1 (2C), 129.3, 129.3, 131.5, 143.1, 146.5, 153.5, 159.9; Anal. calcd for $\mathrm{C}_{18} \mathrm{H}_{17} \mathrm{~N}_{3} \mathrm{OS}$ : C, 66.85; H, 5.30; N, 12.99. Found: C, 66.95; H, 5.50; N, 12.89.

\subsubsection{Synthesis of 3,4-Dihydro-9-(4-methylthiophenyl)- 2H,6H-pyrimido[1,2-c][1,3]benzothiazin-6-imine (31g)}

$N$-(tert-Butyl)-3,4-dihydro-9-(4-methylthiophenyl)-2H,6H-pyrimido[1,2-c][1, 3]benzothiazin-6-imine $\mathbf{( 2 9 g )}$. Using the general procedure as described for $\mathbf{2 2 1}$, $\mathrm{N}$-(tert-butyl)-9-bromo-3,4-dihydro-2H,6H-pyrimido[1,2-c][1,3]benzothiazin-6imine 22k (52.8 mg, $0.15 \mathrm{mmol}$ ) was allowed to react with 4-(methylthio)phenylboronic acid $(30.2 \mathrm{mg}, 0.18 \mathrm{mmol})$ for $1 \mathrm{~h}$. Purification by flash chromatography over aluminum oxide with $n$-hexane-EtOAc (1:0 to 9:1) gave the title compound 29g as colorless solid (50.6 mg, $85 \%$ ): $\mathrm{mp} 201-202{ }^{\circ} \mathrm{C}$ (from $\mathrm{CHCl}_{3}-n$-hexane); IR (neat) $\mathrm{cm}^{-1}: 1592(\mathrm{C}=\mathrm{N}) ;{ }^{1} \mathrm{H}-\mathrm{NMR}\left(400 \mathrm{MHz}, \mathrm{CDCl}_{3}\right) \delta: 1.40(\mathrm{~s}, 9 \mathrm{H}$, $\left.3 \times \mathrm{CH}_{3}\right), 1.89-1.95\left(\mathrm{~m}, 2 \mathrm{H}, \mathrm{CH}_{2}\right), 2.51\left(\mathrm{~s}, 3 \mathrm{H}, \mathrm{CH}_{3}\right), 3.64(\mathrm{t}, J=5.5 \mathrm{~Hz}, 2 \mathrm{H}$, $\mathrm{CH}_{2}$ ), 3.88 (t, $J=6.1 \mathrm{~Hz}, 2 \mathrm{H}, \mathrm{CH}_{2}$ ), 7.30-7.32 (m, 3H, Ar), 7.40 (dd, $J=8.4$, $1.3 \mathrm{~Hz}, 1 \mathrm{H}, \mathrm{Ar}), 7.51$ (d, $J=8.3 \mathrm{~Hz}, 2 \mathrm{H}, \mathrm{Ar}), 8.23$ (d, $J=8.4 \mathrm{~Hz}, 1 \mathrm{H}, \mathrm{Ar} ;{ }^{13} \mathrm{C}-$ NMR (100 MHz, $\left.\mathrm{CDCl}_{3}\right) \delta: 15.7,21.9,30.0$ (3C), 45.1, 45.4, 54.2, 122.3, 124.4, $126.4,126.8$ (2C), 127.3 (2C), 129.0, 129.6, 136.0, 138.2, 138.8, 142.2, 147.7; HRMS (FAB): $m / z$ calcd for $\mathrm{C}_{22} \mathrm{H}_{26} \mathrm{~N}_{3} \mathrm{~S}_{2}[\mathrm{M}+\mathrm{H}]^{+} 396.1568$; found: 396.1566 .

Compound 31g. Using the general procedure as described for 25a, compound 29g (38.5 mg, $0.097 \mathrm{mmol}$ ) was allowed to react for $4 \mathrm{~h}$. Purification by flash chromatography over aluminum oxide with $n$-hexane-EtOAc $(9: 1$ to $7: 3)$ gave the title compound $\mathbf{3 1 g}$ as colorless solid (18.4 mg, $56 \%$ ): $\mathrm{mp} 151-153{ }^{\circ} \mathrm{C}$ (from $\mathrm{CHCl}_{3}-n$-hexane); IR (neat) $\mathrm{cm}^{-1}: 1620 \quad(\mathrm{C}=\mathrm{N}), 1573 \quad(\mathrm{C}=\mathrm{N}) ;{ }^{1} \mathrm{H}-\mathrm{NMR}$ $\left(400 \mathrm{MHz}, \mathrm{CDCl}_{3}\right) \delta: 1.96-2.02\left(\mathrm{~m}, 2 \mathrm{H}, \mathrm{CH}_{2}\right), 2.52\left(\mathrm{~s}, 3 \mathrm{H}, \mathrm{CH}_{3}\right), 3.71(\mathrm{t}$, $\left.J=5.5 \mathrm{~Hz}, 2 \mathrm{H}, \mathrm{CH}_{2}\right), 4.04\left(\mathrm{t}, J=6.1 \mathrm{~Hz}, 2 \mathrm{H}, \mathrm{CH}_{2}\right), 7.21(\mathrm{~d}, J=1.8 \mathrm{~Hz}, 1 \mathrm{H}$, Ar), $7.31(\mathrm{~d}, J=8.3 \mathrm{~Hz}, 2 \mathrm{H}, \mathrm{Ar}), 7.42(\mathrm{dd}, J=8.5,1.8 \mathrm{~Hz}, 1 \mathrm{H}, \mathrm{Ar}), 7.49$ (d, $J=8.3 \mathrm{~Hz}, 2 \mathrm{H}, \mathrm{Ar}), 8.27(\mathrm{~d}, J=8.5 \mathrm{~Hz}, 1 \mathrm{H}, \mathrm{Ar}) ;{ }^{13} \mathrm{C}-\mathrm{NMR}\left(100 \mathrm{MHz}, \mathrm{CDCl}_{3}\right)$ $\delta$ : 15.6, 21.0, 43.8, 44.9, 121.3, 124.7, 125.3, 126.7 (2C), 127.3 (2C), 129.4 (2C), 135.7, 139.2, 142.8, 146.5, 153.3; HRMS (FAB): $m / z$ calcd for $\mathrm{C}_{18} \mathrm{H}_{18} \mathrm{~N}_{3} \mathrm{~S}_{2}$ $[\mathrm{M}+\mathrm{H}]^{+}$340.0942; found: 340.0944 . 


\subsubsection{Synthesis of 3,4-Dihydro-9-(4-trifluomethoxyphenyl)- 2H,6H-pyrimido[1,2-c][1,3]benzothiazin-6-imine (31h)}

$\mathrm{N}$-(tert-Butyl)-3,4-dihydro-9-(4-trifluoromethoxyphenyl)-2H,6H-pyrimido [1, 2-c][1,3]benzothiazin-6-imine $\mathbf{( 2 9 h ) . ~ U s i n g ~ t h e ~ g e n e r a l ~ p r o c e d u r e ~ a s ~ d e s c r i b e d ~}$ for 221, $N$-(tert-butyl)-9-bromo-3,4-dihydro-2H,6H-pyrimido[1,2-c][1,3]benzothiazin-6-imine $22 \mathrm{k}(52.8 \mathrm{mg}, 0.15 \mathrm{mmol})$ was allowed to react with 4-(trifluoromethoxy)phenylboronic acid $(37.1 \mathrm{mg}, 0.18 \mathrm{mmol})$ for $1 \mathrm{~h}$. Purification by flash chromatography over aluminum oxide with $n$-hexane-EtOAc (1:0 to $9: 1)$ gave the title compound $\mathbf{2 9 h}$ as colorless solid $\left(59.7 \mathrm{mg}, 92 \%\right.$ ): $\mathrm{mp} 157{ }^{\circ} \mathrm{C}$ (from $\mathrm{CHCl}_{3}-n$-hexane); IR (neat) $\mathrm{cm}^{-1}: 1595(\mathrm{C}=\mathrm{N}) ;{ }^{1} \mathrm{H}-\mathrm{NMR}\left(500 \mathrm{MHz}, \mathrm{CDCl}_{3}\right) \delta$ : $1.40\left(\mathrm{~s}, 9 \mathrm{H}, 3 \times \mathrm{CH}_{3}\right), 1.91-1.95\left(\mathrm{~m}, 2 \mathrm{H}, \mathrm{CH}_{2}\right), 3.64\left(\mathrm{t}, J=5.4 \mathrm{~Hz}, 2 \mathrm{H}, \mathrm{CH}_{2}\right)$, $3.89\left(\mathrm{t}, J=6.0 \mathrm{~Hz}, 2 \mathrm{H}, \mathrm{CH}_{2}\right), 7.27-7.30(\mathrm{~m}, 3 \mathrm{H}, \mathrm{Ar}), 7.38(\mathrm{dd}, J=8.0,1.7 \mathrm{~Hz}$, $1 \mathrm{H}, \mathrm{Ar}), 7.59$ (d, $J=8.6 \mathrm{~Hz}, 2 \mathrm{H}, \mathrm{Ar}), 8.26(\mathrm{~d}, J=8.0 \mathrm{~Hz}, 1 \mathrm{H}, \mathrm{Ar}) ;{ }^{13} \mathrm{C}-\mathrm{NMR}$ $\left(100 \mathrm{MHz} \mathrm{CDCl}_{3}\right) \delta: 21.9,30.0(3 \mathrm{C}), 45.2,45.5,54.2,120.5$ (q, $\left.J=257.4 \mathrm{~Hz}\right)$, 121.3 (2C), 122.8, 124.7, 127.0, 128.5 (2C), 129.1, 129.8, 138.0, 138.2, 141.5, 147.6, $149.2\left(\mathrm{q}, J=1.7 \mathrm{~Hz}\right.$ ); ${ }^{19} \mathrm{~F}-\mathrm{NMR}\left(500 \mathrm{MHz}, \mathrm{CDCl}_{3}\right) \delta$ : -58.3 ; HRMS (FAB): $m / z$ calcd for $\mathrm{C}_{22} \mathrm{H}_{23} \mathrm{~F}_{3} \mathrm{~N}_{3} \mathrm{OS}[\mathrm{M}+\mathrm{H}]^{+} 434.1514$; found: 434.1512 .

Compound $31 \mathbf{h}$. Using the general procedure as described for $\mathbf{2 5 a}$, compound 29h (44.8 mg, $0.103 \mathrm{mmol}$ ) was allowed to react for $2 \mathrm{~h}$. Purification by flash chromatography over aluminum oxide with $n$-hexane-EtOAc $(9: 1-7: 3)$ gave the title compound $\mathbf{3 1 h}$ as colorless solid $\left(17.3 \mathrm{mg}, 45 \%\right.$ ): $\mathrm{mp} 120{ }^{\circ} \mathrm{C}$ (from $\mathrm{CHCl}_{3}-$ $n$-hexane); IR (neat) $\mathrm{cm}^{-1}: 1621(\mathrm{C}=\mathrm{N}), 1571(\mathrm{C}=\mathrm{N}) ;{ }^{1} \mathrm{H}-\mathrm{NMR}(500 \mathrm{MHz}$, $\left.\mathrm{CDCl}_{3}\right) \delta$ : $1.98-2.02\left(\mathrm{~m}, 2 \mathrm{H}, \mathrm{CH}_{2}\right), 3.72\left(\mathrm{t}, J=5.7 \mathrm{~Hz}, 2 \mathrm{H}, \mathrm{CH}_{2}\right), 4.04(\mathrm{t}$, $\left.J=6.3 \mathrm{~Hz}, 2 \mathrm{H}, \mathrm{CH}_{2}\right), 7.22(\mathrm{~d}, J=1.7 \mathrm{~Hz}, 1 \mathrm{H}, \mathrm{Ar}), 7.29(\mathrm{~d}, J=8.6 \mathrm{~Hz}, 2 \mathrm{H}, \mathrm{Ar})$, $7.41(\mathrm{dd}, J=8.0,1.7 \mathrm{~Hz}, 1 \mathrm{H}, \mathrm{Ar}), 7.59$ (d, $J=8.6 \mathrm{~Hz}, 2 \mathrm{H}, \mathrm{Ar}), 8.29$ (d, $J=8.6 \mathrm{~Hz}, 1 \mathrm{H}, \mathrm{Ar}) ;{ }^{13} \mathrm{C}-\mathrm{NMR}\left(125 \mathrm{MHz}, \mathrm{CDCl}_{3}\right) \delta: 20.9,43.8,44.9,120.4$ (q, $J=257.5 \mathrm{~Hz}), 121.3(2 \mathrm{C}), 121.7,124.9,125.8,128.4(2 \mathrm{C}), 129.4,129.5,137.8$, 141.9, 146.2, 149.2, 153.0; ${ }^{19} \mathrm{~F}-\mathrm{NMR}\left(500 \mathrm{MHz}^{\mathrm{CDCl}} \mathrm{CDC}_{3}\right) \delta$ : -58.4 ; HRMS (FAB): $m / z$ calcd for $\mathrm{C}_{18} \mathrm{H}_{15} \mathrm{~F}_{3} \mathrm{~N}_{3} \mathrm{OS}[\mathrm{M}+\mathrm{H}]^{+}$378.0888; found: 378.0888.

\subsubsection{Synthesis of 3,4-Dihydro-9- (3-methoxycarbonylphenyl)-2H,6H-pyrimido[1,2-c] [1,3]benzothiazin-6-imine (31i)}

$\mathrm{N}$-(-(tert-Butyl)-3,4-dihydro-9-(3-methoxycarbonylphenyl)-2H,6Hc][1,3]benzo thiazin-6-imine (29i). Using the general procedure as described for 221, $\mathrm{N}$-(tertbutyl)-9-bromo-3,4-dihydro- $2 \mathrm{H}, 6 \mathrm{H}$-pyrimido[1,2-c][1,3]benzothiazin-6-imine 22k (52.8 mg, $0.15 \mathrm{mmol}$ ) was allowed to react with 3-(methoxycarbonyl)phenylboronic acid $(32.4 \mathrm{mg}, 0.18 \mathrm{mmol})$ for $1 \mathrm{~h}$. Purification by flash chromatography over 
aluminum oxide with $n$-hexane-EtOAc (1:0 to 9:1) gave the title compound $29 \mathbf{i}$ as colorless solid (56.2 mg, $92 \%$ ): $\mathrm{mp} 116-117.5{ }^{\circ} \mathrm{C}$ (from $n$-hexane); IR (neat) cm ${ }^{-1}$ : $1723(\mathrm{C}=\mathrm{O}), 1592(\mathrm{C}=\mathrm{N}) ;{ }^{1} \mathrm{H}-\mathrm{NMR}\left(500 \mathrm{MHz}, \mathrm{CDCl}_{3}\right) \delta: 1.41\left(\mathrm{~s}, 9 \mathrm{H}, 3 \times \mathrm{CH}_{3}\right)$, 1.91-1.96 (m, 2H, CH$), 3.65\left(\mathrm{t}, J=5.4 \mathrm{~Hz}, 2 \mathrm{H}, \mathrm{CH}_{2}\right), 3.89(\mathrm{t}, J=6.0 \mathrm{~Hz}, 2 \mathrm{H}$, $\left.\mathrm{CH}_{2}\right), 3.95\left(\mathrm{~s}, 3 \mathrm{H}, \mathrm{CH}_{3}\right), 7.37(\mathrm{~d}, J=1.7 \mathrm{~Hz}, 1 \mathrm{H}, \mathrm{Ar}), 7.45(\mathrm{dd}, J=8.6,1.7 \mathrm{~Hz}$, $1 \mathrm{H}, \operatorname{Ar}), 7.51(\mathrm{t}, J=8.0 \mathrm{~Hz}, 1 \mathrm{H}, \operatorname{Ar}), 7.78(\mathrm{~d}, J=8.0 \mathrm{~Hz}, 1 \mathrm{H}, \operatorname{Ar}), 8.04(\mathrm{~d}$, $J=8.0 \mathrm{~Hz}, 1 \mathrm{H}, \mathrm{Ar}), 8.26-8.28$ (m, $2 \mathrm{H}, \mathrm{Ar}) ;{ }^{13} \mathrm{C}-\mathrm{NMR}\left(125 \mathrm{MHz}, \mathrm{CDCl}_{3}\right) \delta: 21.9$, 30.0 (3C), 45.1, 45.4, 52.2, 54.2, 122.8, 124.7, 127.0, 128.0, 129.0, 129.1 (2C), 129.7, 130.8, 131.3, 138.1, 139.7, 141.7, 147.6, 166.8; HRMS (FAB): $m / z$ calcd for $\mathrm{C}_{23} \mathrm{H}_{26} \mathrm{~N}_{3} \mathrm{O}_{2} \mathrm{~S}[\mathrm{M}+\mathrm{H}]^{+}$408.1746; found: 408.1741 .

Compound 31i. Using the general procedure as described for 25a, compound 29i (34.2 $\mathrm{mg}, 0.084 \mathrm{mmol}$ ) was allowed to react for $3 \mathrm{~h}$. Purification by flash chromatography over aluminum oxide with $n$-hexane-EtOAc $(7: 3)$ gave the title compound 31i as colorless solid $\left(22.8 \mathrm{mg}, 77 \%\right.$ ): $\mathrm{mp} 131{ }^{\circ} \mathrm{C}$ (from $\mathrm{CHCl}_{3}-n$ hexane); IR (neat) $\mathrm{cm}^{-1}$ : $1721(\mathrm{C}=\mathrm{O}), 1620(\mathrm{C}=\mathrm{N}), 1568(\mathrm{C}=\mathrm{N}) ;{ }^{1} \mathrm{H}-\mathrm{NMR}$ $\left(500 \mathrm{MHz} \mathrm{CDCl}_{3}\right) \delta: 1.97-2.02\left(\mathrm{~m}, 2 \mathrm{H}, \mathrm{CH}_{2}\right), 3.72\left(\mathrm{t}, J=5.4 \mathrm{~Hz}, 2 \mathrm{H}, \mathrm{CH}_{2}\right), 3.96$ $\left(\mathrm{s}, 3 \mathrm{H}, \mathrm{CH}_{3}\right), 4.04\left(\mathrm{t}, J=6.0 \mathrm{~Hz}, 2 \mathrm{H}, \mathrm{CH}_{2}\right), 7.28(\mathrm{~d}, J=1.4 \mathrm{~Hz}, 1 \mathrm{H}, \mathrm{Ar}), 7.47$ (dd, $J=8.6,1.4 \mathrm{~Hz}, 1 \mathrm{H}, \mathrm{Ar}), 7.52$ (t, $J=7.7 \mathrm{~Hz}, 1 \mathrm{H}, \mathrm{Ar}), 7.76(\mathrm{~d}, J=7.7 \mathrm{~Hz}$, $1 \mathrm{H}, \mathrm{Ar}), 8.05$ (d, $J=7.7 \mathrm{~Hz}, 1 \mathrm{H}, \mathrm{Ar}), 8.25$ (s, 1H, Ar), 8.30 (d, $J=8.6 \mathrm{~Hz}, 1 \mathrm{H}$, $\mathrm{Ar}) ;{ }^{13} \mathrm{C}-\mathrm{NMR}\left(125 \mathrm{MHz}, \mathrm{CDCl}_{3}\right) \delta: 21.0,43.8,45.0,52.3,121.8,125.0,125.9$, 128.1, 129.0, 129.2, 129.5, 129.5, 130.9, 131.3, 139.4, 142.3, 146.3, 153.1, 166.7; Anal. calcd for $\mathrm{C}_{19} \mathrm{H}_{17} \mathrm{~N}_{3} \mathrm{O}_{2} \mathrm{~S}$ : C, 64.94; H, 4.88; N, 11.96. Found: C, 64.83; H, $4.79 ; \mathrm{N}, 11.84$.

\subsubsection{Synthesis of 9-(3-Cyanophenyl)-3,4-dihydro-2H, 6H-pyrimido[1,2-c][1,3]benzothiazin-6-imine (31j)}

$N$-(tert-Butyl)-9-(3-cyanophenyl)-3,4-dihydro-2H,6H-pyrimido[1,2-c][1,3]benzothiazin-6-imine (29j). Using the general procedure as described for 22l, $\mathrm{N}$-(tertbutyl)-9-bromo-3,4-dihydro-2H,6H-pyrimido[1,2-c][1,3]benzothiazin-6-imine 22k (52.8 $\mathrm{mg}, 0.15 \mathrm{mmol}$ ) was allowed to react with 3-cyanophenylboronic acid ( $26.5 \mathrm{mg}, 0.18 \mathrm{mmol}$ ) for $1 \mathrm{~h}$. Purification by flash chromatography over aluminum oxide with $n$-hexane-EtOAc (85:5) gave the title compound $29 \mathbf{j}$ as colorless solid (48.4 mg, $86 \%$ ): mp $165-167{ }^{\circ} \mathrm{C}$ (from $\mathrm{CHCl}_{3}-n$-hexane); IR (neat) $\mathrm{cm}^{-1}: 2230$ $(\mathrm{C} \equiv \mathrm{N}), 1593(\mathrm{C}=\mathrm{N}) ;{ }^{1} \mathrm{H}-\mathrm{NMR}\left(500 \mathrm{MHz}, \mathrm{CDCl}_{3}\right) \delta: 1.40\left(\mathrm{~s}, 9 \mathrm{H}, 3 \times \mathrm{CH}_{3}\right)$, 1.91-1.96 (m, 2H, $\left.\mathrm{CH}_{2}\right), 3.65\left(\mathrm{t}, J=5.7 \mathrm{~Hz}, 2 \mathrm{H}, \mathrm{CH}_{2}\right), 3.89(\mathrm{t}, J=6.3 \mathrm{~Hz}, 2 \mathrm{H}$, $\left.\mathrm{CH}_{2}\right), 7.31(\mathrm{~d}, J=1.7 \mathrm{~Hz}, 1 \mathrm{H}, \mathrm{Ar}), 7.38(\mathrm{dd}, J=8.0,1.7 \mathrm{~Hz}, 1 \mathrm{H}, \mathrm{Ar}), 7.55$ (t, $J=7.7 \mathrm{~Hz}, 1 \mathrm{H}, \mathrm{Ar}), 7.65(\mathrm{dt}, J=7.7,1.7 \mathrm{~Hz}, 1 \mathrm{H}, \mathrm{Ar}), 7.81(\mathrm{dt}, J=7.7,1.7 \mathrm{~Hz}$, $1 \mathrm{H}, \mathrm{Ar}), 7.86(\mathrm{t}, J=1.7 \mathrm{~Hz}, 1 \mathrm{H}, \mathrm{Ar}), 8.29(\mathrm{~d}, J=8.0 \mathrm{~Hz}, 1 \mathrm{H}, \mathrm{Ar}) ;{ }^{13} \mathrm{C}-\mathrm{NMR}$ $\left(100 \mathrm{MHz}, \mathrm{CDCl}_{3}\right) \delta: 21.8,30.0$ (3C), 45.1, 45.4, 54.2, 113.2, 118.5, 122.8, 124.5, 
127.6, 129.3, 129.7, 130.1, 130.5, 131.3, 131.4, 137.7, 140.4, 140.8, 147.4; HRMS (FAB): $m / z$ calcd for $\mathrm{C}_{22} \mathrm{H}_{23} \mathrm{~N}_{4} \mathrm{~S}[\mathrm{M}+\mathrm{H}]^{+}$375.1643; found: 375.1646 .

Compound $\mathbf{3 1 j}$. Using the general procedure as described for $\mathbf{2 5 a}$, compound 29j (40.3 mg, $0.11 \mathrm{mmol}$ ) was allowed to react for $2 \mathrm{~h}$. Purification by flash chromatography over aluminum oxide with $n$-hexane-EtOAc $(2: 1$ to $0: 1)$ gave the title compound $\mathbf{3 1 j}$ as colorless solid (30.6 mg, $89 \%$ ): $\mathrm{mp} 196-197{ }^{\circ} \mathrm{C}$ (from $\mathrm{MeOH}-\mathrm{CHCl}_{3}-n$-hexane); IR (neat) $\mathrm{cm}^{-1}: 2230(\mathrm{C} \equiv \mathrm{N}), 1623(\mathrm{C}=\mathrm{N}), 1572$ $(\mathrm{C}=\mathrm{N}) ;{ }^{1} \mathrm{H}-\mathrm{NMR}\left(400 \mathrm{MHz}, \mathrm{CDCl}_{3}-\mathrm{CD}_{3} \mathrm{OD}\right) \delta: 1.98-2.04\left(\mathrm{~m}, 2 \mathrm{H}, \mathrm{CH}_{2}\right), 3.71(\mathrm{t}$, $\left.J=5.5 \mathrm{~Hz}, 2 \mathrm{H}, \mathrm{CH}_{2}\right), 4.01\left(\mathrm{t}, J=6.1 \mathrm{~Hz}, 2 \mathrm{H}, \mathrm{CH}_{2}\right), 7.25(\mathrm{~d}, J=1.8 \mathrm{~Hz}, 1 \mathrm{H}$, Ar), $7.44(\mathrm{dd}, J=8.5,1.8 \mathrm{~Hz}, 1 \mathrm{H}, \mathrm{Ar}), 7.58(\mathrm{t}, J=7.8 \mathrm{~Hz}, 1 \mathrm{H}, \mathrm{Ar}), 7.68$ (d, $J=7.8 \mathrm{~Hz}, 1 \mathrm{H}, \mathrm{Ar}), 7.81(\mathrm{~d}, J=7.8 \mathrm{~Hz}, 1 \mathrm{H}, \mathrm{Ar}), 7.86(\mathrm{~s}, 1 \mathrm{H}, \mathrm{Ar}), 8.27$ (d, $J=8.5 \mathrm{~Hz}, 1 \mathrm{H}, \mathrm{Ar}) ;{ }^{13} \mathrm{C}-\mathrm{NMR}\left(100 \mathrm{MHz}, \mathrm{CDCl}_{3}-\mathrm{CD}_{3} \mathrm{OD}\right) \delta: 20.7,43.9,44.7$, 113.0, 118.3, 121.9, 124.9, 126.2, 129.5, 129.7, 129.7, 130.4, 131.3, 131.5, 140.2, 141.0, 146.6, 153.4; HRMS (FAB): $m / z$ calcd for $\mathrm{C}_{18} \mathrm{H}_{15} \mathrm{~N}_{4} \mathrm{~S}[\mathrm{M}+\mathrm{H}]^{+} 319.1017$; found: 319.1016 .

\subsubsection{Synthesis of 3,4-Dihydro-9-(3-nitrophenyl)-2H,6H- pyrimido[1,2-c][1,3]benzothiazin-6-imine $(31 \mathrm{k})$}

\section{$N$-(tert-Butyl)-3,4-dihydro-9-(3-nitrophenyl)-2H,6H-pyrimido[1,2-c][1,3]ben-} zothiazin-6-imine $(\mathbf{2 9 k})$. Using the general procedure as described for $\mathbf{2 2}$, $\mathrm{N}$-(tert-butyl)-9-bromo-3,4-dihydro-2 H,6H-pyrimido[1,2-c][1,3]benzothiazin-6imine $22 \mathbf{k}(52.8 \mathrm{mg}, 0.15 \mathrm{mmol})$ was allowed to react with 3-nitrophenylboronic acid $(30.0 \mathrm{mg}, 0.18 \mathrm{mmol}$ ) for $1 \mathrm{~h}$. Purification by flash chromatography over aluminum oxide with $n$-hexane-EtOAc (1:0 to 8:2) gave the title compound 29k as colorless solid (52.3 mg, $88 \%$ ): mp 208-209 ${ }^{\circ} \mathrm{C}$ (from $\mathrm{CHCl}_{3}-n$-hexane); IR (neat) $\mathrm{cm}^{-1}: 1593(\mathrm{C}=\mathrm{N}), 1529\left(\mathrm{NO}_{2}\right) ;{ }^{1} \mathrm{H}-\mathrm{NMR}\left(500 \mathrm{MHz}, \mathrm{CDCl}_{3}\right) \delta: 1.41(\mathrm{~s}$, $\left.9 \mathrm{H}, 3 \times \mathrm{CH}_{3}\right), 1.92-1.96\left(\mathrm{~m}, 2 \mathrm{H}, \mathrm{CH}_{2}\right), 3.65\left(\mathrm{t}, J=5.4 \mathrm{~Hz}, 2 \mathrm{H}, \mathrm{CH}_{2}\right), 3.90(\mathrm{t}$, $\left.J=6.3 \mathrm{~Hz}, 2 \mathrm{H}, \mathrm{CH}_{2}\right), 7.37(\mathrm{~d}, J=1.7 \mathrm{~Hz}, 1 \mathrm{H}, \mathrm{Ar}), 7.44(\mathrm{dd}, J=8.6,1.7 \mathrm{~Hz}$, $1 \mathrm{H}, \mathrm{Ar}), 7.62(\mathrm{t}, J=8.0 \mathrm{~Hz}, 1 \mathrm{H}, \mathrm{Ar}), 7.91(\mathrm{dd}, J=8.0,2.0 \mathrm{~Hz}, 1 \mathrm{H}, \mathrm{Ar}), 8.22$ (dd, $J=8.0,2.0 \mathrm{~Hz}, 1 \mathrm{H}, \mathrm{Ar}), 8.31(\mathrm{~d}, J=8.6 \mathrm{~Hz}, 1 \mathrm{H}, \mathrm{Ar}), 8.44(\mathrm{t}, J=2.0 \mathrm{~Hz}, 1 \mathrm{H}$, $\mathrm{Ar}) ;{ }^{13} \mathrm{C}-\mathrm{NMR}\left(125 \mathrm{MHz}, \mathrm{CDCl}_{3}\right) \delta: 21.8,30.0$ (3C), 45.2, 45.4, 54.3, 121.8, $122.7,122.9,124.6,127.7,129.4,129.9,130.2,132.9,137.6,140.2,141.1,147.4$, 148.7; HRMS (FAB): $m / z$ calcd for $\mathrm{C}_{21} \mathrm{H}_{23} \mathrm{~N}_{4} \mathrm{O}_{2} \mathrm{~S}[\mathrm{M}+\mathrm{H}]^{+} 395.1542$; found: 395.1544 .

Compound 31k. Using the general procedure as described for 25a, compound 29k (37.9 mg, $0.096 \mathrm{mmol}$ ) was allowed to react for $3 \mathrm{~h}$. Purification by flash chromatography over aluminum oxide with $n$-hexane-EtOAc $(1: 1)$ gave the title compound 31k as colorless solid $\left(23.2 \mathrm{mg}, 71 \%\right.$ ): $\mathrm{mp} 168-170{ }^{\circ} \mathrm{C}$ (from $\mathrm{CHCl}_{3}-$ $n$-hexane); IR (neat) $\mathrm{cm}^{-1}$ : $1620(\mathrm{C}=\mathrm{N}), 1567(\mathrm{C}=\mathrm{N}), 1529\left(\mathrm{NO}_{2}\right) ;{ }^{1} \mathrm{H}-\mathrm{NMR}$ $\left(500 \mathrm{MHz}, \mathrm{CDCl}_{3}\right) \delta: 1.98-2.03\left(\mathrm{~m}, 2 \mathrm{H}, \mathrm{CH}_{2}\right), 3.72\left(\mathrm{t}, J=5.7 \mathrm{~Hz}, 2 \mathrm{H}, \mathrm{CH}_{2}\right), 4.05$ $\left(\mathrm{t}, J=6.0 \mathrm{~Hz}, 2 \mathrm{H}, \mathrm{CH}_{2}\right), 7.28(\mathrm{~d}, J=1.7 \mathrm{~Hz}, 1 \mathrm{H}, \mathrm{Ar}), 7.47(\mathrm{dd}, J=8.6,1.7 \mathrm{~Hz}$, 
1H, Ar), $7.63(\mathrm{t}, J=7.7 \mathrm{~Hz}, 1 \mathrm{H}, \mathrm{Ar}), 7.90(\mathrm{dd}, J=7.7,1.7 \mathrm{~Hz}, 1 \mathrm{H}, \mathrm{Ar}), 8.23$ (dd, $J=7.7,1.7 \mathrm{~Hz}, 1 \mathrm{H}, \mathrm{Ar}), 8.34(\mathrm{~d}, J=8.6 \mathrm{~Hz}, 1 \mathrm{H}, \mathrm{Ar}), 8.42(\mathrm{t}, J=1.7 \mathrm{~Hz}, 1 \mathrm{H}$, $\mathrm{Ar}) ;{ }^{13} \mathrm{C}-\mathrm{NMR}\left(125 \mathrm{MHz}, \mathrm{CDCl}_{3}\right) \delta: 21.0,43.8,45.0,121.8,121.9,122.9,124.9$, 126.7, 129.8, 129.9, 130.0, 132.8, 140.7, 140.8, 146.0, 148.7, 152.8; Anal. calcd for $\mathrm{C}_{17} \mathrm{H}_{14} \mathrm{~N}_{4} \mathrm{O}_{2} \mathrm{~S}$ : C, 60.34; H, 4.17; N, 16.56. Found: C, 60.04; H, 4.13; N, 16.28.

\title{
3.1.49 Synthesis of ( \pm )-3,4-Dihydro-9-[3-(1- hydroxyethyl)phenyl]-2H,6H-pyrimido [1,2-c][1,3]benzothiazin-6-imine (31l)
}

\begin{abstract}
$N$-(tert-Butyl)-3,4-dihydro-9-(3-vinylphenyl)-2H,6H-pyrimido[1,2-c][1,3]benzothiazin-6-imine (29l). Using the general procedure as described for $22 \mathrm{l}, \mathrm{N}$-(tertbutyl)-9-bromo-3,4-dihydro-2H,6H-pyrimido[1,2-c][1,3]benzothiazin-6-imine 22k (70.6 mg, $0.20 \mathrm{mmol}$ ) was allowed to react with 3-vinylphenylboronic acid (35.5 mg, $0.24 \mathrm{mmol}$ ) for $1 \mathrm{~h}$. Purification by flash chromatography over aluminum oxide with $n$-hexane-EtOAc (1:0 to 9:1) gave the title compound 291 as a colorless oil (64.4 mg, $86 \%$ ): IR (neat) $\mathrm{cm}^{-1}$ : $1591(\mathrm{C}=\mathrm{N}) ;{ }^{1} \mathrm{H}-\mathrm{NMR}\left(500 \mathrm{MHz}, \mathrm{CDCl}_{3}\right) \delta$ : $1.40\left(\mathrm{~s}, 9 \mathrm{H}, 3 \times \mathrm{CH}_{3}\right), 1.90-1.95\left(\mathrm{~m}, 2 \mathrm{H}, \mathrm{CH}_{2}\right), 3.64\left(\mathrm{t}, J=5.4 \mathrm{~Hz}, 2 \mathrm{H}, \mathrm{CH}_{2}\right), 3.89$ $\left(\mathrm{t}, J=6.3 \mathrm{~Hz}, 2 \mathrm{H}, \mathrm{CH}_{2}\right), 5.30(\mathrm{~d}, J=10.9 \mathrm{~Hz}, 1 \mathrm{H}, \mathrm{CH}), 5.82(\mathrm{~d}, J=17.8 \mathrm{~Hz}, 1 \mathrm{H}$, $\mathrm{CH}), 6.77$ (dd, $J=17.8,10.9 \mathrm{~Hz}, 1 \mathrm{H}, \mathrm{CH}), 7.34$ (d, $J=1.7 \mathrm{~Hz}, 1 \mathrm{H}, \mathrm{Ar}), 7.37-7.47$ (m, 4H, Ar), 7.59 (s, 1H, Ar), 8.25 (d, $J=8.6 \mathrm{~Hz}, 1 \mathrm{H}, \mathrm{Ar}) ;{ }^{13} \mathrm{C}-\mathrm{NMR}(125 \mathrm{MHz}$, $\left.\mathrm{CDCl}_{3}\right) \delta: 21.9,30.0$ (3C), 45.1, 45.4, 54.2, 114.5, 122.7, 124.8, 125.0, 125.8, 126.5, 126.6, 128.9, 129.0, 129.5, 136.5, 138.2, 138.2, 139.7, 142.8, 147.7; HRMS (FAB): $m / z$ calcd for $\mathrm{C}_{23} \mathrm{H}_{26} \mathrm{~N}_{3} \mathrm{~S}[\mathrm{M}+\mathrm{H}]^{+} 376.1847$; found: 376.1850 .
\end{abstract}

Compound 311. Using the general procedure as described for $\mathbf{2 5 a}$, compound 291 (58.5 mg, $0.16 \mathrm{mmol}$ ) was allowed to react for $3 \mathrm{~h}$. Purification by flash chromatography over aluminum oxide with $n$-hexane-EtOAc $(9: 1$ to $1: 1)$ gave the title compound $\mathbf{3 1 1}$ as colorless solid (25.9 mg, $49 \%$ ): $\mathrm{mp} 193-195{ }^{\circ} \mathrm{C}$ (from $\mathrm{CHCl}_{3}-n$-hexane); IR (neat) $\mathrm{cm}^{-1}: 1616(\mathrm{C}=\mathrm{N}), 1558 \quad(\mathrm{C}=\mathrm{N}) ;{ }^{1} \mathrm{H}-\mathrm{NMR}$ $\left(400 \mathrm{MHz}, \mathrm{DMSO}-d_{6}\right) \delta: 1.36\left(\mathrm{~d}, J=6.6 \mathrm{~Hz}, 3 \mathrm{H}, \mathrm{CH}_{3}\right), 1.84-1.89\left(\mathrm{~m}, 2 \mathrm{H}, \mathrm{CH}_{2}\right)$, $3.59\left(\mathrm{t}, J=5.1 \mathrm{~Hz}, 2 \mathrm{H}, \mathrm{CH}_{2}\right), 3.92\left(\mathrm{t}, J=6.0 \mathrm{~Hz}, 2 \mathrm{H}, \mathrm{CH}_{2}\right), 4.76-4.81(\mathrm{~m}, 1 \mathrm{H}$, $\mathrm{CH}), 5.20(\mathrm{~d}, J=4.4 \mathrm{~Hz}, 1 \mathrm{H}, \mathrm{OH}), 7.36-7.43$ (m, 2H, Ar), 7.53-7.57 (m, 3H, Ar), $7.67(\mathrm{~s}, 1 \mathrm{H}, \mathrm{Ar}), 8.24(\mathrm{~d}, J=8.3 \mathrm{~Hz}, 1 \mathrm{H}, \mathrm{Ar}), 8.71(\mathrm{~s}, 1 \mathrm{H}, \mathrm{NH}) ;{ }^{13} \mathrm{C}-\mathrm{NMR}$ (125 MHz, $\left.\mathrm{CDCl}_{3}-\mathrm{CD}_{3} \mathrm{OD}\right) \delta:$ 20.6, 25.0, 44.0, 44.5, 69.5, 121.7, 123.8, 125.0, 125.1, 125.3, 125.6, 128.8, 129.0, 129.0, 138.8, 143.6, 146.8, 147.2, 154.1; HRMS (FAB): $m / z$ calcd for $\mathrm{C}_{19} \mathrm{H}_{20} \mathrm{~N}_{3} \mathrm{OS}[\mathrm{M}+\mathrm{H}]^{+} 338.1327$; found: 338.1327 . 


\subsubsection{Synthesis of 9-[3-(Acetylamino)phenyl]-3,4-dihydro- 2H,6H-pyrimido[1,2-c][1,3]benzothiazin-6-imine (31m)}

9-[3-(Acetylamino)phenyl]- $\mathrm{N}$-(tert-butyl)-3,4-dihydro-2H,6H-pyrimido[1,2-c] [1,3]benzothiazin-6-imine $\mathbf{( 2 9 m )}$. Using the general procedure as described for 22l, $\mathrm{N}$-(tert-butyl)-9-bromo-3,4-dihydro-2H,6H-pyrimido[1,2-c][1,3]benzothiazin6-imine $22 \mathbf{k}(52.8 \mathrm{mg}, 0.15 \mathrm{mmol})$ was allowed to react with 3-(acetylamino)phenylboronic acid (32.2 $\mathrm{mg}, 0.18 \mathrm{mmol}$ ) in 1,4-dioxane for $1 \mathrm{~h}$. Purification by flash chromatography over aluminum oxide with $n$-hexane-EtOAc (1:1) gave the title compound 29m as colorless solid (44.6 mg, $73 \%$ ): $\mathrm{mp} 221-222{ }^{\circ} \mathrm{C}$ (from $\mathrm{CHCl}_{3}-n$-hexane); IR (neat) $\mathrm{cm}^{-1}: 1670(\mathrm{C}=\mathrm{O}), 1590(\mathrm{C}=\mathrm{N}) ;{ }^{1} \mathrm{H}-\mathrm{NMR}(500 \mathrm{MHz}$, $\left.\mathrm{CDCl}_{3}\right) \delta: 1.40\left(\mathrm{~s}, 9 \mathrm{H}, 3 \times \mathrm{CH}_{3}\right), 1.91-1.95\left(\mathrm{~m}, 2 \mathrm{H}, \mathrm{CH}_{2}\right), 2.18\left(\mathrm{~s}, 3 \mathrm{H}, \mathrm{CH}_{3}\right), 3.64(\mathrm{t}$, $\left.J=5.4 \mathrm{~Hz}, 2 \mathrm{H}, \mathrm{CH}_{2}\right), 3.89\left(\mathrm{t}, J=6.0 \mathrm{~Hz}, 2 \mathrm{H}, \mathrm{CH}_{2}\right), 7.29-7.38(\mathrm{~m}, 4 \mathrm{H}, \mathrm{Ar}), 7.50$ $(\mathrm{d}, J=7.4 \mathrm{~Hz}, 1 \mathrm{H}, \mathrm{Ar}), 7.70(\mathrm{~s}, 1 \mathrm{H}, \mathrm{NH}), 7.73(\mathrm{~s}, 1 \mathrm{H}, \mathrm{Ar}), 8.21(\mathrm{~d}, J=8.0 \mathrm{~Hz}, 1 \mathrm{H}$, $\mathrm{Ar}) ;{ }^{13} \mathrm{C}-\mathrm{NMR}\left(125 \mathrm{MHz}, \mathrm{CDCl}_{3}\right) \delta: 21.9,24.5,30.0$ (3C), 45.1, 45.4, 54.2, 118.4, $119.5,122.8,122.9,124.8,126.7,128.8,129.5,129.5,138.2,138.5,140.2,142.4$, 147.9, 168.5; HRMS (FAB): $m / z$ calcd for $\mathrm{C}_{23} \mathrm{H}_{27} \mathrm{~N}_{4} \mathrm{OS}[\mathrm{M}+\mathrm{H}]^{+} 407.1906$; found: 407.1905.

Compound $\mathbf{3 1 \mathrm { m }}$. Using the general procedure as described for $\mathbf{2 5 \mathbf { a }}$, compound 29m (35.4 mg, $0.096 \mathrm{mmol}$ ) was allowed to react for $3 \mathrm{~h}$. Purification by preparative TLC over aluminum oxide with EtOAc-MeOH (98:2) gave the title compound 31m as colorless solid $\left(23.7 \mathrm{mg}, 78 \%\right.$ ): $\mathrm{mp} 208-210{ }^{\circ} \mathrm{C}$ (from $\mathrm{MeOH}-$ $\mathrm{CHCl}_{3}-n$-hexane); IR (neat) $\mathrm{cm}^{-1}: 1691(\mathrm{C}=\mathrm{O}), 1611(\mathrm{C}=\mathrm{N}), 1561(\mathrm{C}=\mathrm{N}) ;{ }^{1} \mathrm{H}-$ NMR (DMSO- $\left.d_{6}\right) \delta: 1.85-1.90\left(\mathrm{~m}, 2 \mathrm{H}, \mathrm{CH}_{2}\right), 2.06\left(\mathrm{~s}, 3 \mathrm{H}, \mathrm{CH}_{3}\right), 3.60$ (t, $\left.J=5.4 \mathrm{~Hz}, 2 \mathrm{H}, \mathrm{CH}_{2}\right), 3.93\left(\mathrm{t}, J=6.0 \mathrm{~Hz}, 2 \mathrm{H}, \mathrm{CH}_{2}\right), 7.37-7.41(\mathrm{~m}, 2 \mathrm{H}, \mathrm{Ar})$, 7.46-7.49 (m, 2H, Ar), 7.61 (d, $J=6.3 \mathrm{~Hz}, 1 \mathrm{H}, \mathrm{Ar}), 7.90$ (s, 1H, Ar), 8.26 (d, $J=8.0 \mathrm{~Hz}, 1 \mathrm{H}, \mathrm{Ar}), 8.75(\mathrm{~s}, 1 \mathrm{H}, \mathrm{NH}), 10.04(\mathrm{~s}, 1 \mathrm{H}, \mathrm{NH}) ;{ }^{13} \mathrm{C}-\mathrm{NMR}(125 \mathrm{MHz}$, $\left.\mathrm{CDCl}_{3}-\mathrm{CD}_{3} \mathrm{OD}\right) \delta: 20.7,23.7,44.0,44.5,118.3,119.6,121.8,122.4,125.1$ (2C), 129.0, 129.0, 129.2, 138.8, 139.4, 143.3, 147.2, 154.1, 169.7; HRMS (FAB): $m / z$ calcd for $\mathrm{C}_{19} \mathrm{H}_{17} \mathrm{~N}_{4} \mathrm{OS}[\mathrm{M}-\mathrm{H}]^{-}$349.1123; found: 349.1129 .

\subsubsection{Synthesis of 3,4-Dihydro-9-[3- (methanesulfonylamino)phenyl]-2H,6H- pyrimido[1,2-c][1,3]benzothiazin-6-imine (31n)}

$\mathrm{N}$-(tert-Butyl)-3,4-dihydro-9-[3-(methanesulfonylamino)phenyl]-2H,6H-pyrimido[1,2-c][1,3]benzothiazin-6-imine (29n). Using the general procedure as described for 22l, $N$-(tert-butyl)-9-bromo-3,4-dihydro-2H,6H-pyrimido[1,2-c] [1,3] benzothiazin-6-imine $22 \mathbf{k}(52.8 \mathrm{mg}, 0.15 \mathrm{mmol})$ was allowed to react with 3-(methanesulfonylamino)phenylboronic acid (38.7 mg, $0.18 \mathrm{mmol}$ ) in 1,4-dioxane 
for $1 \mathrm{~h}$. Purification by flash chromatography over aluminum oxide with $n$-hexaneEtOAc (1:2 to 0:1) gave the title compound 29n as colorless solid (20.1 mg, $30 \%)$ : mp 200-202 ${ }^{\circ} \mathrm{C}$ (from $\mathrm{CHCl}_{3}-n$-hexane); IR (neat) $\mathrm{cm}^{-1}$ : $1591(\mathrm{C}=\mathrm{N}), 1153$ $\left(\mathrm{NSO}_{2}\right) ;{ }^{1} \mathrm{H}-\mathrm{NMR}\left(500 \mathrm{MHz}, \mathrm{CDCl}_{3}-\mathrm{CD}_{3} \mathrm{OD}\right) \delta: 1.41\left(\mathrm{~s}, 9 \mathrm{H}, 3 \times \mathrm{CH}_{3}\right), 1.92-1.97$ $\left(\mathrm{m}, 2 \mathrm{H}, \mathrm{CH}_{2}\right), 3.02\left(\mathrm{~s}, 3 \mathrm{H}, \mathrm{CH}_{3}\right), 3.62\left(\mathrm{t}, J=5.2 \mathrm{~Hz}, 2 \mathrm{H}, \mathrm{CH}_{2}\right), 3.90(\mathrm{t}, J=6.0 \mathrm{~Hz}$, $\left.2 \mathrm{H}, \mathrm{CH}_{2}\right), 7.27(\mathrm{~d}, J=7.4 \mathrm{~Hz}, 1 \mathrm{H}, \mathrm{Ar}), 7.34-7.43(\mathrm{~m}, 5 \mathrm{H}, \mathrm{Ar}), 8.15(\mathrm{~d}, J=8.6 \mathrm{~Hz}$, $1 \mathrm{H}, \mathrm{Ar}) ;{ }^{13} \mathrm{C}-\mathrm{NMR}\left(125 \mathrm{MHz}, \mathrm{CDCl}_{3}\right) \delta: 21.8,30.0$ (3C), 39.5, 44.9, 45.5, 54.3, 119.1, 119.8, 122.9, 123.7, 124.8, 127.0, 129.0, 129.8, 130.1, 137.7, 137.9, 140.9, 142.0, 148.4; HRMS (FAB): $m / z$ calcd for $\mathrm{C}_{22} \mathrm{H}_{27} \mathrm{~N}_{4} \mathrm{O}_{2} \mathrm{~S}_{2}[\mathrm{M}+\mathrm{H}]^{+} 443.1575$; found: 443.1574 .

Compound 31n. Using the general procedure as described for 25a, compound 29n $(26.1 \mathrm{mg}, 0.059 \mathrm{mmol})$ was allowed to react for $4.5 \mathrm{~h}$. Purification by preparative TLC over aluminum oxide with EtOAc-MeOH (95:5) gave the title compound 31n as colorless solid (13.4 mg, $59 \%$ ): $\mathrm{mp} 194-196{ }^{\circ} \mathrm{C}$ (from MeOH$\mathrm{CHCl}_{3}-n$-hexane); IR (neat) $\mathrm{cm}^{-1}: 1625(\mathrm{C}=\mathrm{N}), 1557(\mathrm{C}=\mathrm{N}), 1325\left(\mathrm{NSO}_{2}\right), 1147$ $\left(\mathrm{NSO}_{2}\right) ;{ }^{1} \mathrm{H}-\mathrm{NMR}\left(500 \mathrm{MHz}, \mathrm{CDCl}_{3}-\mathrm{CD}_{3} \mathrm{OD}\right) \delta: 1.98-2.03\left(\mathrm{~m}, 2 \mathrm{H}, \mathrm{CH}_{2}\right), 3.02(\mathrm{~s}$, $\left.3 \mathrm{H}, \mathrm{CH}_{3}\right), 3.70\left(\mathrm{t}, J=5.4 \mathrm{~Hz}, 2 \mathrm{H}, \mathrm{CH}_{2}\right), 4.00\left(\mathrm{t}, J=6.3 \mathrm{~Hz}, 2 \mathrm{H}, \mathrm{CH}_{2}\right)$, 7.23-7.46 (m, 6H, Ar), 8.21 (d, $J=8.6 \mathrm{~Hz}, 1 \mathrm{H}, \mathrm{Ar}) ;{ }^{13} \mathrm{C}-\mathrm{NMR}(125 \mathrm{MHz}$, $\left.\mathrm{CDCl}_{3}-\mathrm{CD}_{3} \mathrm{OD}\right) \delta: 20.8,39.1,44.0,44.7,118.8,120.0,121.9,123.4,125.2,125.6$, 129.3 , 129.5, 130.1 138.0, 140.5, 142.7, 147.0, 153.9; HRMS (FAB): $m / z$ calcd for $\mathrm{C}_{18} \mathrm{H}_{19} \mathrm{~N}_{4} \mathrm{O}_{2} \mathrm{~S}_{2}[\mathrm{M}+\mathrm{H}]^{+}$387.0949; found: 387.0957 .

\subsubsection{Synthesis of 3,4-Dihydro-9-(3-hydroxyphenyl)- 2H,6H-pyrimido[1,2-c][1,3]benzothiazin-6-imine (31o)}

$N$-(tert-Butyl)-3,4-dihydro-9-(3-hydroxyphenyl)-2H,6H-pyrimido[1,2-c][1,3] benzothiazin-6-imine (290). Using the general procedure as described for 221, $\mathrm{N}$-(tert-butyl)-9-bromo-3,4-dihydro-2H,6H-pyrimido[1,2-c][1,3]benzothiazin-6-imine 22k (52.8 $\mathrm{mg}, 0.15 \mathrm{mmol}$ ) was allowed to react with 3-hydroxyphenylboronic acid $(24.8 \mathrm{mg}, 0.18 \mathrm{mmol})$ in 1,4-dioxane for $1 \mathrm{~h}$. Purification by flash chromatography over aluminum oxide with $n$-hexane-EtOAc (1:1 to $0: 1)$ gave the title compound 290 as colorless solid (16.1 mg, $29 \%$ ): mp 265-267 ${ }^{\circ} \mathrm{C}$ (from MeOH$\mathrm{CHCl}_{3}-n$-hexane); IR (neat) $\mathrm{cm}^{-1}: 1591(\mathrm{C}=\mathrm{N}) ;{ }^{1} \mathrm{H}-\mathrm{NMR}\left(500 \mathrm{MHz}, \mathrm{CDCl}_{3}-\right.$ $\left.\mathrm{CD}_{3} \mathrm{OD}\right) \delta: 1.41\left(\mathrm{~s}, 9 \mathrm{H}, 3 \times \mathrm{CH}_{3}\right), 1.92-1.97\left(\mathrm{~m}, 2 \mathrm{H}, \mathrm{CH}_{2}\right), 3.60(\mathrm{t}, J=5.2 \mathrm{~Hz}$, $\left.2 \mathrm{H}, \mathrm{CH}_{2}\right), 3.90\left(\mathrm{t}, J=6.0 \mathrm{~Hz}, 2 \mathrm{H}, \mathrm{CH}_{2}\right), 6.85$ (d, $\left.J=8.0 \mathrm{~Hz}, 1 \mathrm{H}, \mathrm{Ar}\right), 7.03$ (s, $1 \mathrm{H}, \mathrm{Ar}), 7.08$ (d, $J=8.0 \mathrm{~Hz}, 1 \mathrm{H}, \mathrm{Ar}), 7.27$ (t, $J=8.0 \mathrm{~Hz}, 1 \mathrm{H}, \mathrm{Ar}), 7.34$ (s, 1H, Ar), $7.43(\mathrm{~d}, J=8.0 \mathrm{~Hz}, 1 \mathrm{H}, \mathrm{Ar}), 8.09$ (d, $J=8.0 \mathrm{~Hz}, 1 \mathrm{H}, \mathrm{Ar}) ;{ }^{13} \mathrm{C}-\mathrm{NMR}$ $\left(125 \mathrm{MHz}, \mathrm{CDCl}_{3}-\mathrm{CD}_{3} \mathrm{OD}\right) \delta: 21.5,29.7$ (3C), 44.5, 45.4, 54.2, 113.8, 115.1, 118.2, 122.8, 124.9, 126.0, 128.5, 129.4, 129.8, 138.1, 140.4, 143.2, 149.4, 157.2; HRMS (FAB): $m / z$ calcd for $\mathrm{C}_{21} \mathrm{H}_{24} \mathrm{~N}_{3} \mathrm{OS}[\mathrm{M}+\mathrm{H}]^{+}$366.1640; found: 366.1639. 
Compound 310. TFA (9 mL) was added to a mixture of 29o (16.1 mg, $0.044 \mathrm{mmol})$ and MS4 $\mathrm{A}$ (2.0 g, powder, activated by heating with Bunsen burner) in $\mathrm{CHCl}_{3}(1.0 \mathrm{~mL})$ and $\mathrm{MeOH}$ (10 drops). After being stirred under reflux for $5 \mathrm{~h}$, the mixture was concentrated. To a mixture of the residue in $\mathrm{CHCl}_{3}$ was added dropwise $\mathrm{Et}_{3} \mathrm{~N}$ at $0{ }^{\circ} \mathrm{C}$ to adjust $\mathrm{pH}$ to $8-9$. The whole was extracted with EtOAc. The extract was washed with sat. $\mathrm{NaHCO}_{3}$, brine, and dried over $\mathrm{MgSO}_{4}$. After concentration, the residue was purified by preparative TLC over aluminum oxide with EtOAc-MeOH (95:5) to give the title compound 310 as colorless solid (7.9 mg, $58 \%$ ): $\mathrm{mp} 199-200{ }^{\circ} \mathrm{C}$ (from $\mathrm{MeOH}-\mathrm{CHCl}_{3}-n$-hexane); IR (neat) $\mathrm{cm}^{-1}$ : $1621(\mathrm{C}=\mathrm{N}), 1557(\mathrm{C}=\mathrm{N}) ;{ }^{1} \mathrm{H}-\mathrm{NMR}\left(500 \mathrm{MHz}, \mathrm{DMSO}-d_{6}\right) \delta: 1.85-1.90(\mathrm{~m}, 2 \mathrm{H}$, $\left.\mathrm{CH}_{2}\right), 3.60\left(\mathrm{t}, J=5.4 \mathrm{~Hz}, 2 \mathrm{H}, \mathrm{CH}_{2}\right), 3.92\left(\mathrm{t}, J=5.4 \mathrm{~Hz}, 2 \mathrm{H}, \mathrm{CH}_{2}\right), 6.81(\mathrm{dd}$, $J=7.7,2.0 \mathrm{~Hz}, 1 \mathrm{H}, \mathrm{Ar}), 7.06(\mathrm{t}, J=2.0 \mathrm{~Hz}, 1 \mathrm{H}, \mathrm{Ar}), 7.13(\mathrm{~d}, J=8.3 \mathrm{~Hz}, 1 \mathrm{H}$, Ar), 7.27 (t, $J=7.7 \mathrm{~Hz}, 1 \mathrm{H}, \mathrm{Ar}), 7.48-7.50(\mathrm{~m}, 2 \mathrm{H}, \mathrm{Ar}), 8.22(\mathrm{~d}, J=8.3 \mathrm{~Hz}, 1 \mathrm{H}$, Ar), 8.74 (br s, 1H, NH), 9.57 (s, 1H, OH); ${ }^{13} \mathrm{C}-\mathrm{NMR}\left(100 \mathrm{MHz}, \mathrm{DMSO}-d_{6}\right) \delta$ : 20.7, 43.1, 44.3, 113.5, 115.3, 117.5, 121.4, 124.2, 124.9, 129.0, 129.5, 130.0, 139.5, 142.3, 145.2, 149.7, 157.8; HRMS (FAB): $\mathrm{m} / z$ calcd for $\mathrm{C}_{17} \mathrm{H}_{16} \mathrm{~N}_{3} \mathrm{OS}$ $[\mathrm{M}+\mathrm{H}]^{+}$310.1014; found: 310.1010 .

\subsubsection{Synthesis of 3,4-Dihydro-9-(4-methoxyphenyl)- 2H,6H-pyrimido[1,2-c][1,3]benzothiazin-6-imine (31p)}

$N$-(tert-Butyl)-3,4-dihydro-9-(3-methoxyphenyl)-2H,6H-pyrimido[1,2-c][1,3] benzothiazin-6-imine (29p). Using the general procedure as described for 22l, $\mathrm{N}$ (tert-butyl)-9-bromo-3,4-dihydro-2H,6H-pyrimido[1,2-c][1,3]benzothiazin-6-imine 22k (52.8 $\mathrm{mg}, 0.15 \mathrm{mmol})$ was allowed to react with 4-methoxyphenylboronic acid (27.4 mg, $0.18 \mathrm{mmol}$ ) for $1 \mathrm{~h}$. Purification by flash chromatography over aluminum oxide with $n$-hexane-EtOAc (1:0 to 9:1) gave the title compound 29p as a colorless oil (55.6 mg, $98 \%$ ): IR (neat) $\mathrm{cm}^{-1}: 1591(\mathrm{C}=\mathrm{N}) ;{ }^{1} \mathrm{H}-\mathrm{NMR}\left(400 \mathrm{MHz}, \mathrm{CDCl}_{3}\right) \delta$ : $1.40\left(\mathrm{~s}, 9 \mathrm{H}, 3 \times \mathrm{CH}_{3}\right), 1.90-1.96\left(\mathrm{~m}, 2 \mathrm{H}, \mathrm{CH}_{2}\right), 3.64\left(\mathrm{t}, J=5.5 \mathrm{~Hz}, 2 \mathrm{H}, \mathrm{CH}_{2}\right), 3.86$ (s, 3H, $\left.\mathrm{CH}_{3}\right), 3.89\left(\mathrm{t}, J=6.2 \mathrm{~Hz}, 2 \mathrm{H}, \mathrm{CH}_{2}\right), 6.91(\mathrm{dd}, J=8.2,2.5 \mathrm{~Hz}, 1 \mathrm{H}, \mathrm{Ar})$, $7.10(\mathrm{t}, J=2.5 \mathrm{~Hz}, 1 \mathrm{H}, \mathrm{Ar}), 7.15-7.18$ (m, 1H, Ar), 7.32-7.37 (m, 2H, Ar), 7.41 $(\mathrm{dd}, J=8.5,1.7 \mathrm{~Hz}, 1 \mathrm{H}, \mathrm{Ar}), 8.24(\mathrm{~d}, J=8.5 \mathrm{~Hz}, 1 \mathrm{H}, \mathrm{Ar}) ;{ }^{13} \mathrm{C}-\mathrm{NMR}(100 \mathrm{MHz}$, $\mathrm{CDCl}_{3}$ ) $\delta: 21.9,30.0$ (3C), 45.1, 45.4, 54.2, 55.3, 112.7, 113.5, 119.5, 122.8, 124.9, 126.7, 128.9, 129.5, 129.9, 138.2, 140.9, 142.8, 147.7, 160.0; HRMS (FAB): $\mathrm{m} / \mathrm{z}$ calcd for $\mathrm{C}_{22} \mathrm{H}_{26} \mathrm{~N}_{3} \mathrm{OS}[\mathrm{M}+\mathrm{H}]^{+}$380.1797; found: 380.1793 .

Compound 31p. Using the general procedure as described for 25a, compound 29p (32.1 mg, $0.085 \mathrm{mmol})$ was allowed to react for $2 \mathrm{~h}$ with TFA $(1.0 \mathrm{~mL})$ and MS4Å (150 mg). Purification by flash chromatography over aluminum oxide with $n$-hexane-EtOAc (3:1) gave the title compound 31p as colorless solid (23.4 $\mathrm{mg}$, $85 \%$ ): $\mathrm{mp} 114-115^{\circ} \mathrm{C}$ (from $\mathrm{CHCl}_{3}-n$-hexane); IR (neat) $\mathrm{cm}^{-1}$ : $1620(\mathrm{C}=\mathrm{N}$ ), $1569(\mathrm{C}=\mathrm{N}) ;{ }^{1} \mathrm{H}-\mathrm{NMR}\left(400 \mathrm{MHz}, \mathrm{CDCl}_{3}\right) \delta: 1.96-2.02\left(\mathrm{~m}, 2 \mathrm{H}, \mathrm{CH}_{2}\right), 3.71(\mathrm{t}$, 
$\left.J=5.5 \mathrm{~Hz}, 2 \mathrm{H}, \mathrm{CH}_{2}\right), 3.86\left(\mathrm{~s}, 3 \mathrm{H}, \mathrm{CH}_{3}\right), 4.04\left(\mathrm{t}, J=6.2 \mathrm{~Hz}, 2 \mathrm{H}, \mathrm{CH}_{2}\right), 6.93(\mathrm{dd}$, $J=7.9,2.7 \mathrm{~Hz}, 1 \mathrm{H}, \mathrm{Ar}), 7.09(\mathrm{t}, J=2.7 \mathrm{~Hz}, 1 \mathrm{H}, \mathrm{Ar}), 7.14-7.17$ (m, 1H, Ar), 7.21 (br s, NH), 7.24 (d, $J=1.7 \mathrm{~Hz}, 1 \mathrm{H}, \mathrm{Ar}), 7.36$ (t, $J=7.9 \mathrm{~Hz}, 1 \mathrm{H}, \mathrm{Ar}), 7.44$ $(\mathrm{dd}, J=8.5,1.7 \mathrm{~Hz}, 1 \mathrm{H}, \mathrm{Ar}), 8.27(\mathrm{~d}, J=8.5 \mathrm{~Hz}, 1 \mathrm{H}, \mathrm{Ar}) ;{ }^{13} \mathrm{C}-\mathrm{NMR}(100 \mathrm{MHz}$, $\left.\mathrm{CDCl}_{3}\right) \delta:$ 21.0, 43.8, 45.0, 55.4, 112.8, 113.6, 119.5, 121.8, 125.2, 125.6, 129.3, 129.3, 129.9, 140.6, 143.4, 146.4, 153.4, 160.0; Anal. calcd for $\mathrm{C}_{18} \mathrm{H}_{17} \mathrm{~N}_{3} \mathrm{OS}$ : C, 66.85; H, 5.30; N, 12.99. Found: C, 66.56; H, 5.14; N, 12.83.

\subsubsection{Synthesis of 3,4-Dihydro-9-(3-isopropoxyphenyl)- 2H,6H-pyrimido[1,2-c][1,3]benzothiazin-6-imine (31q)}

$N$-(tert-Butyl)-3,4-dihydro-9-(3-isopropoxyphenyl)-2H,6H-pyrimido[1,2-c] [1, 3]benzothiazin-6-imine (29q). Using the general procedure as described for 22l, $\mathrm{N}$-(tert-butyl)-9-bromo-3,4-dihydro-2 $\mathrm{H}, 6 \mathrm{H}$-pyrimido[1,2-c][1,3]benzothiazin-6imine 22k $(52.8 \mathrm{mg}, 0.15 \mathrm{mmol})$ was allowed to react with 3-isopropoxyphenylboronic acid $(32.4 \mathrm{mg}, 0.18 \mathrm{mmol})$ for $1 \mathrm{~h}$. Purification by flash chromatography over aluminum oxide with $n$-hexane-EtOAc (1:0 to 9:1) gave the title compound $\mathbf{2 9 q}$ as a colorless oil $(48.8 \mathrm{mg}, 80 \%)$ : IR (neat) $\mathrm{cm}^{-1}: 1592$ $(\mathrm{C}=\mathrm{N}) ;{ }^{1} \mathrm{H}-\mathrm{NMR}\left(500 \mathrm{MHz}, \mathrm{CDCl}_{3}\right) \delta: 1.36\left(\mathrm{~d}, J=5.7 \mathrm{~Hz}, 6 \mathrm{H}, 2 \times \mathrm{CH}_{3}\right), 1.40$ $\left(\mathrm{s}, 9 \mathrm{H}, 3 \times \mathrm{CH}_{3}\right), 1.90-1.95\left(\mathrm{~m}, 2 \mathrm{H}, \mathrm{CH}_{2}\right), 3.64\left(\mathrm{t}, J=5.7 \mathrm{~Hz}, 2 \mathrm{H}, \mathrm{CH}_{2}\right), 3.89$ (t, $\left.J=6.0 \mathrm{~Hz}, 2 \mathrm{H}, \mathrm{CH}_{2}\right), 4.58-4.65(\mathrm{~m}, 1 \mathrm{H}, \mathrm{CH}), 6.89(\mathrm{dd}, J=8.0,2.0 \mathrm{~Hz}, 1 \mathrm{H}, \mathrm{Ar})$, $7.10(\mathrm{t}, J=2.0 \mathrm{~Hz}, 1 \mathrm{H}, \mathrm{Ar}), 7.14(\mathrm{dd}, J=7.4,2.0 \mathrm{~Hz}, 1 \mathrm{H}, \mathrm{Ar}), 7.31-7.34(\mathrm{~m}$, 2H, Ar), 7.41 (dd, $J=8.0,1.7 \mathrm{~Hz}, 1 \mathrm{H}, \mathrm{Ar}), 8.24(\mathrm{~d}, J=8.0 \mathrm{~Hz}, 1 \mathrm{H}, \mathrm{Ar}) ;{ }^{13} \mathrm{C}-$ NMR (125 MHz, $\mathrm{CDCl}_{3}$ ) $\delta:$ 21.9, 22.0 (2C), 30.0 (3C), 45.1, 45.4, 54.1, 69.9, $114.9,115.1,119.3,122.8,124.8,126.6,128.8,129.4,129.9,138.3,140.9,142.8$, 147.7, 158.3; HRMS (FAB): $m / z$ calcd for $\mathrm{C}_{24} \mathrm{H}_{30} \mathrm{~N}_{3} \mathrm{OS}[\mathrm{M}+\mathrm{H}]^{+}$408.2110; found: 408.2108 .

Compound 31q. Using the general procedure as described for 25a, compound 29q (44.0 mg, $0.108 \mathrm{mmol}$ ) was allowed to react for $2 \mathrm{~h}$. Purification by flash chromatography over aluminum oxide with $n$-hexane-EtOAc $(9: 1$ to $7: 3)$ gave the title compound $\mathbf{3 1 q}$ as a colorless oil $\left(35.5 \mathrm{mg}, 94 \%\right.$ ): IR (neat) $\mathrm{cm}^{-1}$ : 1620 $(\mathrm{C}=\mathrm{N}), 1567(\mathrm{C}=\mathrm{N}) ;{ }^{1} \mathrm{H}-\mathrm{NMR}\left(400 \mathrm{MHz}, \mathrm{CDCl}_{3}\right) \delta: 1.36(\mathrm{~d}, J=6.1 \mathrm{~Hz}, 6 \mathrm{H}$, $\left.2 \times \mathrm{CH}_{3}\right), 1.95-2.01\left(\mathrm{~m}, 2 \mathrm{H}, \mathrm{CH}_{2}\right), 3.70\left(\mathrm{t}, J=5.5 \mathrm{~Hz}, 2 \mathrm{H}, \mathrm{CH}_{2}\right), 4.03(\mathrm{t}$, $\left.J=6.2 \mathrm{~Hz}, 2 \mathrm{H}, \mathrm{CH}_{2}\right), 4.57-4.66(\mathrm{~m}, 1 \mathrm{H}, \mathrm{CH}), 6.90(\mathrm{dd}, J=8.3,2.4 \mathrm{~Hz}, 1 \mathrm{H}, \mathrm{Ar})$, 7.09 (d, $J=1.8 \mathrm{~Hz}, 1 \mathrm{H}, \mathrm{Ar}), 7.11-7.13$ (m, 1H, Ar), 7.23 (d, $J=1.8 \mathrm{~Hz}, 1 \mathrm{H}, \mathrm{Ar}$ ), $7.33(\mathrm{t}, J=8.0 \mathrm{~Hz}, 1 \mathrm{H}, \mathrm{Ar}), 7.43(\mathrm{dd}, J=8.5,1.8 \mathrm{~Hz}, 1 \mathrm{H}, \mathrm{Ar}), 8.26(\mathrm{~d}$, $J=8.5 \mathrm{~Hz}, 1 \mathrm{H}, \mathrm{Ar}) ;{ }^{13} \mathrm{C}-\mathrm{NMR}\left(100 \mathrm{MHz}, \mathrm{CDCl}_{3}\right) \delta: 21.0,22.0$ (2C), 43.8, 45.0, $70.0,115.0,115.2,119.2,121.8,125.1,125.5,129.2,129.3,129.9,140.6,143.4$, 146.4, 153.4, 158.3; HRMS (FAB): $m / z$ calcd for $\mathrm{C}_{20} \mathrm{H}_{22} \mathrm{~N}_{3} \mathrm{OS}[\mathrm{M}+\mathrm{H}]^{+}$ 352.1484; found: 352.1484 . 


\subsubsection{Synthesis of 9-[(1,1'-Biphenyl)-3-yl]-3,4-dihydro- 2H,6H-pyrimido[1,2-c][1,3]benzothiazin-6-imine $(31 r)$}

9-[(1,1'-Biphenyl)-3-yl]- $N$-(tert-butyl)-3,4-dihydro-2H,6H-pyrimido[1,2-c][1,3] benzothiazin-6-imine (29r). Using the general procedure as described for 221 , $\mathrm{N}$-(tert-butyl)-9-bromo-3,4-dihydro-2H,6H-pyrimido[1,2-c][1,3]benzothiazin-6imine 22k ( $52.8 \mathrm{mg}, 0.15 \mathrm{mmol})$ was allowed to react with 3-biphenylboronic acid ( $35.7 \mathrm{mg}, 0.18 \mathrm{mmol}$ ) for $1 \mathrm{~h}$. Purification by flash chromatography over aluminum oxide with $n$-hexane-EtOAc (1:0 to 9:1) gave the title compound $29 \mathrm{r}$ as a colorless oil $\left(65.1 \mathrm{mg},>99 \%\right.$.): IR (neat) $\mathrm{cm}^{-1}$ : $1591 \quad(\mathrm{C}=\mathrm{N}) ;{ }^{1} \mathrm{H}-\mathrm{NMR}$ $\left(500 \mathrm{MHz}, \mathrm{CDCl}_{3}\right) \delta: 1.41\left(\mathrm{~s}, 9 \mathrm{H}, 3 \times \mathrm{CH}_{3}\right), 1.91-1.95\left(\mathrm{~m}, 2 \mathrm{H}, \mathrm{CH}_{2}\right), 3.64(\mathrm{t}$, $\left.J=5.4 \mathrm{~Hz}, 2 \mathrm{H}, \mathrm{CH}_{2}\right), 3.89\left(\mathrm{t}, J=6.3 \mathrm{~Hz}, 2 \mathrm{H}, \mathrm{CH}_{2}\right), 7.35-7.39(\mathrm{~m}, 2 \mathrm{H}, \mathrm{Ar})$, 7.44-7.51 (m, 4H, Ar), 7.55-7.60 (m, 2H, Ar), 7.63 (d, J=7.4 Hz, 2H, Ar), 7.78 (s, $1 \mathrm{H}, \mathrm{Ar}), 8.27(\mathrm{~d}, J=8.0 \mathrm{~Hz}, 1 \mathrm{H}, \mathrm{Ar}) ;{ }^{13} \mathrm{C}-\mathrm{NMR}\left(125 \mathrm{MHz}, \mathrm{CDCl}_{3}\right) \delta: 21.9$, 30.0 (3C), 45.1, 45.4, 54.2, 122.8, 124.9, 125.9 (2C), 126.7, 126.9, 127.2 (2C), 127.5, 128.8 (2C), 129.0, 129.3, 129.6, 138.2, 139.9, 140.9, 142.0, 142.8, 147.7; HRMS (FAB): $m / z$ calcd for $\mathrm{C}_{27} \mathrm{H}_{28} \mathrm{~N}_{3} \mathrm{~S}[\mathrm{M}+\mathrm{H}]^{+}$426.2004; found: 426.2000.

Compound 31r. Using the general procedure as described for 25a, compound 29r $(56.1 \mathrm{mg}, 0.13 \mathrm{mmol})$ was allowed to react for $3 \mathrm{~h}$. Purification by flash chromatography over aluminum oxide with $n$-hexane-EtOAc $(9: 1$ to $7: 3)$ gave the title compound 31r as colorless solid (37.0 mg, $76 \%$ ): $\mathrm{mp} 179-181{ }^{\circ} \mathrm{C}$ (from $\mathrm{CHCl}_{3}-n$-hexane); IR (neat) $\mathrm{cm}^{-1}: 1620 \quad(\mathrm{C}=\mathrm{N}), 1568 \quad(\mathrm{C}=\mathrm{N}) ;{ }^{1} \mathrm{H}-\mathrm{NMR}$ $\left(500 \mathrm{MHz} \mathrm{CDCl}_{3}\right) \delta: 1.97-2.02\left(\mathrm{~m}, 2 \mathrm{H}, \mathrm{CH}_{2}\right), 3.72\left(\mathrm{t}, J=5.4 \mathrm{~Hz}, 2 \mathrm{H}, \mathrm{CH}_{2}\right)$, $4.04\left(\mathrm{t}, J=6.0 \mathrm{~Hz}, 2 \mathrm{H}, \mathrm{CH}_{2}\right), 7.30(\mathrm{~d}, J=1.7 \mathrm{~Hz}, 1 \mathrm{H}, \mathrm{Ar}), 7.38$ (t, $J=7.4 \mathrm{~Hz}$, 1H, Ar), 7.45-7.56 (m, 5H, Ar), 7.59-7.64 (m, 3H, Ar), 7.77 (s, 1H, Ar), 8.30 (d, $J=8.6 \mathrm{~Hz}, 1 \mathrm{H}, \mathrm{Ar}) ;{ }^{13} \mathrm{C}-\mathrm{NMR}\left(125 \mathrm{MHz}, \mathrm{CDCl}_{3}\right) \delta: 21.0,43.9,45.0,121.9$, 125.2, 125.6, 125.9 (2C), 127.1, 127.2 (2C), 127.6, 128.8 (2C), 129.3, 129.4 (2C), 139.7, 140.8, 142.1, 143.4, 146.4, 153.3; HRMS (FAB): $m / z$ calcd for $\mathrm{C}_{23} \mathrm{H}_{20} \mathrm{~N}_{3} \mathrm{~S}$ $[\mathrm{M}+\mathrm{H}]^{+} 370.1378$; found: 370.1378 .

\subsubsection{Synthesis of 3,4-Dihydro-9-(2-methoxyphenyl)- 2H,6H-pyrimido[1,2-c][1,3]benzothiazin-6-imine $(31 s)$}

$N$-(tert-Butyl)-3,4-dihydro-9-(2-methoxyphenyl)-2H,6H-pyrimido[1,2-c][1,3] benzothiazin-6-imine (29s). Using the general procedure as described for 22l, $N$-(tert-butyl)-9-bromo-3,4-dihydro-2 $H, 6 H$-pyrimido[1,2-c][1,3]benzothiazin6-imine $22 \mathbf{k}(52.8 \mathrm{mg}, 0.15 \mathrm{mmol})$ was allowed to react with 2-methoxyphenylboronic acid $(27.4 \mathrm{mg}, 0.18 \mathrm{mmol})$ for $1 \mathrm{~h}$. Purification by flash chromatography over aluminum oxide with $n$-hexane-EtOAc (1:0 to 9:1) gave the title 
compound 29s as a colorless oil $(46.0 \mathrm{mg}, 81 \%)$ : IR (neat) $\mathrm{cm}^{-1}$ : $1591(\mathrm{C}=\mathrm{N})$; ${ }^{1} \mathrm{H}-\mathrm{NMR}\left(400 \mathrm{MHz}, \mathrm{CDCl}_{3}\right) \delta: 1.39\left(\mathrm{~s}, 9 \mathrm{H}, 3 \times \mathrm{CH}_{3}\right), 1.89-1.95\left(\mathrm{~m}, 2 \mathrm{H}, \mathrm{CH}_{2}\right)$, $3.63\left(\mathrm{t}, J=5.4 \mathrm{~Hz}, 2 \mathrm{H}, \mathrm{CH}_{2}\right), 3.80\left(\mathrm{~s}, 3 \mathrm{H}, \mathrm{CH}_{3}\right), 3.88\left(\mathrm{t}, J=5.9 \mathrm{~Hz}, 2 \mathrm{H}, \mathrm{CH}_{2}\right)$, $6.97(\mathrm{~d}, J=8.3 \mathrm{~Hz}, 1 \mathrm{H}, \mathrm{Ar}), 7.02(\mathrm{t}, J=7.4 \mathrm{~Hz}, 1 \mathrm{H}, \mathrm{Ar}), 7.29-7.38(\mathrm{~m}, 4 \mathrm{H}, \mathrm{Ar})$, $8.21(\mathrm{~d}, J=8.3 \mathrm{~Hz}, 1 \mathrm{H}, \mathrm{Ar}) ;{ }^{13} \mathrm{C}-\mathrm{NMR}\left(100 \mathrm{MHz}, \mathrm{CDCl}_{3}\right) \delta: 22.0,30.0(3 \mathrm{C})$, $45.1,45.4,54.1,55.6,111.4,120.9,125.2$, 126.2, 127.5, 128.0, 128.6, 129.1, 129.3, 130.6, 138.6, 140.6, 147.9, 156.5; HRMS (FAB): $\mathrm{m} / \mathrm{z}$ calcd for $\mathrm{C}_{22} \mathrm{H}_{26} \mathrm{~N}_{3} \mathrm{OS}[\mathrm{M}+\mathrm{H}]^{+}$380.1797; found: 380.1793 .

Compound 31s. Using the general procedure as described for 25a, compound 29s $(34.7 \mathrm{mg}, 0.091 \mathrm{mmol}$ ) was allowed to react for $2 \mathrm{~h}$. Purification by flash chromatography over aluminum oxide with $n$-hexane-EtOAc (9:1 to 7:3) gave the title compound 31s as colorless solid $\left(21.6 \mathrm{mg}, 73 \%\right.$ ): $\mathrm{mp} 130.5^{\circ} \mathrm{C}$ (from $\mathrm{CHCl}_{3}-n$-hexane); IR (neat) $\mathrm{cm}^{-1}: 1620(\mathrm{C}=\mathrm{N}), 1567(\mathrm{C}=\mathrm{N}) ;{ }^{1} \mathrm{H}-\mathrm{NMR}$ $\left(400 \mathrm{MHz}, \mathrm{CDCl}_{3}\right) \delta: 1.95-2.01\left(\mathrm{~m}, 2 \mathrm{H}, \mathrm{CH}_{2}\right), 3.70\left(\mathrm{t}, J=5.6 \mathrm{~Hz}, 2 \mathrm{H}, \mathrm{CH}_{2}\right), 3.80$ $\left(\mathrm{s}, 3 \mathrm{H}, \mathrm{CH}_{3}\right), 4.03\left(\mathrm{t}, J=6.2 \mathrm{~Hz}, 2 \mathrm{H}, \mathrm{CH}_{2}\right), 6.97(\mathrm{~d}, J=8.3 \mathrm{~Hz}, 1 \mathrm{H}, \mathrm{Ar}), 7.02$ (td, $J=7.5,0.8 \mathrm{~Hz}, 1 \mathrm{H}, \mathrm{Ar}), 7.22(\mathrm{~d}, J=1.5 \mathrm{~Hz}, 1 \mathrm{H}, \mathrm{Ar}), 7.28-7.40(\mathrm{~m}, 3 \mathrm{H}, \mathrm{Ar})$, $8.24(\mathrm{~d}, J=8.3 \mathrm{~Hz}, 1 \mathrm{H}, \mathrm{Ar}) ;{ }^{13} \mathrm{C}-\mathrm{NMR}\left(100 \mathrm{MHz}, \mathrm{CDCl}_{3}\right) \delta: 21.1,43.8,44.9$, 55.6, 111.3, 120.9, 124.3, 125.1, 127.7, 128.3, 128.4, 128.7, 129.5, 130.5, 141.1, 146.6, 153.7, 156.4; Anal. calcd for $\mathrm{C}_{18} \mathrm{H}_{17} \mathrm{~N}_{3} \mathrm{OS}$ : C, 66.85; H, 5.30; N, 12.99 . Found: C, 66.56; H, 5.08; N, 12.90 .

\subsubsection{Synthesis of 9-[(1,1'-Biphenyl)-2-yl]-3,4-dihydro- 2H,6H-pyrimido[1,2-c][1,3]benzothiazin-6-imine (31t)}

9-[(1,1'-Biphenyl)-2-yl]- $N$-(tert-butyl)-3,4-dihydro-2H,6H-pyrimido[1,2-c][1,3] benzothiazin-6-imine (29t). Using the general procedure as described for 22l, $N$-(tert-butyl)-9-bromo-3,4-dihydro- $2 \mathrm{H}, 6 \mathrm{H}$-pyrimido[1,2-c][1,3]benzothiazin6-imine 22k (52.8 mg, $0.15 \mathrm{mmol})$ was allowed to react with 2-biphenylboronic acid $(35.7 \mathrm{mg}, 0.18 \mathrm{mmol}$ ) for $1 \mathrm{~h}$. Purification by flash chromatography over aluminum oxide with $n$-hexane-EtOAc (1:0 to 9:1) gave the title compound 29t as a colorless oil $\left(64.1 \mathrm{mg},>99 \%\right.$.): IR (neat) $\mathrm{cm}^{-1}$ : $1591 \quad(\mathrm{C}=\mathrm{N}) ;{ }^{1} \mathrm{H}-\mathrm{NMR}$ $\left(400 \mathrm{MHz}, \mathrm{CDCl}_{3}\right) \delta: 1.36\left(\mathrm{~s}, 9 \mathrm{H}, 3 \times \mathrm{CH}_{3}\right), 1.86-1.91\left(\mathrm{~m}, 2 \mathrm{H}, \mathrm{CH}_{2}\right), 3.59(\mathrm{t}$, $\left.J=5.6 \mathrm{~Hz}, 2 \mathrm{H}, \mathrm{CH}_{2}\right), 3.85\left(\mathrm{t}, J=6.2 \mathrm{~Hz}, 2 \mathrm{H}, \mathrm{CH}_{2}\right), 6.91-6.93(\mathrm{~m}, 2 \mathrm{H}, \mathrm{Ar})$, 7.11-7.14 (m, 2H, Ar), 7.19-7.22 (m, 3H, Ar), 7.40-7.42 (m, 4H, Ar), 7.99 (d, $J=8.8 \mathrm{~Hz}, 1 \mathrm{H}, \mathrm{Ar}) ;{ }^{13} \mathrm{C}-\mathrm{NMR}\left(100 \mathrm{MHz} \mathrm{CDCl}_{3}\right) \delta: 21.9,29.9$ (3C), 45.1, 45.4, $54.1,125.5,125.9,126.7,127.5,127.9,127.9,128.1,128.1$ (2C), 128.6, 129.7 (2C), 130.2, 130.7, 138.4, 138.9, 140.6, 140.9, 143.6, 147.8; HRMS (FAB): $m / z$ calcd for $\mathrm{C}_{27} \mathrm{H}_{28} \mathrm{~N}_{3} \mathrm{~S}[\mathrm{M}+\mathrm{H}]^{+}$426.2004; found: 426.2002.

Compound 31t. Using the general procedure as described for $\mathbf{2 5 a}$, compound 29t $(51.1 \mathrm{mg}, 0.12 \mathrm{mmol})$ was allowed to react for $3 \mathrm{~h}$. Purification by flash chromatography over aluminum oxide with $n$-hexane-EtOAc $(9: 1$ to $7: 3)$ gave the title compound $\mathbf{3 1 t}$ as a colorless oil $(35.7 \mathrm{mg}, 81 \%)$ : IR (neat) $\mathrm{cm}^{-1}$ : 1620 
$(\mathrm{C}=\mathrm{N}), 1570(\mathrm{C}=\mathrm{N}) ;{ }^{1} \mathrm{H}-\mathrm{NMR}\left(400 \mathrm{MHz}, \mathrm{CDCl}_{3}\right) \delta: 1.92-1.98\left(\mathrm{~m}, 2 \mathrm{H}, \mathrm{CH}_{2}\right), 3.66$ $\left(\mathrm{t}, J=5.5 \mathrm{~Hz}, 2 \mathrm{H}, \mathrm{CH}_{2}\right), 3.99\left(\mathrm{t}, J=6.1 \mathrm{~Hz}, 2 \mathrm{H}, \mathrm{CH}_{2}\right), 6.83(\mathrm{~d}, J=1.7 \mathrm{~Hz}, 1 \mathrm{H}$, Ar), 6.95 (dd, $J=8.3,1.7 \mathrm{~Hz}, 1 \mathrm{H}, \mathrm{Ar}), 7.11-7.13$ (m, 2H, Ar), 7.20-7.23 (m, 3H, Ar), 7.38-7.43 (m, 4H, Ar), 8.03 (d, $J=8.3 \mathrm{~Hz}, 1 \mathrm{H}, \mathrm{Ar}) ;{ }^{13} \mathrm{C}-\mathrm{NMR}(100 \mathrm{MHz}$, $\left.\mathrm{CDCl}_{3}\right) \delta: 21.0,43.8,44.9,124.5,124.8,126.8,127.6,128.1$ (3C), 128.1, 128.2, 128.3, 128.4, 129.7 (2C), 130.2, 138.5, 140.6, 140.8, 144.2, 146.5, 153.5; HRMS (FAB): $m / z$ calcd for $\mathrm{C}_{23} \mathrm{H}_{20} \mathrm{~N}_{3} \mathrm{~S}[\mathrm{M}+\mathrm{H}]^{+} 370.1378$; found: 370.1378 .

\subsubsection{Synthesis of 3,4-Dihydro-9-(3,4-dimethoxyphenyl)- 2H,6H-pyrimido[1,2-c][1,3]benzothiazin-6-imine (31u)}

$N$-(tert-Butyl)-3,4-dihydro-9-(3,4-dimethoxyphenyl)-2H,6H-pyrimido[1,2-c] [1,3]benzothiazin-6-imine $(\mathbf{2 9 u})$. Using the general procedure as described for 22l, $N$-(tert-butyl)-9-bromo-3,4-dihydro-2H,6H-pyrimido[1,2-c][1,3]benzothiazin6-imine $22 \mathrm{k}(52.8 \mathrm{mg}, 0.15 \mathrm{mmol})$ was allowed to react with 3,4-dimethoxyphenylboronic acid $(32.8 \mathrm{mg}, 0.18 \mathrm{mmol})$ for $1 \mathrm{~h}$. Purification by flash chromatography over aluminum oxide with $n$-hexane-EtOAc (1:0 to 9:1) gave the title compound 29u as colorless solid $\left(60.3 \mathrm{mg}, 98 \%\right.$ ): $\mathrm{mp} 147-148{ }^{\circ} \mathrm{C}$ (from $\mathrm{CHCl}_{3}-n$-hexane); IR (neat) $\mathrm{cm}^{-1}: 1593(\mathrm{C}=\mathrm{N}) ;{ }^{1} \mathrm{H}-\mathrm{NMR}\left(400 \mathrm{MHz}, \mathrm{CDCl}_{3}\right) \delta$ : $1.40\left(\mathrm{~s}, 9 \mathrm{H}, 3 \times \mathrm{CH}_{3}\right), 1.90-1.96\left(\mathrm{~m}, 2 \mathrm{H}, \mathrm{CH}_{2}\right), 3.64\left(\mathrm{t}, J=5.5 \mathrm{~Hz}, 2 \mathrm{H}, \mathrm{CH}_{2}\right)$, $3.89\left(\mathrm{t}, J=6.1 \mathrm{~Hz}, 2 \mathrm{H}, \mathrm{CH}_{2}\right), 3.92\left(\mathrm{~s}, 3 \mathrm{H}, \mathrm{CH}_{3}\right), 3.96\left(\mathrm{~s}, 3 \mathrm{H}, \mathrm{CH}_{3}\right), 6.93(\mathrm{~d}$, $J=8.3 \mathrm{~Hz}, 1 \mathrm{H}, \mathrm{Ar}), 7.09(\mathrm{~d}, J=2.0 \mathrm{~Hz}, 1 \mathrm{H}, \mathrm{Ar}), 7.15(\mathrm{dd}, J=8.3,2.0 \mathrm{~Hz}, 1 \mathrm{H}$, Ar), $7.29(\mathrm{~d}, J=1.7 \mathrm{~Hz}, 1 \mathrm{H}, \mathrm{Ar}), 7.39(\mathrm{dd}, J=8.3,1.7 \mathrm{~Hz}, 1 \mathrm{H}, \mathrm{Ar}), 8.23$ (d, $J=8.3 \mathrm{~Hz}, 1 \mathrm{H}, \mathrm{Ar}) ;{ }^{13} \mathrm{C}-\mathrm{NMR}\left(125 \mathrm{MHz}, \mathrm{CDCl}_{3}\right) \delta: 21.9,30.0$ (3C), 45.1, 45.4, $54.1,56.0,56.0,110.1,111.4,119.5,122.2,124.5,126.1,128.9,129.4,132.3$, 138.3, 142.7, 147.7, 149.3 (2C); HRMS (FAB): $m / z$ calcd for $\mathrm{C}_{23} \mathrm{H}_{28} \mathrm{~N}_{3} \mathrm{O}_{2} \mathrm{~S}$ $[\mathrm{M}+\mathrm{H}]^{+}$410.1902; found: 410.1907 .

Compound 31u. Using the general procedure as described for 25a, compound 29u (46.0 mg, $0.11 \mathrm{mmol}$ ) was allowed to react for $3 \mathrm{~h}$. Purification by flash chromatography over aluminum oxide with $n$-hexane-EtOAc $(9: 1$ to $7: 3)$ gave the title compound $31 \mathrm{u}$ as colorless solid $(24.6 \mathrm{mg}, 63 \%): \mathrm{mp} 142{ }^{\circ} \mathrm{C}$ (from $\mathrm{CHCl}_{3}$ $n$-hexane); IR (neat) $\mathrm{cm}^{-1}: 1620(\mathrm{C}=\mathrm{N}), 1567(\mathrm{C}=\mathrm{N}) ;{ }^{1} \mathrm{H}-\mathrm{NMR}$ (400 MHz, $\left.\mathrm{CDCl}_{3}\right) \delta: 1.96-2.02\left(\mathrm{~m}, 2 \mathrm{H}, \mathrm{CH}_{2}\right), 3.71\left(\mathrm{t}, J=5.6 \mathrm{~Hz}, 2 \mathrm{H}, \mathrm{CH}_{2}\right), 3.92(\mathrm{~s}, 3 \mathrm{H}$, $\left.\mathrm{CH}_{3}\right), 3.96\left(\mathrm{~s}, 3 \mathrm{H}, \mathrm{CH}_{3}\right), 4.04\left(\mathrm{t}, J=6.2 \mathrm{~Hz}, 2 \mathrm{H}, \mathrm{CH}_{2}\right), 6.93(\mathrm{~d}, J=8.3 \mathrm{~Hz}, 1 \mathrm{H}$, Ar), $7.07(\mathrm{~d}, J=2.0 \mathrm{~Hz}, 1 \mathrm{H}, \mathrm{Ar}), 7.14(\mathrm{dd}, J=8.3,2.0 \mathrm{~Hz}, 1 \mathrm{H}, \mathrm{Ar}), 7.20$ (d, $J=1.8 \mathrm{~Hz}, 1 \mathrm{H}, \mathrm{Ar}), 7.41(\mathrm{dd}, J=8.3,1.8 \mathrm{~Hz}, 1 \mathrm{H}, \mathrm{Ar}), 8.25(\mathrm{~d}, J=8.3 \mathrm{~Hz}, 1 \mathrm{H}$, Ar); ${ }^{13} \mathrm{C}-\mathrm{NMR}\left(100 \mathrm{MHz}, \mathrm{CDCl}_{3}\right) \delta: 21.0,43.8,44.9,56.0,56.0,110.1,111.5$, 119.5, 121.3, 124.7, 125.0, 129.3, 129.4, 132.0, 143.2, 146.4, 149.3, 149.4, 153.4; Anal. calcd for $\mathrm{C}_{19} \mathrm{H}_{19} \mathrm{~N}_{3} \mathrm{O}_{2} \mathrm{~S}$ : C, 64.57; H, 5.42; N, 11.89. Found: C, 64.41; H, 5.37; N, 11.93 . 


\subsubsection{Synthesis of 3,4-Dihydro-9-(3,4,5-trimethoxyphenyl)- 2H,6H-pyrimido[1,2-c][1,3]benzothiazin-6-imine (31v)}

$N$-(tert-Butyl)-3,4-dihydro-9-(3,4,5-trimethoxyphenyl)-2H,6H-pyrimido[1,2-c] [1,3]benzothiazin-6-imine $(\mathbf{2 9} \mathrm{v})$. Using the general procedure as described for 22l, $\mathrm{N}$-(tert-butyl)-9-bromo-3,4-dihydro-2H,6H-pyrimido[1,2-c][1,3]benzothiazin6-imine $22 \mathbf{k}(52.8 \mathrm{mg}, 0.15 \mathrm{mmol})$ was allowed to react with 3,4,5-trimethoxyphenylboronic acid $(38.2 \mathrm{mg}, 0.18 \mathrm{mmol})$ for $1 \mathrm{~h}$. Purification by flash chromatography over aluminum oxide with $n$-hexane-EtOAc (1:0 to 9:1) gave the title compound $29 \mathrm{v}$ as a colorless oil $(65.0 \mathrm{mg}, 99 \%)$ : IR (neat) $\mathrm{cm}^{-1}: 1585$ $(\mathrm{C}=\mathrm{N}) ;{ }^{1} \mathrm{H}-\mathrm{NMR}\left(400 \mathrm{MHz}, \mathrm{CDCl}_{3}\right) \delta: 1.41\left(\mathrm{~s}, 9 \mathrm{H}, 3 \times \mathrm{CH}_{3}\right), 1.91-1.96(\mathrm{~m}, 2 \mathrm{H}$, $\left.\mathrm{CH}_{2}\right), 3.64\left(\mathrm{t}, J=5.5 \mathrm{~Hz}, 2 \mathrm{H}, \mathrm{CH}_{2}\right), 3.88-3.91\left(\mathrm{~m}, 5 \mathrm{H}, \mathrm{CH}_{3}, \mathrm{CH}_{2}\right), 3.93(\mathrm{~s}, 6 \mathrm{H}$, $\left.2 \times \mathrm{CH}_{3}\right), 6.77(\mathrm{~s}, 2 \mathrm{H}, \mathrm{Ar}), 7.28(\mathrm{~d}, J=1.8 \mathrm{~Hz}, 1 \mathrm{H}, \mathrm{Ar}), 7.38(\mathrm{dd}, J=8.4$, $1.8 \mathrm{~Hz}, 1 \mathrm{H}, \mathrm{Ar}), 8.24$ (d, $J=8.4 \mathrm{~Hz}, 1 \mathrm{H}, \mathrm{Ar}) ;{ }^{13} \mathrm{C}-\mathrm{NMR}\left(100 \mathrm{MHz}, \mathrm{CDCl}_{3}\right) \delta$ : 21.9, 30.0 (3C), 45.1, 45.4, 54.2, 56.3 (2C), 60.9, 104.4 (2C), 122.6, 124.7, 126.5, 128.9, 129.5, 135.3, 138.1, 138.3, 143.0, 147.7, 153.6 (2C); HRMS (FAB): $m / z$ calcd for $\mathrm{C}_{24} \mathrm{H}_{30} \mathrm{~N}_{3} \mathrm{O}_{3} \mathrm{~S}[\mathrm{M}+\mathrm{H}]^{+}$440.2008; found: 440.2008 .

Compound 31v. Using the general procedure as described for 25a, compound 29v (49.4 mg, $0.11 \mathrm{mmol}$ ) was allowed to react for $3 \mathrm{~h}$. Purification by flash chromatography over aluminum oxide with $n$-hexane-EtOAc $(9: 1$ to $7: 3)$ gave the title compound $\mathbf{3 1 v}$ as colorless solid $\left(14.2 \mathrm{mg}, 34 \%\right.$ ): $\mathrm{mp} 156-157{ }^{\circ} \mathrm{C}$ (from $\mathrm{CHCl}_{3}-n$-hexane); IR (neat) $\mathrm{cm}^{-1}: 1620 \quad(\mathrm{C}=\mathrm{N}), 1569 \quad(\mathrm{C}=\mathrm{N}) ;{ }^{1} \mathrm{H}-\mathrm{NMR}$ $\left(400 \mathrm{MHz} \mathrm{CDCl}_{3}\right) \delta: 1.97-2.02\left(\mathrm{~m}, 2 \mathrm{H}, \mathrm{CH}_{2}\right), 3.71\left(\mathrm{t}, J=5.5 \mathrm{~Hz}, 2 \mathrm{H}, \mathrm{CH}_{2}\right)$, $3.89\left(\mathrm{~s}, 3 \mathrm{H}, \mathrm{CH}_{3}\right), 3.93\left(\mathrm{~s}, 6 \mathrm{H}, 2 \times \mathrm{CH}_{3}\right), 4.04\left(\mathrm{t}, J=6.1 \mathrm{~Hz}, 2 \mathrm{H}, \mathrm{CH}_{2}\right), 6.75(\mathrm{~s}$, $2 \mathrm{H}, \mathrm{Ar}), 7.20$ (d, $J=1.7 \mathrm{~Hz}, 1 \mathrm{H}, \mathrm{Ar}), 7.40(\mathrm{dd}, J=8.5,1.7 \mathrm{~Hz}, 1 \mathrm{H}, \mathrm{Ar}), 8.27$ (d, $J=8.5 \mathrm{~Hz}, 1 \mathrm{H}, \mathrm{Ar}) ;{ }^{13} \mathrm{C}-\mathrm{NMR}\left(100 \mathrm{MHz}, \mathrm{CDCl}_{3}\right) \delta: 21.0,43.9,45.0,56.3$ (2C), $61.0,104.4$ (2C), 121.7, 125.0, 125.4, 129.3 (2C), 135.0, 138.5, 143.6, 146.4, 153.3, 153.6 (2C); HRMS (FAB): $m / z$ calcd for $\mathrm{C}_{20} \mathrm{H}_{22} \mathrm{~N}_{3} \mathrm{O}_{3} \mathrm{~S}[\mathrm{M}+\mathrm{H}]^{+}$ 384.1382; found: 384.1381 .

\subsubsection{Synthesis of 9-(3-Chloro-4-methoxyphenyl)-3,4- dihydro-2H,6H-pyrimido[1,2-c][1,3]benzothiazin-6 -imine (31w)}

$\mathrm{N}$-(ter $\boldsymbol{t}$-Butyl)-9-(3-chloro-4-methoxyphenyl)-3,4-dihydro-2H,6H-pyrimido[1, 2-c][1,3]benzothiazin-6-imine (29w). Using the general procedure as described for 22l, $N$-(tert-butyl)-9-bromo-3,4-dihydro-2H,6H-pyrimido[1,2-c] [1,3]benzothiazin-6-imine $22 \mathbf{k}(52.8 \mathrm{mg}, 0.15 \mathrm{mmol})$ was allowed to react with 3-chloro-4methoxyphenylboronic acid $(33.6 \mathrm{mg}, 0.18 \mathrm{mmol})$ for $1 \mathrm{~h}$. Purification by flash chromatography over aluminum oxide with $n$-hexane-EtOAc (1:0 to 9:1) gave the 
title compound 29w as a colorless oil $(58.7 \mathrm{mg}, 95 \%)$ : IR (neat) $\mathrm{cm}^{-1}: 1592$ $(\mathrm{C}=\mathrm{N}) ;{ }^{1} \mathrm{H}-\mathrm{NMR}\left(500 \mathrm{MHz}, \mathrm{CDCl}_{3}\right) \delta: 1.40\left(\mathrm{~s}, 9 \mathrm{H}, 3 \times \mathrm{CH}_{3}\right), 1.90-1.95(\mathrm{~m}, 2 \mathrm{H}$, $\left.\mathrm{CH}_{2}\right), 3.64\left(\mathrm{t}, J=5.4 \mathrm{~Hz}, 2 \mathrm{H}, \mathrm{CH}_{2}\right), 3.89\left(\mathrm{t}, J=6.0 \mathrm{~Hz}, 2 \mathrm{H}, \mathrm{CH}_{2}\right), 3.94(\mathrm{~s}, 3 \mathrm{H}$, $\left.\mathrm{CH}_{3}\right), 6.98(\mathrm{~d}, J=8.6 \mathrm{~Hz}, 1 \mathrm{H}, \mathrm{Ar}), 7.26(\mathrm{~d}, J=2.0 \mathrm{~Hz}, 1 \mathrm{H}, \mathrm{Ar}), 7.36$ (dd, $J=8.6,2.0 \mathrm{~Hz}, 1 \mathrm{H}, \mathrm{Ar}), 7.45(\mathrm{dd}, J=8.6,2.3 \mathrm{~Hz}, 1 \mathrm{H}, \mathrm{Ar}), 7.61(\mathrm{~d}, J=2.3 \mathrm{~Hz}$, $1 \mathrm{H}, \mathrm{Ar}), 8.23$ (d, $J=8.6 \mathrm{~Hz}, 1 \mathrm{H}, \mathrm{Ar}) ;{ }^{13} \mathrm{C}-\mathrm{NMR}\left(125 \mathrm{MHz}, \mathrm{CDCl}_{3}\right) \delta: 21.9,30.0$ (3C), 45.1, 45.4, 54.2, 56.2, 112.2, 122.2, 123.0, 124.3, 126.2, 126.4, 128.7, 129.0, $129.7,132.7,138.1,141.2,147.6,155.0 ;$ HRMS (FAB): $\mathrm{m} / z$ calcd for $\mathrm{C}_{22} \mathrm{H}_{25} \mathrm{ClN}_{3} \mathrm{OS}[\mathrm{M}+\mathrm{H}]^{+}$414.1407; found: 414.1402.

Compound 31w. Using the general procedure as described for 25a, compound 29w (41.6 mg, $0.10 \mathrm{mmol}$ ) was allowed to react for $3 \mathrm{~h}$. Purification by flash chromatography over aluminum oxide with $n$-hexane-EtOAc $(9: 1$ to $7: 3)$ gave the title compound $\mathbf{3 1 w}$ as colorless solid $\left(18.8 \mathrm{mg}, 53 \%\right.$ ): $\mathrm{mp} 186-188{ }^{\circ} \mathrm{C}$ (from $\mathrm{CHCl}_{3}-n$-hexane); IR (neat) $\mathrm{cm}^{-1}: 1620 \quad(\mathrm{C}=\mathrm{N}), 1563 \quad(\mathrm{C}=\mathrm{N}) ;{ }^{1} \mathrm{H}-\mathrm{NMR}$ $\left(500 \mathrm{MHz}, \mathrm{CDCl}_{3}\right) \delta$ : 1.97-2.01 (m, 2H, $\left.\mathrm{CH}_{2}\right), 3.71\left(\mathrm{t}, J=5.4 \mathrm{~Hz}, 2 \mathrm{H}, \mathrm{CH}_{2}\right)$, $3.94\left(\mathrm{~s}, 3 \mathrm{H}, \mathrm{CH}_{3}\right), 4.03\left(\mathrm{t}, J=6.0 \mathrm{~Hz}, 2 \mathrm{H}, \mathrm{CH}_{2}\right), 6.98(\mathrm{~d}, J=8.6 \mathrm{~Hz}, 1 \mathrm{H}, \mathrm{Ar})$, $7.17(\mathrm{~d}, J=1.4 \mathrm{~Hz}, 1 \mathrm{H}, \mathrm{Ar}), 7.38(\mathrm{dd}, J=8.0,2.3 \mathrm{~Hz}, 1 \mathrm{H}, \mathrm{Ar}), 7.44$ (dd, $J=8.0,1.4 \mathrm{~Hz}, 1 \mathrm{H}, \mathrm{Ar}), 7.59(\mathrm{~d}, J=2.3 \mathrm{~Hz}, 1 \mathrm{H}, \mathrm{Ar}), 8.25(\mathrm{~d}, J=8.0 \mathrm{~Hz}, 1 \mathrm{H}$, $\mathrm{Ar}) ;{ }^{13} \mathrm{C}-\mathrm{NMR}\left(125 \mathrm{MHz}, \mathrm{CDCl}_{3}\right) \delta: 21.0,43.8,44.9,56.2,112.2,121.2,123.0$, 124.5, 125.3, 126.2, 128.7, 129.4, 129.5, 132.4, 141.7, 146.3, 153.2, 155.1; HRMS (FAB): $m / z$ calcd for $\mathrm{C}_{18} \mathrm{H}_{17} \mathrm{ClN}_{3} \mathrm{OS}[\mathrm{M}+\mathrm{H}]^{+}$358.0781; found: 358.0777 .

\subsubsection{Synthesis of 9-(3-Chloro-6-methoxyphenyl)- 3,4-dihydro-2H,6H-pyrimido[1,2-c][1,3]benzothiazin- 6-imine (31x)}

$\mathrm{N}$-(tert-Butyl)-9-(3-chloro-6-methoxyphenyl)-3,4-dihydro-2H,6H-pyrimido [1, 2-c][1,3]benzothiazin-6-imine (29x). Using the general procedure as described for 221, $N$-(tert-butyl)-9-bromo-3,4-dihydro- $2 H, 6 H$-pyrimido[1,2-c][1,3]benzothiazin-6-imine $22 \mathrm{k}(52.8 \mathrm{mg}, 0.15 \mathrm{mmol})$ was allowed to react with 3 -chloro-6methoxyphenylboronic acid $(33.6 \mathrm{mg}, 0.18 \mathrm{mmol})$ for $1 \mathrm{~h}$. Purification by flash chromatography over aluminum oxide with $n$-hexane-EtOAc (1:0 to 9:1) gave the title compound $29 x$ as a colorless oil $(54.9 \mathrm{mg}, 88 \%)$ : IR (neat) $\mathrm{cm}^{-1}: 1591$ $(\mathrm{C}=\mathrm{N}) ;{ }^{1} \mathrm{H}-\mathrm{NMR}\left(400 \mathrm{MHz}, \mathrm{CDCl}_{3}\right) \delta: 1.39\left(\mathrm{~s}, 9 \mathrm{H}, 3 \times \mathrm{CH}_{3}\right), 1.89-1.95(\mathrm{~m}, 2 \mathrm{H}$, $\left.\mathrm{CH}_{2}\right), 3.64\left(\mathrm{t}, J=5.5 \mathrm{~Hz}, 2 \mathrm{H}, \mathrm{CH}_{2}\right), 3.78\left(\mathrm{~s}, 3 \mathrm{H}, \mathrm{CH}_{3}\right), 3.88(\mathrm{t}, J=6.1 \mathrm{~Hz}, 2 \mathrm{H}$, $\left.\mathrm{CH}_{2}\right), 6.88$ (d, $\left.J=9.5 \mathrm{~Hz}, 1 \mathrm{H}, \mathrm{Ar}\right), 7.26-7.34(\mathrm{~m}, 4 \mathrm{H}, \mathrm{Ar}), 8.21$ (d, $J=8.3 \mathrm{~Hz}$, $1 \mathrm{H}, \mathrm{Ar}) ;{ }^{13} \mathrm{C}-\mathrm{NMR}\left(100 \mathrm{MHz}, \mathrm{CDCl}_{3}\right) \delta: 21.9,30.0$ (3C), 45.1, 45.4, 54.1, 55.9, 112.6, 125.1, 125.8, 126.7, 127.2, 128.1, 128.8, 128.8, 130.2, 130.6, 138.4, 139.2, 147.8, 155.1; HRMS (FAB): $m / z$ calcd for $\mathrm{C}_{22} \mathrm{H}_{25} \mathrm{ClN}_{3} \mathrm{OS}[\mathrm{M}+\mathrm{H}]^{+} 414.1407$; found: 414.1410 .

Compound 31x. Using the general procedure as described for 25a, compound 29x (33.2 $\mathrm{mg}, 0.080 \mathrm{mmol}$ ) was allowed to react for $3 \mathrm{~h}$. Purification by flash 
chromatography over aluminum oxide with $n$-hexane-EtOAc $(9: 1$ to $7: 3)$ gave the title compound $\mathbf{3 1 x}$ as colorless solid $\left(16.3 \mathrm{mg}, 57 \%\right.$ ): $\mathrm{mp} 175-178{ }^{\circ} \mathrm{C}$ (from $\mathrm{CHCl}_{3}-n$-hexane); IR (neat) $\mathrm{cm}^{-1}: 1620 \quad(\mathrm{C}=\mathrm{N}), 1568 \quad(\mathrm{C}=\mathrm{N}) ;{ }^{1} \mathrm{H}-\mathrm{NMR}$ $\left(400 \mathrm{MHz}_{\mathrm{CDCl}}\right) \delta: 1.96-2.02\left(\mathrm{~m}, 2 \mathrm{H}, \mathrm{CH}_{2}\right), 3.71\left(\mathrm{t}, J=5.6 \mathrm{~Hz}, 2 \mathrm{H}, \mathrm{CH}_{2}\right)$, $3.79\left(\mathrm{~s}, 3 \mathrm{H}, \mathrm{CH}_{3}\right), 4.04\left(\mathrm{t}, J=6.2 \mathrm{~Hz}, 2 \mathrm{H}, \mathrm{CH}_{2}\right), 6.89(\mathrm{~d}, J=8.5 \mathrm{~Hz}, 1 \mathrm{H}, \mathrm{Ar})$, $7.19(\mathrm{~d}, J=1.7 \mathrm{~Hz}, 1 \mathrm{H}, \mathrm{Ar}), 7.26-7.30$ (m, 2H, Ar), $7.36(\mathrm{dd}, J=8.3,1.7 \mathrm{~Hz}$, $1 \mathrm{H}, \mathrm{Ar}), 8.27$ (d, $J=8.3 \mathrm{~Hz}, 1 \mathrm{H}, \mathrm{Ar}) ;{ }^{13} \mathrm{C}-\mathrm{NMR}\left(125 \mathrm{MHz}, \mathrm{CDCl}_{3}\right) \delta: 20.9,43.8$, 44.8, 55.9, 112.5, 124.2, 125.3, 125.8, 127.5, 128.6, 128.9, 130.1 (2C), 130.1, 139.8, 146.6, 153.3, 155.0; HRMS (FAB): $m / z$ calcd for $\mathrm{C}_{18} \mathrm{H}_{17} \mathrm{ClN}_{3} \mathrm{OS}[\mathrm{M}+\mathrm{H}]^{+}$ 358.0781; found: 358.0783 .

\subsubsection{Synthesis of 3,4-Dihydro-9-(naphthalen-2-yl)-2H, 6H-pyrimido[1,2-c][1,3]benzothiazin-6-imine (32a)}

$N$-(tert-Butyl)-3,4-dihydro-9-(naphthalen-2-yl)-2H,6H-pyrimido[1,2-c] [1,3] benzothiazin-6-imine (30a). Using the general procedure as described for 22l, $\mathrm{N}$-(tert-butyl)-9-bromo-3,4-dihydro-2H,6H-pyrimido[1,2-c][1,3]benzothiazin6-imine 22k (52.8 $\mathrm{mg}, 0.15 \mathrm{mmol})$ was allowed to react with 2-naphthaleneboronic acid $(30.9 \mathrm{mg}, 0.18 \mathrm{mmol}$ ) for $1 \mathrm{~h}$. Purification by flash chromatography over aluminum oxide with $n$-hexane-EtOAc (1:0 to 9:1) gave the title compound 30a as colorless solid (56.3 mg, $94 \%$ ): $\mathrm{mp} 144-145{ }^{\circ} \mathrm{C}$ (from $n$-hexane); IR (neat) $\mathrm{cm}^{-1}$ : $1592(\mathrm{C}=\mathrm{N})$; ${ }^{1} \mathrm{H}-\mathrm{NMR}\left(500 \mathrm{MHz}, \mathrm{CDCl}_{3}\right) \delta: 1.42\left(\mathrm{~s}, 9 \mathrm{H}, 3 \times \mathrm{CH}_{3}\right)$, $1.91-1.96\left(\mathrm{~m}, 2 \mathrm{H}, \mathrm{CH}_{2}\right), 3.65\left(\mathrm{t}, J=5.2 \mathrm{~Hz}, 2 \mathrm{H}, \mathrm{CH}_{2}\right), 3.90(\mathrm{t}, J=5.7 \mathrm{~Hz}, 2 \mathrm{H}$, $\mathrm{CH}_{2}$ ), 7.45-7.51 (m, 3H, Ar), 7.55 (d, $\left.J=8.0 \mathrm{~Hz}, 1 \mathrm{H}, \mathrm{Ar}\right), 7.71(\mathrm{~d}, J=8.0 \mathrm{~Hz}$, 1H, Ar), 7.84-7.91 (m, 3H, Ar), 8.04 (s, 1H, Ar), 8.29 (d, J=8.6 Hz, 1H, Ar); ${ }^{13} \mathrm{C}-\mathrm{NMR}\left(125 \mathrm{MHz}, \mathrm{CDCl}_{3}\right) \delta: 21.9,30.0$ (3C), 45.1, 45.5, 54.2, 122.9, 125.0 (2C), 126.0, 126.3, 126.5, 126.6, 127.6, 128.3, 128.6, 129.0, 129.6, 132.9, 133.5, 136.7, 138.3, 142.8, 147.7; HRMS (FAB): $m / z$ calcd for $\mathrm{C}_{25} \mathrm{H}_{26} \mathrm{~N}_{3} \mathrm{~S}[\mathrm{M}+\mathrm{H}]^{+}$ 400.1847; found: 400.1848 .

Compound 32a. Using the general procedure as described for 25a, compound 30a $(45.3 \mathrm{mg}, 0.11 \mathrm{mmol}$ ) was allowed to react for $3 \mathrm{~h}$. Purification by flash chromatography over aluminum oxide with $n$-hexane-EtOAc $(9: 1$ to $7: 3)$ gave the title compound 32a as colorless solid $\left(28.2 \mathrm{mg}, 73 \%\right.$ ): $\mathrm{mp} 143-145{ }^{\circ} \mathrm{C}$ (from $\mathrm{CHCl}_{3}-n$-hexane); IR (neat) $\mathrm{cm}^{-1}: 1620 \quad(\mathrm{C}=\mathrm{N}), 1568 \quad(\mathrm{C}=\mathrm{N}) ;{ }^{1} \mathrm{H}-\mathrm{NMR}$ $\left(400 \mathrm{MHz} \mathrm{CDCl}_{3}\right) \delta: 1.96-2.02\left(\mathrm{~m}, 2 \mathrm{H}, \mathrm{CH}_{2}\right), 3.72\left(\mathrm{t}, J=5.6 \mathrm{~Hz}, 2 \mathrm{H}, \mathrm{CH}_{2}\right), 4.04$ (t, $\left.J=6.2 \mathrm{~Hz}, 2 \mathrm{H}, \mathrm{CH}_{2}\right), 7.36(\mathrm{~d}, J=1.8 \mathrm{~Hz}, 1 \mathrm{H}, \mathrm{Ar}), 7.49-7.52(\mathrm{~m}, 2 \mathrm{H}, \mathrm{Ar})$, 7.57 (dd, $J=8.5,1.8 \mathrm{~Hz}, 1 \mathrm{H}, \mathrm{Ar}), 7.68$ (dd, $J=8.5,1.8 \mathrm{~Hz}, 1 \mathrm{H}, \mathrm{Ar}), 7.84-7.91$ (m, 3H, Ar), 8.02 (d, $J=1.5 \mathrm{~Hz}, 1 \mathrm{H}, \mathrm{Ar}), 8.33(\mathrm{~d}, J=8.5 \mathrm{~Hz}, 1 \mathrm{H}, \mathrm{Ar}) ;{ }^{13} \mathrm{C}-\mathrm{NMR}$ $\left(100 \mathrm{MHz} \mathrm{CDCl}_{3}\right) \delta: 21.0,43.8,44.9,122.0,124.9,125.3$ (2C), 126.1, 126.4, 126.5, 127.6, 128.3, 128.7, 129.4 (2C), 133.0, 133.5, 136.3, 143.4, 146.5, 153.3; HRMS (FAB): $m / z$ calcd for $\mathrm{C}_{21} \mathrm{H}_{18} \mathrm{~N}_{3} \mathrm{~S}[\mathrm{M}+\mathrm{H}]^{+} 344.1221$; found: 344.1222 . 


\subsubsection{Synthesis of 3,4-Dihydro-9-(naphthalen-1-yl)-2H, 6H-pyrimido[1,2-c][1,3]benzothiazin-6-imine (32b)}

$N$-(tert-Butyl)-3,4-dihydro-9-(naphthalen-1-yl)-2H,6H-pyrimido[1,2-c][1,3] benzothiazin-6-imine (30b). Using the general procedure as described for 22l, $\mathrm{N}$-(tert-butyl)-9-bromo-3,4-dihydro-2H,6H-pyrimido[1,2-c][1,3]benzothiazin6-imine 22k $(52.8 \mathrm{mg}, 0.15 \mathrm{mmol})$ was allowed to react with 1-naphthaleneboronic acid $(30.9 \mathrm{mg}, 0.18 \mathrm{mmol})$ for $1 \mathrm{~h}$. Purification by flash chromatography over aluminum oxide with $n$-hexane-EtOAc (1:0 to 9:1) gave the title compound 30b as a colorless oil $(58.4 \mathrm{mg}, 97 \%)$ : IR (neat) $\mathrm{cm}^{-1}: 1590(\mathrm{C}=\mathrm{N})$; ${ }^{1} \mathrm{H}-\mathrm{NMR}$ $\left(400 \mathrm{MHz}, \mathrm{CDCl}_{3}\right) \delta: 1.40\left(\mathrm{~s}, 9 \mathrm{H}, 3 \times \mathrm{CH}_{3}\right), 1.92-1.98\left(\mathrm{~m}, 2 \mathrm{H}, \mathrm{CH}_{2}\right), 3.67(\mathrm{t}$, $\left.J=5.6 \mathrm{~Hz}, 2 \mathrm{H}, \mathrm{CH}_{2}\right), 3.92\left(\mathrm{t}, J=6.1 \mathrm{~Hz}, 2 \mathrm{H}, \mathrm{CH}_{2}\right), 7.25-7.25(\mathrm{~m}, 1 \mathrm{H}, \mathrm{Ar}), 7.34$ $(\mathrm{dd}, J=8.3,1.7 \mathrm{~Hz}, 1 \mathrm{H}, \mathrm{Ar}), 7.39-7.52(\mathrm{~m}, 4 \mathrm{H}, \mathrm{Ar}), 7.86(\mathrm{~d}, J=8.3 \mathrm{~Hz}, 2 \mathrm{H}$, Ar), $7.89(\mathrm{~d}, J=7.6 \mathrm{~Hz}, 1 \mathrm{H}, \mathrm{Ar}), 8.31(\mathrm{~d}, J=8.3 \mathrm{~Hz}, 1 \mathrm{H}, \mathrm{Ar}) ;{ }^{13} \mathrm{C}-\mathrm{NMR}$ $\left(100 \mathrm{MHz}, \mathrm{CDCl}_{3}\right) \delta: 22.0,30.0$ (3C), 45.1, 45.4, 54.2, 125.3, 125.6, 125.6, 125.9, $126.3,126.7,126.8,128.0,128.2$, 128.3, 128.3, 129.1, 131.2, 133.7, 138.2, 138.6, 142.8, 147.8; HRMS (FAB): $m / z$ calcd for $\mathrm{C}_{25} \mathrm{H}_{26} \mathrm{~N}_{3} \mathrm{~S}[\mathrm{M}+\mathrm{H}]^{+} 400.1847$; found: 400.1845 .

Compound 32b. Using the general procedure as described for 25a, compound 30b (46.4 mg, $0.12 \mathrm{mmol}$ ) was allowed to react for $3 \mathrm{~h}$. Purification by flash chromatography over aluminum oxide with $n$-hexane-EtOAc $(9: 1$ to $7: 3)$ gave the title compound 32b as colorless solid (34.4 mg, $86 \%$ ): $\mathrm{mp} 146-148{ }^{\circ} \mathrm{C}$ (from $\mathrm{CHCl}_{3}-n$-hexane); IR (neat) $\mathrm{cm}^{-1}: 1620 \quad(\mathrm{C}=\mathrm{N}), 1568 \quad(\mathrm{C}=\mathrm{N}) ;{ }^{1} \mathrm{H}-\mathrm{NMR}$ $\left(400 \mathrm{MHz}_{\mathrm{CDCl}}\right) \delta$ : $1.98-2.04\left(\mathrm{~m}, 2 \mathrm{H}, \mathrm{CH}_{2}\right), 3.73\left(\mathrm{t}, J=5.6 \mathrm{~Hz}, 2 \mathrm{H}, \mathrm{CH}_{2}\right)$, $4.06\left(\mathrm{t}, J=6.2 \mathrm{~Hz}, 2 \mathrm{H}, \mathrm{CH}_{2}\right), 7.17(\mathrm{~d}, J=1.7 \mathrm{~Hz}, 1 \mathrm{H}, \mathrm{Ar}), 7.35-7.52(\mathrm{~m}, 5 \mathrm{H}$, Ar), 7.81-7.91 (m, 3H, Ar), 8.33 (d, $J=8.3 \mathrm{~Hz}, 1 \mathrm{H}, \mathrm{Ar}) ;{ }^{13} \mathrm{C}-\mathrm{NMR}(100 \mathrm{MHz}$, $\left.\mathrm{CDCl}_{3}\right) \delta: 21.1,43.8,45.0,124.7,125.2,125.4,125.6,126.0,126.4,126.8,128.3$, 128.4 (2C), 128.7, 128.9, 131.1, 133.7, 138.2, 143.4, 146.5, 153.3; HRMS (FAB): $\mathrm{m} / \mathrm{z}$ calcd for $\mathrm{C}_{21} \mathrm{H}_{18} \mathrm{~N}_{3} \mathrm{~S}[\mathrm{M}+\mathrm{H}]^{+} 344.1221$; found: 344.1221 .

\subsubsection{Synthesis of 3,4-Dihydro-9-(3,4- methylenedioxyphenyl)-2H,6H-pyrimido[1,2-c] [1,3]benzothiazin-6-imine (32c)}

$\mathrm{N}$-(tert-Butyl)-3,4-dihydro-9-(3,4-methylenedioxyphenyl)-2H,6H-pyrimido [1, 2-c][1,3]benzothiazin-6-imine (30c). Using the general procedure as described for 22l, $\mathrm{N}$-(tert-butyl)-9-bromo-3,4-dihydro- $2 \mathrm{H}, 6 \mathrm{H}$-pyrimido[1,2-c][1,3]benzothiazin6-imine $22 \mathrm{k}(52.8 \mathrm{mg}, 0.15 \mathrm{mmol}$ ) was allowed to react with 3,4-(methylenedioxy)phenylboronic acid (29.9 $\mathrm{mg}, 0.18 \mathrm{mmol}$ ) for $1 \mathrm{~h}$. Purification by flash chromatography over aluminum oxide with $n$-hexane-EtOAc (1:0 to 9:1) gave the title compound 30c as colorless solid $\left(54.6 \mathrm{mg}, 93 \%\right.$ ): $\mathrm{mp} 173{ }^{\circ} \mathrm{C}$ (from $\mathrm{CHCl}_{3}$ $n$-hexane); IR (neat) $\mathrm{cm}^{-1}: 1591(\mathrm{C}=\mathrm{N}) ;{ }^{1} \mathrm{H}-\mathrm{NMR}\left(400 \mathrm{MHz}, \mathrm{CDCl}_{3}\right) \delta: 1.40(\mathrm{~s}$, 
9H, $\left.3 \times \mathrm{CH}_{3}\right), 1.89-1.95\left(\mathrm{~m}, 2 \mathrm{H}, \mathrm{CH}_{2}\right), 3.63\left(\mathrm{t}, J=5.5 \mathrm{~Hz}, 2 \mathrm{H}, \mathrm{CH}_{2}\right), 3.88(\mathrm{t}$, $\left.J=6.2 \mathrm{~Hz}, 2 \mathrm{H}, \mathrm{CH}_{2}\right), 5.99\left(\mathrm{~s}, 2 \mathrm{H}, \mathrm{CH}_{2}\right), 6.87$ (d, $\left.J=8.8 \mathrm{~Hz}, 1 \mathrm{H}, \mathrm{Ar}\right), 7.05-7.07$ (m, 2H, Ar), $7.24(\mathrm{~d}, J=2.0 \mathrm{~Hz}, 1 \mathrm{H}, \mathrm{Ar}), 7.34$ (dd, $J=8.3,2.0 \mathrm{~Hz}, 1 \mathrm{H}, \mathrm{Ar}), 8.21$ $(\mathrm{d}, J=8.3 \mathrm{~Hz}, 1 \mathrm{H}, \mathrm{Ar}) ;{ }^{13} \mathrm{C}-\mathrm{NMR}\left(100 \mathrm{MHz}, \mathrm{CDCl}_{3}\right) \delta: 21.9,30.0$ (3C), 45.1, 45.4, 54.2, 101.3, 107.4, 108.6, 120.7, 122.3, 124.5, 126.2, 128.9, 129.5, 133.7, 138.3, 142.6, 147.7, 147.7, 148.3; HRMS (FAB): $m / z$ calcd for $\mathrm{C}_{22} \mathrm{H}_{24} \mathrm{~N}_{3} \mathrm{O}_{2} \mathrm{~S}$ $[\mathrm{M}+\mathrm{H}]^{+}$394.1589; found: 394.1592 .

Compound 32c. Using the general procedure as described for 25a, compound 30c $(40.1 \mathrm{mg}, 0.102 \mathrm{mmol})$ was allowed to react for $2 \mathrm{~h}$. Purification by flash chromatography over aluminum oxide with $n$-hexane-EtOAc $(9: 1$ to 7:3) gave the title compound 32c as colorless solid (17.0 mg, 49\%): $\mathrm{mp} 169-170{ }^{\circ} \mathrm{C}$ (from $\mathrm{CHCl}_{3}-n$-hexane); IR (neat) $\mathrm{cm}^{-1}: 1619 \quad(\mathrm{C}=\mathrm{N}), 1568 \quad(\mathrm{C}=\mathrm{N}) ;{ }^{1} \mathrm{H}-\mathrm{NMR}$ $\left(400 \mathrm{MHz}, \mathrm{CDCl}_{3}\right) \delta: 1.95-2.01\left(\mathrm{~m}, 2 \mathrm{H}, \mathrm{CH}_{2}\right), 3.70\left(\mathrm{t}, J=5.5 \mathrm{~Hz}, 2 \mathrm{H}, \mathrm{CH}_{2}\right)$, $4.03\left(\mathrm{t}, J=6.1 \mathrm{~Hz}, 2 \mathrm{H}, \mathrm{CH}_{2}\right), 6.00\left(\mathrm{~s}, 2 \mathrm{H}, \mathrm{CH}_{2}\right), 6.87(\mathrm{~d}, J=7.8 \mathrm{~Hz}, 1 \mathrm{H}, \mathrm{Ar})$, 7.04-7.06 (m, 2H, Ar), 7.15 (d, $J=1.7 \mathrm{~Hz}, 1 \mathrm{H}, \mathrm{Ar}), 7.20$ (br s, 1H, NH), 7.36 (dd, $J=8.3,1.7 \mathrm{~Hz}, 1 \mathrm{H}, \mathrm{Ar}), 8.24(\mathrm{~d}, J=8.3 \mathrm{~Hz}, 1 \mathrm{H}, \mathrm{Ar}) ;{ }^{13} \mathrm{C}-\mathrm{NMR}(100 \mathrm{MHz}$, $\left.\mathrm{CDCl}_{3}\right) \delta: 21.0,43.8,45.0,101.3,107.4,108.7,120.8,121.3,124.8,125.1,129.3$, 129.3, 133.4, 143.1, 146.4, 147.9, 148.3, 153.4; HRMS (FAB): $\mathrm{m} / \mathrm{z}$ calcd for $\mathrm{C}_{18} \mathrm{H}_{16} \mathrm{~N}_{3} \mathrm{O}_{2} \mathrm{~S}[\mathrm{M}+\mathrm{H}]^{+}$338.0963; found: 338.0960 .

\subsubsection{Synthesis of 3,4-Dihydro-9-(2,3- dihydrobenzo[b][1,4]dioxin-6-yl)-2H,6H- pyrimido[1,2-c][1,3]benzothiazin-6-imine (32d)}

\section{$N$-(tert-Butyl)-3,4-dihydro-9-(2,3-dihydrobenzo[b][1,4]dioxin-6-yl)-2H,6H-py-} rimido[1,2-c][1,3]benzothiazin-6-imine $(30 \mathrm{~d})$. Using the general procedure as described for 22l, $N$-(tert-butyl)-9-bromo-3,4-dihydro-2H,6H-pyrimido[1,2c][1,3]benzothiazin-6-imine 22k $(52.8 \mathrm{mg}, 0.15 \mathrm{mmol})$ was allowed to react with (2,3-dihydrobenzo[b][1,4]dioxin-6-yl)boronic acid $(32.3 \mathrm{mg}, 0.18 \mathrm{mmol})$ for $1 \mathrm{~h}$. Purification by flash chromatography over aluminum oxide with $n$-hexane-EtOAc (1:0 to 9:1) gave the title compound 30d as a colorless oil $(63.9 \mathrm{mg}, 96 \%)$ : IR (neat) $\mathrm{cm}^{-1}: 1586(\mathrm{C}=\mathrm{N}) ;{ }^{1} \mathrm{H}-\mathrm{NMR}\left(500 \mathrm{MHz} \mathrm{CDCl}_{3}\right) \delta: 1.40\left(\mathrm{~s}, 9 \mathrm{H}, 3 \times \mathrm{CH}_{3}\right)$, 1.89-1.94 (m, 2H, $\left.\mathrm{CH}_{2}\right), 3.63\left(\mathrm{t}, J=5.4 \mathrm{~Hz}, 2 \mathrm{H}, \mathrm{CH}_{2}\right), 3.88(\mathrm{t}, J=6.0 \mathrm{~Hz}, 2 \mathrm{H}$, $\left.\mathrm{CH}_{2}\right), 4.28\left(\mathrm{~s}, 4 \mathrm{H}, 2 \times \mathrm{CH}_{2}\right), 6.92(\mathrm{~d}, J=8.6 \mathrm{~Hz}, 1 \mathrm{H}, \mathrm{Ar}), 7.07-7.09$ (m, 1H, Ar), $7.11(\mathrm{~d}, J=2.3 \mathrm{~Hz}, 1 \mathrm{H}, \mathrm{Ar}), 7.26$ (s, 1H, Ar), 7.36 (t, $J=4.0 \mathrm{~Hz}, 1 \mathrm{H}, \mathrm{Ar}), 8.21$ $(\mathrm{d}, J=8.6 \mathrm{~Hz}, 1 \mathrm{H}, \mathrm{Ar}) ;{ }^{13} \mathrm{C}-\mathrm{NMR}\left(125 \mathrm{MHz}, \mathrm{CDCl}_{3}\right) \delta: 21.9,30.0(3 \mathrm{C}), 45.1$, 45.4, 54.1, 64.4, 64.5, 115.8, 117.6, 120.0, 122.2, 124.4, 126.1, 128.9, 129.4, 132.9, 138.4, 142.3, 143.8 (2C), 147.7; HRMS (FAB): $m / z$ calcd for $\mathrm{C}_{23} \mathrm{H}_{26} \mathrm{~N}_{3} \mathrm{O}_{2} \mathrm{~S}$ $[\mathrm{M}+\mathrm{H}]^{+}$408.1746; found: 408.1746 .

Compound 32d. Using the general procedure as described for 25a, compound 30d $(45.1 \mathrm{mg}, 0.11 \mathrm{mmol})$ was allowed to react for $3 \mathrm{~h}$. Purification by flash chromatography over aluminum oxide with $n$-hexane-EtOAc $(9: 1$ to $7: 3)$ gave the 
title compound 32d as colorless solid (26.7 $\mathrm{mg}, 69 \%$ ): $\mathrm{mp} 174-176{ }^{\circ} \mathrm{C}$ (from $\mathrm{CHCl}_{3}-n$-hexane); IR (neat) $\mathrm{cm}^{-1}: 1619 \quad(\mathrm{C}=\mathrm{N}), 1567 \quad(\mathrm{C}=\mathrm{N}) ;{ }^{1} \mathrm{H}-\mathrm{NMR}$ $\left(500 \mathrm{MHz} \mathrm{CDCl}_{3}\right) \delta: 1.95-2.00\left(\mathrm{~m}, 2 \mathrm{H}, \mathrm{CH}_{2}\right), 3.70\left(\mathrm{t}, J=5.7 \mathrm{~Hz}, 2 \mathrm{H}, \mathrm{CH}_{2}\right), 4.03$ $\left(\mathrm{t}, J=6.3 \mathrm{~Hz}, 2 \mathrm{H}, \mathrm{CH}_{2}\right), 4.28\left(\mathrm{~s}, 4 \mathrm{H}, 2 \times \mathrm{CH}_{2}\right), 6.92(\mathrm{~d}, J=8.6 \mathrm{~Hz}, 1 \mathrm{H}, \mathrm{Ar})$, $7.06(\mathrm{dd}, J=8.6,2.3 \mathrm{~Hz}, 1 \mathrm{H}, \mathrm{Ar}), 7.09(\mathrm{~d}, J=2.3 \mathrm{~Hz}, 1 \mathrm{H}, \mathrm{Ar}), 7.17$ (d, $J=2.0 \mathrm{~Hz}, 1 \mathrm{H}, \mathrm{Ar}), 7.19$ (br s, $1 \mathrm{H}, \mathrm{NH}), 7.38$ (dd, $J=8.6,2.0 \mathrm{~Hz}, 1 \mathrm{H}, \mathrm{Ar}), 8.23$ $\left(\mathrm{d}, J=8.6 \mathrm{~Hz}, 1 \mathrm{H}, \mathrm{Ar} ;{ }^{13} \mathrm{C}-\mathrm{NMR}\left(125 \mathrm{MHz}, \mathrm{CDCl}_{3}\right) \delta: 21.0,43.8,44.9,64.3\right.$, $64.4,115.8,117.7,120.0,121.2,124.6,125.0,129.2,129.3,132.5,142.8,143.8$, 143.9, 146.4, 153.4; HRMS (FAB): $m / z$ calcd for $\mathrm{C}_{19} \mathrm{H}_{18} \mathrm{~N}_{3} \mathrm{O}_{2} \mathrm{~S}[\mathrm{M}+\mathrm{H}]^{+}$ 352.1120; found: 352.1121 .

\subsubsection{Synthesis of 3,4-Dihydro-9-(quinolin-6-yl)-2H, 6H-pyrimido[1,2-c][1,3]benzothiazin-6-imine (32e)}

$N$-(tert-Butyl)-3,4-dihydro-9-(quinolin-6-yl)-2H,6H-pyrimido[1,2-c][1,3]benzothiazin-6-imine (30e). Using the general procedure as described for $22 \mathbf{l}, \mathrm{N}$-(tertbutyl)-9-bromo-3,4-dihydro-2H,6H-pyrimido[1,2-c][1,3]benzothiazin-6-imine $22 \mathbf{k}$ $(52.8 \mathrm{mg}, 0.15 \mathrm{mmol})$ was allowed to react with 6-quinolineboronic acid $(31.1 \mathrm{mg}$, $0.18 \mathrm{mmol}$ ) for $1 \mathrm{~h}$. Purification by flash chromatography over aluminum oxide with $n$-hexane-EtOAc (9:1 to $1: 1)$ gave the title compound 30e as colorless solid (36.2 mg, $60 \%$ ): $\mathrm{mp} 179-180{ }^{\circ} \mathrm{C}$ (from $\mathrm{CHCl}_{3}-n$-hexane); IR (neat) $\mathrm{cm}^{-1}: 1590$ $(\mathrm{C}=\mathrm{N}) ;{ }^{1} \mathrm{H}-\mathrm{NMR}\left(400 \mathrm{MHz}, \mathrm{CDCl}_{3}\right) \delta: 1.42\left(\mathrm{~s}, 9 \mathrm{H}, 3 \times \mathrm{CH}_{3}\right), 1.91-1.97(\mathrm{~m}, 2 \mathrm{H}$, $\left.\mathrm{CH}_{2}\right), 3.66\left(\mathrm{t}, J=5.4 \mathrm{~Hz}, 2 \mathrm{H}, \mathrm{CH}_{2}\right), 3.91\left(\mathrm{t}, J=6.1 \mathrm{~Hz}, 2 \mathrm{H}, \mathrm{CH}_{2}\right), 7.42-7.46(\mathrm{~m}$, $2 \mathrm{H}, \mathrm{Ar}), 7.55$ (dd, $J=8.3,1.8 \mathrm{~Hz}, 1 \mathrm{H}, \mathrm{Ar}), 7.95$ (dd, $J=8.8,2.0 \mathrm{~Hz}, 1 \mathrm{H}, \mathrm{Ar}), 8.00$ (d, $J=1.5 \mathrm{~Hz}, 1 \mathrm{H}, \mathrm{Ar}), 8.16-8.21$ (m, 2H, Ar), 8.32 (d, $J=8.3 \mathrm{~Hz}, 1 \mathrm{H}, \mathrm{Ar}), 8.93$ $(\mathrm{dd}, J=4.1,1.5 \mathrm{~Hz}, 1 \mathrm{H}, \mathrm{Ar}) ;{ }^{13} \mathrm{C}-\mathrm{NMR}\left(100 \mathrm{MHz}, \mathrm{CDCl}_{3}\right) \delta: 21.9,30.0(3 \mathrm{C})$, 45.1, 45.4, 54.2, 121.6, 123.1, 125.0, 125.7, 126.9, 128.4, 128.7, 129.1, 129.8, 130.1, 136.2, 137.5, 138.0, 142.0, 147.6, 148.0, 150.7; HRMS (FAB): $\mathrm{m} / \mathrm{z}$ calcd for $\mathrm{C}_{24} \mathrm{H}_{25} \mathrm{~N}_{4} \mathrm{~S}[\mathrm{M}+\mathrm{H}]^{+} 401.1800$; found: 401.1802 .

Compound 32e. Using the general procedure as described for 25a, compound 30e $(27.0 \mathrm{mg}, 0.067 \mathrm{mmol})$ was allowed to react for $3 \mathrm{~h}$. Purification by flash chromatography over aluminum oxide with $n$-hexane-EtOAc $(9: 1$ to $1: 1)$ gave the title compound 32e as colorless solid (19.3 mg, $84 \%$ ): $\mathrm{mp} 165-167{ }^{\circ} \mathrm{C}$ (from $\mathrm{CHCl}_{3}-n$-hexane); IR (neat) $\mathrm{cm}^{-1}: 1620 \quad(\mathrm{C}=\mathrm{N}), 1572 \quad(\mathrm{C}=\mathrm{N}) ;{ }^{1} \mathrm{H}-\mathrm{NMR}$ $\left(400 \mathrm{MHz}, \mathrm{CDCl}_{3}\right) \delta$ : 1.98-2.04 (m, 2H, $\left.\mathrm{CH}_{2}\right), 3.73\left(\mathrm{t}, J=5.5 \mathrm{~Hz}, 2 \mathrm{H}, \mathrm{CH}_{2}\right)$, $4.06\left(\mathrm{t}, J=6.1 \mathrm{~Hz}, 2 \mathrm{H}, \mathrm{CH}_{2}\right), 7.38(\mathrm{~d}, J=1.7 \mathrm{~Hz}, 1 \mathrm{H}, \mathrm{Ar}), 7.45$ (dd, $J=8.0$, $4.3 \mathrm{~Hz}, 1 \mathrm{H}, \mathrm{Ar}), 7.58$ (dd, $J=8.3,1.7 \mathrm{~Hz}, 1 \mathrm{H}, \mathrm{Ar}), 7.94(\mathrm{dd}, J=8.8,2.2 \mathrm{~Hz}, 1 \mathrm{H}$, Ar), 8.01 (d, $J=2.2 \mathrm{~Hz}, 1 \mathrm{H}, \mathrm{Ar}), 8.18$ (d, $J=8.8 \mathrm{~Hz}, 1 \mathrm{H}, \mathrm{Ar}), 8.22$ (dd, $J=8.0$, $1.5 \mathrm{~Hz}, 1 \mathrm{H}, \mathrm{Ar}), 8.35$ (d, $J=8.3 \mathrm{~Hz}, 1 \mathrm{H}, \mathrm{Ar}), 8.94$ (dd, $J=4.3,1.5 \mathrm{~Hz}, 1 \mathrm{H}, \mathrm{Ar})$; ${ }^{13} \mathrm{C}-\mathrm{NMR}\left(100 \mathrm{MHz}, \mathrm{CDCl}_{3}\right) \delta: 21.0,43.8,45.0,121.7,122.1,125.3,125.8$, $125.9,128.3,128.6,129.5,129.6,130.2,136.3,137.2,142.5,146.3,148.0,150.8$, 
153.2; HRMS (FAB): $m / z$ calcd for $\mathrm{C}_{20} \mathrm{H}_{17} \mathrm{~N}_{4} \mathrm{~S}[\mathrm{M}+\mathrm{H}]^{+}$345.1174; found: 345.1175 .

\subsubsection{Synthesis of 3,4-Dihydro-9-[3- (trifluoromethylcarbonyl)indol-6-yl]-2H, 6H-pyrimido[1,2-c][1,3]benzothiazin-6-imine (32f)}

$N$-(tert-Butyl)-3,4-dihydro-9-(indol-6-yl)-2H,6H-pyrimido[1,2-c][1,3]benzothiazin-6-imine (30f). Using the general procedure as described for 221, $\mathrm{N}$-(tertbutyl)-9-bromo-3,4-dihydro-2 $H, 6 H$-pyrimido[1,2-c][1,3]benzothiazin-6-imine 22k $(52.8 \mathrm{mg}, 0.15 \mathrm{mmol})$ was allowed to react with indol-6-ylboronic acid $(29.0 \mathrm{mg}$, $0.18 \mathrm{mmol}$ ) for $1 \mathrm{~h}$. Purification by flash chromatography over aluminum oxide with $n$-hexane-EtOAc $(2: 1)$ gave the title compound $\mathbf{3 0 f}$ as colorless solid (56.0 mg, $96 \%$ ): $\mathrm{mp} 256{ }^{\circ} \mathrm{C}$ (from $\mathrm{MeOH}-\mathrm{CHCl}_{3}-n$-hexane); IR (neat) $\mathrm{cm}^{-1}$ : $1589(\mathrm{C}=\mathrm{N}) ;{ }^{1} \mathrm{H}-\mathrm{NMR}\left(500 \mathrm{MHz}, \mathrm{DMSO}-d_{6}\right) \delta: 1.39\left(\mathrm{~s}, 9 \mathrm{H}, 3 \times \mathrm{CH}_{3}\right), 1.82-1.87$ $\left(\mathrm{m}, 2 \mathrm{H}, \mathrm{CH}_{2}\right), 3.55\left(\mathrm{t}, J=5.4 \mathrm{~Hz}, 2 \mathrm{H}, \mathrm{CH}_{2}\right), 3.82\left(\mathrm{t}, J=6.0 \mathrm{~Hz}, 2 \mathrm{H}, \mathrm{CH}_{2}\right), 6.46$ (s, 1H, Ar), 7.37 (d, $J=8.3 \mathrm{~Hz}, 1 \mathrm{H}, \mathrm{Ar}), 7.42$ (t, $J=2.6 \mathrm{~Hz}, 1 \mathrm{H}, \mathrm{Ar}), 7.58-7.63$ (m, 3H, Ar), 7.72 (s, 1H, Ar), 8.21 (d, $J=8.3 \mathrm{~Hz}, 1 \mathrm{H}, \mathrm{Ar}), 11.21$ (br s, $1 \mathrm{H}, \mathrm{NH}$ ); ${ }^{13} \mathrm{C}-\mathrm{NMR}\left(125 \mathrm{MHz}, \mathrm{CDCl}_{3}-\mathrm{CD}_{3} \mathrm{OD}\right) \delta: 21.6,29.7$ (3C), 44.6, 45.4, 54.2, 109.6, $118.8,120.7$ (2C), 122.8, 125.1, 125.3, 125.5, 127.8, 128.5, 129.3, 132.7, 136.3, 138.4, 144.5, 149.3; HRMS (FAB): $m / z$ calcd for $\mathrm{C}_{23} \mathrm{H}_{25} \mathrm{~N}_{4} \mathrm{~S}[\mathrm{M}+\mathrm{H}]^{+} 389.1800$; found: 389.1800 .

Compound 32f. TFA (17 mL) was added to a mixture of $30 f(31.9 \mathrm{mg}$, $0.082 \mathrm{mmol})$ and MS4 $\AA$ (4.5 g, powder, activated by heating with Bunsen burner) in $\mathrm{CHCl}_{3}(3.0 \mathrm{~mL})$ and $\mathrm{MeOH}$ (5 drops). After being stirred under reflux for $8.5 \mathrm{~h}$, the mixture was concentrated. To a mixture of this residue in $\mathrm{CHCl}_{3}$ was added dropwise $\mathrm{Et}_{3} \mathrm{~N}$ at $0{ }^{\circ} \mathrm{C}$ to adjust $\mathrm{pH}$ to $8-9$. The whole was extracted with EtOAc. The extract was washed with sat. $\mathrm{NaHCO}_{3}$, brine, and dried over $\mathrm{MgSO}_{4}$. After concentration, the residue was purified by preparative TLC over aluminum oxide with $\mathrm{CHCl}_{3}-\mathrm{MeOH}(98: 2)$ to give the title compound $\mathbf{3 2 f}$ as pale yellow solid (17.8 mg, $51 \%$ ): $\mathrm{mp} 270{ }^{\circ} \mathrm{C}$ (decomp.) (from $\mathrm{MeOH}-\mathrm{CHCl}_{3}-n$-hexane); IR (neat) $\mathrm{cm}^{-1}: 1662(\mathrm{C}=\mathrm{O}), 1568(\mathrm{C}=\mathrm{N}) ;{ }^{1} \mathrm{H}-\mathrm{NMR}\left(500 \mathrm{MHz}, \mathrm{DMSO}-d_{6}\right) \delta: 1.86-$ $1.91\left(\mathrm{~m}, 2 \mathrm{H}, \mathrm{CH}_{2}\right), 3.61\left(\mathrm{t}, J=5.4 \mathrm{~Hz}, 2 \mathrm{H}, \mathrm{CH}_{2}\right), 3.94\left(\mathrm{t}, J=6.0 \mathrm{~Hz}, 2 \mathrm{H}, \mathrm{CH}_{2}\right)$, 7.61-7.62 (m, 2H, Ar), $7.71(\mathrm{dd}, J=8.6,1.7 \mathrm{~Hz}, 1 \mathrm{H}, \mathrm{Ar}), 7.88(\mathrm{~s}, 1 \mathrm{H}, \mathrm{Ar})$, 8.24-8.28 (m, 2H, Ar), 8.55 (d, $J=1.7 \mathrm{~Hz}, 1 \mathrm{H}, \mathrm{Ar}), 8.75$ (s, 1H, NH), 12.82 (br s, $1 \mathrm{H}, \mathrm{NH}) ;{ }^{13} \mathrm{C}-\mathrm{NMR}\left(100 \mathrm{MHz}, \mathrm{DMSO}-d_{6}\right) \delta: 20.7,43.1,44.4,108.8,111.1,116.8$ $(\mathrm{q}, J=291.3 \mathrm{~Hz}), 121.5,121.7,122.6,124.4,124.8,125.7,129.1,129.6,134.6$, $137.2,138.5(\mathrm{q}, J=4.7 \mathrm{~Hz}), 142.4,145.1,149.7,173.9(\mathrm{q}, J=33.9 \mathrm{~Hz})$; HRMS (FAB): $m / z$ calcd for $\mathrm{C}_{21} \mathrm{H}_{16} \mathrm{~F}_{3} \mathrm{~N}_{4} \mathrm{OS}[\mathrm{M}+\mathrm{H}]^{+}$429.0997; found: 429.1001 . 


\subsubsection{Synthesis of 3,4-Dihydro-9-[3- (trifluoromethylcarbonyl)indol-5-yl]-2H, 6H-pyrimido[1,2-c][1,3]benzothiazin-6-imine (32g)}

$N$-(tert-Butyl)-3,4-dihydro-9-(indol-5-yl)-2H,6H-pyrimido[1,2-c][1,3]benzothiazin-6-imine $\mathbf{( 3 0 g )}$. Using the general procedure as described for $\mathbf{2 2}$, $\mathrm{N}$-(tertbutyl)-9-bromo-3,4-dihydro-2H,6H-pyrimido[1,2-c][1,3]benzothiazin-6-imine 22k $(52.8 \mathrm{mg}, 0.15 \mathrm{mmol})$ was allowed to react with indol-5-ylboronic acid $(29.0 \mathrm{mg}$, $0.18 \mathrm{mmol}$ ) for $1 \mathrm{~h}$. Purification by flash chromatography over aluminum oxide with n-hexane-EtOAc $(2: 1)$ gave the title compound $\mathbf{3 0 g}$ as colorless solid (59.2 mg, > $99 \%$ ): mp $232-233{ }^{\circ} \mathrm{C}$ (from $\mathrm{MeOH}-\mathrm{CHCl}_{3}-n$-hexane); IR (neat) $\mathrm{cm}^{-1}: 1583(\mathrm{C}=\mathrm{N}) ;{ }^{1} \mathrm{H}-\mathrm{NMR} \quad\left(400 \mathrm{MHz}, \mathrm{CDCl}_{3}-\mathrm{CD}_{3} \mathrm{OD}\right) \delta: 1.41 \quad(\mathrm{~s}, 9 \mathrm{H}$, $\left.3 \times \mathrm{CH}_{3}\right), 1.91-1.97\left(\mathrm{~m}, 2 \mathrm{H}, \mathrm{CH}_{2}\right), 3.63\left(\mathrm{t}, J=5.4 \mathrm{~Hz}, 2 \mathrm{H}, \mathrm{CH}_{2}\right), 3.90(\mathrm{t}$, $\left.J=6.1 \mathrm{~Hz}, 2 \mathrm{H}, \mathrm{CH}_{2}\right), 6.58(\mathrm{~d}, J=3.0 \mathrm{~Hz}, 1 \mathrm{H}, \mathrm{Ar}), 7.23(\mathrm{~d}, J=3.0 \mathrm{~Hz}, 1 \mathrm{H}, \mathrm{Ar})$, 7.41-7.42 (m, 3H, Ar), 7.50 (dd, $J=8.5,0.9 \mathrm{~Hz}, 1 \mathrm{H}, \mathrm{Ar}), 7.86$ (s, 1H, Ar), 8.18 (d, $J=8.5 \mathrm{~Hz}, 1 \mathrm{H}, \mathrm{Ar}) ;{ }^{13} \mathrm{C}-\mathrm{NMR}\left(100 \mathrm{MHz}, \mathrm{CDCl}_{3}-\mathrm{CD}_{3} \mathrm{OD}\right) \delta: 21.8,29.9$ (3C), 44.9, 45.5, 54.2, 102.7, 111.4, 119.2, 121.2, 122.7, 125.1, 125.1, 125.3, 128.3, 128.6, 129.3, 131.1, 135.7, 138.6, 144.6, 148.8; HRMS (FAB): $m / z$ calcd for $\mathrm{C}_{23} \mathrm{H}_{25} \mathrm{~N}_{4} \mathrm{~S}$ $[\mathrm{M}+\mathrm{H}]^{+}$389.1800; found: 389.1800 .

Compound 32g. TFA $(17 \mathrm{~mL})$ was added to a mixture of $\mathbf{3 0 g}(28.5 \mathrm{mg}$, $0.073 \mathrm{mmol}$ ) and MS4 $\mathrm{A}$ (4.5 g, powder, activated by heating with Bunsen burner) in $\mathrm{CHCl}_{3}(3.0 \mathrm{~mL})$. After being stirred under reflux for $10 \mathrm{~h}$, the mixture was concentrated. To a mixture of this residue in $\mathrm{CHCl}_{3}$ was added dropwise $\mathrm{Et}_{3} \mathrm{~N}$ at $0{ }^{\circ} \mathrm{C}$ to adjust $\mathrm{pH}$ to $8-9$. The whole was extracted with EtOAc. The extract was washed with sat. $\mathrm{NaHCO}_{3}$, brine, and dried over $\mathrm{MgSO}_{4}$. After concentration, the residue was purified by preparative TLC over aluminum oxide with $\mathrm{CHCl}_{3}-\mathrm{MeOH}$ (98:2) to give the compound $\mathbf{3 2 \mathrm { g }}$ as pale yellow solid $(28.9 \mathrm{mg}, 92 \%)$ : $\mathrm{mp} 250{ }^{\circ} \mathrm{C}$ (decomp.) (from MeOH-CHCl $3-n$-hexane); IR (neat) $\mathrm{cm}^{-1}$ : $1659(\mathrm{C}=\mathrm{O}), 1613$ $(\mathrm{C}=\mathrm{N}), 1561(\mathrm{C}=\mathrm{N}) ;{ }^{1} \mathrm{H}-\mathrm{NMR}\left(500 \mathrm{MHz}, \mathrm{DMSO}-d_{6}\right) \delta: 1.86-1.91\left(\mathrm{~m}, 2 \mathrm{H}, \mathrm{CH}_{2}\right)$, $3.61\left(\mathrm{t}, J=5.4 \mathrm{~Hz}, 2 \mathrm{H}, \mathrm{CH}_{2}\right), 3.94\left(\mathrm{t}, J=5.7 \mathrm{~Hz}, 2 \mathrm{H}, \mathrm{CH}_{2}\right), 7.56-7.59(\mathrm{~m}, 2 \mathrm{H}$, Ar), 7.67-7.72 (m, 2H, Ar), 8.28 (d, J = 8.6 Hz, 1H, Ar), 8.45 (s, 1H, Ar), 8.56 (d, $J=1.7 \mathrm{~Hz}, 1 \mathrm{H}, \mathrm{Ar}), 8.75$ (br s, $1 \mathrm{H}, \mathrm{NH}), 12.82$ (br s, $1 \mathrm{H}, \mathrm{NH}) ;{ }^{13} \mathrm{C}-\mathrm{NMR}$ $\left(100 \mathrm{MHz}, \mathrm{DMSO}-d_{6}\right) \delta: 20.7,43.1,44.4,109.1,113.6,116.8(\mathrm{q}, J=290.5 \mathrm{~Hz})$, $119.2,121.6,123.7,124.6,124.6,126.4,129.1,129.6,133.9,136.6,138.4$ (q, $J=4.7 \mathrm{~Hz}), 143.0,145.2,149.7,173.9$ (q, $J=33.7 \mathrm{~Hz})$; HRMS (FAB): $m / z$ calcd for $\mathrm{C}_{21} \mathrm{H}_{16} \mathrm{~F}_{3} \mathrm{~N}_{4} \mathrm{OS}[\mathrm{M}+\mathrm{H}]^{+}$429.0997; found: 429.0991 .

\subsubsection{Synthesis of 3,4-Dihydro-9-(pyridin-3-yl)-2H, 6H-pyrimido[1,2-c][1,3]benzothiazin-6-imine (32h)}

$N$-(tert-Butyl)-3,4-dihydro-9-(pyridin-3-yl)-2H,6H-pyrimido[1,2-c][1,3]benzothiazin-6-imine (30h). Using the general procedure as described for $22 \mathrm{l}$, 
$N$-(tert-butyl)-9-bromo-3,4-dihydro-2H,6H-pyrimido[1,2-c][1,3]benzothiazin-6imine 22k (52.8 mg, $0.15 \mathrm{mmol}$ ) was allowed to react with 3-pyridineboronic acid (22.1 mg, $0.18 \mathrm{mmol}$ ) for $1 \mathrm{~h}$. Purification by flash chromatography over aluminum oxide with $n$-hexane-EtOAc (7:3) gave the title compound 30h as colorless solid (45.9 mg, $87 \%$ ): mp $143-144{ }^{\circ} \mathrm{C}$ (from $\mathrm{CHCl}_{3}-n$-hexane); IR (neat) $\mathrm{cm}^{-1}: 1595$ $(\mathrm{C}=\mathrm{N}) ;{ }^{1} \mathrm{H}-\mathrm{NMR}\left(400 \mathrm{MHz}, \mathrm{CDCl}_{3}\right) \delta: 1.40\left(\mathrm{~s}, 9 \mathrm{H}, 3 \times \mathrm{CH}_{3}\right), 1.91-1.96(\mathrm{~m}, 2 \mathrm{H}$, $\left.\mathrm{CH}_{2}\right), 3.65\left(\mathrm{t}, J=5.6 \mathrm{~Hz}, 2 \mathrm{H}, \mathrm{CH}_{2}\right), 3.89\left(\mathrm{t}, J=6.2 \mathrm{~Hz}, 2 \mathrm{H}, \mathrm{CH}_{2}\right), 7.33(\mathrm{~d}$, $J=1.7 \mathrm{~Hz}, 1 \mathrm{H}, \mathrm{Ar}), 7.36(\mathrm{dd}, J=7.8,4.9 \mathrm{~Hz}, 1 \mathrm{H}, \mathrm{Ar}), 7.41(\mathrm{dd}, J=8.5,1.7 \mathrm{~Hz}$, $1 \mathrm{H}, \mathrm{Ar}), 7.87$ (ddd, $J=7.8,2.2,1.5 \mathrm{~Hz}, 1 \mathrm{H}, \mathrm{Ar}), 8.30$ (d, $J=8.5 \mathrm{~Hz}, 1 \mathrm{H}, \mathrm{Ar}), 8.62$ $(\mathrm{dd}, J=4.9,1.5 \mathrm{~Hz}, 1 \mathrm{H}, \mathrm{Ar}), 8.85(\mathrm{~d}, J=2.2 \mathrm{~Hz}, 1 \mathrm{H}, \mathrm{Ar}) ;{ }^{13} \mathrm{C}-\mathrm{NMR}(100 \mathrm{MHz}$, $\left.\mathrm{CDCl}_{3}\right) \delta: 21.8,30.0$ (3C), 45.1, 45.4, 54.2, 122.8, 123.5, 124.7, 127.3, 129.3, 130.0, 134.2, 135.0, 137.8, 139.6, 147.5, 148.1, 149.2; HRMS (FAB): $m / z$ calcd for $\mathrm{C}_{20} \mathrm{H}_{23} \mathrm{~N}_{4} \mathrm{~S}[\mathrm{M}+\mathrm{H}]^{+}$351.1643; found: 351.1645.

Compound 32h. Using the general procedure as described for 25a, compound 30h $(36.3 \mathrm{mg}, 0.10 \mathrm{mmol})$ was allowed to react for $2 \mathrm{~h}$ with TFA $(1.0 \mathrm{~mL})$ and MS4A (150 mg). Purification by flash chromatography over aluminum oxide with $n$-hexane-EtOAc $(1: 1)$ gave the title compound $\mathbf{3 2 h}$ as colorless solid $(24.8 \mathrm{mg}$, $81 \%$ ): $\mathrm{mp} 191-193{ }^{\circ} \mathrm{C}$ (from $\mathrm{CHCl}_{3}-n$-hexane); IR (neat) $\mathrm{cm}^{-1}: 1616(\mathrm{C}=\mathrm{N})$, $1568(\mathrm{C}=\mathrm{N}) ;{ }^{1} \mathrm{H}-\mathrm{NMR}\left(400 \mathrm{MHz}, \mathrm{CDCl}_{3}\right) \delta: 1.97-2.03\left(\mathrm{~m}, 2 \mathrm{H}, \mathrm{CH}_{2}\right), 3.72(\mathrm{t}$, $\left.J=5.5 \mathrm{~Hz}, 2 \mathrm{H}, \mathrm{CH}_{2}\right), 4.05\left(\mathrm{t}, J=6.1 \mathrm{~Hz}, 2 \mathrm{H}, \mathrm{CH}_{2}\right), 7.24(\mathrm{~d}, J=1.7 \mathrm{~Hz}, 1 \mathrm{H}$, Ar), $7.38(\mathrm{dd}, J=7.9,4.8 \mathrm{~Hz}, 1 \mathrm{H}, \mathrm{Ar}), 7.43(\mathrm{dd}, J=8.3,1.7 \mathrm{~Hz}, 1 \mathrm{H}, \mathrm{Ar}), 7.86$ $(\mathrm{dt}, J=7.9,1.8 \mathrm{~Hz}, 1 \mathrm{H}, \mathrm{Ar}), 8.33(\mathrm{~d}, J=8.3 \mathrm{~Hz}, 1 \mathrm{H}, \mathrm{Ar}), 8.63$ (dd, $J=4.8$, $1.8 \mathrm{~Hz}, 1 \mathrm{H}, \mathrm{Ar}), 8.83(\mathrm{~d}, J=1.8 \mathrm{~Hz}, 1 \mathrm{H}, \mathrm{Ar}) ;{ }^{13} \mathrm{C}-\mathrm{NMR}\left(100 \mathrm{MHz}, \mathrm{CDCl}_{3}\right) \delta$ : 21.0, 43.8, 44.9, 121.8, 123.6, 124.9, 126.2, 129.7, 129.8, 134.2, 134.7, 140.1, 146.2, 148.1, 149.3, 152.9; HRMS (FAB): $m / z$ calcd for $\mathrm{C}_{16} \mathrm{H}_{15} \mathrm{~N}_{4} \mathrm{~S}[\mathrm{M}+\mathrm{H}]^{+}$ 295.1017; found: 295.1013 .

\subsubsection{Synthesis of 3,4-Dihydro-9-(pyridin-4-yl)-2H, 6H-pyrimido[1,2-c][1,3]benzothiazin-6-imine (32i)}

\section{$N$-(tert-Butyl)-3,4-dihydro-9-(pyridin-4-yl)-2H,6 $H$-pyrimido[1,2-c $][1,3]$ benzo-}

thiazin-6-imine (30i). Using the general procedure as described for $221, \mathrm{~N}$-(tertbutyl)-9-bromo-3,4-dihydro-2H,6H-pyrimido[1,2-c][1,3]benzothiazin-6-imine $22 k$ (52.8 $\mathrm{mg}, 0.15 \mathrm{mmol})$ was allowed to react with 4-pyridineboronic acid $(22.1 \mathrm{mg}$, $0.18 \mathrm{mmol}$ ) for $1 \mathrm{~h}$. Purification by flash chromatography over aluminum oxide with $n$-hexane-EtOAc (7:3) gave the title compound 30i as colorless solid (27.6 $\mathrm{mg}$, $52 \%$ ): mp 196-197 ${ }^{\circ} \mathrm{C}$ (from $\mathrm{CHCl}_{3}-n$-hexane); IR (neat) $\mathrm{cm}^{-1}: 1593(\mathrm{C}=\mathrm{N}) ;{ }^{1} \mathrm{H}$ NMR $\left(500 \mathrm{MHz}, \mathrm{CDCl}_{3}\right) \delta: 1.41\left(\mathrm{~s}, 9 \mathrm{H}, 3 \times \mathrm{CH}_{3}\right), 1.91-1.96\left(\mathrm{~m}, 2 \mathrm{H}, \mathrm{CH}_{2}\right), 3.65$ (t, $\left.J=5.4 \mathrm{~Hz}, 2 \mathrm{H}, \mathrm{CH}_{2}\right), 3.89\left(\mathrm{t}, J=6.0 \mathrm{~Hz}, 2 \mathrm{H}, \mathrm{CH}_{2}\right), 7.38(\mathrm{~s}, 1 \mathrm{H}, \mathrm{Ar}), 7.45$ (d, $J=8.6 \mathrm{~Hz}, 1 \mathrm{H}, \mathrm{Ar}), 7.49$ (d, $J=5.0 \mathrm{~Hz}, 2 \mathrm{H}, \mathrm{Ar}), 8.30(\mathrm{~d}, J=8.6 \mathrm{~Hz}, 1 \mathrm{H}, \mathrm{Ar})$, $8.67(\mathrm{~d}, J=5.0 \mathrm{~Hz}, 2 \mathrm{H}, \mathrm{Ar}) ;{ }^{13} \mathrm{C}-\mathrm{NMR}\left(100 \mathrm{MHz}, \mathrm{CDCl}_{3}\right) \delta: 21.8,30.0(3 \mathrm{C}), 45.1$, 45.4, 54.2, 121.4 (2C), 122.8, 124.4, 128.1, 129.3, 130.1, 137.6, 139.8, 146.6, 147.4, 
150.4 (2C); HRMS (FAB): $m / z$ calcd for $\mathrm{C}_{20} \mathrm{H}_{23} \mathrm{~N}_{4} \mathrm{~S}[\mathrm{M}+\mathrm{H}]^{+}$351.1643; found: 351.1644 .

Compound 32i. Using the general procedure as described for 25a, compound 30i $(23.4 \mathrm{mg}, 0.067 \mathrm{mmol}$ ) was allowed to react for $2 \mathrm{~h}$. Purification by flash chromatography over aluminum oxide with $n$-hexane-EtOAc $(1: 1$ to $0: 1)$ gave the title compound $\mathbf{3 2 i}$ as colorless solid $\left(15.1 \mathrm{mg}, 77 \%\right.$ ): $\mathrm{mp} 154-155^{\circ} \mathrm{C}$ (from $\mathrm{CHCl}_{3}-n$-hexane); IR (neat) $\mathrm{cm}^{-1}: 1620 \quad(\mathrm{C}=\mathrm{N}), 1575 \quad(\mathrm{C}=\mathrm{N}) ;{ }^{1} \mathrm{H}-\mathrm{NMR}$ $\left(500 \mathrm{MHz}, \mathrm{CDCl}_{3}\right) \delta: 1.97-2.02\left(\mathrm{~m}, 2 \mathrm{H}, \mathrm{CH}_{2}\right), 3.72\left(\mathrm{t}, J=5.4 \mathrm{~Hz}, 2 \mathrm{H}, \mathrm{CH}_{2}\right)$, $4.04\left(\mathrm{t}, J=6.3 \mathrm{~Hz}, 2 \mathrm{H}, \mathrm{CH}_{2}\right), 7.29$ (s, 1H, Ar), 7.46-7.48 (m, 3H, Ar), 8.33 (d, $J=8.6 \mathrm{~Hz}, 1 \mathrm{H}, \mathrm{Ar}), 8.68(\mathrm{~d}, J=4.6 \mathrm{~Hz}, 2 \mathrm{H}, \mathrm{Ar}) ;{ }^{13} \mathrm{C}-\mathrm{NMR}\left(100 \mathrm{MHz}, \mathrm{CDCl}_{3}\right)$ $\delta: 21.0,43.8,45.0,121.4$ (2C), 121.9, 124.8, 127.1, 129.7, 129.9, 140.3, 146.1, 146.3, 150.4 (2C), 152.8; Anal. calcd for $\mathrm{C}_{16} \mathrm{H}_{14} \mathrm{~N}_{4} \mathrm{~S}$ : C, 65.28; H, 4.79; N, 19.03. Found: C, 65.35; H, 4.63; N, 19.24.

\subsubsection{Synthesis of 9-(Furan-2-yl)-3,4-dihydro-2H, 6H-pyrimido[1,2-c][1,3]benzothiazin-6-imine (32j)}

$N$-(tert-Butyl)-9-(furan-2-yl)-3,4-dihydro-2H,6H-pyrimido[1,2-c][1,3]benzothiazin-6-imine $(\mathbf{3 0 j})$. Using the general procedure as described for $221, N$ (tert-butyl)-9-bromo-3,4-dihydro-2H,6H-pyrimido[1,2-c][1,3]benzothiazin6-imine 22k (52.8 mg, $0.15 \mathrm{mmol})$ was allowed to react with 2-furanboronic acid (20.1 mg, $0.18 \mathrm{mmol}$ ) for $1 \mathrm{~h}$. Purification by flash chromatography over aluminum oxide with $n$-hexane-EtOAc (1:0 to 9:1) gave the title compound $\mathbf{3 0 j}$ as colorless solid (42.4 mg, $83 \%$ ): $\mathrm{mp} 128-130{ }^{\circ} \mathrm{C}$ (from $n$-hexane); IR (neat) $\mathrm{cm}^{-1}$ : $1596(\mathrm{C}=\mathrm{N}) ;{ }^{1} \mathrm{H}-\mathrm{NMR}\left(400 \mathrm{MHz}, \mathrm{CDCl}_{3}\right) \delta: 1.40\left(\mathrm{~s}, 9 \mathrm{H}, 3 \times \mathrm{CH}_{3}\right), 1.89-1.94$ $\left(\mathrm{m}, 2 \mathrm{H}, \mathrm{CH}_{2}\right), 3.63\left(\mathrm{t}, J=5.5 \mathrm{~Hz}, 2 \mathrm{H}, \mathrm{CH}_{2}\right), 3.87\left(\mathrm{t}, J=6.1 \mathrm{~Hz}, 2 \mathrm{H}, \mathrm{CH}_{2}\right)$, 6.47-6.49 (m, 1H, Ar), 6.71 (d, $J=3.4 \mathrm{~Hz}, 1 \mathrm{H}, \mathrm{Ar}), 7.42-7.48$ (m, 3H, Ar), 8.20 $(\mathrm{d}, J=8.3 \mathrm{~Hz}, 1 \mathrm{H}, \mathrm{Ar}) ;{ }^{13} \mathrm{C}-\mathrm{NMR}\left(100 \mathrm{MHz}, \mathrm{CDCl}_{3}\right) \delta: 21.9,30.0(3 \mathrm{C}), 45.1$, 45.4, 54.2, 106.7, 111.9, 119.2, 121.4, 126.3, 128.8, 129.6, 132.3, 138.2, 142.8, 147.6, 152.5.; HRMS (FAB): $m / z$ calcd for $\mathrm{C}_{19} \mathrm{H}_{22} \mathrm{~N}_{3} \mathrm{OS}[\mathrm{M}+\mathrm{H}]^{+} 340.1484$; found: 340.1484 .

Compound 32j. Using the general procedure as described for $\mathbf{2 5 a}$, compound 30j (30.3 mg, $0.089 \mathrm{mmol})$ was allowed to react for $4 \mathrm{~h}$ with TFA $(1.0 \mathrm{~mL})$ and MS4A (150 mg). Purification by flash chromatography over aluminum oxide with $n$-hexane-EtOAc (7:3) gave the title compound $\mathbf{3 2} \mathbf{j}$ as colorless solid (18.3 $\mathrm{mg}$, $73 \%$ ): $\mathrm{mp} 133{ }^{\circ} \mathrm{C}$ (from $\mathrm{CHCl}_{3}-n$-hexane); IR (neat) $\mathrm{cm}^{-1}: 1620(\mathrm{C}=\mathrm{N}), 1567$ $(\mathrm{C}=\mathrm{N})$; ${ }^{1} \mathrm{H}-\mathrm{NMR}\left(400 \mathrm{MHz}, \mathrm{CDCl}_{3}\right) \delta: 1.95-2.01\left(\mathrm{~m}, 2 \mathrm{H}, \mathrm{CH}_{2}\right), 3.70(\mathrm{t}$, $\left.J=5.6 \mathrm{~Hz}, 2 \mathrm{H}, \mathrm{CH}_{2}\right), 4.02\left(\mathrm{t}, J=6.1 \mathrm{~Hz}, 2 \mathrm{H}, \mathrm{CH}_{2}\right), 6.49(\mathrm{dd}, J=3.4,1.7 \mathrm{~Hz}$, $1 \mathrm{H}, \mathrm{Ar}), 6.73(\mathrm{~d}, J=3.4 \mathrm{~Hz}, 1 \mathrm{H}, \mathrm{Ar}), 7.21$ (br s, $1 \mathrm{H}, \mathrm{NH}), 7.33$ (d, $J=1.7 \mathrm{~Hz}$, $1 \mathrm{H}, \mathrm{Ar}), 7.47-7.49(\mathrm{~m}, 2 \mathrm{H}, \mathrm{Ar}), 8.22(\mathrm{~d}, J=8.5 \mathrm{~Hz}, 1 \mathrm{H}, \mathrm{Ar}) ;{ }^{13} \mathrm{C}-\mathrm{NMR}$ $\left(100 \mathrm{MHz}, \mathrm{CDCl}_{3}\right) \delta: 21.0,43.8,45.0,107.1,112.0,118.2,121.6,125.2,129.3$, 129.5, 132.7, 143.0, 146.3, 152.2, 153.3; Anal. calcd for $\mathrm{C}_{15} \mathrm{H}_{13} \mathrm{~N}_{3} \mathrm{OS}: \mathrm{C}, 63.58 ; \mathrm{H}$, 4.62; N, 14.83. Found: C, 63.40; H, 4.46; N, 14.72. 


\subsubsection{Synthesis of 9-(Benzofuran-2-yl)-3,4-dihydro-2H, 6H-pyrimido[1,2-c][1,3]benzothiazin-6-imine (32k)}

9-(Benzofuran-2-yl)- $N$-(tert-butyl)-3,4-dihydro-2H,6H-pyrimido[1,2-c][1,3] benzothiazin-6-imine (30k) Using the general procedure as described for 22l, $\mathrm{N}$-(tertbutyl)-9-bromo-3,4-dihydro-2H,6H-pyrimido[1,2-c][1,3]benzothiazin-6-imine 22k (52.8 $\mathrm{mg}, 0.15 \mathrm{mmol}$ ) was allowed to react with 2-benzofuranboronic acid (29.2 mg, $0.18 \mathrm{mmol}$ ) for $1 \mathrm{~h}$. Purification by flash chromatography over aluminum oxide with $n$-hexane-EtOAc (1:0 to 9:1) gave the title compound 30k as colorless solid (54.3 mg, $93 \%$ ): $\mathrm{mp} 211{ }^{\circ} \mathrm{C}$ (from $\mathrm{CHCl}_{3}-n$-hexane); IR (neat) $\mathrm{cm}^{-1}: 1595$ $(\mathrm{C}=\mathrm{N}) ;{ }^{1} \mathrm{H}-\mathrm{NMR}\left(500 \mathrm{MHz}, \mathrm{CDCl}_{3}\right) \delta: 1.41\left(\mathrm{~s}, 9 \mathrm{H}, 3 \times \mathrm{CH}_{3}\right), 1.90-1.94(\mathrm{~m}, 2 \mathrm{H}$, $\left.\mathrm{CH}_{2}\right), 3.64\left(\mathrm{t}, J=5.4 \mathrm{~Hz}, 2 \mathrm{H}, \mathrm{CH}_{2}\right), 3.88\left(\mathrm{t}, J=6.0 \mathrm{~Hz}, 2 \mathrm{H}, \mathrm{CH}_{2}\right), 7.06(\mathrm{~s}, 1 \mathrm{H}$, Ar), $7.23(\mathrm{t}, J=7.4 \mathrm{~Hz}, 1 \mathrm{H}, \mathrm{Ar}), 7.30(\mathrm{t}, J=7.4 \mathrm{~Hz}, 1 \mathrm{H}, \mathrm{Ar}), 7.50$ (d, $J=7.4 \mathrm{~Hz}$, $1 \mathrm{H}, \mathrm{Ar}), 7.58$ (d, $J=7.4 \mathrm{~Hz}, 1 \mathrm{H}, \mathrm{Ar}), 7.62-7.64$ (m, 2H, Ar), 8.25 (d, $J=8.0 \mathrm{~Hz}$, $1 \mathrm{H}, \mathrm{Ar}) ;{ }^{13} \mathrm{C}-\mathrm{NMR}\left(125 \mathrm{MHz}, \mathrm{CDCl}_{3}\right) \delta: 21.9,30.0$ (3C), 45.2, 45.4, 54.2, 103.0, 111.2, 120.4, 121.2, 122.3, 123.1, 124.9, 127.3, 128.9 (2C), 129.8, 132.0, 138.0, 147.5, 154.2, 155.0; HRMS (FAB): $m / z$ calcd for $\mathrm{C}_{23} \mathrm{H}_{24} \mathrm{~N}_{3} \mathrm{OS}[\mathrm{M}+\mathrm{H}]^{+}$390.1640; found: 390.1645 .

Compound 32k. Using the general procedure as described for 25a, compound 30k $(41.5 \mathrm{mg}, 0.11 \mathrm{mmol})$ was allowed to react for $3 \mathrm{~h}$. Purification by flash chromatography over aluminum oxide with $n$-hexane-EtOAc $(9: 1$ to $7: 3)$ gave the title compound 32k as pale yellow solid (30.5 mg, $85 \%$ ): $\mathrm{mp} 189-191{ }^{\circ} \mathrm{C}$ (from $\mathrm{CHCl}_{3}-n$-hexane); IR (neat) $\mathrm{cm}^{-1}: 1620(\mathrm{C}=\mathrm{N}), 1565 \quad(\mathrm{C}=\mathrm{N}) ;{ }^{1} \mathrm{H}-\mathrm{NMR}$ $\left(500 \mathrm{MHz} \mathrm{CDCl}_{3}\right) \delta: 1.95-2.00\left(\mathrm{~m}, 2 \mathrm{H}, \mathrm{CH}_{2}\right), 3.70\left(\mathrm{t}, J=5.4 \mathrm{~Hz}, 2 \mathrm{H}, \mathrm{CH}_{2}\right)$, $4.02\left(\mathrm{t}, J=6.3 \mathrm{~Hz}, 2 \mathrm{H}, \mathrm{CH}_{2}\right), 7.07(\mathrm{~s}, 1 \mathrm{H}, \mathrm{Ar}), 7.22-7.25(\mathrm{~m}, 1 \mathrm{H}, \mathrm{Ar}), 7.31(\mathrm{t}$, $J=7.2 \mathrm{~Hz}, 1 \mathrm{H}, \mathrm{Ar}), 7.50-7.51(\mathrm{~m}, 2 \mathrm{H}, \mathrm{Ar}), 7.58$ (d, $J=7.2 \mathrm{~Hz}, 1 \mathrm{H}, \mathrm{Ar}), 7.65$ $(\mathrm{dd}, J=8.6,1.1 \mathrm{~Hz}, 1 \mathrm{H}, \mathrm{Ar}), 8.28(\mathrm{~d}, J=8.6 \mathrm{~Hz}, 1 \mathrm{H}, \mathrm{Ar}) ;{ }^{13} \mathrm{C}-\mathrm{NMR}(125 \mathrm{MHz}$, $\left.\mathrm{CDCl}_{3}\right) \delta: 21.0,43.8,45.0,103.4,111.3,119.4,121.2,122.6,123.2,125.1,126.2$, 128.8, 129.3, 129.6, 132.4, 146.2, 153.1, 153.9, 155.1; HRMS (FAB): $m / z$ calcd for $\mathrm{C}_{19} \mathrm{H}_{16} \mathrm{~N}_{3} \mathrm{OS}[\mathrm{M}+\mathrm{H}]^{+}$334.1014; found: 334.1017.

\subsubsection{Synthesis of 3,4-Dihydro-9-(thiophen-3-yl)-2H, 6H-pyrimido[1,2-c][1,3]benzothiazin-6-imine (32l)}

$N$-(tert-Butyl)-3,4-dihydro-9-(thiophen-3-yl)-2H,6H-pyrimido[1,2-c][1,3]benzothiazin-6-imine (301). Using the general procedure as described for 22l, $\mathrm{N}$-(tertbutyl)-9-bromo-3,4-dihydro-2H,6H-pyrimido[1,2-c][1,3]benzothiazin-6-imine 22k (52.8 $\mathrm{mg}, 0.15 \mathrm{mmol}$ ) was allowed to react with 3-thiopheneboronic acid (23.0 $\mathrm{mg}$, $0.18 \mathrm{mmol}$ ) for $1 \mathrm{~h}$. Purification by flash chromatography over aluminum oxide with $n$-hexane-EtOAc (1:0 to 9:1) gave the title compound $\mathbf{3 0 1}$ as colorless solid (54.0 mg, >99\%): mp 132-133 ${ }^{\circ} \mathrm{C}$ (from $n$-hexane); IR (neat) $\mathrm{cm}^{-1}: 1592(\mathrm{C}=\mathrm{N}$ ); ${ }^{1} \mathrm{H}-\mathrm{NMR}\left(400 \mathrm{MHz}, \mathrm{CDCl}_{3}\right) \delta: 1.40\left(\mathrm{~s}, 9 \mathrm{H}, 3 \times \mathrm{CH}_{3}\right), 1.89-1.95\left(\mathrm{~m}, 2 \mathrm{H}, \mathrm{CH}_{2}\right)$, 
$3.63\left(\mathrm{t}, J=5.6 \mathrm{~Hz}, 2 \mathrm{H}, \mathrm{CH}_{2}\right), 3.88\left(\mathrm{t}, J=6.2 \mathrm{~Hz}, 2 \mathrm{H}, \mathrm{CH}_{2}\right), 7.32(\mathrm{~d}, J=2.0 \mathrm{~Hz}$, $1 \mathrm{H}, \mathrm{Ar}), 7.36-7.43(\mathrm{~m}, 3 \mathrm{H}, \mathrm{Ar}), 7.50(\mathrm{dd}, J=2.7,1.5 \mathrm{~Hz}, 1 \mathrm{H}, \mathrm{Ar}), 8.21(\mathrm{~d}$, $J=8.5 \mathrm{~Hz}, 1 \mathrm{H}, \mathrm{Ar}) ;{ }^{13} \mathrm{C}-\mathrm{NMR}\left(100 \mathrm{MHz}, \mathrm{CDCl}_{3}\right) \delta: 21.9,30.0$ (3C), 45.1, 45.4, 54.2, 121.5, 121.9, 124.1, 126.0, 126.3, 126.6, 129.0, 129.6, 137.4, 138.2, 140.6, 147.7; HRMS (FAB): $m / z$ calcd for $\mathrm{C}_{19} \mathrm{H}_{22} \mathrm{~N}_{3} \mathrm{~S}_{2}[\mathrm{M}+\mathrm{H}]^{+} 356.1255$; found: 356.1253 .

Compound 32l. Using the general procedure as described for 25a, compound 301 ( $37.6 \mathrm{mg}, 0.11 \mathrm{mmol})$ was allowed to react for $2 \mathrm{~h}$ with TFA $(1.0 \mathrm{~mL})$ and MS4A (150 mg). Purification by flash chromatography over aluminum oxide with $n$-hexane-EtOAc (4:1) gave the title compound $32 \mathrm{l}$ as colorless solid $(25.9 \mathrm{mg}$, $82 \%$ ): mp $120-121{ }^{\circ} \mathrm{C}$ (from $\mathrm{CHCl}_{3}-n$-hexane); IR (neat) $\mathrm{cm}^{-1}: 1619(\mathrm{C}=\mathrm{N})$, $1569(\mathrm{C}=\mathrm{N}) ;{ }^{1} \mathrm{H}-\mathrm{NMR}\left(400 \mathrm{MHz}, \mathrm{CDCl}_{3}\right) \delta: 1.96-2.01\left(\mathrm{~m}, 2 \mathrm{H}, \mathrm{CH}_{2}\right), 3.70(\mathrm{t}$, $\left.J=5.4 \mathrm{~Hz}, 2 \mathrm{H}, \mathrm{CH}_{2}\right), 4.03\left(\mathrm{t}, J=6.1 \mathrm{~Hz}, 2 \mathrm{H}, \mathrm{CH}_{2}\right), 7.23(\mathrm{~d}, J=1.1 \mathrm{~Hz}, 1 \mathrm{H}$, Ar), 7.35-7.41 (m, 2H, Ar), 7.44 (dd, $J=8.3,1.1 \mathrm{~Hz}, 1 \mathrm{H}, \mathrm{Ar}), 7.51-7.52(\mathrm{~m}, 1 \mathrm{H}$, Ar), $8.24(\mathrm{~d}, J=8.3 \mathrm{~Hz}, 1 \mathrm{H}, \mathrm{Ar}) ;{ }^{13} \mathrm{C}-\mathrm{NMR}\left(100 \mathrm{MHz}, \mathrm{CDCl}_{3}\right) \delta: 21.0,43.8$, 44.9, 120.9, 121.8, 124.4, 125.0, 125.9, 126.7, 129.4 (2C), 138.0, 140.3, 146.5, 153.3; Anal. calcd for $\mathrm{C}_{15} \mathrm{H}_{13} \mathrm{~N}_{3} \mathrm{~S}_{2}$ : C, 60.17; H, 4.38; N, 14.03. Found: C, 60.12; $\mathrm{H}, 4.11 ; \mathrm{N}, 14.04$.

\subsubsection{Synthesis of 9-(Benzothiophen-2-yl)-3,4-dihydro- 2H,6H-pyrimido[1,2-c][1,3]benzothiazin-6-imine (32m)}

9-(Benzothiophen-2-yl)- $N$-(tert-butyl)-3,4-dihydro-2H,6H-pyrimido[1,2-c] [1,3] benzothiazin-6-imine (30m). Using the general procedure as described for $22 \mathrm{l}, \mathrm{N}$ (tert-butyl)-9-bromo-3,4-dihydro-2H,6 $\mathrm{H}$-pyrimido[1,2-c][1,3]benzothiazin-6-imine 22k (52.8 mg, $0.15 \mathrm{mmol})$ was allowed to react with 2-benzothiopheneboronic acid $(32.0 \mathrm{mg}, 0.18 \mathrm{mmol})$ for $1 \mathrm{~h}$. Purification by flash chromatography over aluminum oxide with $n$-hexane-EtOAc (1:0 to 9:1) gave the title compound 30m as colorless solid (44.8 mg, $74 \%$ ): $\mathrm{mp} 222-223{ }^{\circ} \mathrm{C}$ (from $\mathrm{CHCl}_{3}-n$-hexane); IR (neat) $\mathrm{cm}^{-1}: 1590(\mathrm{C}=\mathrm{N}) ;{ }^{1} \mathrm{H}-\mathrm{NMR}\left(500 \mathrm{MHz}, \mathrm{CDCl}_{3}\right) \delta: 1.41\left(\mathrm{~s}, 9 \mathrm{H}, 3 \times \mathrm{CH}_{3}\right)$, 1.90-1.94 (m, 2H, $\left.\mathrm{CH}_{2}\right), 3.63\left(\mathrm{t}, J=5.4 \mathrm{~Hz}, 2 \mathrm{H}, \mathrm{CH}_{2}\right), 3.88(\mathrm{t}, J=6.3 \mathrm{~Hz}, 2 \mathrm{H}$, $\mathrm{CH}_{2}$ ), 7.30-7.36 (m, 2H, Ar), 7.42 (d, $\left.J=1.7 \mathrm{~Hz}, 1 \mathrm{H}, \mathrm{Ar}\right), 7.52$ (dd, $J=8.6$, $1.7 \mathrm{~Hz}, 1 \mathrm{H}, \mathrm{Ar}), 7.58$ (s, 1H, Ar), $7.76(\mathrm{dd}, J=7.2,1.4 \mathrm{~Hz}, 1 \mathrm{H}, \mathrm{Ar}), 7.82$ (d, $J=8.0 \mathrm{~Hz}, 1 \mathrm{H}, \mathrm{Ar}), 8.23$ (d, $J=8.6 \mathrm{~Hz}, 1 \mathrm{H}, \mathrm{Ar}) ;{ }^{13} \mathrm{C}-\mathrm{NMR}\left(125 \mathrm{MHz}, \mathrm{CDCl}_{3}\right)$ $\delta: 21.8,30.0$ (3C), 45.1, 45.4, 54.2, 120.7, 121.9, 122.3, 123.8, 123.9, 124.7, 124.8, 127.2, 129.0, 129.9, 136.0, 137.9, 139.7, 140.4, 142.3, 147.5; HRMS (FAB): $m / z$ calcd for $\mathrm{C}_{23} \mathrm{H}_{24} \mathrm{~N}_{3} \mathrm{~S}_{2}[\mathrm{M}+\mathrm{H}]^{+}$406.1412; found: 406.1407 .

Compound $32 \mathrm{~m}$. Using the general procedure as described for $\mathbf{2 5 a}$, compound 30m $(35.8 \mathrm{mg}, 0.09 \mathrm{mmol}$ ) was allowed to react for $3 \mathrm{~h}$. Purification by flash chromatography over aluminum oxide with $n$-hexane-EtOAc $(9: 1$ to $7: 3)$ gave the title compound $\mathbf{3 2 m}$ as pale yellow solid (26.9 $\mathrm{mg}, 86 \%$ ): $\mathrm{mp} 198-200{ }^{\circ} \mathrm{C}$ (from 
$\mathrm{MeOH}-\mathrm{CHCl}_{3}-n$-hexane); IR (neat) $\mathrm{cm}^{-1}: 1615(\mathrm{C}=\mathrm{N}), 1567(\mathrm{C}=\mathrm{N})$; ${ }^{1} \mathrm{H}-\mathrm{NMR}$ $\left(500 \mathrm{MHz}, \mathrm{CDCl}_{3}-\mathrm{CD}_{3} \mathrm{OD}\right) \delta: 1.95-2.00\left(\mathrm{~m}, 2 \mathrm{H}, \mathrm{CH}_{2}\right), 3.69(\mathrm{t}, J=5.4 \mathrm{~Hz}, 2 \mathrm{H}$, $\mathrm{CH}_{2}$ ), $4.01\left(\mathrm{t}, J=6.0 \mathrm{~Hz}, 2 \mathrm{H}, \mathrm{CH}_{2}\right), 7.25$ (br s, 1H, NH), 7.32-7.37 (m, 3H, Ar), 7.54 (dd, $J=8.6,1.7 \mathrm{~Hz}, 1 \mathrm{H}, \mathrm{Ar}), 7.58(\mathrm{~s}, 1 \mathrm{H}, \mathrm{Ar}), 7.77$ (t, $J=4.0 \mathrm{~Hz}, 1 \mathrm{H}, \mathrm{Ar})$, $7.82(\mathrm{~d}, J=7.4 \mathrm{~Hz}, 1 \mathrm{H}, \mathrm{Ar}), 8.23$ (d, $J=8.6 \mathrm{~Hz}, 1 \mathrm{H}, \mathrm{Ar}) ;{ }^{13} \mathrm{C}-\mathrm{NMR}(125 \mathrm{MHz}$, $\left.\mathrm{CDCl}_{3}-\mathrm{CD}_{3} \mathrm{OD}\right) \delta$ : 20.9, 43.9, 44.9, 120.9, 121.0, 122.3, 123.9, 124.2, 124.7, $124.9,125.9,129.4,129.6,136.6,139.7,140.3,141.8,146.4,153.2$; HRMS (FAB): $m / z$ calcd for $\mathrm{C}_{19} \mathrm{H}_{16} \mathrm{~N}_{3} \mathrm{~S}_{2}[\mathrm{M}+\mathrm{H}]^{+} 350.0786$; found: 350.0785 .

\subsubsection{Synthesis of 3,4-Dihydro-9-(1H-pyrazol-1-yl)-2H, 6H-pyrimido[1,2-c][1,3]benzothiazin-6-imine (32n)}

$N$-(tert-Butyl)-3,4-dihydro-9-(1H-pyrazol-1-yl)-2H,6H-pyrimido[1,2-c][1,3] benzothiazin-6-imine (30n). To a solution of $N$-(tert-butyl)-9-bromo-3,4-dihydro2H,6H-pyrimido[1,2-c][1,3]benzothiazin-6-imine 22k (52.8 mg, $0.15 \mathrm{mmol})$, pyrazole (12.3 mg, $0.18 \mathrm{mmol}), \mathrm{CuCl}(1.5 \mathrm{mg}, 0.015 \mathrm{mmol})$ and $\mathrm{K}_{2} \mathrm{CO}_{3}(21.8 \mathrm{mg}$, $0.16 \mathrm{~mol})$ in $N$-methylpyrrolidone $(0.3 \mathrm{~mL})$ was added acetylacetone $(3.8 \mu \mathrm{L}$, $0.038 \mathrm{mmol}$ ) under an Ar atmosphere. After being stirred at $130{ }^{\circ} \mathrm{C}$ for $19 \mathrm{~h}$, EtOAc was added. The organic layers were washed with $\mathrm{H}_{2} \mathrm{O}$, and dried over $\mathrm{MgSO}_{4}$. After concentration, the residue was purified by flash chromatography over aluminum oxide with $n$-hexane-EtOAc (7:3) to give the title compound 30n as colorless solid (39.8 mg, $71 \%$ ): $\mathrm{mp} 132-133{ }^{\circ} \mathrm{C}$ (from $\mathrm{CHCl}_{3}-n$-hexane); IR (neat) $\mathrm{cm}^{-1}: 1597$ $(\mathrm{C}=\mathrm{N}) ;{ }^{1} \mathrm{H}-\mathrm{NMR}\left(400 \mathrm{MHz}, \mathrm{CDCl}_{3}\right) \delta: 1.39\left(\mathrm{~s}, 9 \mathrm{H}, 3 \times \mathrm{CH}_{3}\right), 1.90-1.96(\mathrm{~m}, 2 \mathrm{H}$, $\left.\mathrm{CH}_{2}\right), 3.63\left(\mathrm{t}, J=5.6 \mathrm{~Hz}, 2 \mathrm{H}, \mathrm{CH}_{2}\right), 3.88\left(\mathrm{t}, J=6.2 \mathrm{~Hz}, 2 \mathrm{H}, \mathrm{CH}_{2}\right), 6.48$ (dd, $J=2.7,1.8 \mathrm{~Hz}, 1 \mathrm{H}, \mathrm{Ar}), 7.47(\mathrm{dd}, J=8.8,2.2 \mathrm{~Hz}, 1 \mathrm{H}, \mathrm{Ar}), 7.56(\mathrm{~d}, J=2.2 \mathrm{~Hz}$, $1 \mathrm{H}, \mathrm{Ar}), 7.73(\mathrm{~d}, J=1.8 \mathrm{~Hz}, 1 \mathrm{H}, \mathrm{Ar}), 7.94(\mathrm{~d}, J=2.7 \mathrm{~Hz}, 1 \mathrm{H}, \mathrm{Ar}), 8.28$ (d, $J=8.8 \mathrm{~Hz}, 1 \mathrm{H}, \mathrm{Ar}) ;{ }^{13} \mathrm{C}-\mathrm{NMR}\left(100 \mathrm{MHz}, \mathrm{CDCl}_{3}\right) \delta: 21.8,30.0$ (3C), 45.0, 45.4, 54.2, 108.2, 114.3, 115.9, 125.4, 126.7, 129.9, 130.8, 137.7, 141.0, 141.7, 147.3; HRMS (FAB): $m / z$ calcd for $\mathrm{C}_{18} \mathrm{H}_{22} \mathrm{~N}_{5} \mathrm{~S}[\mathrm{M}+\mathrm{H}]^{+}$340.1596; found: 340.1598 .

Compound 32n. Using the general procedure as described for 25a, compound 30n ( $21.6 \mathrm{mg}, 0.064 \mathrm{mmol}$ ) was allowed to react for $1 \mathrm{~h}$. Purification by preparative TLC over aluminum oxide with $\mathrm{CHCl}_{3}$ gave the title compound $\mathbf{3 2 n}$ as colorless solid $(16.2 \mathrm{mg}, 89 \%)$ : $\mathrm{mp} 158-159{ }^{\circ} \mathrm{C}$ (from $\mathrm{CHCl}_{3}-n$-hexane); IR (neat) $\mathrm{cm}^{-1}: 1615(\mathrm{C}=\mathrm{N}), 1561(\mathrm{C}=\mathrm{N}) ;{ }^{1} \mathrm{H}-\mathrm{NMR}\left(400 \mathrm{MHz}, \mathrm{CDCl}_{3}\right) \delta: 1.96-2.02$ $\left(\mathrm{m}, 2 \mathrm{H}, \mathrm{CH}_{2}\right), 3.70\left(\mathrm{t}, J=5.6 \mathrm{~Hz}, 2 \mathrm{H}, \mathrm{CH}_{2}\right), 4.03\left(\mathrm{t}, J=6.2 \mathrm{~Hz}, 2 \mathrm{H}, \mathrm{CH}_{2}\right), 6.49$ (dd, $J=2.6,1.8 \mathrm{~Hz}, 1 \mathrm{H}, \mathrm{Ar}), 7.27(\mathrm{~s}, 1 \mathrm{H}, \mathrm{NH}), 7.48-7.51$ (m, 2H, Ar), 7.74 (d, $J=1.5 \mathrm{~Hz}, 1 \mathrm{H}, \mathrm{Ar}), 7.95(\mathrm{~d}, J=2.7 \mathrm{~Hz}, 1 \mathrm{H}, \mathrm{Ar}), 8.31(\mathrm{~d}, J=9.5 \mathrm{~Hz}, 1 \mathrm{H}, \mathrm{Ar})$; ${ }^{13} \mathrm{C}-\mathrm{NMR}\left(100 \mathrm{MHz}, \mathrm{CDCl}_{3}\right) \delta: 21.0,43.8,44.9,108.4,113.3,116.1,124.3$, $126.7,130.3,130.6,141.3,141.8,145.9,152.8$; HRMS (FAB): $\mathrm{m} / \mathrm{z}$ calcd for $\mathrm{C}_{14} \mathrm{H}_{14} \mathrm{~N}_{5} \mathrm{~S}[\mathrm{M}+\mathrm{H}]^{+}$284.0970; found: 284.0966. 


\subsubsection{Synthesis of 3,4-Dihydro-9-(1H-imidazol-1-yl)- 2H,6H-pyrimido[1,2-c][1,3]benzothiazin-6-imine (32o)}

$N$-(tert-Butyl)-3,4-dihydro-9-(1H-imidazol-1-yl)-2H,6H-pyrimido[1,2-c][1,3] benzothiazin-6-imine (30o). Using the general procedure as described for 30n, $\mathrm{N}$-(tert-butyl)-9-bromo-3,4-dihydro-2H,6H-pyrimido[1,2-c][1,3]benzothiazin-6imine 22k (52.8 $\mathrm{mg}, 0.15 \mathrm{mmol})$ was allowed to react with imidazole $(12.3 \mathrm{mg}$, $0.18 \mathrm{mmol}$ ) for $3 \mathrm{~h}$. Purification by flash chromatography over aluminum oxide with $n$-hexane-EtOAc $(1: 1$ to $0: 1)$ gave the title compound 30o as colorless solid (25.8 mg, $51 \%$ ): $\mathrm{mp} 170-171{ }^{\circ} \mathrm{C}$ (from $\mathrm{CHCl}_{3}-n$-hexane); IR (neat) $\mathrm{cm}^{-1}: 1599$ $(\mathrm{C}=\mathrm{N}) ;{ }^{1} \mathrm{H}-\mathrm{NMR}\left(400 \mathrm{MHz}, \mathrm{CDCl}_{3}\right) \delta: 1.40\left(\mathrm{~s}, 9 \mathrm{H}, 3 \times \mathrm{CH}_{3}\right), 1.91-1.96(\mathrm{~m}, 2 \mathrm{H}$, $\left.\mathrm{CH}_{2}\right), 3.64\left(\mathrm{t}, J=5.6 \mathrm{~Hz}, 2 \mathrm{H}, \mathrm{CH}_{2}\right), 3.89\left(\mathrm{t}, J=6.2 \mathrm{~Hz}, 2 \mathrm{H}, \mathrm{CH}_{2}\right), 7.15(\mathrm{~d}$, $J=2.3 \mathrm{~Hz}, 1 \mathrm{H}, \mathrm{Ar}), 7.22(\mathrm{dd}, J=8.8,2.3 \mathrm{~Hz}, 1 \mathrm{H}, \mathrm{Ar}), 7.22(\mathrm{~s}, 1 \mathrm{H}, \mathrm{Ar}), 7.30$ (s, 1H, Ar), 7.90 (s, 1H, Ar), 8.32 (d, $J=8.8 \mathrm{~Hz}, 1 \mathrm{H}, \mathrm{Ar}) ;{ }^{13} \mathrm{C}-\mathrm{NMR}(100 \mathrm{MHz}$, $\mathrm{CDCl}_{3}$ ) $\delta: 21.8,30.0$ (3C), 45.0, 45.5, 54.3, 116.2, 117.7, 118.4, 126.5, 130.5, $130.8,131.3,135.3,136.9,138.4,146.9$; HRMS (FAB): $m / z$ calcd for $\mathrm{C}_{18} \mathrm{H}_{22} \mathrm{~N}_{5} \mathrm{~S}$ $[\mathrm{M}+\mathrm{H}]^{+}$340.1596; found: 340.1598 .

Compound 320. Using the general procedure as described for 25a, compound 30 o $(20.6 \mathrm{mg}, 0.061 \mathrm{mmol}$ ) was allowed to react for $1 \mathrm{~h}$. Purification by preparative TLC over aluminum oxide with EtOAc-MeOH (9:1) gave the title compound 32o as colorless solid $\left(9.7 \mathrm{mg}, 56 \%\right.$ ): $\mathrm{mp} 183-185{ }^{\circ} \mathrm{C}$ (from $\mathrm{CHCl}_{3}-n$ hexane); IR (neat) $\mathrm{cm}^{-1}$ : $1622(\mathrm{C}=\mathrm{N}), 1561(\mathrm{C}=\mathrm{N}) ;{ }^{1} \mathrm{H}-\mathrm{NMR}\left(400 \mathrm{MHz}, \mathrm{CDCl}_{3}\right)$ $\delta: 1.97-2.03\left(\mathrm{~m}, 2 \mathrm{H}, \mathrm{CH}_{2}\right), 3.71\left(\mathrm{t}, J=5.7 \mathrm{~Hz}, 2 \mathrm{H}, \mathrm{CH}_{2}\right), 4.04(\mathrm{t}, J=6.1 \mathrm{~Hz}, 2 \mathrm{H}$, $\mathrm{CH}_{2}$ ), 7.07 (d, $\left.J=2.2 \mathrm{~Hz}, 1 \mathrm{H}, \mathrm{Ar}\right), 7.22(\mathrm{~s}, 1 \mathrm{H}, \mathrm{Ar}), 7.25$ (dd, $J=8.8,2.2 \mathrm{~Hz}$, 1H, Ar), 7.29 (s, 1H, Ar), 7.89 (s, 1H, Ar), 8.36 (d, J = 8.8 Hz, 1H, Ar); ${ }^{13} \mathrm{C}-\mathrm{NMR}$ $\left(125 \mathrm{MHz}, \mathrm{CDCl}_{3}\right) \delta: 20.9,43.8,44.9,115.2,117.7,118.6,125.5,130.9,131.0$, 131.1, 135.3, 138.8, 145.6, 152.2; HRMS (FAB): $m / z$ calcd for $\mathrm{C}_{14} \mathrm{H}_{14} \mathrm{~N}_{5} \mathrm{~S}$ $[\mathrm{M}+\mathrm{H}]^{+}$284.0970; found: 284.0966 .

\subsubsection{Synthesis of 2,3-Dihydro-5H-imidazo[1,2-c][1,3] benzothiazin-5-imine (36a)}

Using the general procedure as described for 25a, $N$-(tert-butyl)-2,3-dihydroimidazo[1,2-c][1,3]benzothiazin-5-imine 35a $(18.4 \mathrm{mg}, 0.07 \mathrm{mmol})$ was allowed to

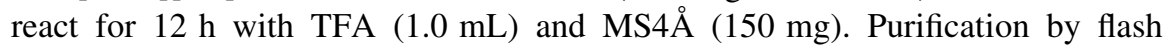
chromatography over silica gel with $n$-hexane-EtOAc $(1: 1)$ gave the title compound 36a as colorless solid (11.1 mg, $78 \%$ ): $\mathrm{mp} 176-178{ }^{\circ} \mathrm{C}$ (from $\mathrm{CHCl}_{3}-n$ hexane); IR (neat) $\mathrm{cm}^{-1}: 1621(\mathrm{C}=\mathrm{N}), 1585(\mathrm{C}=\mathrm{N}) ;{ }^{1} \mathrm{H}-\mathrm{NMR}\left(500 \mathrm{MHz}, \mathrm{CDCl}_{3}\right)$ $\delta: 4.11\left(4 \mathrm{H}, \mathrm{s}, 2 \times \mathrm{CH}_{2}\right), 5.82(1 \mathrm{H}$, br s, NH$), 7.12(1 \mathrm{H}, \mathrm{d}, J=8.0 \mathrm{~Hz}, \mathrm{Ar}), 7.24-$ $7.28(1 \mathrm{H}, \mathrm{m}, \mathrm{Ar}), 7.38-7.42(1 \mathrm{H}, \mathrm{m}, \mathrm{Ar}), 8.20(1 \mathrm{H}, \mathrm{dd}, J=7.7,1.4 \mathrm{~Hz}, \mathrm{Ar}) ;{ }^{13} \mathrm{C}-$ 
NMR (125 MHz, $\left.\mathrm{CDCl}_{3}\right) \delta: 47.3,52.9,120.8,123.8,126.5,129.1,132.0,132.4$, 150.0, 154.0; HRMS (FAB): $m / z$ calcd for $\mathrm{C}_{10} \mathrm{H}_{10} \mathrm{~N}_{3} \mathrm{~S}[\mathrm{M}+\mathrm{H}]^{+}$204.0595; found: 204.0600 .

\subsubsection{Synthesis of $6 \mathrm{H}, 8 \mathrm{H}$-Quinazolino[3,2-c][1,3] benzothiazin-6-imine (36b)}

$N$-(tert-Butyl)-6H,8H-quinazolino[3,2-c][1,3]benzothiazin-6-imine (35b). To a solution of 2-fluorobenzaldehyde $(1.41 \mathrm{~g}, 11.39 \mathrm{mmol})$ in $t$-BuOH $(38 \mathrm{~mL})$ was added 2-aminobenzylamine $33 \mathrm{~b}(1.53 \mathrm{~g}, 12.53 \mathrm{mmol})$. The mixture was stirred at $80{ }^{\circ} \mathrm{C}$ for $30 \mathrm{~min}$, and then $\mathrm{K}_{2} \mathrm{CO}_{3}(4.73 \mathrm{~g}, 34.18 \mathrm{mmol})$ and $\mathrm{I}_{2}(3.61 \mathrm{~g}$, $14.24 \mathrm{mmol}$ ) were added. After being stirred at same temperature for $4 \mathrm{~h}$, the mixture was quenched with sat. $\mathrm{Na}_{2} \mathrm{SO}_{3}$. The organic layer was separated and concentrated. The resulting solid was dissolved with $\mathrm{H}_{2} \mathrm{O}$ and $\mathrm{CHCl}_{3}$, and then $\mathrm{pH}$ was adjusted to $12-14$ with $5 \mathrm{~N} \mathrm{NaOH}$. The whole was extracted with $\mathrm{CHCl}_{3}$. The extract was washed with brine, dried over $\mathrm{Na}_{2} \mathrm{SO}_{4}$. To a mixture of resulting residue in DMAc $(7.4 \mathrm{~mL})$ were added $\mathrm{KO}$ - $\mathrm{Bu}(496 \mathrm{mg}, 4.42 \mathrm{mmol})$ and tertbutylisothiocyanate $(0.56 \mathrm{~mL}, 4.42 \mathrm{mmol})$ under an $\mathrm{N}_{2}$ atmosphere. After being stirred at $80{ }^{\circ} \mathrm{C}$ for $2.5 \mathrm{~h}$, sat. $\mathrm{NH}_{4} \mathrm{Cl}$ was added. The whole was extracted with EtOAc. The extract was washed with brine, and dried over $\mathrm{Na}_{2} \mathrm{SO}_{4}$. After concentration, the residue was purified by flash chromatography over silica gel with $n$ hexane-EtOAc (1:0 to $9: 1)$ to give the title compound $35 \mathbf{b}$ as yellow solid (114.1 mg, $3.1 \%$ over 2 steps): $\mathrm{mp} 92.2{ }^{\circ} \mathrm{C}$; IR (neat) $\mathrm{cm}^{-1}: 1588(\mathrm{C}=\mathrm{N}) ;{ }^{1} \mathrm{H}-$ NMR $\left(300 \mathrm{MHz}, \mathrm{CDCl}_{3}\right) \delta: 1.42\left(9 \mathrm{H}, \mathrm{s}, 3 \times \mathrm{CH}_{3}\right), 5.10\left(2 \mathrm{H}, \mathrm{s}, \mathrm{CH}_{2}\right), 7.08-7.23$ (3H, m, Ar), 7.27-7.40 (4H, m, Ar), $8.43(1 \mathrm{H}, \mathrm{dd}, J=8.0,1.4 \mathrm{~Hz}, \mathrm{Ar}) ;{ }^{13} \mathrm{C}-\mathrm{NMR}$ $\left(75 \mathrm{MHz}, \mathrm{CDCl}_{3}\right) \delta: 29.9$ (3C), 46.2, 54.7, 124.0, 124.8, 124.9, 125.4, 125.8, 126.4, 127.7, 128.3, 129.0, 129.4, 130.7, 138.3, 141.1, 148.3; Anal. calcd for $\mathrm{C}_{19} \mathrm{H}_{19} \mathrm{~N}_{3} \mathrm{~S}$ : C, 70.99; H, 5.96; N, 13.07. Found: C, 71.05; H, 5.99; N, 12.91.

Compound 36b. TFA $(0.5 \mathrm{~mL})$ was added to 35b (100 mg, $0.311 \mathrm{mmol})$. After being stirred under reflux for $30 \mathrm{~min}$, the mixture was added dropwise to $\mathrm{Et}_{3} \mathrm{~N}$ at $0{ }^{\circ} \mathrm{C}$ to adjust $\mathrm{pH}$ to $8-9$. The whole was extracted with EtOAc. The extract was washed with sat. $\mathrm{NaHCO}_{3}$ aq., brine, and dried over $\mathrm{Na}_{2} \mathrm{SO}_{4}$. After concentration, the residue was purified by preparative TLC over aluminum oxide with $n$-hexaneEtOAc (9:1) to give the title compound $36 \mathrm{~b}$ as colorless solid (13 mg, $16 \%): \mathrm{mp}$ 133-135 ${ }^{\circ} \mathrm{C}$ (from $\mathrm{CHCl}_{3}-n$-hexane); IR (neat) $\mathrm{cm}^{-1}: 1594(\mathrm{C}=\mathrm{N}), 1541(\mathrm{C}=\mathrm{N})$; ${ }^{1} \mathrm{H}-\mathrm{NMR}\left(500 \mathrm{MHz}, \mathrm{CDCl}_{3}\right) \delta: 5.27\left(2 \mathrm{H}, \mathrm{s}, \mathrm{CH}_{2}\right), 7.08-7.13(2 \mathrm{H}, \mathrm{m}, \mathrm{Ar}), 7.16$ $(1 \mathrm{H}, \mathrm{t}, J=7.2 \mathrm{~Hz}, \mathrm{Ar}), 7.26-7.34(3 \mathrm{H}, \mathrm{m}, \mathrm{Ar}), 7.39(1 \mathrm{H}, \mathrm{td}, J=6.9,1.1 \mathrm{~Hz}, \mathrm{Ar})$, $7.59\left(1 \mathrm{H}\right.$, br s, NH), $8.50(1 \mathrm{H}, \mathrm{d}, J=8.0 \mathrm{~Hz}, \mathrm{Ar}) ;{ }^{13} \mathrm{C}-\mathrm{NMR}\left(125 \mathrm{MHz}, \mathrm{CDCl}_{3}\right)$ $\delta: 45.1,123.0,123.8,125.4,125.6,126.3,126.5,126.6,128.5,129.2,129.5,131.1$, 140.1, 146.3, 153.3; HRMS (FAB): $m / z$ calcd for $\mathrm{C}_{15} \mathrm{H}_{12} \mathrm{~N}_{3} \mathrm{~S}[\mathrm{M}+\mathrm{H}]^{+} 266.0752$; found: 266.0750 . 


\subsubsection{Synthesis of ( \pm )-3,4-Dihydro-3-methyl-2H, 6H-pyrimido[1,2-c][1,3]benzothiazin-6-imine (36c)}

2-(2-Fluorophenyl)-5-methyl-1,4,5,6-tetrahydropyrimidine (34c). 2-Fluorobenzaldehyde $(0.62 \mathrm{~g}, 5.0 \mathrm{mmol})$ was subjected to the general procedure for $\mathbf{1 8 j}$ using 2-methylpropylenediamine $33 \mathrm{c}(0.48 \mathrm{~g}, 5.5 \mathrm{mmol})$ to give the title compound $\mathbf{3 4 c}$ as colorless crystals $(0.72 \mathrm{~g}, 75 \%)$ : $\mathrm{mp} 98-99{ }^{\circ} \mathrm{C}$ (from $\mathrm{CHCl}_{3}-n$-hexane); IR (neat) $\mathrm{cm}^{-1}: 1628(\mathrm{C}=\mathrm{N}) ;{ }^{1} \mathrm{H}-\mathrm{NMR}\left(500 \mathrm{MHz}, \mathrm{CDCl}_{3}\right) \delta: 1.01(3 \mathrm{H}, \mathrm{d}$, $\left.J=6.9 \mathrm{~Hz}, \mathrm{CH}_{3}\right), 1.92-1.99(1 \mathrm{H}, \mathrm{m}, \mathrm{CH}), 3.06(2 \mathrm{H}, \mathrm{dd}, J=13.2,9.7 \mathrm{~Hz}$, $2 \times \mathrm{CH}), 3.52(2 \mathrm{H}, \mathrm{dd}, J=13.2,3.4 \mathrm{~Hz}, 2 \times \mathrm{CH}), 5.27(1 \mathrm{H}$, br s, NH), $7.04(1 \mathrm{H}$, $\mathrm{dd}, J=11.7,8.3 \mathrm{~Hz}, \mathrm{Ar}), 7.15(1 \mathrm{H}, \mathrm{t}, J=7.4 \mathrm{~Hz}, \mathrm{Ar}), 7.30-7.35(1 \mathrm{H}, \mathrm{m}, \mathrm{Ar})$, $7.81(1 \mathrm{H}, \mathrm{td}, J=7.4,1.7 \mathrm{~Hz}, \mathrm{Ar}) ;{ }^{13} \mathrm{C}-\mathrm{NMR}\left(125 \mathrm{MHz}, \mathrm{CDCl}_{3}\right) \delta: 16.8,25.2$, $49.4(2 \mathrm{C}), 115.9(\mathrm{~d}, J=24.0 \mathrm{~Hz}), 124.2,124.3(\mathrm{~d}, J=3.6 \mathrm{~Hz}), 130.6$ (d, $J=3.6 \mathrm{~Hz}), 130.8(\mathrm{~d}, J=8.4 \mathrm{~Hz}), 151.3,160.1(\mathrm{~d}, J=247.1 \mathrm{~Hz}) ;{ }^{19} \mathrm{~F}-\mathrm{NMR}$ $\left(500 \mathrm{MHz}, \mathrm{CDCl}_{3}\right) \delta$ : -117.1 ; HRMS (FAB): $m / z$ calcd for $\mathrm{C}_{11} \mathrm{H}_{14} \mathrm{FN}_{2}[\mathrm{M}+\mathrm{H}]^{+}$ 193.1141; found: 193.1136 .

( \pm ) - $N$-(-(tert-Butyl)-3,4-dihydro-3-methyl-2H,6H-pyrimido[1,2-c][1,3]benzothiazin-6-imine $\mathbf{( 3 5} \mathbf{c})$. Using the general procedure as described for $22 \mathbf{e}$, compound 34c $(384.5 \mathrm{mg}, 2.0 \mathrm{mmol})$ was allowed to react at $80{ }^{\circ} \mathrm{C}$ for $2 \mathrm{~h}$. Purification by flash chromatography over aluminum oxide with $n$-hexane-EtOAc (1:0 to 95:5) gave the title compound 35c as colorless solid (288.4 mg, $50 \%): \mathrm{mp}$ 60-62 ${ }^{\circ} \mathrm{C}$ (from $n$-hexane); IR (neat) $\mathrm{cm}^{-1}: 1598(\mathrm{C}=\mathrm{N}), 1570(\mathrm{C}=\mathrm{N}) ;{ }^{13} \mathrm{C}-\mathrm{NMR}$ $\left(500 \mathrm{MHz}, \mathrm{CDCl}_{3}\right) \delta: 1.05\left(3 \mathrm{H}, \mathrm{d}, J=6.3 \mathrm{~Hz}, \mathrm{CH}_{3}\right), 1.39\left(9 \mathrm{H}, \mathrm{s}, 3 \times \mathrm{CH}_{3}\right)$, 1.91-1.99 (1H, m, CH), 3.09-3.17 (2H, m, $\left.\mathrm{CH}_{2}\right), 3.72(1 \mathrm{H}, \mathrm{dt}, J=15.5,3.7 \mathrm{~Hz}$, $\mathrm{CH}), 4.19(1 \mathrm{H}, \mathrm{dt}, J=13.7,3.7 \mathrm{~Hz}, \mathrm{CH}), 7.11(1 \mathrm{H}, \mathrm{d}, J=8.0 \mathrm{~Hz}, \mathrm{Ar}), 7.19(1 \mathrm{H}$, $\mathrm{t}, J=8.0 \mathrm{~Hz}, \mathrm{Ar}), 7.30(1 \mathrm{H}, \mathrm{t}, J=8.0 \mathrm{~Hz}, \mathrm{Ar}), 8.19(1 \mathrm{H}, \mathrm{d}, J=8.0 \mathrm{~Hz}, \mathrm{Ar})$; ${ }^{13} \mathrm{C}-\mathrm{NMR}\left(125 \mathrm{MHz}, \mathrm{CDCl}_{3}\right.$ ) $\delta: 16.7,26.9,30.0$ (3C), 51.6, 52.4, 54.2, 124.4, 126.0, 127.7, 128.5, 129.1, 130.1, 138.4, 147.6; HRMS (FAB): $\mathrm{m} / \mathrm{z}$ calcd for $\mathrm{C}_{16} \mathrm{H}_{22} \mathrm{~N}_{3} \mathrm{~S}[\mathrm{M}+\mathrm{H}]^{+}$288.1534; found: 288.1535.

Compound 36c. Using the general procedure as described for 25a, compound 35c $(57.5 \mathrm{mg}, 0.20 \mathrm{mmol})$ was allowed to react for $1 \mathrm{~h}$. Purification by flash chromatography over aluminum oxide with $n$-hexane-EtOAc $(9: 1)$ gave the title compound 36c as colorless solid $\left(36.7 \mathrm{mg}, 79 \%\right.$ ): $\mathrm{mp} 82-84{ }^{\circ} \mathrm{C}$ (from $\mathrm{CHCl}_{3}-n$ hexane); IR (neat) $\mathrm{cm}^{-1}: 1621(\mathrm{C}=\mathrm{N}), 1574(\mathrm{C}=\mathrm{N}) ;{ }^{1} \mathrm{H}-\mathrm{NMR}\left(500 \mathrm{MHz}, \mathrm{CDCl}_{3}\right)$ $\delta: 1.09\left(3 \mathrm{H}, \mathrm{d}, J=6.3 \mathrm{~Hz}, \mathrm{CH}_{3}\right), 1.96-2.08(1 \mathrm{H}, \mathrm{m}, \mathrm{CH}), 3.19(1 \mathrm{H}, \mathrm{dd}, J=15.8$, $10.6 \mathrm{~Hz}, \mathrm{CH}), 3.27(1 \mathrm{H}, \mathrm{dd}, J=13.0,10.6 \mathrm{~Hz}, \mathrm{CH}), 3.80(1 \mathrm{H}, \mathrm{ddd}, J=15.8,4.5$, $3.2 \mathrm{~Hz}, \mathrm{CH}), 4.37(1 \mathrm{H}, \mathrm{ddd}, J=13.0,4.5,3.2 \mathrm{~Hz}, \mathrm{CH}), 7.04(1 \mathrm{H}, \mathrm{d}, J=7.4 \mathrm{~Hz}$, Ar), 7.18-7.25 (2H, m, Ar, NH), $7.33(1 \mathrm{H}, \mathrm{td}, J=7.4,1.4 \mathrm{~Hz}, \mathrm{Ar}), 8.23(1 \mathrm{H}, \mathrm{dd}$, $J=8.3,1.4 \mathrm{~Hz}, \mathrm{Ar}) ;{ }^{13} \mathrm{C}-\mathrm{NMR}\left(125 \mathrm{MHz}, \mathrm{CDCl}_{3}\right) \delta: 16.4,26.1,49.9,52.2$, $123.5,126.3,126.6,128.8,128.9,130.6,146.2,153.4$; Anal. calcd for $\mathrm{C}_{12} \mathrm{H}_{13} \mathrm{~N}_{3} \mathrm{~S}$ : C, 62.31; H, 5.66; N, 18.17. Found: C, 62.04; H, 5.75; N, 17.88. 


\subsubsection{Synthesis of 3,4-Dihydro-3,3-dimethyl-2H, 6H-pyrimido[1,2-c][1,3]benzothiazin-6-imine (36d)}

2-(2-Fluorophenyl)-5,5-dimethyl-1,4,5,6-tetrahydropyrimidine (34d). 2-Fluorobenzaldehyde $(0.62 \mathrm{~g}, 5.0 \mathrm{mmol})$ was subjected to the general procedure for $\mathbf{1 8} \mathbf{j}$ using 2,2-dimethylpropylenediamine $33 d(0.56 \mathrm{~g}, 5.5 \mathrm{mmol})$ to give the title compound $34 \mathrm{~d}$ as colorless crystals $(0.82 \mathrm{~g}, 79 \%)$ : mp $150-153{ }^{\circ} \mathrm{C}$ (from $\mathrm{CHCl}_{3}-$ $n$-hexane); IR (neat) $\mathrm{cm}^{-1}: 1629(\mathrm{C}=\mathrm{N}) ;{ }^{1} \mathrm{H}-\mathrm{NMR}\left(400 \mathrm{MHz}, \mathrm{CDCl}_{3}\right) \delta: 1.02(6 \mathrm{H}$, s, $\left.2 \times \mathrm{CH}_{3}\right), 3.13\left(4 \mathrm{H}, \mathrm{s}, 2 \times \mathrm{CH}_{2}\right), 5.14(1 \mathrm{H}$, br s, $\mathrm{NH}), 7.05(1 \mathrm{H}$, ddd, $J=11.7$, 7.8, 1.0 Hz, Ar), $7.15(1 \mathrm{H}, \mathrm{td}, J=7.8,1.0 \mathrm{~Hz}, \mathrm{Ar}), 7.30-7.35$ (1H, m, Ar), 7.81 $(1 \mathrm{H}, \mathrm{td}, J=7.8,2.0 \mathrm{~Hz}, \mathrm{Ar}) ;{ }^{13} \mathrm{C}-\mathrm{NMR}\left(100 \mathrm{MHz}, \mathrm{CDCl}_{3}\right) \delta: 25.0(2 \mathrm{C}), 26.2$, $54.3(2 \mathrm{C}), 115.8(\mathrm{~d}, J=23.2 \mathrm{~Hz}), 124.2,124.3(\mathrm{~d}, J=3.3 \mathrm{~Hz}), 130.6(\mathrm{~d}$, $J=4.1 \mathrm{~Hz}), \quad 130.8 \quad(\mathrm{~d}, \quad J=9.1 \mathrm{~Hz}), 150.5 \quad(\mathrm{~d}, \quad J=1.7 \mathrm{~Hz}), 160.2 \quad(\mathrm{~d}$, $J=247.5 \mathrm{~Hz}) ;{ }^{19} \mathrm{~F}-\mathrm{NMR}\left(500 \mathrm{MHz}, \mathrm{CDCl}_{3}\right) \delta:-117.3$; HRMS (FAB): $m / z$ calcd for $\mathrm{C}_{12} \mathrm{H}_{16} \mathrm{FN}_{2}[\mathrm{M}+\mathrm{H}]^{+}$207.1298; found: 207.1299 .

$\mathrm{N}$-(tert-Butyl)-3,4-dihydro-3,3-dimethyl-2H,6H-pyrimido[1,2-c][1,3]benzothiazin-6-imine (35d). Using the general procedure as described for $\mathbf{2 2 e}$, compound 34d $(412.5 \mathrm{mg}, 2.0 \mathrm{mmol})$ was allowed to react at $80{ }^{\circ} \mathrm{C}$ for $2 \mathrm{~h}$. Purification by flash chromatography over aluminum oxide with $n$-hexane-EtOAc (1:0 to 9:1) gave the title compound $35 \mathrm{~d}$ as colorless solid $(236.6 \mathrm{mg}, 39 \%): \mathrm{mp}$ 70-72 ${ }^{\circ} \mathrm{C}$ (from $n$-hexane); IR (neat) $\mathrm{cm}^{-1}$ : $1602(\mathrm{C}=\mathrm{N}), 1570(\mathrm{C}=\mathrm{N}) ;{ }^{1} \mathrm{H}-\mathrm{NMR}$ $\left(500 \mathrm{MHz}, \mathrm{CDCl}_{3}\right) \delta: 1.01\left(6 \mathrm{H}, \mathrm{s}, 2 \times \mathrm{CH}_{3}\right), 1.39\left(9 \mathrm{H}, \mathrm{s}, 3 \times \mathrm{CH}_{3}\right), 3.33(2 \mathrm{H}, \mathrm{s}$, $\left.\mathrm{CH}_{2}\right), 3.58\left(2 \mathrm{H}, \mathrm{s}, \mathrm{CH}_{2}\right), 7.12(1 \mathrm{H}, \mathrm{d}, J=8.0 \mathrm{~Hz}, \mathrm{Ar}), 7.20(1 \mathrm{H}, \mathrm{t}, J=8.0 \mathrm{~Hz}$, Ar), $7.31(1 \mathrm{H}, \mathrm{td}, J=8.0,1.1 \mathrm{~Hz}, \mathrm{Ar}), 8.21(1 \mathrm{H}, \mathrm{dd}, J=8.0,1.1 \mathrm{~Hz}, \mathrm{Ar}) ;{ }^{13} \mathrm{C}-$ NMR (125 MHz, $\mathrm{CDCl}_{3}$ ) $\delta: 24.8$ (2C), 28.5, 29.9 (3C), 54.2, 55.7, 57.4, 124.5, $126.0,127.5,128.5,129.1,130.1,138.7,146.7$; HRMS (FAB): $\mathrm{m} / z$ calcd for $\mathrm{C}_{17} \mathrm{H}_{24} \mathrm{~N}_{3} \mathrm{~S}[\mathrm{M}+\mathrm{H}]^{+}$302.1691; found: 302.1695 .

Compound 36d. Using the general procedure as described for 25a, compound 35d $(60.3 \mathrm{mg}, 0.20 \mathrm{mmol})$ was allowed to react for $1 \mathrm{~h}$. Purification by flash chromatography over aluminum oxide with $n$-hexane-EtOAc $(9: 1)$ gave the title compound 36d as colorless solid $\left(42.0 \mathrm{mg}, 86 \%\right.$ ): $\mathrm{mp} 113-114{ }^{\circ} \mathrm{C}$ (from $\mathrm{CHCl}_{3}-$ $n$-hexane); IR (neat) $\mathrm{cm}^{-1}$ : $1627(\mathrm{C}=\mathrm{N}), 1575(\mathrm{C}=\mathrm{N}) ;{ }^{1} \mathrm{H}-\mathrm{NMR}(500 \mathrm{MHz}$, $\left.\mathrm{CDCl}_{3}\right) \delta: 1.05\left(6 \mathrm{H}, \mathrm{s}, 2 \times \mathrm{CH}_{3}\right), 3.41\left(2 \mathrm{H}, \mathrm{s}, \mathrm{CH}_{2}\right), 3.74\left(2 \mathrm{H}, \mathrm{s}, \mathrm{CH}_{2}\right), 7.05(1 \mathrm{H}$, $\mathrm{dd}, J=7.6,1.1 \mathrm{~Hz}, \mathrm{Ar}), 7.21-7.25(2 \mathrm{H}, \mathrm{m}, \mathrm{Ar}, \mathrm{NH}), 7.34(1 \mathrm{H}, \mathrm{td}, J=7.6$, $1.4 \mathrm{~Hz}, \mathrm{Ar}), 8.26(1 \mathrm{H}, \mathrm{dd}, J=8.3,1.4 \mathrm{~Hz}, \mathrm{Ar}) ;{ }^{13} \mathrm{C}-\mathrm{NMR}\left(125 \mathrm{MHz}, \mathrm{CDCl}_{3}\right) \delta$ : 24.6 (2C), 27.9, 54.0, 57.2, 123.5, 126.3, 126.3, 128.8, 128.9, 130.6, 145.3, 153.8; HRMS (FAB): $m / z$ calcd for $\mathrm{C}_{13} \mathrm{H}_{16} \mathrm{~N}_{3} \mathrm{~S}[\mathrm{M}+\mathrm{H}]^{+} 246.1065$; found: 246.1069 . 


\subsubsection{Synthesis of 2,3,4,5-Tetrahydro-7H-1,3-diazepino [1,2-c][1,3]benzothiazin-7-imine (36e)}

$N$-(tert-Butoxycarbonyl)-2-(2-fluorophenyl)-4,5,6,7-tetrahydro-1,3-diazepine (37). To a solution of 2-fluorobenzaldehyde $(2.48 \mathrm{~g}, 20.0 \mathrm{mmol})$ in $t$-BuOH $(188 \mathrm{~mL})$ was added 1,4-diaminobutane 33e $(2.21 \mathrm{~mL}, 22.0 \mathrm{mmol})$. The mixture was stirred at $70{ }^{\circ} \mathrm{C}$ for $30 \mathrm{~min}$, and then $\mathrm{K}_{2} \mathrm{CO}_{3}(8.29 \mathrm{~g}, 60.0 \mathrm{mmol})$ and $\mathrm{I}_{2}$ ( $6.35 \mathrm{~g}, 25 \mathrm{mmol})$ were added. After being stirred at same temperature for $3 \mathrm{~h}$, the mixture was quenched with sat. $\mathrm{Na}_{2} \mathrm{SO}_{3}$. The organic layer was separated and concentrated. The resulting solid was dissolved with $\mathrm{H}_{2} \mathrm{O}$, and then $\mathrm{pH}$ was adjusted to $12-14$ with $2 \mathrm{~N} \mathrm{NaOH}$. The whole was extracted with $\mathrm{CHCl}_{3}$, and dried over $\mathrm{Na}_{2} \mathrm{SO}_{4}$. After concentration, $\mathrm{Et}_{3} \mathrm{~N}(8.67 \mathrm{~mL}, 60.0 \mathrm{mmol})$ and $\mathrm{Boc}_{2} \mathrm{O}$ (13.8 $\mathrm{mL}, 60.0 \mathrm{mmol}$ ) were added to the solution of residue in $\mathrm{CH}_{2} \mathrm{Cl}_{2}(100 \mathrm{~mL})$. After being stirred for $30 \mathrm{~min}$ at rt, sat. $\mathrm{NaHCO}_{3}$ was added. After being stirred at $\mathrm{rt}$ for $1 \mathrm{~h}$, the whole was extracted with $\mathrm{CHCl}_{3}$. The extract was washed with brine, and dried over $\mathrm{MgSO}_{4}$. After concentration, the residue was purified by column chromatography over silica gel with $n$-hexane-EtOAc $(4: 1)$ to give the title compound 37 as colorless solid $(2.18 \mathrm{~g}, 37 \%)$ : $\mathrm{mp} 63-65{ }^{\circ} \mathrm{C}$ (from $n$-hexane); IR (neat) $\mathrm{cm}^{-1}: 1710(\mathrm{C}=\mathrm{O}), 1631(\mathrm{C}=\mathrm{N}) ;{ }^{1} \mathrm{H}-\mathrm{NMR}\left(500 \mathrm{MHz}, \mathrm{CDCl}_{3}\right) \delta: 1.14(9 \mathrm{H}$, $\left.\mathrm{s}, 3 \times \mathrm{CH}_{3}\right), 1.66-1.70\left(2 \mathrm{H}, \mathrm{m}, \mathrm{CH}_{2}\right), 1.78-1.83\left(2 \mathrm{H}, \mathrm{m}, \mathrm{CH}_{2}\right), 3.61(2 \mathrm{H}$, br s, $\left.\mathrm{CH}_{2}\right), 3.76\left(2 \mathrm{H}, \mathrm{t}, J=5.2 \mathrm{~Hz}, \mathrm{CH}_{2}\right), 7.03(1 \mathrm{H}, \mathrm{dd}, J=11.2,8.3 \mathrm{~Hz}, \mathrm{Ar}), 7.15$ $(1 \mathrm{H}, \mathrm{td}, J=7.7,1.1 \mathrm{~Hz}, \mathrm{Ar}), 7.33-7.38(1 \mathrm{H}, \mathrm{m}, \mathrm{Ar}), 7.60(1 \mathrm{H}, \mathrm{t}, J=7.7 \mathrm{~Hz}, \mathrm{Ar})$; ${ }^{13} \mathrm{C}-\mathrm{NMR}\left(125 \mathrm{MHz}, \mathrm{CDCl}_{3}\right) \delta: 23.2,26.4,27.7$ (3C), 44.9, 50.7, 81.1, 115.7 (d, $J=21.6 \mathrm{~Hz}), 124.0(\mathrm{~d}, J=2.4 \mathrm{~Hz}), 126.5,130.9(\mathrm{~d}, J=2.4 \mathrm{~Hz}), 131.1(\mathrm{~d}$, $J=8.4 \mathrm{~Hz}), 152.8,154.8,160.5(\mathrm{~d}, J=250.7 \mathrm{~Hz}) ;{ }^{19} \mathrm{~F}-\mathrm{NMR}\left(500 \mathrm{MHz}, \mathrm{CDCl}_{3}\right)$ $\delta$ : -118.9 ; HRMS (FAB) $m / z$ calcd for $\mathrm{C}_{16} \mathrm{H}_{22} \mathrm{FN}_{2} \mathrm{O}_{2}[\mathrm{M}+\mathrm{H}]^{+} 293.1665$; found: 293.1669.

2-(2-Fluorophenyl)-4,5,6,7-tetrahydro-1H-1,3-diazepine (34e). To a solution of $37(877.1 \mathrm{mg}, 3.0 \mathrm{mmol})$ in $\mathrm{CH}_{2} \mathrm{Cl}_{2}(6.0 \mathrm{~mL})$ was added TFA $(6.0 \mathrm{~mL})$. The mixture was stirred under reflux for $2 \mathrm{~h}$, mixture was washed with $2 \mathrm{~N} \mathrm{NaOH}$. The organic phase was dried over $\mathrm{MgSO}_{4}$. After concentration, the residue was recrystallized from $\mathrm{CHCl}_{3}-n$-hexane to give the title compound $\mathbf{3 4} \mathbf{e}$ as colorless crystals (461.2 mg, $80 \%$ ): $\mathrm{mp} 92{ }^{\circ} \mathrm{C}$; IR (neat) $\mathrm{cm}^{-1}: 1627(\mathrm{C}=\mathrm{N})$; ${ }^{1} \mathrm{H}-\mathrm{NMR}$ $\left(500 \mathrm{MHz} \mathrm{CDCl}_{3}\right) \delta: 1.80-1.83\left(4 \mathrm{H}, \mathrm{m}, 2 \times \mathrm{CH}_{2}\right), 3.48\left(4 \mathrm{H}\right.$, br s, $\left.2 \times \mathrm{CH}_{2}\right)$, $4.86(1 \mathrm{H}$, br s, NH), 7.02-7.06 $(1 \mathrm{H}, \mathrm{m}, \mathrm{Ar}), 7.12(1 \mathrm{H}, \mathrm{td}, J=7.7,1.1 \mathrm{~Hz}, \mathrm{Ar})$, 7.30-7.34 (1H, m, Ar), $7.63(1 \mathrm{H}, \mathrm{td}, J=7.7,1.7 \mathrm{~Hz}, \mathrm{Ar}) ;{ }^{13} \mathrm{C}-\mathrm{NMR}(125 \mathrm{MHz}$, $\left.\mathrm{CDCl}_{3}\right) \delta: 28.4(2 \mathrm{C}), 47.9(2 \mathrm{C}), 115.7(\mathrm{~d}, J=22.8 \mathrm{~Hz}), 124.2(\mathrm{~d}, J=3.6 \mathrm{~Hz})$, $127.0(\mathrm{~d}, J=12.0 \mathrm{~Hz}), 130.9(\mathrm{~d}, J=8.4 \mathrm{~Hz}), 131.2(\mathrm{~d}, J=3.6 \mathrm{~Hz}), 157.2$, $160.4(\mathrm{~d}, J=247.1 \mathrm{~Hz}) ;{ }^{19} \mathrm{~F}-\mathrm{NMR}\left(500 \mathrm{MHz} \mathrm{CDCl}_{3}\right) \delta:-117.7$; HRMS (FAB) $m / z$ calcd for $\mathrm{C}_{11} \mathrm{H}_{14} \mathrm{FN}_{2}[\mathrm{M}+\mathrm{H}]^{+}$193.1141; found: 193.1140 .

$N$-(tert-Butyl)-7H-2,3,4,5-tetrahydro-1,3-diazepino[1,2-c][1,3]benzothiazin7-imine (35e). Using the general procedure as described for $25 \mathbf{e}$, compound $34 \mathbf{e}$ (192.2 $\mathrm{mg}, 1.0 \mathrm{mmol}$ ) was allowed to react at $\mathrm{rt}$ overnight. Purification by flash chromatography over silica gel with $n$-hexane-EtOAc (4:1) gave the title compound 
35e as a yellow oil $(50.3 \mathrm{mg}, 18 \%)$ : IR (neat) $\mathrm{cm}^{-1}: 1588(\mathrm{C}=\mathrm{N})$; ${ }^{1} \mathrm{H}-\mathrm{NMR}$ $\left(400 \mathrm{MHz} \mathrm{CDCl}_{3}\right) \delta: 1.37\left(9 \mathrm{H}, \mathrm{s}, 3 \times \mathrm{CH}_{3}\right), 1.87-1.93\left(4 \mathrm{H}, \mathrm{m}, 2 \times \mathrm{CH}_{2}\right), 3.82(2 \mathrm{H}$, $\left.\mathrm{t}, J=5.4 \mathrm{~Hz}, \mathrm{CH}_{2}\right), 3.88\left(2 \mathrm{H}, \mathrm{t}, J=5.4 \mathrm{~Hz}, \mathrm{CH}_{2}\right), 7.16-7.23(2 \mathrm{H}, \mathrm{m}, \mathrm{Ar}), 7.26-$ $7.31(1 \mathrm{H}, \mathrm{m}, \mathrm{Ar}), 7.84(1 \mathrm{H}, \mathrm{d}, J=7.1 \mathrm{~Hz}, \mathrm{Ar}) ;{ }^{13} \mathrm{C}-\mathrm{NMR}\left(100 \mathrm{MHz}, \mathrm{CDCl}_{3}\right) \delta: 23.3$, 24.5, 30.2 (3C), 48.3, 49.2, 53.8, 124.9, 126.3, 127.0, 129.4, 129.7, 133.5, 140.0, 152.2; HRMS (FAB) $\mathrm{m} / z$ calcd for $\mathrm{C}_{16} \mathrm{H}_{22} \mathrm{~N}_{3} \mathrm{~S}[\mathrm{M}+\mathrm{H}]^{+}$288.1534; found: 288.1540.

Compound 36e. Using the general procedure as described for 25a, compound 35e $(50.3 \mathrm{mg}, 0.18 \mathrm{mmol})$ was allowed to react for $2 \mathrm{~h}$. Purification by flash chromatography over aluminum oxide with $n$-hexane-EtOAc (4:1 to $2: 1)$ gave the title compound $36 \mathrm{e}$ as a colorless oil $(11.3 \mathrm{mg}, 27 \%)$ : IR (neat) $\mathrm{cm}^{-1}$ : 1638 $(\mathrm{C}=\mathrm{N}), 1578(\mathrm{C}=\mathrm{N})$; ${ }^{1} \mathrm{H}-\mathrm{NMR}\left(400 \mathrm{MHz}, \mathrm{CDCl}_{3}\right) \delta: 1.94-2.01 \quad(4 \mathrm{H}, \mathrm{m}$, $\left.2 \times \mathrm{CH}_{2}\right), 3.92\left(2 \mathrm{H}, \mathrm{t}, J=5.5 \mathrm{~Hz}, \mathrm{CH}_{2}\right), 3.96\left(2 \mathrm{H}, \mathrm{t}, J=5.6 \mathrm{~Hz}, \mathrm{CH}_{2}\right), 7.00$ $(1 \mathrm{H}$, br s, NH), 7.12-7.14 (1H, m, Ar $), 7.23-7.28(1 \mathrm{H}, \mathrm{m}, \mathrm{Ar}), 7.31-7.35(1 \mathrm{H}, \mathrm{m}$, Ar), $7.90(1 \mathrm{H}, \mathrm{dd}, J=7.8,1.5 \mathrm{~Hz}, \mathrm{Ar}) ;{ }^{13} \mathrm{C}-\mathrm{NMR}\left(125 \mathrm{MHz}, \mathrm{CDCl}_{3}\right) \delta: 23.3$, 24.3, 47.5, 49.0, 124.2, 126.6, 127.5, 129.3, 129.9, 132.0, 151.0, 155.5; HRMS (FAB) $m / z$ calcd for $\mathrm{C}_{12} \mathrm{H}_{14} \mathrm{~N}_{3} \mathrm{~S}[\mathrm{M}+\mathrm{H}]^{+}$232.0908; found: 232.0906 .

\subsubsection{Synthesis of 9-Bromo-2H-spiro(benzo[e]pyrimido [1,2-c][1,3]thiazine-3,1'-cyclohexan)-6(4H)- imine (49)}

Cyclohexane-1,1-dicarbonitrile (42). To a solution of malononitrile (660.6 mg, $10.0 \mathrm{mmol})$ in DMF $(25.0 \mathrm{~mL})$ was added DBU $(2.99 \mathrm{~mL}, 20.0 \mathrm{mmol})$. After being stirred at $50{ }^{\circ} \mathrm{C}$ for $2 \mathrm{~h}$, a solution of 1,5-dibromopentane $38(1.35 \mathrm{~mL}$, $10.0 \mathrm{mmol})$ in DMF $(10.0 \mathrm{~mL})$ was added to the reaction mixture. After being stirred at the same temperature for additional $5 \mathrm{~h}$, EtOAc was added. The mixture was washed with $5 \%$ aq. $\mathrm{NaHCO}_{3}$, and dried over $\mathrm{MgSO}_{4}$. After concentration, the residue was purified by flash chromatography over silica gel with $n$-hexaneEtOAc (3:1). The resulting solid was recrystalized from $\mathrm{CHCl}_{3}-n$-hexane to give the title compound 42 as colorless crystals $(801.8 \mathrm{mg}, 60 \%): \mathrm{mp} 62{ }^{\circ} \mathrm{C}$, IR (neat) $\mathrm{cm}^{-1}: 2254(\mathrm{C} \equiv \mathrm{N}) ;{ }^{1} \mathrm{H}-\mathrm{NMR}\left(400 \mathrm{MHz}, \mathrm{CDCl}_{3}\right) \delta: 1.51-1.57\left(\mathrm{~m}, 2 \mathrm{H}, \mathrm{CH}_{2}\right)$, 1.73-1.78(m, 4H, $\left.2 \times \mathrm{CH}_{2}\right), 2.13\left(\mathrm{t}, J=5.9 \mathrm{~Hz}, 4 \mathrm{H}, 2 \times \mathrm{CH}_{2}\right) ;{ }^{13} \mathrm{C}-\mathrm{NMR}$ $\left(100 \mathrm{MHz}, \mathrm{CDCl}_{3}\right) \delta: 21.6(2 \mathrm{C}), 23.9,32.4,34.6$ (2C), 115.9 (2C); MS (FAB) $\mathrm{m} / z$ (\%): $135\left(\mathrm{MH}^{+}, 100\right)$.

3-(4-Bromo-2-fluorophenyl)-2,4-diazaspiro[5.5] undec-2-ene (45). To a solution of 42 (134.2 mg, $1.0 \mathrm{mmol})$ in THF $(2.5 \mathrm{~mL})$ was added $\mathrm{BH}_{3}$-THF in THF (5.0 mL, $5.0 \mathrm{mmol}, 1.0 \mathrm{M}$ ) at $0{ }^{\circ} \mathrm{C}$ under an $\mathrm{Ar}$ atmosphere. The mixture was warmed to rt. After being stirred at $65{ }^{\circ} \mathrm{C}$ for $5 \mathrm{~h}$, the reaction mixture was cooled to $0{ }^{\circ} \mathrm{C}$, and was added $1 \mathrm{~N} \mathrm{HCl}$. After being stirred at $\mathrm{rt}$ for $1 \mathrm{~h}$, the mixture was basified with $2 \mathrm{~N} \mathrm{NaOH}$. The whole was extracted with $\mathrm{CHCl}_{3}$ and dried over $\mathrm{MgSO}_{4}$. After concentration, the residue was dissolved in $t$ - $\mathrm{BuOH}$ (10.0 mL), and 4-bromo-2-fluorobenzaldehyde (203.0 mg, $1.0 \mathrm{mmol})$ was added. 
After being stirred at $70{ }^{\circ} \mathrm{C}$ for $30 \mathrm{~min}, \mathrm{~K}_{2} \mathrm{CO}_{3}(414.6 \mathrm{mg}, 3.0 \mathrm{mmol})$ and $\mathrm{I}_{2}$ (317.3 $\mathrm{mg}, 1.25 \mathrm{mmol}$ ) were added. After being stirred at same temperature for $3 \mathrm{~h}$, the reaction mixture was quenched with sat. $\mathrm{Na}_{2} \mathrm{SO}_{3}$ until the iodine color almost disappeared. The reaction mixture was basified with $2 \mathrm{~N} \mathrm{NaOH}$. The whole was extracted with $\mathrm{CHCl}_{3}$, and dried over $\mathrm{MgSO}_{4}$. After concentration, the residue was purified by flash chromatography over aluminum oxide with EtOAc-MeOH (1:0 to 95:5) gave the title compound $\mathbf{4 5}$ as colorless solid (200.1 mg, $62 \%): \mathrm{mp}$ 204-205 ${ }^{\circ} \mathrm{C}$ (from $\mathrm{CHCl}_{3}-n$-hexane), IR (neat) $\mathrm{cm}^{-1}$ : $1626(\mathrm{C}=\mathrm{N}) ;{ }^{1} \mathrm{H}-\mathrm{NMR}$ $\left(500 \mathrm{MHz}, \mathrm{CDCl}_{3}\right) \delta: 1.35-1.37\left(\mathrm{~m}, 4 \mathrm{H}, 2 \times \mathrm{CH}_{2}\right), 1.47-1.49\left(\mathrm{~m}, 6 \mathrm{H}, 3 \times \mathrm{CH}_{2}\right)$, $3.20\left(\mathrm{~s}, 4 \mathrm{H}, 2 \times \mathrm{CH}_{2}\right), 5.07(\mathrm{~s}, 1 \mathrm{H}, \mathrm{NH}), 7.23(\mathrm{dd}, J=11.2,2.0 \mathrm{~Hz}, 1 \mathrm{H}, \mathrm{Ar}), 7.28$ $(\mathrm{dd}, J=8.3,2.0 \mathrm{~Hz}, 1 \mathrm{H}, \mathrm{Ar}), 7.69(\mathrm{t}, J=8.3 \mathrm{~Hz}, 1 \mathrm{H}, \mathrm{Ar}) ;{ }^{13} \mathrm{C}-\mathrm{NMR}(125 \mathrm{MHz}$, $\left.\mathrm{CDCl}_{3}\right) \delta: 21.7(2 \mathrm{C}), 26.5,28.8(2 \mathrm{C}), 33.5,52.2(2 \mathrm{C}), 119.4(\mathrm{~d}, J=27.6 \mathrm{~Hz})$, $123.1(\mathrm{~d}, J=12.0 \mathrm{~Hz}), 123.5(\mathrm{~d}, J=10.8 \mathrm{~Hz}), 127.7(\mathrm{~d}, J=3.6 \mathrm{~Hz}), 131.8(\mathrm{~d}$, $J=3.6 \mathrm{~Hz}), 149.9,159.8(\mathrm{~d}, J=251.9 \mathrm{~Hz}) ;{ }^{19} \mathrm{~F}-\mathrm{NMR}\left(500 \mathrm{MHz}, \mathrm{CDCl}_{3}\right) \delta$ : -114.6; HRMS (FAB): $m / z$ calcd for $\mathrm{C}_{15} \mathrm{H}_{19} \mathrm{BrFN}_{2}[\mathrm{M}+\mathrm{H}]^{+} 325.0716$; found: 325.0724 .

9-Bromo- $N$-(tert-butyl)-2H-spiro(benzo[e]pyrimido[1,2-c][1,3]thiazine-3,1'cyclohexan)-6(4H)-imine (48). To a mixture of compound 45 (164.5 mg, $0.51 \mathrm{mmol}$ ) and $\mathrm{NaH}(40.8 \mathrm{mg}, 1.02 \mathrm{mmol} ; 60 \%$ oil suspension) in DMF $(3.3 \mathrm{~mL})$ was added $t$-BuNCS $(129.4 \mu \mathrm{L}, 1.02 \mathrm{mmol})$ under an Ar atmosphere. After being stirred at $\mathrm{rt}$ overnight, the reaction mixture was warmed at $60{ }^{\circ} \mathrm{C}$. After being stirred at this temperature for $1 \mathrm{~h}$, EtOAc was added. The resulting solution was washed with sat. $\mathrm{NaHCO}_{3}$, brine, and dried over $\mathrm{MgSO}_{4}$. After concentration, the residue was purified by flash chromatography over aluminum oxide with $n$-hexane-EtOAc (1:0 to 9:1) gave the title compound $\mathbf{4 8}$ as colorless solid (180.1 mg, $84 \%$ ): $\mathrm{mp} 118-119{ }^{\circ} \mathrm{C}$ (from $n$-hexane); IR (neat) $\mathrm{cm}^{-1}: 1578$ $(\mathrm{C}=\mathrm{N}) ;{ }^{1} \mathrm{H}-\mathrm{NMR}\left(500 \mathrm{MHz}, \mathrm{CDCl}_{3}\right) \delta: 1.32-1.37\left(\mathrm{~m}, 4 \mathrm{H}, 2 \times \mathrm{CH}_{2}\right), 1.38(\mathrm{~s}, 9 \mathrm{H}$, $\left.3 \times \mathrm{CH}_{3}\right), 1.43-1.52\left(\mathrm{~m}, 6 \mathrm{H}, 3 \times \mathrm{CH}_{2}\right), 3.38\left(\mathrm{~s}, 2 \mathrm{H}, \mathrm{CH}_{2}\right), 3.71\left(\mathrm{~s}, 2 \mathrm{H}, \mathrm{CH}_{2}\right)$, 7.26-7.31 (m, 2H, Ar), 8.04 (d, $J=8.6 \mathrm{~Hz}, 1 \mathrm{H}, \mathrm{Ar}) ;{ }^{13} \mathrm{C}-\mathrm{NMR}(125 \mathrm{MHz}$, $\left.\mathrm{CDCl}_{3}\right) \delta: 21.8(2 \mathrm{C}), 26.5,29.9$ (3C), 31.2, 33.4 (2C), 52.5, 54.2, 55.9, 124.3, $126.5,126.8,129.1,130.0,130.9,137.6,146.2$; HRMS (FAB): $\mathrm{m} / \mathrm{z}$ calcd for $\mathrm{C}_{20} \mathrm{H}_{27} \mathrm{BrN}_{3} \mathrm{~S}[\mathrm{M}+\mathrm{H}]^{+} 420.1109$; found: 420.1117 .

Compound 49. Using the general procedure as described for 25a, 48 (124.7 $\mathrm{mg}, 0.3 \mathrm{mmol}$ ) was allowed to react under reflux for $2.5 \mathrm{~h}$ with TFA (3.0 mL) and MS4A (0.45 g). Purification by flash chromatography over aluminum oxide with $n$-hexane-EtOAc (7:3) gave the title compound 49 as pale yellow solid (89.8 mg, $82 \%$ ): $\mathrm{mp} 130{ }^{\circ} \mathrm{C}$ (from $\mathrm{CHCl}_{3}-n$-hexane); IR (neat) $\mathrm{cm}^{-1}: 1626$ $(\mathrm{C}=\mathrm{N}), 1572(\mathrm{C}=\mathrm{N}) ;{ }^{1} \mathrm{H}-\mathrm{NMR}\left(400 \mathrm{MHz}, \mathrm{CDCl}_{3}\right) \delta: 1.37-1.55(\mathrm{~m}, 10 \mathrm{H}$, $\left.5 \times \mathrm{CH}_{2}\right), 3.46\left(\mathrm{~s}, 2 \mathrm{H}, \mathrm{CH}_{2}\right), 3.82\left(\mathrm{~s}, 2 \mathrm{H}, \mathrm{CH}_{2}\right), 7.21(\mathrm{~d}, J=2.0 \mathrm{~Hz}, 1 \mathrm{H}, \mathrm{Ar}), 7.24$ $(\mathrm{s}, 1 \mathrm{H}, \mathrm{NH}), 7.33(\mathrm{dd}, J=8.8,2.0 \mathrm{~Hz}, 1 \mathrm{H}, \mathrm{Ar}), 8.10(\mathrm{~d}, J=8.8 \mathrm{~Hz}, 1 \mathrm{H}, \mathrm{Ar}) ;{ }^{13} \mathrm{C}-$ $\mathrm{NMR}\left(100 \mathrm{MHz}, \mathrm{CDCl}_{3}\right) \delta: 21.6$ (2C), 26.3, 30.6, 33.3 (2C), 51.4, 55.4, 125.0, $125.3,125.9,129.5,130.5,130.7,144.8,152.7$; HRMS (FAB): $m / z$ calcd for $\mathrm{C}_{16} \mathrm{H}_{19} \mathrm{BrN}_{3} \mathrm{~S}[\mathrm{M}+\mathrm{H}]^{+}$364.0483; found: 364.0485 . 


\subsubsection{Synthesis of 9-Bromo-2', $3^{\prime}, 5^{\prime}, 6^{\prime}$-tetrahydro- $2 \mathrm{H}$ - spiro(benzo[e]pyrimido[1,2-c][1,3]thiazine- 3,4'-pyran)-6(4H)-imine (51)}

Dihydro-2H-pyran-4,4(3H)-dicarbonitrile (43). To a solution of malononitrile $(660.6 \mathrm{mg}, 10.0 \mathrm{mmol})$ in DMF $(25.0 \mathrm{~mL})$ was added DBU $(2.99 \mathrm{~mL}$, $20.0 \mathrm{mmol}$ ). After stirring at $50{ }^{\circ} \mathrm{C}$ for $2 \mathrm{~h}$, the reaction mixture was added a solution of bis(2-chloroethyl)ether $39(1.18 \mathrm{~mL}, 10.0 \mathrm{mmol})$ in DMF (10.0 mL). After being stirred at same temperature for $5 \mathrm{~h}$, EtOAc was added. The mixture was washed with $5 \%$ aq. $\mathrm{NaHCO}_{3}$, and dried over $\mathrm{MgSO}_{4}$. The filtrate was concentrated. The residue was purified by flash chromatography over silica gel with $n$-hexane-EtOAc (3:1). The resulting solid was recrystalized from $\mathrm{CHCl}_{3}-n$ hexane to give the title compound $\mathbf{4 3}$ as colorless crystals (112.2 mg, $8 \%): \mathrm{mp}$ $96{ }^{\circ} \mathrm{C}$, IR (neat) $\mathrm{cm}^{-1}: 2253(\mathrm{C} \equiv \mathrm{N}) ;{ }^{1} \mathrm{H}-\mathrm{NMR}\left(400 \mathrm{MHz}, \mathrm{CDCl}_{3}\right) \delta: 2.24$ (t, $\left.J=5.2 \mathrm{~Hz}, 4 \mathrm{H}, 2 \times \mathrm{CH}_{2}\right), 3.87\left(\mathrm{t}, J=5.2 \mathrm{~Hz}, 4 \mathrm{H}, 2 \times \mathrm{CH}_{2}\right) ;{ }^{13} \mathrm{C}-\mathrm{NMR}$ $\left(100 \mathrm{MHz} \mathrm{CDCl}_{3}\right) \delta: 30.2,33.8$ (2C), 63.0 (2C), 114.9 (2C); MS (FAB) $\mathrm{m} / \mathrm{z}(\%)$ : $137\left(\mathrm{MH}^{+}, 100\right)$.

3-(4-Bromo-2-fluorophenyl)-9-oxa-2,4-diazaspiro[5.5]undec-2-ene

(46).

Using the general procedure as described for $\mathbf{4 5}, \mathbf{4 3}(84.1 \mathrm{mg}, 0.62 \mathrm{mmol})$ was allowed to react. Purification by flash chromatography over aluminum oxide with EtOAc-MeOH (1:0 to 95:5) gave the title compound 46 as colorless solid (21.4 mg, $11 \%$ ): $\mathrm{mp} 200-201{ }^{\circ} \mathrm{C}$ (from $\mathrm{CHCl}_{3}-n$-hexane); IR (neat) $\mathrm{cm}^{-1}: 1626$ $(\mathrm{C}=\mathrm{N}) ;{ }^{1} \mathrm{H}-\mathrm{NMR}\left(500 \mathrm{MHz}, \mathrm{CDCl}_{3}\right) \delta: 1.52\left(\mathrm{t}, J=5.4 \mathrm{~Hz}, 4 \mathrm{H}, 2 \times \mathrm{CH}_{2}\right), 3.31$ $\left(\mathrm{s}, 4 \mathrm{H}, 2 \times \mathrm{CH}_{2}\right), 3.72\left(\mathrm{t}, J=5.4 \mathrm{~Hz}, 4 \mathrm{H}, 2 \times \mathrm{CH}_{2}\right), 4.03($ br s, $1 \mathrm{H}, \mathrm{NH}), 7.25$ $(\mathrm{dd}, J=11.7,2.0 \mathrm{~Hz}, 1 \mathrm{H}, \mathrm{Ar}), 7.31(\mathrm{dd}, J=8.6,2.0 \mathrm{~Hz}, 1 \mathrm{H}, \mathrm{Ar}), 7.70(\mathrm{t}$, $J=8.6 \mathrm{~Hz}, 1 \mathrm{H}, \mathrm{Ar}) ;{ }^{13} \mathrm{C}-\mathrm{NMR}\left(125 \mathrm{MHz}, \mathrm{CDCl}_{3}\right) \delta: 27.0,33.3$ (2C), $51.4(2 \mathrm{C})$, $63.7(2 \mathrm{C}), 119.5(\mathrm{~d}, J=27.6 \mathrm{~Hz}), 122.5(\mathrm{~d}, J=13.2 \mathrm{~Hz}), 124.0(\mathrm{~d}, J=9.6 \mathrm{~Hz})$, $127.9(\mathrm{~d}, J=3.6 \mathrm{~Hz}), 131.7(\mathrm{~d}, J=3.6 \mathrm{~Hz}), 150.4,159.7(\mathrm{~d}, J=251.9 \mathrm{~Hz})$; HRMS (FAB): $m / z$ calcd for $\mathrm{C}_{14} \mathrm{H}_{17} \mathrm{BrFN}_{2} \mathrm{O}[\mathrm{M}+\mathrm{H}]^{+}$327.0508; found: 327.0512.

9-Bromo- $N$-(tert-butyl)-2' $, 3^{\prime}, 5^{\prime}, 6^{\prime}$-tetrahydro-2H-spiro(benzo[e]pyrimido[1, $2-c][1,3]$ thiazine-3,4'-pyran)-6(4H)-imine (50). Using the general procedure as described for 48, $46(21.4 \mathrm{mg}, 0.065 \mathrm{mmol})$ was allowed to react at rt overnight. Purification by flash chromatography over aluminum oxide with $n$-hexane-EtOAc (1:0 to 9:1) gave the title compound 50 as colorless solid (21.5 mg, $78 \%$ ): $\mathrm{mp}$ 148-149 ${ }^{\circ} \mathrm{C}$ (from $n$-hexane); IR (neat) $\mathrm{cm}^{-1}$ : $1578(\mathrm{C}=\mathrm{N}) ;{ }^{1} \mathrm{H}-\mathrm{NMR}(400 \mathrm{MHz}$, $\left.\mathrm{CDCl}_{3}\right) \delta: 1.38\left(\mathrm{~s}, 9 \mathrm{H}, 3 \times \mathrm{CH}_{3}\right), 1.48-1.53\left(\mathrm{~m}, 4 \mathrm{H}, 2 \times \mathrm{CH}_{2}\right), 3.48\left(\mathrm{~s}, 2 \mathrm{H}, \mathrm{CH}_{2}\right)$, 3.71-3.74 (m, 4H, $\left.2 \times \mathrm{CH}_{2}\right), 3.84\left(\mathrm{~s}, 2 \mathrm{H}, \mathrm{CH}_{2}\right), 7.29-7.33(\mathrm{~m}, 1 \mathrm{H}, \mathrm{Ar}), 7.37$ (d, $J=4.1 \mathrm{~Hz}, 1 \mathrm{H}, \mathrm{Ar}), 8.05(\mathrm{~d}, J=8.5 \mathrm{~Hz}, 1 \mathrm{H}, \mathrm{Ar}) ;{ }^{13} \mathrm{C}-\mathrm{NMR}\left(125 \mathrm{MHz}, \mathrm{CDCl}_{3}-\right.$ $\left.\mathrm{CD}_{3} \mathrm{OD}\right) \delta$ : 29.4, 29.9 (3C), 33.1 (2C), 51.4, 54.3, 55.5, 63.8 (2C), 124.6, 126.1, $126.9,129.3,130.0,130.9,137.6,146.4$; HRMS (FAB): $\mathrm{m} / \mathrm{z}$ calcd for $\mathrm{C}_{19} \mathrm{H}_{25} \mathrm{BrN}_{3} \mathrm{OS}[\mathrm{M}+\mathrm{H}]^{+}$422.0902; found:422.0898.

Compound 51. Using the general procedure as described for 25a, 50 (21.4 mg, $0.065 \mathrm{mmol})$ was allowed to react under reflux for $2.5 \mathrm{~h}$ with TFA $(1.0 \mathrm{~mL})$ and 
MS4Å (150 mg). Purification by flash chromatography over aluminum oxide with $n$-hexane-EtOAc (7:3) gave the title compound $\mathbf{5 1}$ as colorless solid (12.3 $\mathrm{mg}$, $66 \%$ ): mp 212-214 ${ }^{\circ} \mathrm{C}$ (from $\mathrm{CHCl}_{3}-n$-hexane); IR (neat) $\mathrm{cm}^{-1}: 1626(\mathrm{C}=\mathrm{N})$, $1573(\mathrm{C}=\mathrm{N}) ;{ }^{1} \mathrm{H}-\mathrm{NMR}\left(400 \mathrm{MHz}, \mathrm{CDCl}_{3}\right) \delta: 1.54\left(\mathrm{t}, J=5.4 \mathrm{~Hz}, 4 \mathrm{H}, 2 \times \mathrm{CH}_{2}\right)$, $3.56\left(\mathrm{~s}, 2 \mathrm{H}, \mathrm{CH}_{2}\right), 3.74\left(\mathrm{t}, J=5.4 \mathrm{~Hz}, 4 \mathrm{H}, 2 \times \mathrm{CH}_{2}\right), 3.93\left(\mathrm{~s}, 2 \mathrm{H}, \mathrm{CH}_{2}\right), 7.22(\mathrm{~d}$, $J=2.0 \mathrm{~Hz}, 1 \mathrm{H}, \mathrm{Ar}), 7.31$ (br s, $1 \mathrm{H}, \mathrm{NH}), 7.34$ (dd, $J=8.8,2.0 \mathrm{~Hz}, 1 \mathrm{H}, \mathrm{Ar}), 8.10$ $(\mathrm{d}, J=8.8 \mathrm{~Hz}, 1 \mathrm{H}, \mathrm{Ar}) ;{ }^{13} \mathrm{C}-\mathrm{NMR}\left(100 \mathrm{MHz}, \mathrm{CDCl}_{3}-\mathrm{CD}_{3} \mathrm{OD}\right) \delta: 28.6,32.9$ (2C), 50.7, 54.6, 63.4 (2C), 124.7, 125.3, 125.9, 129.6, 130.3, 130.5, 145.4, 152.9; HRMS (FAB): $m / z$ calcd for $\mathrm{C}_{15} \mathrm{H}_{17} \mathrm{BrN}_{3} \mathrm{OS}[\mathrm{M}+\mathrm{H}]^{+} 366.0276$; found: 366.0280 .

\subsubsection{Synthesis of 9-Bromo-1'-(4-methoxybenzyl)-2H- spiro(benzo[e]pyrimido[1,2-c][1,3]thiazine-3,4'- piperidin)-6(4H)-imine (53a)}

Bis(2-chloroethyl)- $\mathrm{N}$-(4-methoxybenzyl)amine (41). To a suspension of bis(2chloroethyl)amine hydrochloride $40(8.92 \mathrm{~g}, 50.0 \mathrm{mmol})$ in $\mathrm{CH}_{2} \mathrm{Cl}_{2}(300 \mathrm{~mL})$ were added $\mathrm{Et}_{3} \mathrm{~N}(2.89 \mathrm{~mL}, 100.0 \mathrm{mmol})$ and 4-methoxybenzoyl chloride $(6.77 \mathrm{~mL}, 50.0 \mathrm{mmol})$. After being stirred at $\mathrm{rt}$ for $2 \mathrm{~h}$, the reaction mixture was washed with $1 \mathrm{~N} \mathrm{HCl}$, sat. $\mathrm{NaHCO}_{3}$, brine, and dried over $\mathrm{MgSO}_{4}$. After concentration, the residue was dissolved in anhydrous $\mathrm{Et}_{2} \mathrm{O}(250 \mathrm{~mL})$, and $\mathrm{LiAlH}_{4}$ $(2.1 \mathrm{~g}, 55.0 \mathrm{mmol})$ was slowly added to the mixture at $0{ }^{\circ} \mathrm{C}$ under an Ar atmosphere. After being stirred at rt overnight, the reaction mixture was quenched by the addition of water, $2 \mathrm{~N} \mathrm{NaOH}$, and water. The mixture was dried over $\mathrm{MgSO}_{4}$. After concentration, the residue was purified by flash chromatography over silica gel with $n$-hexane-EtOAc (19:1) to give the title compound $\mathbf{4 1}$ as colorless oil (9.88 g, $75 \%):{ }^{1} \mathrm{H}-\mathrm{NMR}\left(400 \mathrm{MHz}, \mathrm{CDCl}_{3}\right) \delta: 2.90 \quad(\mathrm{t}, J=7.1 \mathrm{~Hz}, \quad 4 \mathrm{H}$, $\left.2 \times \mathrm{CH}_{2}\right), 3.48\left(\mathrm{t}, J=7.1 \mathrm{~Hz}, 4 \mathrm{H}, 2 \times \mathrm{CH}_{2}\right), 3.67\left(\mathrm{~s}, 2 \mathrm{H}, \mathrm{CH}_{2}\right), 3.80(\mathrm{~s}, 3 \mathrm{H}$, $\left.\mathrm{CH}_{3}\right), 6.86(\mathrm{~d}, J=8.5 \mathrm{~Hz}, 2 \mathrm{H}, \mathrm{Ar}), 7.24(\mathrm{~d}, J=8.5 \mathrm{~Hz}, 2 \mathrm{H}, \mathrm{Ar}) ;{ }^{13} \mathrm{C}-\mathrm{NMR}$ $\left(100 \mathrm{MHz} \mathrm{CDCl}_{3}\right) \delta: 42.0$ (2C), 55.2, 56.2 (2C), 58.6, 113.8 (2C), 129.7 (2C), 130.7, 158.9; MS (FAB) $m / z(\%): 262\left(\mathrm{MH}^{+}, 100\right)$.

1-(4-Methoxybenzyl)piperidine-4,4-dicarbonitrile (44). To a solution of malononitrile $(2.49 \mathrm{~g}, 37.7 \mathrm{mmol})$ in DMF $(94.3 \mathrm{~mL})$ was added $\mathrm{K}_{2} \mathrm{CO}_{3}$ (5.73 mg, $41.5 \mathrm{mmol})$. After being stirred at $65^{\circ} \mathrm{C}$ for $2 \mathrm{~h}$, a solution of 41 $(9.88 \mathrm{mg}, 37.7 \mathrm{mmol})$ in DMF $(37.7 \mathrm{~mL})$ was added. After being stirred at same temperature for $5 \mathrm{~h}$, EtOAc was added. The mixture was washed with $5 \%$ aq. $\mathrm{NaHCO}_{3}$, and dried over $\mathrm{MgSO}_{4}$. After concentration, the residue was purified by flash chromatography over silica gel with $n$-hexane-EtOAc $(2: 1)$ to give the title compound 44 as yellow oil $(8.13 \mathrm{~g}, 85 \%)$ : IR (neat) $\mathrm{cm}^{-1}: 2248(\mathrm{C} \equiv \mathrm{N}) ;{ }^{1} \mathrm{H}$ $\mathrm{NMR}\left(400 \mathrm{MHz}, \mathrm{CDCl}_{3}\right) \delta: 2.22\left(\mathrm{t}, J=5.4 \mathrm{~Hz}, 4 \mathrm{H}, 2 \times \mathrm{CH}_{2}\right), 2.61$ (br s, $4 \mathrm{H}$, $\left.2 \times \mathrm{CH}_{2}\right), 3.48\left(\mathrm{~s}, 2 \mathrm{H}, \mathrm{CH}_{2}\right), 3.80\left(\mathrm{~s}, 3 \mathrm{H}, \mathrm{CH}_{2}\right), 6.86(\mathrm{~d}, J=8.5 \mathrm{~Hz}, 2 \mathrm{H}, \mathrm{Ar}), 7.19$ $(\mathrm{d}, J=8.8 \mathrm{~Hz}, 2 \mathrm{H}, \mathrm{Ar}) ;{ }^{13} \mathrm{C}-\mathrm{NMR}\left(100 \mathrm{MHz}, \mathrm{CDCl}_{3}\right) \delta: 31.1,34.1(2 \mathrm{C}), 48.5$ 
(2C), 55.2, 61.9, 113.8 (2C), 115.4 (2C), 129.2, 130.1 (2C), 159.0; HRMS (FAB): $m / z$ calcd for $\mathrm{C}_{15} \mathrm{H}_{18} \mathrm{~N}_{3} \mathrm{O}[\mathrm{M}+\mathrm{H}]^{+} 256.1450$; found: 256.1454 .

3-(4-Bromo-2-fluorophenyl)-9-(4-methoxybenzyl)-2,4,9-triazaspiro[5.5] undec-2-ene (47). Using the general procedure as described for 45, 44 (4.05 g, $15.9 \mathrm{mmol}$ ) was allowed to react. Purification by flash chromatography over aluminum oxide with EtOAc-MeOH (1:0 to 95:5) to give the title compound 47 as colorless solid (752.6 mg, $11 \%$ ): $\mathrm{mp} 179-181{ }^{\circ} \mathrm{C}$ (from $\mathrm{CHCl}_{3}-n$-hexane), IR (neat) $\mathrm{cm}^{-1}: 1630(\mathrm{C}=\mathrm{N}) ;{ }^{1} \mathrm{H}-\mathrm{NMR}\left(500 \mathrm{MHz}, \mathrm{CDCl}_{3}\right) \delta: 1.45(\mathrm{t}, J=5.4 \mathrm{~Hz}, 4 \mathrm{H}$, $\left.2 \times \mathrm{CH}_{2}\right), 2.35\left(\mathrm{t}, J=5.4 \mathrm{~Hz}, 4 \mathrm{H}, 2 \times \mathrm{CH}_{2}\right), 3.16\left(\mathrm{~s}, 4 \mathrm{H}, 2 \times \mathrm{CH}_{2}\right), 3.40(\mathrm{~s}, 2 \mathrm{H}$, $\left.\mathrm{CH}_{2}\right), 3.73$ (s, 3H, $\left.\mathrm{CH}_{3}\right), 4.63$ (s, 1H, NH), 6.78 (d, $\left.J=8.6 \mathrm{~Hz}, 2 \mathrm{H}, \mathrm{Ar}\right), 7.14-7.23$ (m, 4H, Ar), $7.62(\mathrm{t}, J=8.3 \mathrm{~Hz}, 1 \mathrm{H}, \mathrm{Ar}) ;{ }^{13} \mathrm{C}-\mathrm{NMR}\left(100 \mathrm{MHz}, \mathrm{CDCl}_{3}\right) \delta: 27.3$, 32.8 (2C), 49.1 (2C), 51.4 (2C), 55.2, 62.7, $113.5(2 \mathrm{C}), 119.4$ (d, $J=27.3 \mathrm{~Hz})$, $122.7(\mathrm{~d}, J=12.4 \mathrm{~Hz}), 123.7(\mathrm{~d}, J=9.9 \mathrm{~Hz}), 127.8(\mathrm{~d}, J=3.3 \mathrm{~Hz}), 130.2$, $130.3(2 \mathrm{C}), 131.7$ (d, $J=4.1 \mathrm{~Hz}), 150.3$ (d, $J=1.7 \mathrm{~Hz}), 158.6,159.7$ (d, $J=251.6 \mathrm{~Hz}) ;{ }^{19} \mathrm{~F}-\mathrm{NMR}\left(500 \mathrm{MHz}, \mathrm{CDCl}_{3}\right) \delta:-114.6$; HRMS (FAB): $m / z$ calcd for $\mathrm{C}_{22} \mathrm{H}_{26} \mathrm{BrFN}_{3} \mathrm{O}[\mathrm{M}+\mathrm{H}]^{+} 446.1243$; found: 446.1237 .

9-Bromo- $N$-(tert-butyl)-1' -(4-methoxybenzyl)-2H-spiro(benzo[e]pyrimido[1, 2-c][1,3]thiazine-3,4' -piperidin)-6(4H)-imine (52a). Using the general procedure as described for 48, $47(2.0 \mathrm{~g}, 4.48 \mathrm{mmol})$ was allowed to react at rt overnight. Purification by flash chromatography over aluminum oxide with $n$-hexane-EtOAc (1:0 to 9:1) to give the title compound 52a as colorless solid (2.28 g, $94 \%): \mathrm{mp}$ 89-91 ${ }^{\circ} \mathrm{C}$ (from $\mathrm{CHCl}_{3}-n$-hexane); IR (neat) $\mathrm{cm}^{-1}$ : $1577 \quad(\mathrm{C}=\mathrm{N}) ;{ }^{1} \mathrm{H}-\mathrm{NMR}$ $\left(500 \mathrm{MHz}, \mathrm{CDCl}_{3}\right) \delta: 1.37\left(\mathrm{~s}, 9 \mathrm{H}, 3 \times \mathrm{CH}_{3}\right), 1.49-1.52\left(\mathrm{~m}, 4 \mathrm{H}, 2 \times \mathrm{CH}_{2}\right)$, 2.40-2.46 (m, 4H, $\left.2 \times \mathrm{CH}_{2}\right), 3.41\left(\mathrm{~s}, 2 \mathrm{H}, \mathrm{CH}_{2}\right), 3.47\left(\mathrm{~s}, 2 \mathrm{H}, \mathrm{CH}_{2}\right), 3.75(\mathrm{~s}, 2 \mathrm{H}$, $\left.\mathrm{CH}_{2}\right), 3.80\left(\mathrm{~s}, 3 \mathrm{H}, \mathrm{CH}_{3}\right), 6.85(\mathrm{~d}, J=8.6 \mathrm{~Hz}, 2 \mathrm{H}, \mathrm{Ar}), 7.22(\mathrm{~d}, J=8.6 \mathrm{~Hz}, 2 \mathrm{H}$, Ar), 7.28-7.31 (m, 2H, Ar), 8.03 (d, $J=8.6 \mathrm{~Hz}, 1 \mathrm{H}, \mathrm{Ar}) ;{ }^{13} \mathrm{C}-\mathrm{NMR}(100 \mathrm{MHz}$, $\left.\mathrm{CDCl}_{3}\right) \delta: 29.7,29.9$ (3C), $32.6(2 \mathrm{C}), 49.2(2 \mathrm{C}), 51.6,54.3,55.2,55.5,62.7,113.6$ (2C), 124.5, 126.3, 126.8, 129.2, 130.0, 130.1, 130.4 (2C), 130.9, 137.5, 146.3, 158.7; HRMS (FAB): $m / z$ calcd for $\mathrm{C}_{27} \mathrm{H}_{34} \mathrm{BrN}_{4} \mathrm{OS}[\mathrm{M}+\mathrm{H}]^{+}$541.1637; found: 541.1633 .

Compound 53a. Using the general procedure as described for 25a, compound 52a $(448.1 \mathrm{mg}, 0.83 \mathrm{mmol})$ was allowed to react for $2 \mathrm{~h}$ with TFA $(10.0 \mathrm{~mL})$ and MS4A (1.50 g). Purification by flash chromatography over aluminum oxide with $n$-hexane-EtOAc (7:3) gave the title compound 53a as colorless solid (288.8 $\mathrm{mg}$, $72 \%$ ): mp $160-162{ }^{\circ} \mathrm{C}$ (from $\mathrm{CHCl}_{3}-n$-hexane); IR (neat) $\mathrm{cm}^{-1}: 1626(\mathrm{C}=\mathrm{N})$, $1573(\mathrm{C}=\mathrm{N}) ;{ }^{1} \mathrm{H}-\mathrm{NMR}\left(400 \mathrm{MHz}, \mathrm{CDCl}_{3}\right) \delta: 1.54\left(\mathrm{t}, J=5.5 \mathrm{~Hz}, 4 \mathrm{H}, 2 \times \mathrm{CH}_{2}\right)$, 2.39-2.51 (m, 4H, $\left.2 \times \mathrm{CH}_{2}\right), 3.46\left(\mathrm{~s}, 2 \mathrm{H}, \mathrm{CH}_{2}\right), 3.48\left(\mathrm{~s}, 2 \mathrm{H}, \mathrm{CH}_{2}\right), 3.79(\mathrm{~s}, 3 \mathrm{H}$, $\left.\mathrm{CH}_{3}\right), 3.86$ (s, 2H, $\mathrm{CH}_{2}$ ), 6.84 (d, J = 8.8 Hz, 2H, Ar), 7.20-7.22 (m, 3H, Ar), 7.28 $(\mathrm{s}, 1 \mathrm{H}, \mathrm{NH}), 7.32(\mathrm{dd}, J=8.8,2.0 \mathrm{~Hz}, 1 \mathrm{H}, \mathrm{Ar}), 8.08(\mathrm{~d}, J=8.8 \mathrm{~Hz}, 1 \mathrm{H}, \mathrm{Ar}) ;{ }^{13} \mathrm{C}-$ NMR (100 MHz, $\left.\mathrm{CDCl}_{3}\right) \delta:$ 29.2, 32.6 (2C), 49.1 (2C), 50.8, 55.1, 55.2, 62.6, 113.6 (2C), 125.1, 125.1, 125.9, 129.5, 130.3 (2C), 130.3, 130.4, 130.7, 145.0, 152.6, 158.7; HRMS (FAB): $m / z$ calcd for $\mathrm{C}_{23} \mathrm{H}_{26} \mathrm{BrN}_{4} \mathrm{OS}[\mathrm{M}+\mathrm{H}]^{+} 485.1011$; found: 485.1010 . 


\subsubsection{Synthesis of 9-Bromo-1'-(methoxycarbonyl)-2H- spiro(benzo[e]pyrimido[1,2-c][1,3]thiazine-3,4'- piperidin)-6(4H)-imine (53b)}

9-Bromo- $N$-(tert-butyl)-1' -(methoxycarbonyl)- $2 H$-spiro(benzo[e]pyrimido[1, 2-c $][1,3]$ thiazine-3, $4^{\prime}$-piperidin)-6(4H)-imine (52b). To the solution of 9-bromo$N$-(tert-butyl)-1'-(4-methoxybenzyl)-2H-spiro(benzo[e]pyrimido[1,2-c][1,3]thiazine-3,4'-piperidin)-6(4H)-imine $\quad \mathbf{5 2 a} \quad(40.6 \mathrm{mg}, \quad 0.075 \mathrm{mmol})$ in $\mathrm{CH}_{2} \mathrm{Cl}_{2}$ $(0.38 \mathrm{~mL})$ was added methyl chloroformate $(86.4 \mu \mathrm{L}, 1.13 \mathrm{mmol})$ at $0{ }^{\circ} \mathrm{C}$ under an Ar atmosphere. After being stirred at same temperature for $30 \mathrm{~min}$, the reaction mixture was concentrated. The residue was purified by flash chromatography over silica gel with $n$-hexane-EtOAc (1:1) to give the title compound $\mathbf{5 2 b}$ as a colorless solid (29.2 $\mathrm{mg}, 81 \%$ ): $\mathrm{mp} 157-158{ }^{\circ} \mathrm{C}$ (from $n$-hexane); IR (neat) $\mathrm{cm}^{-1}: 1699$ $(\mathrm{C}=\mathrm{O}), 1577(\mathrm{C}=\mathrm{N}) ;{ }^{1} \mathrm{H}-\mathrm{NMR}\left(400 \mathrm{MHz}, \mathrm{CDCl}_{3}\right) \delta: 1.37\left(\mathrm{~s}, 9 \mathrm{H}, 3 \times \mathrm{CH}_{3}\right), 1.46$ $\left(\mathrm{t}, J=5.6 \mathrm{~Hz}, 4 \mathrm{H}, 2 \times \mathrm{CH}_{2}\right), 3.44\left(\right.$ br s, $\left.4 \mathrm{H}, 2 \times \mathrm{CH}_{2}\right), 3.56\left(\right.$ br s, $\left.2 \mathrm{H}, \mathrm{CH}_{2}\right), 3.70$ (s, 3H, $\left.\mathrm{CH}_{3}\right), 3.81\left(\mathrm{~s}, 2 \mathrm{H}, \mathrm{CH}_{2}\right), 7.29-7.33(\mathrm{~m}, 2 \mathrm{H}, \mathrm{Ar}), 8.05(\mathrm{~d}, J=8.5 \mathrm{~Hz}, 1 \mathrm{H}$, $\mathrm{Ar}) ;{ }^{13} \mathrm{C}-\mathrm{NMR}\left(100 \mathrm{MHz}, \mathrm{CDCl}_{3}\right) \delta: 29.9$ (3C), 30.1, 32.2 (2C), 39.9 (2C), 50.8, 52.5, 54.3, 55.2, 124.7, 126.1, 126.8, 129.3, 130.0, 130.9, 137.7, 146.3, 155.9; HRMS (FAB): $m / z$ calcd for $\mathrm{C}_{21} \mathrm{H}_{28} \mathrm{BrN}_{4} \mathrm{O}_{2} \mathrm{~S}[\mathrm{M}+\mathrm{H}]^{+}$479.1116; found: 479.1115 .

Compound 53b. Using the general procedure as described for 25a, compound 52b $(6.4 \mathrm{mg}, 0.013 \mathrm{mmol})$ was allowed to react for $2 \mathrm{~h}$ with TFA $(1.0 \mathrm{~mL})$ and MS4A (150 mg). Purification by flash chromatography over aluminum oxide with n-hexane-EtOAc (9:3) gave the title compound 53b as colorless solid (4.0 $\mathrm{mg}$, $73 \%$ ): mp 139-141 ${ }^{\circ} \mathrm{C}$ (from MeCN-H $\mathrm{H}_{2} \mathrm{O}$ ); IR (neat) $\mathrm{cm}^{-1}: 1692(\mathrm{C}=\mathrm{O}), 1626$ $(\mathrm{C}=\mathrm{N}), 1573(\mathrm{C}=\mathrm{N}) ;{ }^{1} \mathrm{H}-\mathrm{NMR}\left(400 \mathrm{MHz}, \mathrm{CDCl}_{3}\right) \delta: 1.49(\mathrm{t}, J=5.7 \mathrm{~Hz}, 4 \mathrm{H}$, $\left.2 \times \mathrm{CH}_{2}\right), 3.45-3.57\left(\mathrm{~m}, 6 \mathrm{H}, 3 \times \mathrm{CH}_{2}\right), 3.70\left(\mathrm{~s}, 3 \mathrm{H}, \mathrm{CH}_{3}\right), 3.91\left(\mathrm{~s}, 2 \mathrm{H}, \mathrm{CH}_{2}\right), 7.22$ $(\mathrm{d}, J=2.0 \mathrm{~Hz}, 1 \mathrm{H}, \mathrm{Ar}), 7.31$ (br s, $1 \mathrm{H}, \mathrm{NH}), 7.34$ (dd, $J=8.8,2.0 \mathrm{~Hz}, 1 \mathrm{H}, \mathrm{Ar})$, $8.10(\mathrm{~d}, J=8.8 \mathrm{~Hz}, 1 \mathrm{H}, \mathrm{Ar}) ;{ }^{13} \mathrm{C}-\mathrm{NMR}\left(100 \mathrm{MHz}, \mathrm{CDCl}_{3}\right) \delta: 29.5,32.2(2 \mathrm{C})$, 39.7 (2C), 50.0, 52.6, 54.6, 125.0, 125.3, 126.0, 129.6, 130.5, 130.6, 145.1, 152.6, 155.9; HRMS (FAB): $m / z$ calcd for $\mathrm{C}_{17} \mathrm{H}_{20} \mathrm{BrN}_{4} \mathrm{O}_{2} \mathrm{~S}[\mathrm{M}+\mathrm{H}]^{+} 423.0490$; found: 423.0492 .

\subsubsection{Synthesis of 1'-Acetyl-9-bromo-2H- spiro(benzo[e]pyrimido[1,2-c][1,3]thiazine-3,4'- piperidin)-6(4H)-imine (53c)}

\footnotetext{
$1^{\prime}$-Acetyl-9-bromo- $N$-(tert-butyl)- $2 H$-spiro(benzo[ $\left.e\right]$ pyrimido[1,2-c $][1,3]$ thiazine-3,4 $\mathbf{4}^{\prime}$-piperidin)-6(4H)-imine (52c). Using the general procedure as described for 52b, 9-bromo-N-(tert-butyl)-1'-(4-methoxybenzyl)-2H-spiro(benzo[e]pyrimido[1,2-c][1,3]thiazine-3,4'-piperidin)-6(4H)-imine 52a (40.6 mg, $0.075 \mathrm{mmol})$
} 
was allowed to react for $10 \mathrm{~min}$ with $\mathrm{AcCl}(53.3 \mu \mathrm{L}, 0.75 \mathrm{mmol})$. Purification by flash chromatography over aluminum oxide with n-hexane-EtOAc (1:1) gave the title compound 52c as colorless solid (33.3 mg, $96 \%$ ): $\mathrm{mp} 181-182{ }^{\circ} \mathrm{C}$ (from $\mathrm{CHCl}_{3}-\mathrm{n}$-hexane); IR (neat) $\mathrm{cm}^{-1}: 1632(\mathrm{C}=\mathrm{O}), 1578(\mathrm{C}=\mathrm{N}) ;{ }^{1} \mathrm{H}-\mathrm{NMR}$ $\left(400 \mathrm{MHz}, \mathrm{CDCl}_{3}\right) \delta: 1.37\left(\mathrm{~s}, 9 \mathrm{H}, 3 \times \mathrm{CH}_{3}\right), 1.46-1.52\left(\mathrm{~m}, 4 \mathrm{H}, 2 \times \mathrm{CH}_{2}\right), 2.10$ $\left(\mathrm{s}, 3 \mathrm{H}, \mathrm{CH}_{3}\right), 3.45-3.56(\mathrm{~m}, 5 \mathrm{H}, 5 \times \mathrm{CH}), 3.72-3.78(\mathrm{~m}, 2 \mathrm{H}, 2 \times \mathrm{CH}), 3.90(\mathrm{~d}$, $\mathrm{J}=13.4 \mathrm{~Hz}, 1 \mathrm{H}, \mathrm{CH}), 7.29-7.33(\mathrm{~m}, 2 \mathrm{H}), 8.05(\mathrm{~d}, \mathrm{~J}=8.5 \mathrm{~Hz}, 1 \mathrm{H}) ;{ }^{13} \mathrm{C}-\mathrm{NMR}$ $\left(100 \mathrm{MHz} \mathrm{CDCl}_{3}\right) \delta: 21.4,29.9$ (3C), 30.3, 32.2, 32.8, 37.5, 42.6, 50.8, 54.4, 55.1, $124.7,126.0,126.9,129.3,130.0,130.8,137.7,146.3,168.8 ;$ HRMS (FAB): m/z calcd for $\mathrm{C}_{21} \mathrm{H}_{28} \mathrm{BrN}_{4} \mathrm{OS}[\mathrm{M}+\mathrm{H}]^{+}$463.1167; found: 463.1164 .

Compound 53c. Using the general procedure as described for $\mathbf{2 5 a}$, compound 52c $(6.5 \mathrm{mg}, 0.014 \mathrm{mmol})$ was allowed to react for $2 \mathrm{~h}$ with TFA $(1.0 \mathrm{~mL})$ and MS4 $\AA$ (150 mg). Purification by flash chromatography over aluminum oxide with n-hexane-EtOAc (1:2) gave the title compound 53c as colorless solid (4.5 $\mathrm{mg}$, $79 \%$ ): $\mathrm{mp}$ 147-148 ${ }^{\circ} \mathrm{C}$ (from $\mathrm{CHCl}_{3}-\mathrm{n}$-hexane); IR (neat) $\mathrm{cm}^{-1}: 1625(\mathrm{C}=\mathrm{O})$, $1573(\mathrm{C}=\mathrm{N}) ;{ }^{1} \mathrm{H}-\mathrm{NMR}\left(400 \mathrm{MHz}, \mathrm{CDCl}_{3}\right) \delta: 1.49-1.55\left(\mathrm{~m}, 4 \mathrm{H}, 2 \times \mathrm{CH}_{2}\right), 2.09$ $\left(\mathrm{s}, 3 \mathrm{H}, \mathrm{CH}_{3}\right), 3.47-3.61(\mathrm{~m}, 5 \mathrm{H}, 5 \times \mathrm{CH}), 3.70-3.77(\mathrm{~m}, 1 \mathrm{H}, \mathrm{CH}), 3.84(\mathrm{~d}$, $\mathrm{J}=13.4 \mathrm{~Hz}, 1 \mathrm{H}, \mathrm{CH}), 4.04(\mathrm{~d}, \mathrm{~J}=13.2 \mathrm{~Hz}, 1 \mathrm{H}, \mathrm{CH}), 7.22(\mathrm{~d}, \mathrm{~J}=1.2 \mathrm{~Hz}, 1 \mathrm{H}$, Ar), $7.33-7.36(\mathrm{~m}, 2 \mathrm{H}, \mathrm{Ar}, \mathrm{NH}), 8.10(\mathrm{~d}, \mathrm{~J}=8.8 \mathrm{~Hz}, 1 \mathrm{H}, \mathrm{Ar}) ;{ }^{13} \mathrm{C}-\mathrm{NMR}$ $\left(100 \mathrm{MHz}, \mathrm{CDCl}_{3}\right) \delta: 21.4,29.7,32.1,32.7,37.3,42.4,49.8,54.7,124.9,125.3$, 126.0, 129.6, 130.4, 130.6, 145.1, 152.6, 168.9; Anal. calcd for $\mathrm{C}_{17} \mathrm{H}_{19} \mathrm{BrN}_{4} \mathrm{OS}$ : C, 50.13; H, 4.70; N, 13.75. Found: C, 50.24; H, 4.78; N, 13.57.

\title{
3.1.87 Synthesis of 9-Bromo-1'-(methanesulfonyl)-2H- spiro(benzo[e]pyrimido[1,2-c][1,3]thiazine-3,4'- piperidin)-6(4H)-imine (53d)
}

\begin{abstract}
9-Bromo- $N$-(tert-butyl)-1' -(methanesulfonyl)- $2 H$-spiro(benzo[e]pyrimido[1,2c] $[1,3]$ thiazine-3,4' -piperidin)-6(4H)-imine (52d). To the solution of 9-bromo- $\mathrm{N}$ (tert-butyl)-1'-(4-methoxybenzyl)-2H-spiro(benzo[e]pyrimido[1,2-c][1,3]thiazine$3,4^{\prime}$-piperidin)-6(4H)-imine 52a $(54.2 \mathrm{mg}, 0.10 \mathrm{mmol})$ in $\mathrm{CH}_{2} \mathrm{Cl}_{2}(0.5 \mathrm{~mL})$ were added $\mathrm{Et}_{3} \mathrm{~N}(28.9 \mu \mathrm{L}, 0.20 \mathrm{mmol})$ and 1-chloroethyl chloroformate $(21.8 \mu \mathrm{L}$, $0.20 \mathrm{mmol}$ ) at $0{ }^{\circ} \mathrm{C}$ under an $\mathrm{Ar}$ atmosphere. After being stirred at same temperature for $30 \mathrm{~min}$, the reaction mixture was concentrated. The residue was dissolved in $\mathrm{MeOH}(2.0 \mathrm{~mL})$. After being stirred under reflux for $10 \mathrm{~min}$, the reaction mixture was concentrated. The residue was dissolved in $\mathrm{CHCl}_{3}$, and was washed with sat. $\mathrm{NaHCO}_{3}$, brine, and dried over $\mathrm{MgSO}_{4}$. After concentration, the residue was dissolved in $\mathrm{CH}_{2} \mathrm{Cl}_{2}(1.0 \mathrm{~mL})$ and $\mathrm{Et}_{3} \mathrm{~N}(28.9 \mu \mathrm{L}, 0.20 \mathrm{mmol})$, and $\mathrm{MsCl}(15.5 \mu \mathrm{L}, 0.20 \mathrm{mmol})$ was added at $\mathrm{rt}$ under an $\mathrm{Ar}$ atmosphere. After being stirred at $\mathrm{rt}$ for $10 \mathrm{~min}$, the reaction mixture was washed with sat. $\mathrm{NaHCO}_{3}$, brine, and dried over $\mathrm{MgSO}_{4}$. After concentration, the residue was purified by flash chromatography over aluminum oxide with n-hexane-EtOAc (6:4) to give the title
\end{abstract}


compound 52d as a colorless solid (40.9 mg, $82 \%$ ): mp $177{ }^{\circ} \mathrm{C}$ (from $\mathrm{CHCl}_{3}-\mathrm{n}$ hexane); IR (neat) $\mathrm{cm}^{-1}$ : $1577(\mathrm{C}=\mathrm{N}), 1331\left(\mathrm{NSO}_{2}\right), 1155\left(\mathrm{NSO}_{2}\right) ;{ }^{1} \mathrm{H}-\mathrm{NMR}$ $\left(400 \mathrm{MHz}, \mathrm{CDCl}_{3}\right) \delta: 1.38\left(\mathrm{~s}, 9 \mathrm{H}, 3 \times \mathrm{CH}_{3}\right), 1.62\left(\mathrm{t}, \mathrm{J}=5.5 \mathrm{~Hz}, 4 \mathrm{H}, 2 \times \mathrm{CH}_{2}\right)$, 2.80 (s, 3H, $\left.\mathrm{CH}_{3}\right), 3.21-3.27\left(\mathrm{~m}, 2 \mathrm{H}, \mathrm{CH}_{2}\right), 3.31-3.37\left(\mathrm{~m}, 2 \mathrm{H}, \mathrm{CH}_{2}\right), 3.46(\mathrm{~s}, 2 \mathrm{H}$, $\mathrm{CH}_{2}$ ), 3.84 (s, 2H, $\left.\mathrm{CH}_{2}\right), 7.29-7.33$ (m, 2H, Ar), 8.05 (d, J = 8.5 Hz, $\left.1 \mathrm{H}, \mathrm{Ar}\right) ;{ }^{13} \mathrm{C}-$ NMR (100 MHz, $\mathrm{CDCl}_{3}$ ) $\delta: 29.8,29.9$ (3C), 32.0 (2C), 34.7, 42.0 (2C), 50.1, 54.4, 55.1, 124.8, 125.9, 126.9, 129.4, 130.0, 130.8, 137.9, 146.3; HRMS (FAB): m/z calcd for $\mathrm{C}_{20} \mathrm{H}_{28} \mathrm{BrN}_{4} \mathrm{O}_{2} \mathrm{~S}_{2}[\mathrm{M}+\mathrm{H}]^{+}$499.0837; found: 499.0840 .

Compound 53d. Using the general procedure as described for 25a, compound 52d $(9.2 \mathrm{mg}, 0.018 \mathrm{mmol})$ was allowed to react for $2 \mathrm{~h}$ with TFA $(1.0 \mathrm{~mL})$ and MS4A (150 mg). Purification by flash chromatography over aluminum oxide with n-hexane-EtOAc (1:1) gave the title compound 53d as colorless solid $(5.3 \mathrm{mg}$, $65 \%$ ): mp 171-172 ${ }^{\circ} \mathrm{C}$ (from $\mathrm{CHCl}_{3}-\mathrm{n}$-hexane); IR (neat) $\mathrm{cm}^{-1}: 1621(\mathrm{C}=\mathrm{N}$ ), $1564(\mathrm{C}=\mathrm{N}), 1320\left(\mathrm{NSO}_{2}\right), 1152\left(\mathrm{NSO}_{2}\right) ;{ }^{1} \mathrm{H}-\mathrm{NMR}\left(400 \mathrm{MHz}, \mathrm{CDCl}_{3}-\mathrm{CD}_{3} \mathrm{OD}\right) \delta$ : 1.64-1.67 (m, 4H, $\left.2 \times \mathrm{CH}_{2}\right), 2.82\left(\mathrm{~s}, 3 \mathrm{H}, \mathrm{CH}_{3}\right), 3.19-3.25\left(\mathrm{~m}, 2 \mathrm{H}, \mathrm{CH}_{2}\right)$, 3.35-3.41 (m, 2H, CH$), 3.52\left(\mathrm{~s}, 2 \mathrm{H}, \mathrm{CH}_{2}\right), 3.92\left(\mathrm{~s}, 2 \mathrm{H}, \mathrm{CH}_{2}\right), 7.24(\mathrm{~d}, \mathrm{~J}=2.0 \mathrm{~Hz}$, $1 \mathrm{H}, \mathrm{Ar}), 7.37(\mathrm{dd}, \mathrm{J}=8.5,2.0 \mathrm{~Hz}, 1 \mathrm{H}, \mathrm{Ar}), 8.07(\mathrm{~d}, \mathrm{~J}=8.5 \mathrm{~Hz}, 1 \mathrm{H}, \mathrm{Ar}) ;{ }^{13} \mathrm{C}-$ NMR (100 MHz, $\left.\mathrm{CDCl}_{3}-\mathrm{CD}_{3} \mathrm{OD}\right) \delta: 29.0,31.9$ (2C), 34.7, 41.7 (2C), 49.2, 54.6, 124.7, 125.4, 126.0, 129.7, 130.3, 130.4, 145.3, 153.1; HRMS (FAB): m/z calcd for $\mathrm{C}_{16} \mathrm{H}_{20} \mathrm{BrN}_{4} \mathrm{O}_{2} \mathrm{~S}_{2}[\mathrm{M}+\mathrm{H}]^{+} 443.0211$; found: 443.0210 .

\subsubsection{Synthesis of $1^{\prime}$-(Aminocarbonyl)-9-bromo-2H- spiro(benzo[e]pyrimido[1,2-c][1,3]thiazine-3,4'- piperidin)-6(4H)-imine (53e)}

\section{$1^{\prime}$-(Aminocarbonyl)-9-bromo- $N$-(tert-butyl)-2H-spiro(benzo[e]pyrimido[1,2-c]} $[1,3]$ thiazine-3,4'-piperidin)-6(4H)-imine (52e). Using the general procedure as described for 52d, 9-bromo-N-(tert-butyl)-1'-(4-methoxybenzyl)-2H-spiro (benzo [e]pyrimido[1,2-c][1,3]thiazine-3, $4^{\prime}$-piperidin)-6(4H)-imine $\quad$ 52a $\quad(54.2 \mathrm{mg}$, $0.10 \mathrm{mmol})$ was allowed to react with 1 -chloroethyl chloroformate $(21.8 \mu \mathrm{L}$, $0.20 \mathrm{mmol}$ ) followed with $\mathrm{N}$-trimethylsilylisocyanate $(26.5 \mu \mathrm{L}, 0.20 \mathrm{mmol})$. Purification by flash chromatography over aluminum oxide with EtOAc-MeOH (1:0 to 9:1) gave the title compound 52e as colorless solid (11.8 $\mathrm{mg}, 29 \%): \mathrm{mp}$ 203-205 ${ }^{\circ} \mathrm{C}$ (from $\mathrm{CHCl}_{3}-\mathrm{n}$-hexane); IR (neat) $\mathrm{cm}^{-1}$ : $1649(\mathrm{C}=\mathrm{O}), 1577(\mathrm{C}=\mathrm{N})$; ${ }^{1} \mathrm{H}-\mathrm{NMR}\left(400 \mathrm{MHz}, \mathrm{CDCl}_{3}\right) \delta: 1.37\left(\mathrm{~s}, 9 \mathrm{H}, 3 \times \mathrm{CH}_{3}\right), 1.51(\mathrm{t}, \mathrm{J}=5.6 \mathrm{~Hz}, 4 \mathrm{H}$, $\left.2 \times \mathrm{CH}_{2}\right), 3.37-3.51\left(\mathrm{~m}, 6 \mathrm{H}, 3 \times \mathrm{CH}_{2}\right), 3.82\left(\mathrm{~s}, 2 \mathrm{H}, \mathrm{CH}_{2}\right), 4.46\left(\mathrm{~s}, 2 \mathrm{H}, \mathrm{NH}_{2}\right)$, 7.30-7.33 (m, 2H, Ar), 8.05 (d, J = 8.5 Hz, 1H, Ar); ${ }^{13} \mathrm{C}-\mathrm{NMR}(100 \mathrm{MHz}$, $\mathrm{CDCl}_{3}$ ) $\delta: 29.9$ (3C), 30.0, 32.1 (2C), 40.3 (2C), 50.9, 54.4, 55.1, 124.7, 126.0, 126.8, 129.3, 130.0, 130.9, 137.6, 146.3, 158.0; HRMS (FAB): m/z calcd for $\mathrm{C}_{20} \mathrm{H}_{27} \mathrm{BrN}_{5} \mathrm{OS}[\mathrm{M}+\mathrm{H}]^{+}$464.1120; found: 464.1122.

Compound 53e. Using the general procedure as described for $\mathbf{2 5 a}$, compound 52e $(5.1 \mathrm{mg}, 0.011 \mathrm{mmol})$ was allowed to react for $2 \mathrm{~h}$ with TFA $(1.0 \mathrm{~mL})$ and 
MS4Å (150 mg). Purification by flash chromatography over aluminum oxide with EtOAc-MeOH (1:0 to 9:1) gave the title compound 53e as colorless solid (4.2 $\mathrm{mg}$, $94 \%$ ): $\mathrm{mp} 222{ }^{\circ} \mathrm{C}$ (from $\mathrm{MeOH}-\mathrm{CHCl}_{3}-\mathrm{n}$-hexane); IR (neat) $\mathrm{cm}^{-1}: 1651(\mathrm{C}=\mathrm{O})$, $1624(\mathrm{C}=\mathrm{N}), 1585(\mathrm{C}=\mathrm{N}) ;{ }^{1} \mathrm{H}-\mathrm{NMR}\left(400 \mathrm{MHz}, \mathrm{DMSO}_{-} \mathrm{d}_{6}\right) \delta: 1.31(\mathrm{t}, \mathrm{J}=5.6 \mathrm{~Hz}$, $\left.4 \mathrm{H}, 2 \times \mathrm{CH}_{2}\right), 3.20-3.38\left(\mathrm{~m}, 4 \mathrm{H}, 2 \times \mathrm{CH}_{2}\right), 3.45\left(\mathrm{~s}, 2 \mathrm{H}, \mathrm{CH}_{2}\right), 3.84\left(\mathrm{~s}, 2 \mathrm{H}, \mathrm{CH}_{2}\right)$, $5.88\left(\mathrm{~s}, 2 \mathrm{H}, \mathrm{NH}_{2}\right), 7.42(\mathrm{dd}, \mathrm{J}=8.5,1.5 \mathrm{~Hz}, 1 \mathrm{H}), 7.59(\mathrm{~d}, \mathrm{~J}=1.5 \mathrm{~Hz}, 1 \mathrm{H}), 8.08$ $(\mathrm{d}, \mathrm{J}=8.5 \mathrm{~Hz}, 1 \mathrm{H}, \mathrm{Ar}), 8.91(\mathrm{~s}, 1 \mathrm{H}, \mathrm{NH}) .{ }^{13} \mathrm{C}-\mathrm{NMR}\left(125 \mathrm{MHz}, \mathrm{DMSO}_{-}\right) \delta$ : 29.0, 31.6 (2C), $39.5 \pm 1.0$ (2C), 48.9, 54.0, 124.3, 124.7, 126.0, 129.0, 130.2, 131.2, 144.0, 149.2, 158.0; HRMS (FAB): $\mathrm{m} / \mathrm{z}$ calcd for $\mathrm{C}_{16} \mathrm{H}_{19} \mathrm{BrN}_{5} \mathrm{OS}$ $[\mathrm{M}+\mathrm{H}]^{+}$408.0494; found: 408.0496.

\subsubsection{Determination of Anti-HIV Activity}

The sensitivity of HIV-1 $1_{\text {IIIB }}$ strain was determined by the MAGI assay. The target cells (HeLa-CD4/CCR5-LTR/ $\beta$-gal; $10^{4}$ cells/well) were plated in 96-well flat microtiter culture plates. On the following day, the cells were inoculated with the HIV-1 (60 MAGI U/well, giving 60 blue cells after $48 \mathrm{~h}$ of incubation) and cultured in the presence of various concentrations of the test compounds in fresh medium. Forty-eight hours after viral exposure, all the blue cells stained with $\mathrm{X}$-Gal (5-bromo-4-chloro-3-indolyl- $\beta$-D-galactopyranoside) were counted in each well. The activity of test compounds was determined as the concentration that blocked HIV-1 infection by $50 \%$ (50 \% effective concentration $\left.\left[\mathrm{EC}_{50}\right]\right) . \mathrm{EC}_{50}$ was determined by using the following formula:

$$
\mathrm{EC}_{50}=10^{\wedge}[\log (\mathrm{A} / \mathrm{B}) \times(50-\mathrm{C}) /(\mathrm{D}-\mathrm{C})+\log (\mathrm{B})],
$$

wherein

A: of the two points on the graph which bracket $50 \%$ inhibition, the higher concentration of the test compound,

B: of the two points on the graph which bracket $50 \%$ inhibition, the lower concentration of the test compound,

$\mathrm{C}$ : inhibitory activity (\%) at the concentration B,

D: inhibitory activity (\%) at the concentration A.

\section{References}

1. Chockalingam, K., Simeon, R.L., Rice, C.M., Chen, Z.: Proc. Natl. Acad. Sci. U.S.A. 107, 3764-3769 (2010)

2. Chamoun, A.M., Chockalingam, K., Bobardt, M., Simeon, R., Chang, J., Gallay, P., Chen, Z.: Antimicrob. Agents Chemother. 56, 672-681 (2012)

3. Ishihara, M., Togo, H.: Tetrahedron 63, 1474-1480 (2007) 
4. Wolfe, J.P., Wagaw, S., Marcoux, J.-F., Buchwald, S.L.: Acc. Chem. Res. 31, 805-818 (1998)

5. Hartwig, J.P.: Angew. Chem. Int. Ed. 37, 2046-2065 (1998)

6. Kunz, K., Scholz, U., Ganzer, D.: Synlett. 2428-2439 (2003)

7. Arvela, R.K., Pasquini, S., Larhed, M.J.: Org. Chem. 72, 6390-6396 (2007)

8. Miyaura, N., Suzuki, A.: Chem. Rev. 95, 2457-2483 (1995)

9. Mitschb, A., Altenkämpera, M., Sattlerc, I., Schlitzer, M.: Arch. Pharm. Chem. Life Sci. 338, 9-17 (2005)

10. Watanabe, K., Negi, S., Sugiura, Y., Kiriyama, A., Honbo, A., Iga, K., Kodama, E.N., Naitoh, T., Matsuoka, M., Kano, K.: Chem. Asian J. 5, 825-834 (2010)

11. Drake, R.R., Neamati, N., Hong, H., Pilon, A.A., Sunthankar, P., Hume, S.D., Milne, G.W.A., Pommier, Y.: Proc. Natl. Acad. Sci. U.S.A. 95, 4170-4175 (1998)

12. Lin, W., Li, K., Doughty, M.B.: Bioorg. Med. Chem. 10, 4131-4141 (2002)

13. Al-Mawsawi, L.Q., Fikkert, V., Dayam, R., Witvrouw, M., Burke Jr, T.R., Borchers, C.H., Neamati, N.: Proc. Natl. Acad. Sci. U.S.A. 103, 10080-10085 (2006)

14. Baba, M., Scgols, D., Pauwels, R., Nakashima, H., De Clercq, E.J.: Acquir. Immune. Defic. Syndr. 3, 493-499 (1990)

15. Kilby, J.M., Eron, J.J.N.: Engl. J. Med. 348, 2228-2238 (2003)

16. Lalezari, J.P., Henry, K., O'Hearn, M., Montaner, J.S., Piliero, P.J., Trottier, B., Walmsley, S., Cohen, C., Kuritzkes, D.R., Eron Jr, J.J., Chung, J., DeMasi, R., Donatacci, L., Drobnes, C., Delehanty, J., Salgo, M.N.: Engl. J. Med. 348, 2175-2185 (2003)

17. Matthews, T., Salgo, M., Greenberg, M., Chung, J., DeMasi, R., Bolognesi, D.: Nat. Rev. Drug Discov. 3, 215-225 (2004)

18. Fischl, M.A., Richman, D.D., Grieco, M.H.N.: Engl. J. Med. 317, 185-191 (1987)

19. Merluzzi, V.J., Hargrave, K.D., Labadia, M., Grozinger, K., Skoog, M., Wu, J.C., Shih, C.-K., Eckner, K., Hattox, S., Adams, J., Rosehthal, A.S., Faanes, R., Eckner, R.J., Koup, R.A., Sullivan, J.L.: Science 250, 1411-1413 (1990)

20. Steigbigel, R.T., Cooper, D.A., Kumar, P.N., Eron, J.E., Schechter, M., Markowitz, M., Loutfy, M.R., Lennox, J.L., Gatell, J.M., Rockstroh, J.K., Katlama, C., Yeni, P., Lazzarin, A., Clotet, B., Zhao, J., Chen, J., Ryan, D.M., Rhodes, R.R., Killar, J.A., Gilde, L.R., Strohmaier, K.M., Meibohm, A.R., Miller, M.D., Hazuda, D.J., Nessly, M.L., DiNubile, M.J., Isaacs, R.D., Nguyen, B.-Y., Teppler, H.N.: Engl. J Med. 359, 339-354 (2008)

21. Witvrouw, M., Pannecouque, C., Switzer, W.M., Folks, T.M., De Clercq, E., Heneine, W.: Antivir. Ther. 9, 57-65 (2004) 